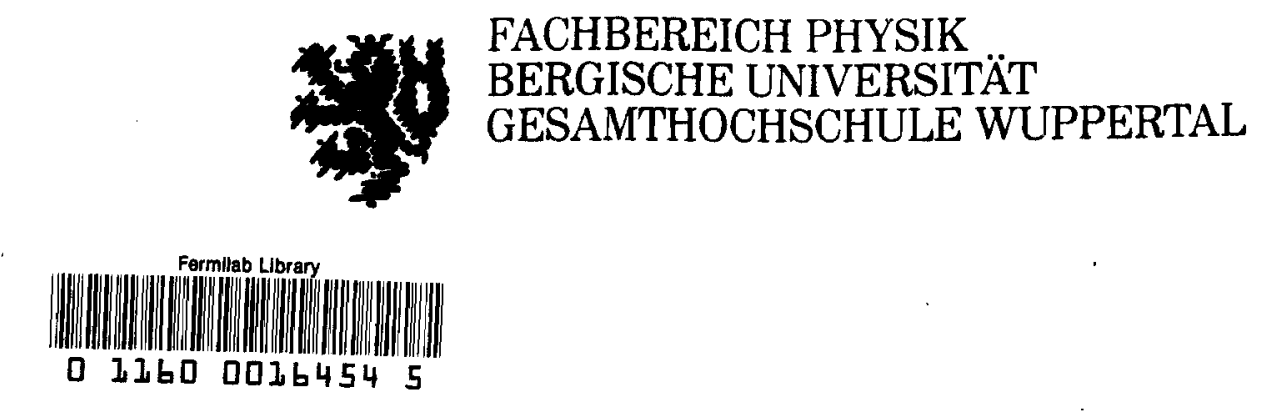

\title{
Longitudinale und transversale Impulsverteilungen der Hadronen im Endzustand der tiefinelastischen Myon-Nukleon-Streuung
}

\author{
Uwe Ecker
}

\author{
Fachbereich Physik \\ Bergische Universität Gesamthochschule Wuppertal \\ Bundesrepublik Deutschland
}





\title{
Longitudinale und transversale Impulsverteilungen der Hadronen im Endzustand der tiefinelastischen Myon-Nukleon-Streuung
}

\author{
Uwe Ecker
}

Fachbereich Physik

Bergische Universität Gesamthochschule Wuppertal Bundesrepublik Deutschland 


\section{Dissertation zur Erlangung des Doktorgrades}

des Fachbereiches Physik

der Bergischen Universität Gesamthochschule Wuppertal

vorgelegt von

Uwe Ecker

aus

Kirburg 


\section{Abstract}

The Fermilab experiment E665 took its first data during the period 1987/1988. 490 $\mathrm{GeV}$ and $100 \mathrm{GeV}$ muons are scattered off various nuclear targets. In this analysis a study of the longitudinal and transverse momentum distributions of charged final state hadrons produced in muon-deuterium-interactions is performed. Semi inclusive muoproduction data are presented in a kinematic regime up to a previously unreached invariant mass of $\sim 30 \mathrm{GeV}$. The data are discussed in the framework of the quark parton model and quantum chromodynamics. A comparison to various leptoproduction and $\mathrm{e}^{+} \mathrm{e}^{-}$-annihilation experiments is shown. Subsequently the data are confronted with predictions from the Lund model.

The data agree well with a QCD based parton model picture. 


\section{Inhaltsverzeichnis}

$\begin{array}{ll}\text { 1. Einleitung } & 1\end{array}$

2. Die tiefinelastische Myon-Nukleon-Streuung 3

2.1 Der Wirkungsquerschnitt $\ldots \ldots \ldots \ldots \ldots \ldots \ldots$

2.2 Das Parton-Modell . . . . . . . . . . . . . . 5

2.3 Das erweiterte Quark-Parton-Modell . . . . . . . . . . . . . 8

2.4 Die Quarkfragmentierung im Quark-Parton-Modell . . . . . . . . 10

2.5 Phänomenologische Monte-Carlo-Modelle zur Fragmentierung . . . 11

3. Das Experiment 665 am Fermilab 16

3.1 Das Tevatron . . . . . . . . . . . . . . . . 16

3.2 Die E665-Kollaboration $\ldots \ldots \ldots \ldots \ldots \ldots$

3.3 Die Strahlführung des Experimentes $665 \ldots \ldots \ldots \ldots$

3.4 Der Detektor des Experimentes $665 \ldots \ldots \ldots 22$

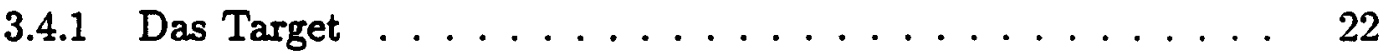

3.4.2 Die Spurnachweissysteme $\ldots \ldots \ldots \ldots \ldots$

3.4.3 Die E665-Detektoren zur Teilchenidentifikation und das elektromagnetische Kalorimeter . . . . . . . . . . 25

3.4 .4 Der Trigger. . . . . . . . . . . . . . . . 34

3.5 Datennahme und Überwachung . . . . . . . . . . . . 35

4. Die Datenaufbereitung und Rekonstruktion 39

4.1 Die Grundidee der E665-Rekonstruktionssoftware . . . . . . . . . . 39 
4.2 Der Datenfluß und die Rekonstruktionsprogramme . . . . . . . . 40

4.2.1 Alignment und Kalibration .............. 42

4.2.2 Das Erkennen von Teilchentrajektorien . . . . . . . . . 43

4.2.3 Die Spuranpassung .................... 44

4.2.4 Die Identifikation der Myonspuren . . . . . . . . . . 45

4.2.5 Die Vertex-Rekonstruktion .............. 46

4.2.6 Das Teilchenidentifikationsprogramm . . . . . . . . . 46

4.3 Das E665-Detektorsimulationsprogramm . . . . . . . . . 46

5. Die Datenanalyse $\quad 50$

5.1 Unkorrigierte Verteilungen .................. 50

5.2 Die Auswahl der Ereignisse . . . . . . . . . . . . . 52

5.3 Die Auswahl der primären hadronischen Spuren . . . . . . . . . 58

5.4 Korrekturen und systematische Fehler . . . . . . . . . . . 62

5.4.1 Die Akzeptanzkorrektur ............... 62

5.4.2 Strahlungskorrekturen................ 65

5.4.3 Systematische Fehler ................. 69

$\begin{array}{ll}\text { 6. Präsentation der Ergebnisse und Diskussion } & \mathbf{7 3}\end{array}$

6.1 Skalierte Energieverteilungen der Hadronen . . . . . . . . . 73

6.2 Die Verteilungen des Transversalimpulses . . . . . . . . . . . 84

6.3 Vergleich der Daten mit Modellvorhersagen ............ 94 
$\begin{array}{lr}\text { 7. Zusammenfassung } & 99\end{array}$

$\begin{array}{ll}\text { Danksagung } & 101\end{array}$

A Tabellierte Daten der differentiellen Multiplizitäten und Verteilungen des mittleren Transversalimpulses 102

$\begin{array}{ll}\text { Abbildungsverzeichnis } & 110\end{array}$

$\begin{array}{ll}\text { Tabellenverzeichnis } & 114\end{array}$

$\begin{array}{ll}\text { Literaturverzeichnis } & 116\end{array}$ 


\section{Einleitung}

Die Frage nach dem Aufbau der Materie und dem Zusammenwirken der Naturkräfte beschäftigt die Menschen schon seitdem uns schriftliche Überlieferungen erhalten sind. Die Griechen prägten den Begriff des Atoms, des unteilbaren Elementarbausteins.

Diese Frage hat über die Jahrhunderte nichts an Aktualität verloren. Der philosophische Ansatz wurde im Mittelalter durch den experimentellen Ansatz ergänzt, Hypothesen wurden durch Experimente nachprüfbar.

Eine stürmische Entwicklung zur Erforschung der Materie löste Rutherford durch seine Streuexperimente aus. Er beschoß dünne Goldfolien mit $\alpha$-Teilchen, maß die Verteilung der gestreuten Teilchen und leitete daraus eines der ersten Atommodelle ab [1].

Das Prinzip der Streuexperimente ist bis heute erfolgreich geblieben und hat wesentlich zum Verständnis der Struktur der Materie und der Wechselwirkungen zwischen den Elementarteilchen beigetragen. Der Aufbau der Materie läßt sich aus heutiger Sicht vereinfacht folgendermaßen skizzieren. Die Grundbausteine aller Materie sind Spin 1/2 tragende Fermionen: Quarks und Leptonen. Aus ihnen lassen sich alle bekannten Teilchen aufbauen. Die Einteilung dieser Fermionen kann in folgende Familien vorgenommen werden (z.B. [2]).

\begin{tabular}{|ll|ll|ll|c|}
\hline \multicolumn{5}{|c|}{ Familien } & Ladung \\
\hline \hline$\nu_{e}$ & Elektron-Neutrino & $\nu_{\mu}$ & Myon-Neutrino & $\nu_{\tau}$ & Tau-Neutrino & 0 \\
\hline e & Elektron & $\mu$ & Myon & $\tau$ & Tau & -1 \\
\hline $\mathbf{u}$ & Up-Quark & $\mathrm{c}$ & Charm-Quark & $\mathrm{t}^{1}$ & Top-Quark & $2 / 3$ \\
\hline $\mathrm{d}$ & Down-Quark & $\mathrm{s}$ & Strange-Quark & $\mathrm{b}$ & Bottom-Quark & $-1 / 3$ \\
\hline
\end{tabular}

Tabelle 1.1: Einteilung der fundamentalen Elementarteilchen nach [2]

Vier zwischen den Teilchen wirkende Kräfte sind bekannt: Die elektromagnetische, die schwache, die starke und die Gravitationswechselwirkung, die über den Austausch von Spin 1 (Photon, $Z^{0} W^{ \pm}$, Gluon entsprechend obiger Reihenfolge) bzw. Spin 2 (Graviton) tragenden Bosonen vermittelt werden. Die Quarks tragen die Eigenschaften aller Wechselwirkungsarten, die Leptonen hingegen keine 'Farbe', die Eigenschaft, die die Wechselwirkung über die starke Kraft ermöglicht. Die Neutrinos sind darüberhinaus elektrisch neutral und besitzen (falls überhaupt) eine verschwindend

\footnotetext{
${ }^{1}$ Die Existenz des Top-Quark ist zur Zeit noch nicht experimentell nachgewiesen
} 
kleine Masse, so daß sie im wesentlichen nur über die schwache Kraft wechselwirken. Hadronen sind aus Quarks aufgebaut, Leptonen elementar. Das Wasserstoffatom ist in diesem Bild aus uud-Quarks (Kern) und einem Elektron (Hülle) aufgebaut. Die Quarks werden durch die starke Wechselwirkung im Kern zusammengehalten, das Elektron durch die elektromagnetische Kraft im Atom gebunden.

Eines der vielen Streuexperimente, die zum tieferen Verständnis der Materie durchgeführt werden, ist das Experiment 665 [3] am FNAL2. Mit der zur Zeit höchsten Strahlenergie von $490 \mathrm{GeV}$ werden Myonen am Proton, Deuteron und Xenon gestreut.

Die erste E665-Datennahme erfolgte im Zeitraum Juni 1987 bis Februar 1988. In dieser Arbeit werden Daten dieser ersten Periode analysiert und semi-inklusive Verteilungen des hadronischen Endzustandes diskutiert.

Nach theoretischen Vorbemerkungen im Kapitel 2 wird das Experiment 665 am Fermilab in Kapitel 3 vorgestellt. Kapitel 4 und 5 beinhalten die Datenaufbereitung und Analyse. Im Kapitel 6 werden die Ergebnisse präsentiert und im Vergleich zu anderen Experimenten und Modellen diskutiert.

\footnotetext{
${ }^{2}$ Fermi National Accelerator Laboratory
} 


\section{Die tiefinelastische Myon-Nukleon-Streuung}

Zur Untersuchung der Kernstrukturen in Streuexperimenten eignen sich Myonen besonders. Erstens wechselwirken die strukturlosen Leptonen im wesentlichen ${ }^{1}$ nur über die elektromagnetische Wechselwirkung, so daß der Myon-Nukleon Vertex im Rahmen der Quantenelektrodynamik (QED) gut berechenbar ist. Zweitens sind Strahlungsverluste 2.B. gegenüber Elektronen durch einen Faktor $\left(m_{e} / m_{\mu}\right)^{2} \simeq 1 / 10^{4}$ untersetzt, und drittens lassen sich Myonen im Experiment aufgrund ihrer geringen Wechselwirkung mit Materie gut von anderen Teilchenarten unterscheiden.

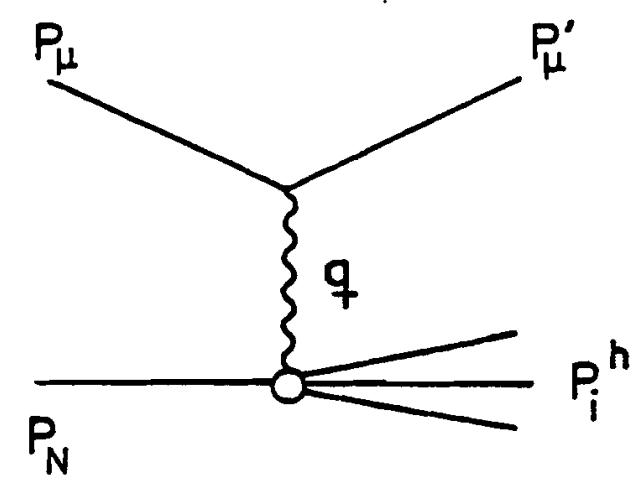

Abbildung 2.1: Ein-Photon-Austausch Feynman-Graph

Abbildung 2.1 stellt den entsprechenden Feynman-Graphen zum Ein-Photon-Austausch dar. $P_{\mu}$ und $P_{\mu}^{\prime}$ sind die Viererimpulse des ein- und auslaufenden Myons, $\mathrm{q}$ ist der Viererimpulsübertrag des virtuellen Photons, $P_{N}$ der Viererimpuls des Targetkerns, $P_{i}^{h}$ der des i-ten Hadron. Die gebräuchlichsten kinematischen Variablen sind in $\mathrm{Ta}$ belle 2.1 zusammengefaßt.

\subsection{Der Wirkungsquerschnitt}

Der doppelt differentielle Wirkungsquerschnitt für den Prozeß in Abbildung 2.1 läßt sich darstellen als [4],[5]:

$$
\frac{d^{2} \sigma}{d \Omega d E^{\prime}}=\frac{\alpha^{2}}{Q^{4}} \frac{E^{\prime}}{E}|A|^{2} \equiv \frac{\alpha^{2}}{Q^{4}} \frac{E^{\prime}}{E} L_{\mu \nu} W^{\mu \nu}
$$

\footnotetext{
${ }^{1}$ Tatsächlich tragen die Leptonen auch eine schwache Ladung, wie bereits in der Einleitung erwähnt, allerdings ist der Beitrag zum gesamten Wirkungsquerschnitt über den Propagatorterm $1 /\left(Q^{2}+M_{z}^{2}\right)^{2}$ erheblich unterdrückt, wobei $M_{z}$ die Masse des $2^{0} \cong 91 \mathrm{GeV}$ ist .
} 


\begin{tabular}{|ll|}
\hline & Myonvariablen \\
\hline$M=$ & Ruhemasse des Proton \\
$m_{\mu}=$ & Ruhemasse des Myon \\
$E_{0}=$ & Energie des einlaufenden Myons \\
$E^{\prime}=$ & Energie des auslaufenden Myons \\
$P_{\mu}=$ & $\left(E_{0}, \mathbf{P}\right)=$ Viererimpuls des einlaufenden Myons \\
$P_{\mu}^{\prime}=$ & $\left(E^{\prime}, \mathbf{P}^{\prime}\right)=$ Viererimpuls des auslaufenden Myons \\
$P_{N}=$ & $(M, 0)=$ Viererimpuls des Target-Protons \\
$\mathrm{q}=$ & $\left(P_{\mu}-P_{\mu}^{\prime}\right)=(\nu, \mathbf{q})$ \\
$\theta$ & $=$
\end{tabular}

Tabelle 2.1: Definition der kinematischen Variablen in der Myon-Nukleon-Streuung 
Wobei $L_{\mu \nu}$ den leptonischen Vertex beschreibt und der Tensor $W^{\mu \nu}$ den hadronischen Vertex kennzeichnet. $L_{\mu \nu}$ ist im Rahmen der QED exakt berechenbar. Berücksichtigung der Lorentzinvarianz, Paritätserhaltung und Stromerhaltung führt zum doppelt differentiellen Wirkungsquerschnitt der Form:

$$
\frac{d^{2} \sigma}{d \Omega d E^{\prime}}=\frac{4 \alpha^{2} E^{\prime 2}}{Q^{4}}\left\{\cos ^{2} \frac{\theta}{2} W_{2}\left(\nu, q^{2}\right)+2 W_{1}\left(\nu, q^{2}\right) \sin ^{2} \frac{\theta}{2}\right\}
$$

Hierbei bezeichnen $W_{1}\left(=\frac{1}{M} F_{1}\right)$ und $W_{2}\left(=\frac{1}{\nu} F_{2}\right)$ Strukturfunktionen, die den Kernaufbau beschreiben und experimentell bestimmt werden. Die Gleichung für den inelastischen Wirkungsquerschnitt schreibt sich in der gleichen Form wie die Rosenbluthformel für die elastische Streuung. Dort enthalten die Strukturfunktionen die Formfaktoren, deren Fouriertransformierte die Ladungsverteilung im Kern beschreibt. Eine Interpretation der Strukturfunktionen im inelastischen Fall wird weiter unten gegeben.

\subsection{Das Parton-Modell}

Für große $\nu$ und $q^{2}$ sagte Bjorken voraus [6], daß sich die Abhängigkeit der inelastischen Formfaktoren von den beiden unabhängigen Lorentzinvarianten auf die Abhängigkeit von nur einer Variablen $x\left(=-q^{2} / M \nu\right)$ reduziert.

$$
\begin{gathered}
\lim _{q^{2} \rightarrow \infty, \nu / q^{2} f e s t} \nu W_{2}\left(q^{2}, \nu\right)=M F_{2}\left(-q^{2} / M \nu\right) \\
\lim _{q^{2} \rightarrow \infty, \nu / q^{2} \text { fest }} M W_{1}\left(q^{2}, \nu\right)=F_{1}\left(-q^{2} / M \nu\right)
\end{gathered}
$$

Die am SLAC ${ }^{2}$ Ende der 60er- Jahre gemessenen Strukturfunktionen [7] bestätigen diese Aussage bis auf kleine Abweichungen eindrucksvoll (vgl. Abb.:2.2). Über die Callan-Gross-Relation sind $F_{1}$ und $F_{2}$ für Fermionen im Bjorken-Limes folgendermaßen verknüpft [8]:

$$
2 x F_{1}(x)=F_{2}(x)
$$

Eine Interpretation des Skalenverhaltens wird im Parton-Modell gegeben [9]. Eine Unabhängigkeit der Strukturfunktionen von $Q^{2}$ deutet auf strukturlose Konstituenten (Partonen) des Nukleons hin. Die Streuung des Sondenteilchens erfolgt nicht kohärent am ganzen Nukleon, sondern inkohärent an den Partonen (vgl. Abb.:2.3), wobei das virtuelle Photon an die Ladung eines Partons koppelt. Nimmt man an, daß die Partonen einen zu vernachlässigenden Transversalimpuls zur Richtung des Gesamtimpulses des Nukleons besitzen, koppelt das virtuelle Photon an ein Parton

\footnotetext{
${ }^{2}$ Stanford Linear Accelerator Center
} 


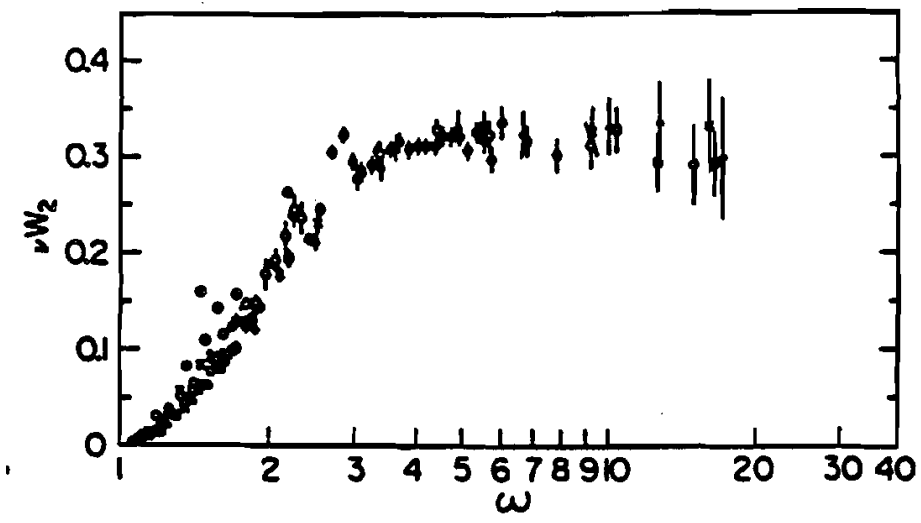

- $E=7.0 \mathrm{GeV}$

Q E $=11.0 \mathrm{GeV}$

* E=13.5 GeV

$E=15.2 \mathrm{GeV}$

+ E=17.7 GeV

Abbildung 2.2: $\nu W_{2}$ gegen $\omega\left(\omega=1 / x_{B j}\right)$ für festen Elektronenstreuwinkel $\left(\theta=10^{\circ}\right)$ und $E_{0}$ im Bereich von 7-17.7 GeV gemessen am SLAC [7]

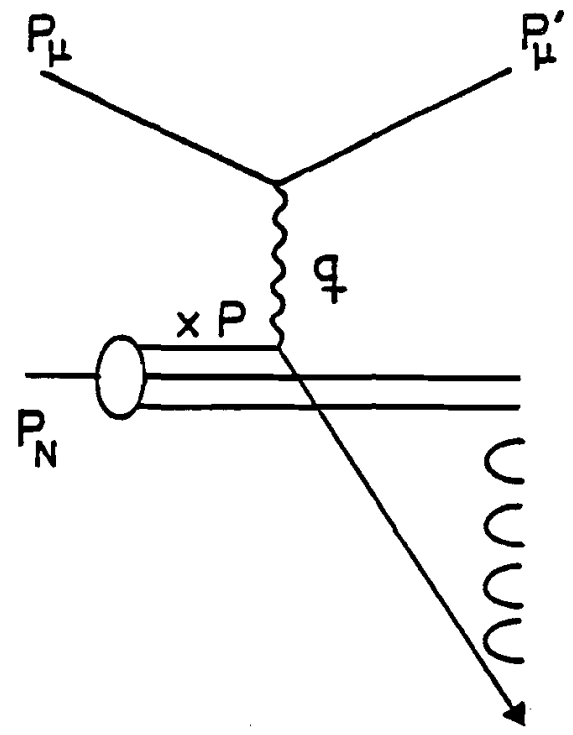

Abbildung 2.3: $\mu$-Nulileon-Streuprozeß im Parton-Modell 
mit dem Impulsbruchteil ${ }^{3} x$. Die Strukturfunktion $F_{2}$ schreibt sich im Parton-Modell als die Summe über die mit dem Ladungsquadrat und Impulsbruchteilen gewichteten Impulsdichteverteilungen $\left(q_{i}(x)\right)$ der Partonen.

$$
F_{2}(x)=\sum_{i} z_{i}^{2} x q_{i}(x)
$$

Detaillierte Messungen der Parton-Eigenschaften in Lepton-Nukleon-Streuexperimenten [10] erwiesen sich als konsistent mit dem Parton-Modell und führten darüberhinaus zur Identifilation der Partonen mit den von Gell-Mann [11] und Zweig hypothetisch eingeführten Quarks, den elementaren Bausteinen der Hadronen.

Die aus am SLAC und Fermilab genommenen Daten extrahierten Quarkverteilungsfunktionen sind in Abb.:2.4 dargestellt [12]. Für große $x(x>0.3)$ findet die Streuung
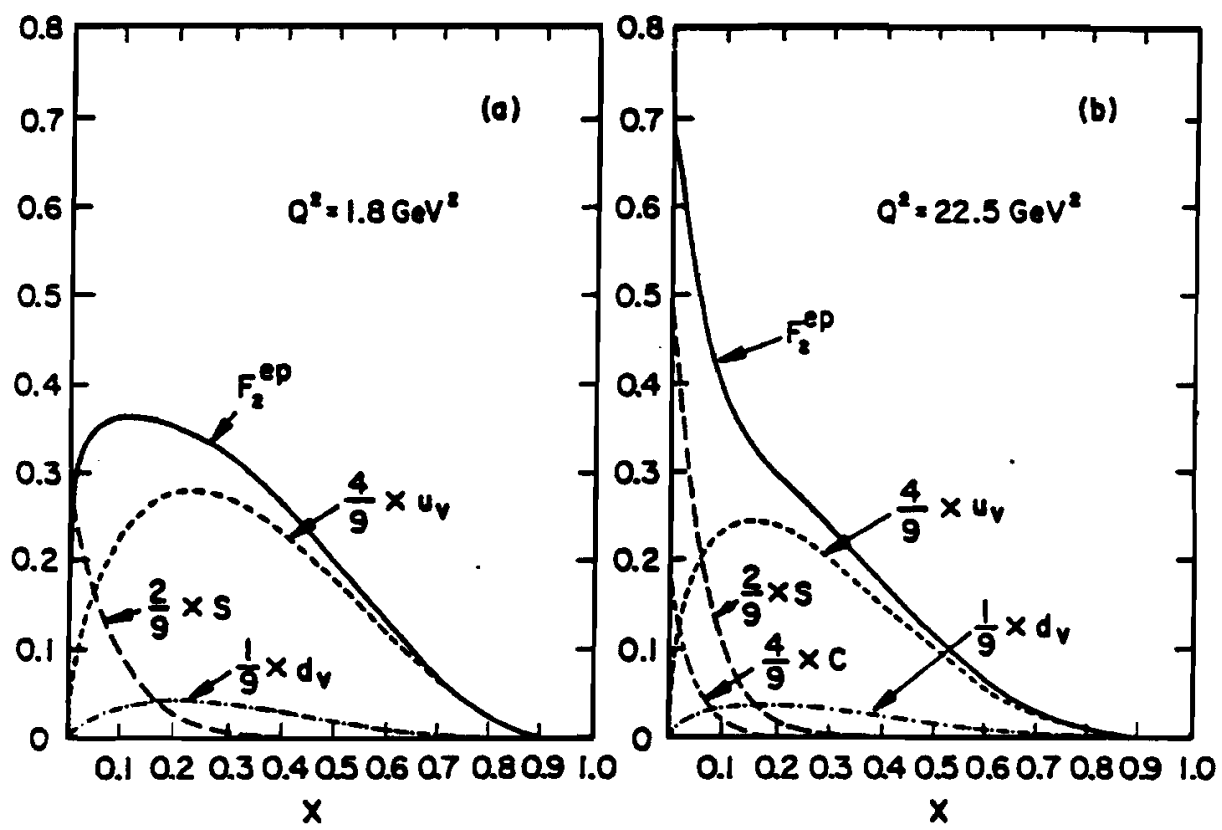

Abbildung 2.4: Quarkdichteverteilungen im Proton nach [12], a) $Q^{2}=1.8(\mathrm{GeV} / \mathrm{c})^{2}$, b) $Q^{2}=22.5(\mathrm{GeV} / \mathrm{c})^{2}$

im wesentlichen an den (Valenz-4) Quarks statt. Für kleiner werdende $x$ gewinnt die Streuung am ladungssymmetrischen See immer mehr an Bedeutung.

Gleichzeitig fallt ein deutlicher Unterschied zwischen Abb.: 2.4 a) $\left(Q^{2}=1.8(\mathrm{GeV} / \mathrm{c})^{2}\right)$ und b) $\left(Q^{2}=22.5(\mathrm{GeV} / \mathrm{c})^{2}\right)$ auf. Die Strukturfunktion $F_{2}$ ist (im Bjorken-Limes)

\footnotetext{
${ }^{3}$ Unter Vernachlässigung der Quadrate der Proton- und Parton-Ruhemassen folgt aus $\left(x P_{P}+q\right)^{2}$ $x=q^{2} / 2 M_{P} \nu \equiv x_{B j}$.

${ }^{4}$ Valenz-Quarks : Quarks, die die Quantenzahlen des Nukleons bestimmen
} 
nicht unabhängig von $Q^{2}$, wie ursprünglich angenommen, sondern steigt mit wachsendem $Q^{2}$ bei kleinem $x$ an und fallt bei großem $\times$ ab. Die Daten der EMC ${ }^{5}$ zeigen deutlich diese Skalenbrechung (Abb.:2.5) [13]. Dieses Verhalten von $F_{2}$ kann im
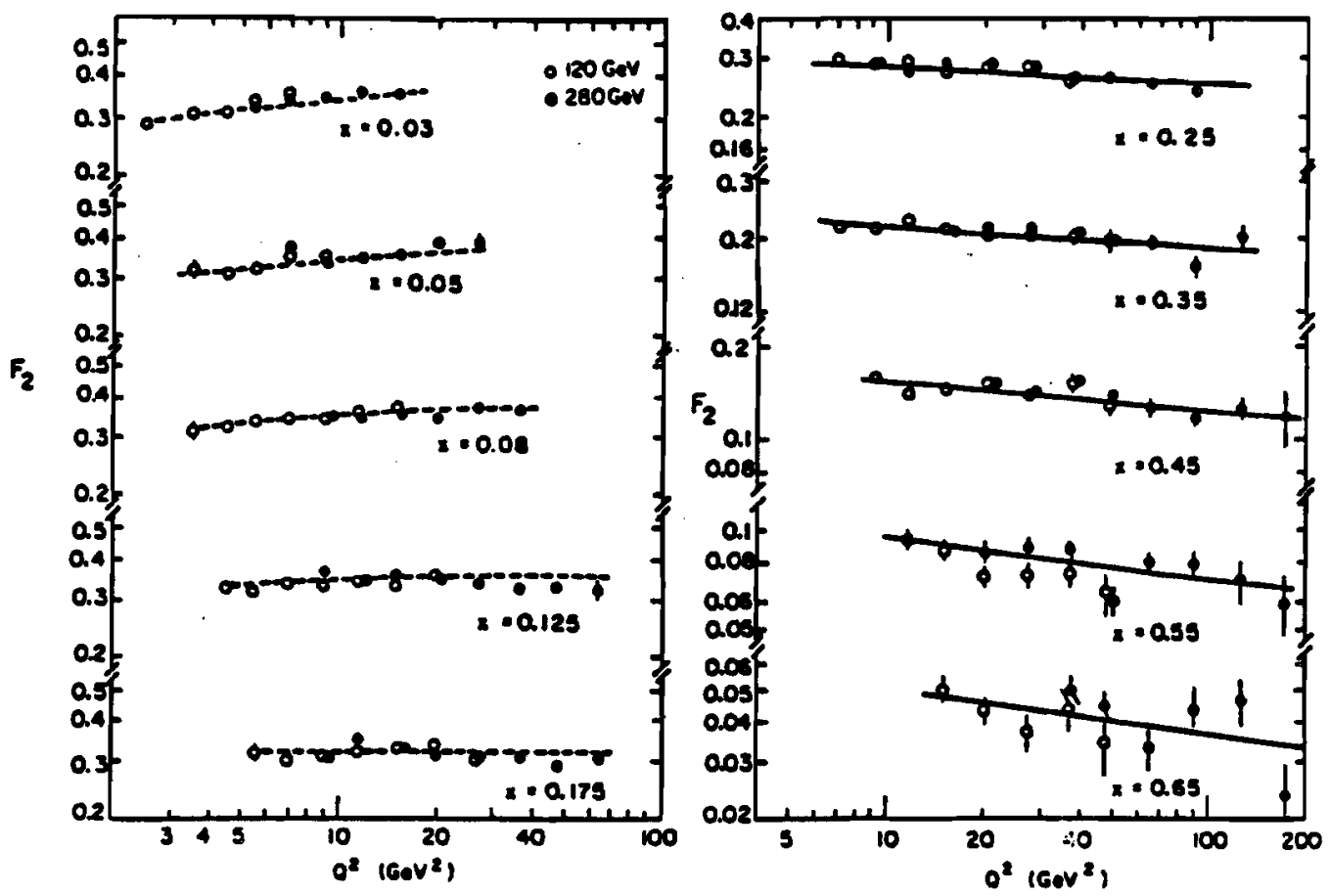

Abbildung 2.i: $F_{2}^{\mu p}$ (EMC) gegen $Q^{2}$ für verschiedene $x$-Bins (aus [13])

Rahmen der Quantenchromodynamik (QCD), der favorisierten Theorie der starken Wechselwirkung, interpretiert werden.

\subsection{Das erweiterte Quark-Parton-Modell}

Die Quarks werden im Nukleon über den Austausch von Gluonen, den Eichbosonen der QCD, gebunden. Die Kopplung erfolgt an die Farbladung der Quarks. Die Stärke der Kopplung wird hierbei (in erster Ordnung) beschrieben durch $\alpha_{s}\left(Q^{2}\right)$ :

$$
\alpha_{s}\left(Q^{2}\right)=\frac{12 \pi}{\left(33-2 n_{f}\right) \ln \frac{Q^{2}}{\Lambda^{2}}}
$$

$n_{f}:$ Anzahl der Quark Flavors

\footnotetext{
${ }^{5}$ EMC : European Muon Collaboration
} 
$\Lambda$, der einzige freie Parameter (neben den Quarkmassen) der QCD wurde experimentell zu $\sim 200 \mathrm{MeV}$ bestimmt [14]. Mit zunehmendem $Q^{2}$ nimmt die Stärke logarithmisch ab (asymptotic freedom). Die elektrisch neutralen Gluonen tragen selbst eine Farbladung und können daher untereinander koppeln (Abb.:2.6c)). Die QCD-

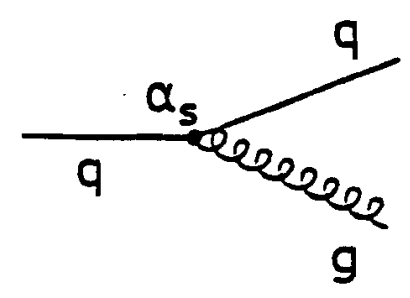

a)

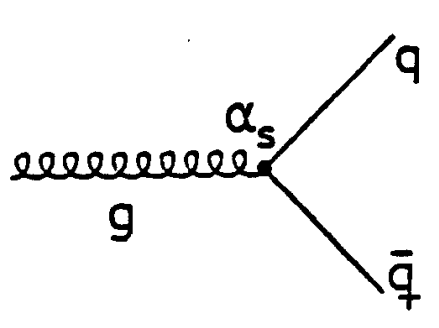

b)

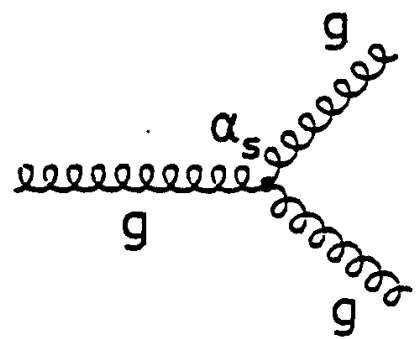

c)

Abbildung 2.6: QCD-Prozesse in erster Ordnung $\alpha_{s}:$ a) Gluon-Bremsstrahlung, b) $q \bar{q}$ Paarbildung und c) Cluon-Selbstkopplung

Splitting-Prozesse in Abbildung 2.6 a) der Gluon-Bremsstrahlung und b) Paarbildung finden ihr Analogon in der QED , die Selbstkopplung c) des ladungsneutralen Photons ist dort jedoch nicht möglich.

Die Änderung der Strukturfunltionen mit $Q^{2}$ lassen sich qualitativ so verstehen [15], daß mit zunehmendem $Q^{2}$ immer kleinere Strukturen im Kern erkennbar und Prozesse der oben angegebenen Art beobachtbar werden.

Wird ein Gluon von Valenz-Quarks abgestrahlt,äußert sich dies in einem stärkeren Abfall von $F_{2}$ zu großen $x_{B j}$. Die relative Zunahme von $q \bar{q}$-Paaren führt zu einer Anreicherung von Quarks mit kleinen Impulsbruchteilen und somit zu einen Anstieg von $F_{2}$ bei kleinen $x_{B j}$. Dies entspricht dem im Experiment beobachteten Verhalten der Skalenbrechung. Qualitativ wird die Variation der Strukturfunktionen von $Q^{2}$ durch die Splittingfunktionen von Altarelli und Parisi beschrieben [16].

Bisher wurde nur auf Prozesse eingegangen, die am leptonischen Vertex beobachtbar sind. Im folgenden Abschnitt soll kurz skizziert werden, wie sich im Quark-PartonModell (QPM) die weitere Entwicklung des gestoßenen Quarks vollzieht. 


\subsection{Die Quarkfragmentierung im Quark-Parton-Modell}

Betrachtet man den Stoßprozeß im Schwerpunktsystem, entfernt sich das gestoßene Quark in entgegengesetzte Richtung zum verbleibenden Targetrest. Je höher die invariante Masse des hadronischen Systems ist, desto deutlicher kann die Fragmentierung des gestoßenen Quarks (Stromfragmentierung) von der des Targetrestes (Targetfragmentierung) unterschieden werden [17]. Die im Fragmentierungsproze $B$ des gestoßenen Quarks gebildeten Hadronen formieren sich in einer bevorzugten Richtung (Jet-Struktur, Vorwärts-Jet; vgl. Abschnitt 2.5).

Im QPM wird angenommen, daß die Fragmentierung unabhängig vom zugrundeliegenden Produktionsprozeß ist und nur vom Energiebruchteil $\left(z_{h}\right)$ der Hadronen am Energieübertrag $\nu$ abhängt (Feynman-Scaling). Die z-Abhängigkeit der Fragmentierung eines Quarks vom Typ $\mathrm{i}$ in ein Hadron $\mathrm{h}$ wird durch die Fragmentationsfunktion $D_{i}^{h}(z)$ beschrieben.

Diese mit dem Begriff enviromental independence gekennzeichnete Prozeßunabhängigkeit der Fragmentierung findet sich in der Formulierung des auf den totalen Wirkungsquerschnitt normierten differentiellen hadronischen Produktionsquerschnittes wieder:

$$
\frac{1}{\sigma_{\text {tot }}} \frac{d \sigma^{h}}{d z}=\frac{1}{N_{\text {event }}} \frac{d N^{h}}{d z}=\sum_{i=u, d, \ldots} \epsilon_{i} D_{i}^{h}(z)
$$

Hierbei gibt $\epsilon_{i}$ die Wahrscheinlichkeit an, daß das fragmentierende Quark vom Typ i ist. $\epsilon_{i}$ ist dabei die einzige prozeßabhängige Größe. Für die Elektroproduktion wird die Wahrscheinlichkeit durch die mit dem Ladungsquadrat gewichteten Quarkverteilungsfunktionen im Nukleon bestimmt: $\epsilon_{i}=z_{i}^{2} q_{i}(x) / \sum_{i} z_{i}^{2} q_{i}(x)$. Die gesamte $x_{B j-}$ Abhängigkeit der z-Verteilungen ist nur in den $\epsilon_{i}$ enthalten (Faktorisierung). In der $e^{+} e^{-}$-Annihilation ist $\epsilon_{i}$, abgesehen von Massen-Schwellen-Effekten, nur abhängig von den Quarkladungen: $\epsilon_{i}=z_{i}^{2} / \sum_{i} z_{i}^{2}$.

Bisher wurden in den Betrachtungen keine QCD-Effekte berücksichtigt. Im erweiterten QPM erwartet man aufgrund der Prozesse wie z.B. der Gluon-Bremsstrahlung: (vgl. Abb.:2.7 a)) eine $Q^{2}$-Abhängigkeit der Fragmentierungsfunktionen $\left(D_{i}^{h}(z) \rightarrow\right.$ $\left.D_{i}^{h}\left(z, Q^{2}\right)\right)$. QCD-Korrekturen wie z.B. die Vertexkorrekur (Abb.:2.7 b)) führen darüberhinaus zur Brechung der faktorisierenden Form der z-Verteilung [18] und

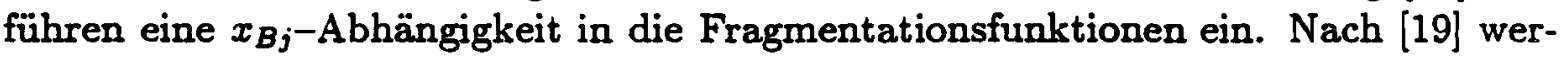
den diese Korrekturen näherungsweise in einem additiven Term zum Hadronproduktionsquerschnitt berücksichtigt. 


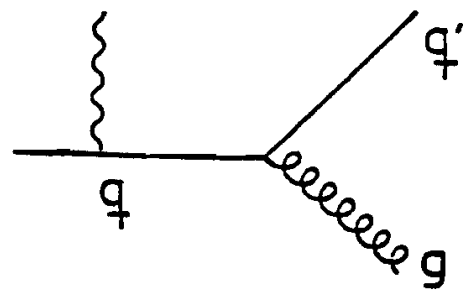

a)

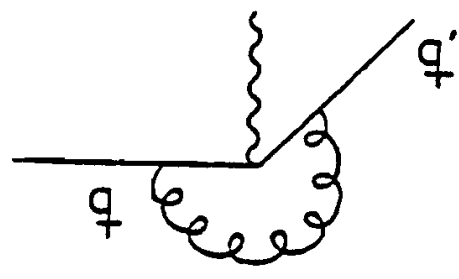

b)

Abbildung 2.7: QCD-Korrelturen, die exemplarisch zur a) Skalenbrechung und b) Faktorisierungsbrechung führen

$$
\frac{1}{\sigma_{t o t}} \frac{d \sigma^{h}}{d z}=\sum_{i=u, d, \ldots} \epsilon_{i}\left(x_{B j}, Q^{2}\right) D_{i}^{h}\left(z, Q^{2}\right)+F_{c o r r}\left(x_{B j}, Q^{2}, z\right)
$$

Qualitativ erwartet man aufgrund der Abstrahlung eines Gluons vom gestoßenen Quark ein Abflachen der z-Verteilung bei hohen z, und aufgrund der zusätzlichen Gluonfragmentierung einen Anstieg der differentiellen Multiplizität bei kleinen z.

Eine weiterführende Diskussion des Verhaltens der longitudinalen und insbesondere der transversalen Impulsverteilungen des hadronischen Endzustandes im Rahmen des QPM's und im Vergleich zu Experimenten und Modellen erfolgt im Kapitel 6.

\subsection{Phänomenologische Monte-Carlo-Modelle zur Fragmen- tierung}

Es wird angenommen, daß das Standardmodell für starke und elektroschwache Prozesse die Information zur detaillierten Beschreibung der Hadronproduktion beinhaltet. Allerdings erscheint es zur Zeit nicht möglich, diese Informationen aus der Theorie zu extrahieren. Zum einen sind die Ereignisse und die unterliegende Anzahl der Einzelprozesse sehr komplex, zum anderen lassen sich Methoden der perturbativen QCD nur auf sehr kleine Abstände auf partonischem Niveau anwenden (ct $1 \mathrm{fm}$ ). Der Übergang vom 'farbigen' Parton in Farb-Singulett-Hadronen, d.h. der Fragmentierungsproze $B$ ist im Zusammenhang mit den non-perturbativen confinement ${ }^{6}$ Eigenschaften der QCD zu sehen [23].

\footnotetext{
${ }^{6}$ Freie Quarks wurden bisher nicht beobachtet [20],[21]. Experimente mit abweichenden Ergebnissen [22] konnten nicht bestätigt werden.
} 
Zur Beschreibung des Fragmentationsprozesses wurden aus diesen Gründen phänomenologische Modelle entwickelt. In der zeitlichen Abfolge entstanden im wesentlichen drei Typen von Modellen: Independent Fragmentation Models (IF), String Fragmentation Models (SF) und Cluster Fragmentation Models (CF).

Im Field-Feynman-Modell [24], aus dem sich verschiedene IF-Modelle entwickelt haben, wird folgender iterativer Ansatz ausgeführt: Ein angeregtes primäres Quark (a) mit bestimmter Energie und Impuls erzeugt ein Farbfeld, in dem neue $q \bar{q}$-Paare gebildet werden. Quark a kombiniert mit dem Antiquark $\bar{b}$ aus einem erzeugten Quark- Antiquark-Paar ( $b \bar{b})$ zu einem (angeregten) Meson. Das verbleibende Quark b formiert mit einem weiteren Antiquark ( $\bar{c})$ wiederum ein Hadron .... (vgl. Abb.: 2.8). Einige der Mesonen können ihrerseits zerfallen. Im Fragmentierungsproze $\$$

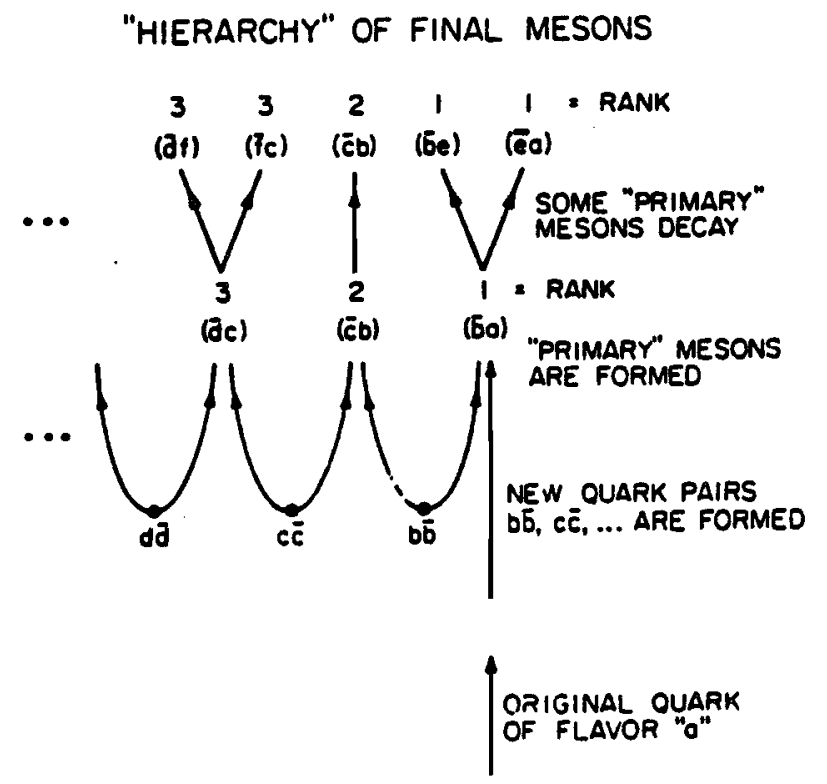

Abbildung 2.8: FragmentierungsprozeB im Field-Feynman-Modell [24]

erfolgt die Energieaufteilung entsprechend einer Wahrscheinlichkeitsverteilung $f(z)$, wobei $f(z)$ die Energie des Hadrons ist und (1-z) die des verbleibenden Quark-Jets. Die (skalierte) Verteilungsfunktion wird für jeden iterativen Schritt gleich angenommen, d.h. unabhängig von der verbleibenden Energie. In der $\mathrm{e}^{+} \mathrm{e}^{-}-$Vernichtung, in der die $q \bar{q}$-Paare in entgegengesetzte Richtungen propagieren und anschließend hadronisieren, wurde diese Idee der Einzel-Jet-Fragmentierung zur Beschreibung von Zwei-Jet-Ereignissen erweitert. Hierbei erfolgt die Fragmentierung der Jets zunächst unabhängig voneinander. Farb-, Impuls- und Energieerhaltung werden nachträglich eingeführt.

Im Gegensatz hierzu wird, in einem von der Lund-Gruppe entwickelten StringFragmentierungsmodell, ein Raum-Zeit-Bild verwendet [25]. In $\operatorname{der} \mathrm{e}^{+} \mathrm{e}^{-}-$Annihilation 
bewegen sich das erzeugte $q$ und $\bar{q}$ in entgegengesetzter Richtung auseinander, in der Leptoproduktion das gestoßene Quark und der Target-(Diquark-) Rest. Zwischen den farbigen Partonen spannt sich eine 'color flux tube' auf. Die transversale Ausdehnung dieser Farbflußröhre beträgt etwa $1 \mathrm{fm}$, entsprechend der typischen Ausdehnung der Hadronen. Nimmt man die Energiedichte entlang der Farbflußröhre als konstant an, führt dies zu der Vorstellung des (linearen) confinement. Eine lorentzinvariante Beschreibung der Dynamik erfogt hierbei mittels 'masseloser' relativistischer Strings, die keine transversale Anregung besitzen. Das Potential entlang des String nimmt linear mit dem Abstand $z u$, wobei die Stringspannung $\kappa$ mit $1 \mathrm{GeV} / \mathrm{fm}\left(=0.2 \mathrm{GeV}^{2}\right)$ angenommen wird [26]: $V(x)=\kappa * x$.

Je weiter sich die Quarks voneinander entfernen, desto größer wird die potentielle Energie im String. Er bricht auf und ein $q_{1} \overline{q_{1}}-$ Paar wird erzeugt (vgl. Abb.: 2.9).

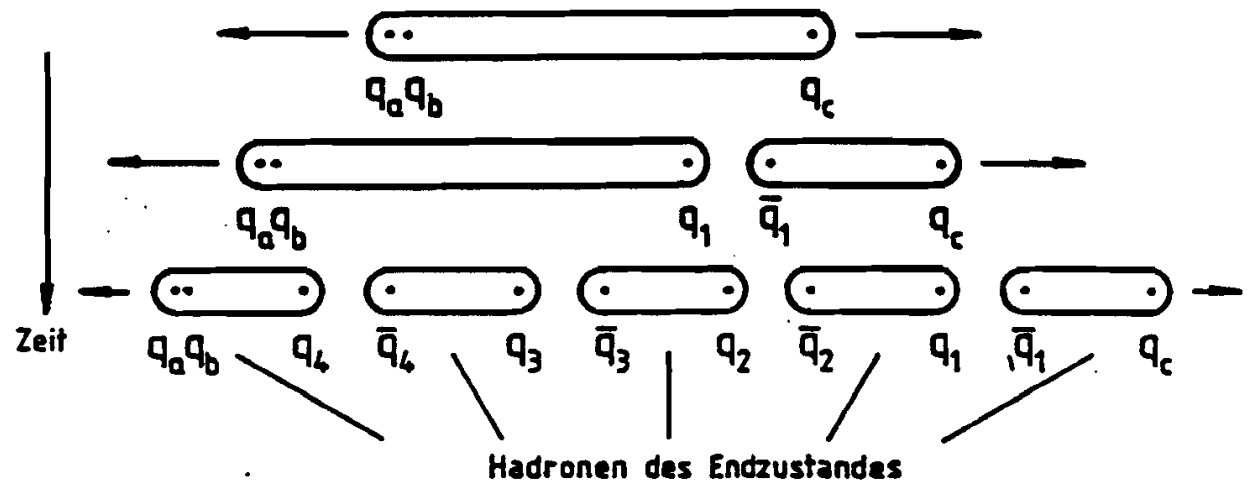

Abbildung 2.9: String-Fragmentierung im Lund-Modell [27].

Ist die invariante Masse eines Subsystems hinreichend groß, erfolgen weitere Aufbrüche des String in $q_{i} \bar{q}_{i}$-Paare. Im Endzustand enstehen so die beobachtbaren farbneutralen Hadronen. Die Baryonproduktion ist in ähnlicher Weise implementiert. In der einfachsten Form werden anstelle der Quark-Antiquark-Paare Diquark-AntidiquarkPaare gebildet, die mit einem Antiquark (Quark) Baryonen bilden. In jeden Schritt des Fragmentierungsprozesses sind Flavor, Energie und Impuls erhalten.

Die an einem Raum-Zeit-Punkt kreierten $q \bar{q}-$-Paare erhalten aufgrund des quantenmechanischen Tunneleffektes eine transversale Masse $\mathrm{m}_{t}$, wobei die Produktionswahrscheinlichkeit proportional ist zu:

$$
\exp \frac{-\pi m_{l}^{2}}{\kappa}=\exp ^{\frac{-\pi m^{2}}{\kappa}} \exp \frac{-\pi p_{s}^{2}}{\kappa}
$$

Dieser in Masse und Transversalimpuls faktorisierende Term führt zu eincm gaußförmigen, flavor-unabhängigen $P_{T}$-Spektrum. Darüber hinaus folgt eine Unterdrückung 
der Produktion von schweren Quarks: $u: d: s: c \approx 1: 1: 0.3: 10^{-11}$. Da der String keine transversale Anregung besitzt, wird der Transversalimpuls der $q \bar{q}-\mathrm{Paare}$ lokal kompensiert.

Einen zusätzlichen Beitrag zum Transversalimpuls erhalten die Partonen aufgrund der Gluonabstrahlung. Gluonen sind im Bild des String-Modells folgendermaßen implementiert: Der String zwischen Quark und Antiquark (bzw. zwischen Quark und Diquark in der Leptoproduktion) bildet einen 'Knick', der ein Gluon mit bestimmter Energie und Impuls repräsentiert. Das Gluon erhält damit die zweifache Farbladung gegenüber dem $q$ und $\bar{q}$ an den Enden des String. Bricht der String im Bereich des Gluon auf, fragmentieren die verbleibenden beiden Stringreste in oben beschriebener Weise. Zusätzlich fragmentiert das Gluon in Hadronen. Aufgrund der Vorstellung eines geknickten String erwartet man eine höhere Teilchendichte im Bereich zwischen Gluon und Quark (Antiquark) gegenüber dem Winkelbereich zwischen Quark und Antiquark (Stringeffekt). Diese Asymmetrie, die von IF-Modellen nicht vorhergesagt wird, wurde von der Jade-Gruppe im Experiment tatsächlich beobachtet [28].

Modelle dieser Art, die auf QCD-Annahmen in erster und zweiter Ordnung Störungsrechnung basieren, konnten allerdings nicht alle Datenverteilungen überzeugend beschreiben. So wird z.B. die Mehrfachjetrate unterschätzt.

Dies führte u.a. zur Entwicklung von Modellen mit sukzessiver Aufspaltung auf partonischem Niveau. Dieser Verzweigungsprozeß kann mit Hilfe der Altarelli-ParisiGleichungen beschrieben werden [16]. Diese geben die Wahrscheinlichkeit für die Prozesse $q \rightarrow q g, g \rightarrow g g$ und $g \rightarrow q \bar{q}$ an.

$$
\frac{d P_{a \rightarrow b c}}{d t}=\int d z \frac{\alpha_{s}\left(Q^{2}\right)}{2 \pi} P_{a \rightarrow b c}(z)
$$

Der Verzweigungsproze $B$ findet solange statt, bis die Partonmasse eine untere Massenschwelle $\mathrm{m}_{\min }$ erreicht hat (vgl. Abb.:2.10). Eine anschließende Hadronfragmentierung nach dem Prinzip der IF- und SF-Modelle ist möglich. Läßt man jedoch alle Gluonen in Quark- Antiquark-Paare zerfallen, können Farb-Singlet-Subsysteme (Kluster) gebildet werden. In den Kluster-Fragmentationsmodellen werden keine weiteren Aussagen über die innere Struktur der Kluster gemacht. Bedeutung haben nur die Masse und die Flavorquantenzahlen. Die Kluster zerfallen isotrop (in ihrem Ruhesystem) in beobachtbare Hadronen (Abb.: 2.11). 


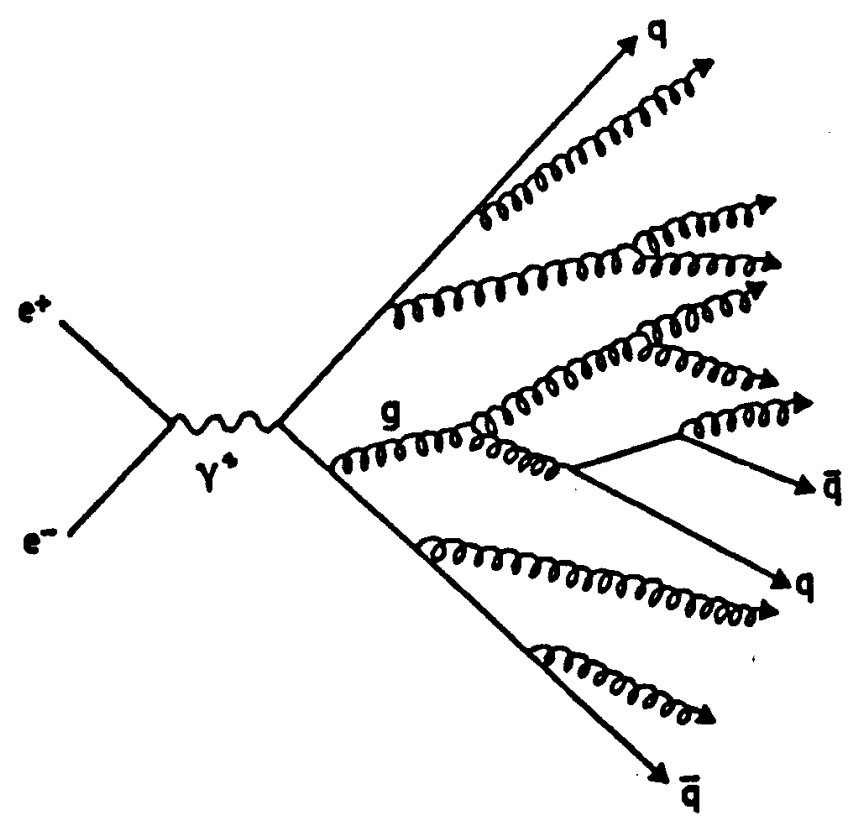

Abbildung 2.10: Partonschauerentwicklung am Beispiel der $\mathrm{e}^{+} \mathrm{e}^{-}-$Annihilation [23].

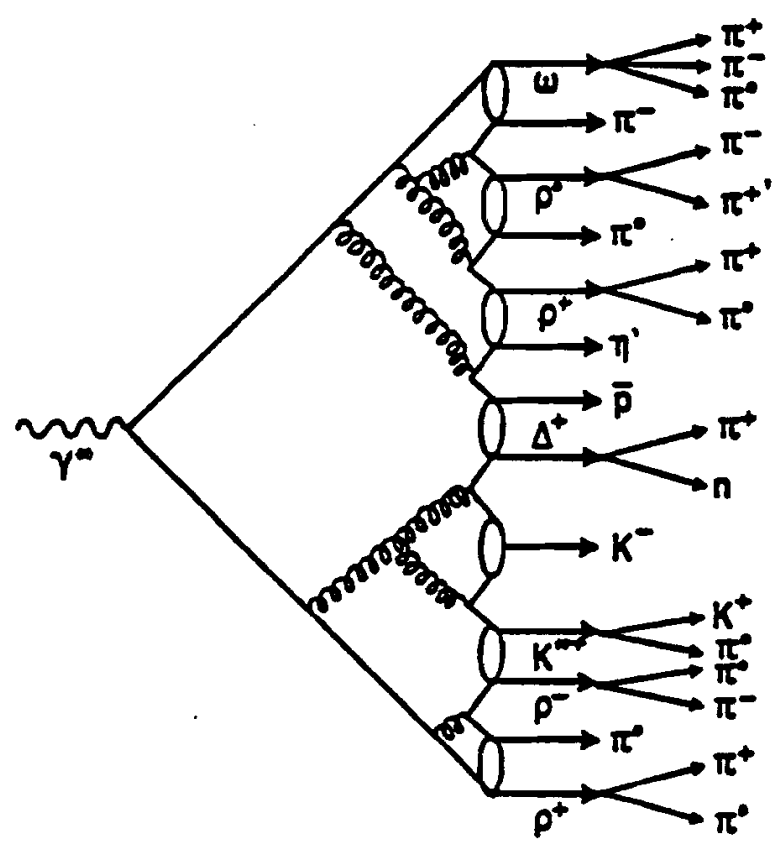

Abbildung 2.11: Hadronisierung im K̈luster-Fragmentierungs-Modell [23]. 


\section{Das Experiment 665 am Fermilab}

Das Fermi National Accelerator Laboratory in Batavia, Illinois, USA ist eines der großen Hochenergiephysik Forschungszentren der Welt. Der Bau des Laboratoriums begann im Jahr 1967, am 1. März 1972 wurden dort Protonen erstmalig auf 200 $\mathrm{GeV}$ beschleunigt [29]. Seitdem wurden dort ca. 300 Experimente durchgeführt [30]. Die wesentlichen Forschungsaktivitäten im experimentellen Bereich schlüsseln sich auf in:

1. $p \bar{p}$ Collider Physik

2. Fixed-Target Experimente

3. Beschleuniger-Physik

Im MeBprogramm wechseln sich Collider (Tevatron I) und Fixed-Target Experimente (Tevatron II) zyklisch ab. Darüberhinaus ist bemerkenswert, daß im Rahmen der Nuklearmedizin ein Neutronentherapie-Zentrum auf dem Gelände des Fermilab eingerichtet wurde, und zur Zeit ein 70-250-MeV-Protonenbeschleuniger zur Krebsbehandlung entwickelt und gebaut wird [31].

\subsection{Das Tevatron}

Das Kernstück der Anlage bildet der zwei Kilometer durchmessende $p \bar{p}$-DoppelBeschleunigungsring, in dem Protonen und gegenläufig Antiprotonen auf eine Endenergie von ca. $1 \mathrm{TeV}$ beschleunigt werden können. Die Erzeugung und Beschleunigung der Protonen wird im folgenden kurz skizziert.

Wasserstoffatome werden in einem Cockcroft-Walton-Generator ionisiert und im elektrischen Feld auf $0.75 \mathrm{MeV}$ vorbeschleunigt. In einem anschließenden Linearbeschleuniger werden die Protonen auf eine Energie von $200 \mathrm{MeV}$ gebracht. Eine weitere Vorbeschleunigung im Booster-Ring erhöht die Energie der Protonen auf 8 $\mathrm{GeV}$, die ausreichend ist, die Partikel in den Hauptbeschleuniger zu injizieren [29]. Der Beschleuniger besteht aus einer übereinander gelagerten Doppelringstrukt ur [32]. Im oberen (mit konventionellen Magneten ausgestatteten älteren) Teil (Hauptring) erfolgt die Beschleunigung der Teilchen auf max. $150 \mathrm{GeV}^{1}$, im 1983 erstmalig in

\footnotetext{
${ }^{1}$ Vor dem Ausbau wurden Protonen im Hauptring auf maximal $500 \mathrm{GeV}$ gebracht.
} 
Betrieb genommenen unteren Teil (Tevatron) werden sie in sechs Beschleunigungsstrecken auf ihre Endenergie gebracht. Die ca. 1000 Ablenk- und Fokussierungsmagnete im neueren Teil sind supraleitend aufgebaut [33] und ermöglichen hierdurch die maximale Endenergie von $1 \mathrm{TeV}$. Dies ist die zur Zeit höchste Energie, auf die Protonen in Laboratorien beschleunigt werden können.

Tabelle 3.1 enthält exemplarisch einige Kenngrößen des Tevatrons für den FixedTarget Operationsmodus.

\begin{tabular}{|ll|}
\hline & \\
Beschleunigerradius & $1 \mathrm{~km}$ \\
Maximale Strahlenergie & $800-1000 \mathrm{GeV}$ \\
Injektionsenergie & $150 \mathrm{GeV}$ \\
Intensität & $\sim 2 \cdot 10^{13}$ Protonen/ Zyklus \\
Zylluszeit & $60 \mathrm{sec}$ \\
Spill-Dauer ('slow spill') & $20 \mathrm{sec}$ \\
Hochfrequenz & $53 \mathrm{MHz}$ \\
\hline
\end{tabular}

Tabelle 3.1: Tevatron Parameter

Die Erzeugung der Antiprotonen erfolgt für den Fall einer Collider-Meßperiode vereinfacht dargestellt folgendermaßen: Im Hauptring werden Protonen auf $120 \mathrm{GeV}$ beschleunigt und anschließend auf ein Wolfram-Target gelenkt. Die im Kollisionsprozeß erzeugten Antiprotonen werden in einen Speicherring (Akkumulator) fokussiert und dort Phasenraum-geformt. Nachdem der SammelprozeB abgeschlossen ist, werden die $\bar{p}$ zurück in den Hauptring transferiert und dort nach einer weiteren Beschleunigungsphase mit $150 \mathrm{GeV}$ in den Tevatron-Ring injiziert. Die gleiche Anzahl von Proton-Teilchenpaketen mit annähernd gleicher Intensität wird im Hauptring bereitgestellt und zusammen mit den Antiprotonen im Tevatron auf eine Endenergie von ca. $1 \mathrm{TeV}$ gebracht [34].

Gegenwärtig ist nur eine der sechs möglichen Wechselwirkungszonen mit einem vollständigen Detektor ausgerüstet - dem Collider Detector at Fermilab (CDF) - ein weiterer Detektor soll zur nächsten Meßperiode einsatzbereit sein (D0).

In der Tevatron-II-Meßperiode werden Protonen aus dem Tevatron ausgelenkt und auf die verschiedenen Experimente verteilt (Switchyard). Je nach experimentellen Anforderungen werden die Protonen direkt zur Streuung geleitet, oder es werden durch sekundäre Wechselwirkungen Teilchenstrahlen der benötigten Teilchensorte in den verschiedenen Strahlführungen (Beamlines) erzeugt und zu den entsprechenden Experimenten geleitet. Abbildung 3.1 gibt einen Gesamtüberblick über die a) Fermilab-Beschleuniger und b) Strahlführungen. 

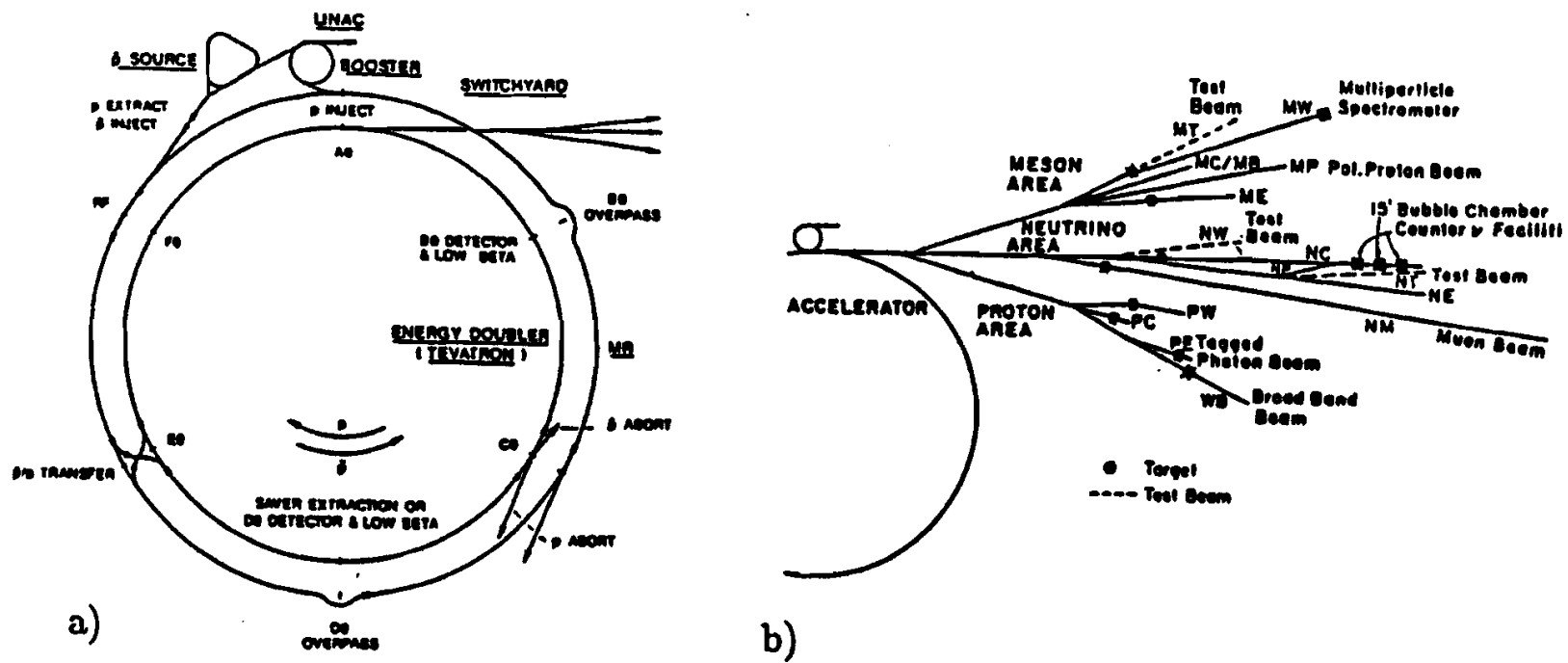

b)

Abbildung 3.1: Gesamtüberblick über die a) Fermilab-Beschleuniger und b) Strahlführungen [32]

\subsection{Die E665-Kollaboration}

Die Kollaboration besteht aus ca. 80 Mitgliedern aus 13 internationalen Instituten (9 USA, 3 Westdeutschland, 1 Polen). Die Teilnehmer aus den entsprechenden Instituten sind in Tabelle 3.2 (Stand: Juni 1989) aufgeführt.

\subsection{Die Strahlführung des Experimentes 665}

In der NM²-Strahlführung am Fermilab wird der Myonstrahl für das Experiment 665 erzeugt [35]. Die vom Switchyard zugeteilten Protonen werden auf ein ca. $50 \mathrm{~cm}$ langes Beryllium-Target gelenkt. Im Proton-Nukleon-Stoßprozeß werden über die starke Wechseiwirkung in der Mehrzahl positive Pionen erzeugt. Ca. 5\% dieser sekundären Teilchen zerfallen in einer $1.1 \mathrm{~km}$ langen fokussierenden, defokussierenden Magnetoptik (FODO) in $\mu$ und $\nu_{\mu}$. Die Absorption der Hadronen und Elektronen erfolgt in einem elf Meter langen Beryllium-Absorber. Durch diesen hindurch gelangen die Myonen in einen zweiten $420 \mathrm{~m}$ langen FODO-Bereich, in dem sie durch $\mathrm{Di}-$ und Quadrupole im Impuls selektiert und auf das Target im Experiment fokussiert werden. Ein $3 \mathrm{mr}$ ablenkender Dipolmagnet im letzten Bereich der Strahlführung wird zur Impulsbestimmung verwendet. Zwei Detektorgruppen vor (Station 1 und 2) und hinter dem Ablenkmagneten (Station 3 und 4), bestehen aus jeweils zwei Ebenen

\footnotetext{
${ }^{2}$ new muon
} 
E665 Collaboration

M.R. ADAMS ", S. AID 8), P.L. ANTHONY "), M.D. BAKER ", J.F. BARTLETT "), A.A. BHATTI "),

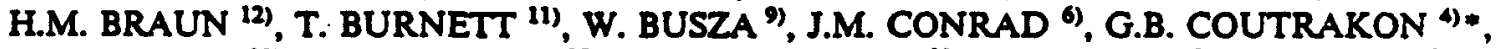
R. DAVISSON ${ }^{11}$, S.K. DHAWAN ${ }^{\text {(3), W. DOUGHERTY }}{ }^{\text {11) }}$, T. DREYER ${ }^{\text {) }}$, U. ECKER ${ }^{12)}$, V. ECKARDT ${ }^{10)}$, M. ERDMANN "), A. ESKREYS ${ }^{3)}$, K. ESKREYS 3), H.J. GEBAUER ${ }^{10)}$, D.F. GEESAMAN "), R. GILMAN 1), M.C. GREEN 1)**, J. HAAS "), C. HALLIWELL "), J. HANLON "), V.W. HUGHES ${ }^{\text {(3) }}$, H.E. JACKSON "), D.M. JANSEN ${ }^{11)}$, G. JANCSO ${ }^{10}$, S. KAUFMAN ", R.D. KENNEDY ${ }^{2)}$, T.B.W. KIRK ()+, H. KOBRAK ${ }^{2)}$, S. KRZYWDZINSKI "1) * *, S. KUNORI "), J. LORD "1), H.J. LUBATTI "1), T. LYONS "), S. MAGILL ", P. MALECKI "), A. MANZ ${ }^{10)}$, D. MCLEOD n, H. MELANSON "), D.G. MICHAEL (), W. MOHR (), H.E MONTGOMERY 4), J.G. MORFIN 4), R.B. NICKERSON ${ }^{6}$, S. O'DAY 8), A.M. OSBORNE 24.6) , L. OSBORNE 9), B. PAWLIK "), F.M. PIPKIN (), EJ. RAMBERG "),

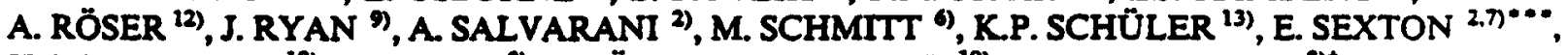
H.J. SEYERIEIN ${ }^{10)}$, A. SKUJA ", S. SÖLDNER-REMBOLD ${ }^{10)}$, P.H. STEINBERG ${ }^{\text {*) }}$, H.E STIER (), P. STOPA ${ }^{10) \&}$, P. STRUBE ${ }^{10)}$, Robert A. SWANSON ${ }^{2)}$, R.I TALAGA ${ }^{\text {8)+ }}$, S. TENTINDO-REPOND ${ }^{1)++}$, H.-J. TROST ${ }^{10)+}$, H. VENKATARAMANIA ${ }^{13)}$, M. VIDAL ${ }^{10}$, M. WILHELM "), I. WIT.KES (1), Richard WILSON "), S. WOLBERS 4) and T. ZHLOO "1)

\footnotetext{
"Argonne National Laboratory, Argonne, IL 60439, USA

") University of Calffornia San Digza La Jolla, CA 92093, USA

3) Institute of Nuclear Physics, Cracow, Poland, and Instirute of Nuclear Physics and Technology, Cracow, Poland

"Fermi National Accelerator Laboratory, Bacooia, IL 6OSIQ, USA

9) Alber-Ludwigs-Universilat, 7800 Freibure 2 Br., FRG

"Harvard University, Cambridge, MA 02138, USA

"Unioersity of Illinois at Chicego, Chicogo, IL 60680, USA

8) University of Maryland, College Park, MD 20712, USA

9) Massachusetis Institute of Technology, Cambridge, MA 02139, USA

10) Max-Planck-Institut fir Physik wed Astrophysik, 8000 Minich 40, FRG

11) University of Washington, Seatule, WA 98195, USA

12) Universitärs-Gesamthochschule Wuppertal, 5600 Wupperal 1, FRG

13) Yale Universig, New Hower, CT 06520, USA
}

Tabelle 3.2: Mitgliederliste der E665-Kollaboranten 
Szintillationsstreifen (SBT's) ${ }^{3}$ und sechs Proportionalkammerebenen (PBT's) (vgl. Abb.:3.2).

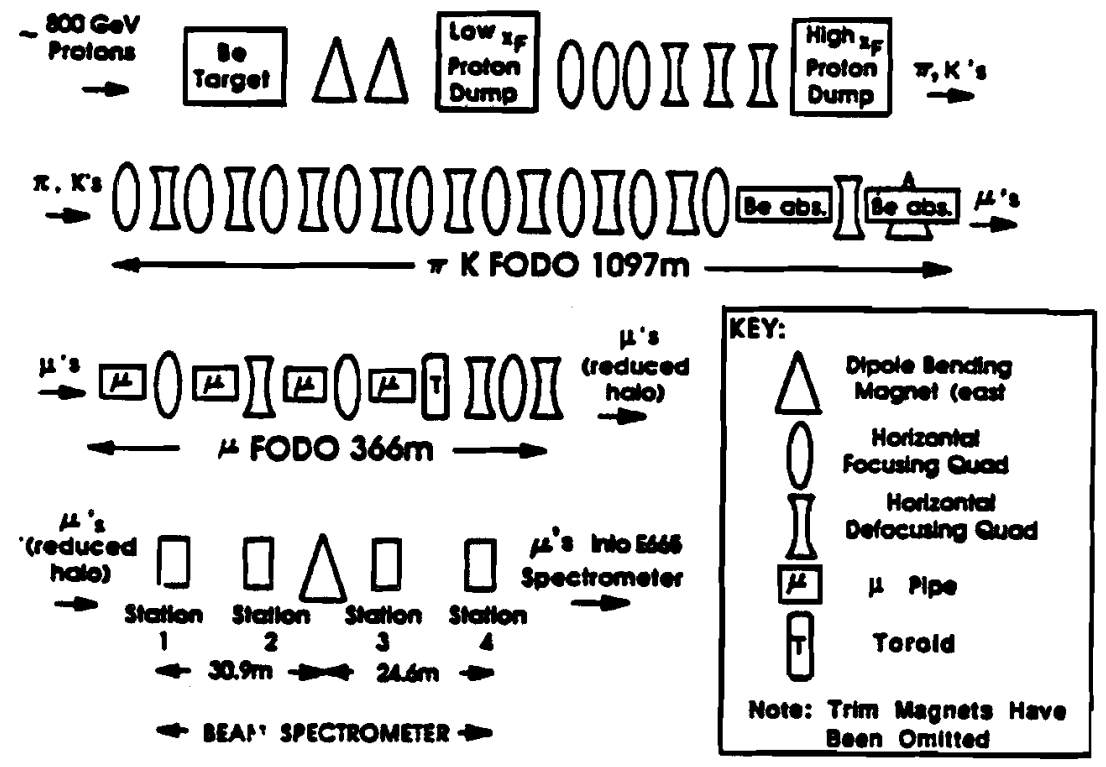

Abbildung 3.2: Schematischer Aufbau der NM-Strahlführung

Die Signale der Hodoskope werden zur Bildung des Beamtriggersignales, die Drahtansprecherinformation zur Rekonstruktion der Strahlspuren verwendet. Abb 3.3 a) zeigt das horizontale und vertikale Strahlprofil am Ort der StrahlmeBstation $4^{4}$, b) die Impulsverteilung für die 500-GeV-Justierung der Strahlführung. In Tabelle 3.3 sind die wesentlichen Parameter aufgelistet. Bemerkenswert ist, daß der Myonstrahl die Hochfrequenzstrultur des Tevatronsystems beibehält. Die Myonen sind hierdurch in 19-nsec-Zeitabständen getrennt ('RF buckets') und ermöglichen damit eine genaue Synchronisation der Triggerimpulse. Weiterhin besteht die Möglichkeit in der Strahlführung Hadronen und Elektronen zum Experiment zu leiten. Elektronenstrahlen niederer Energie (2-150 GeV) wurden insbesondere zur Eichung des

\footnotetext{
3Station 2 nur eine Ebene

${ }^{4}$ Aufgrund der geringen Winkeldivergenz des Myonstrahles sind die Strahlprofile am Ort der Strahlmeßstation 4 und am Target praktisch identisch.

${ }^{3}$ Typischer Wert in der Meßperiode 1987/1988; Anzahl der Protonen, die in die NM-Strahlführung extrahiert wurden. Dies entspricht nur einem Teil der gesamten aus dem Tevatron extrahierten Protonen, da diese am Switchyard auf die verschiedenen Experimente verteilt werden. Die Gesamtzahl der Protonen pro Spill beträgt $\sim 10^{13}$.
} 


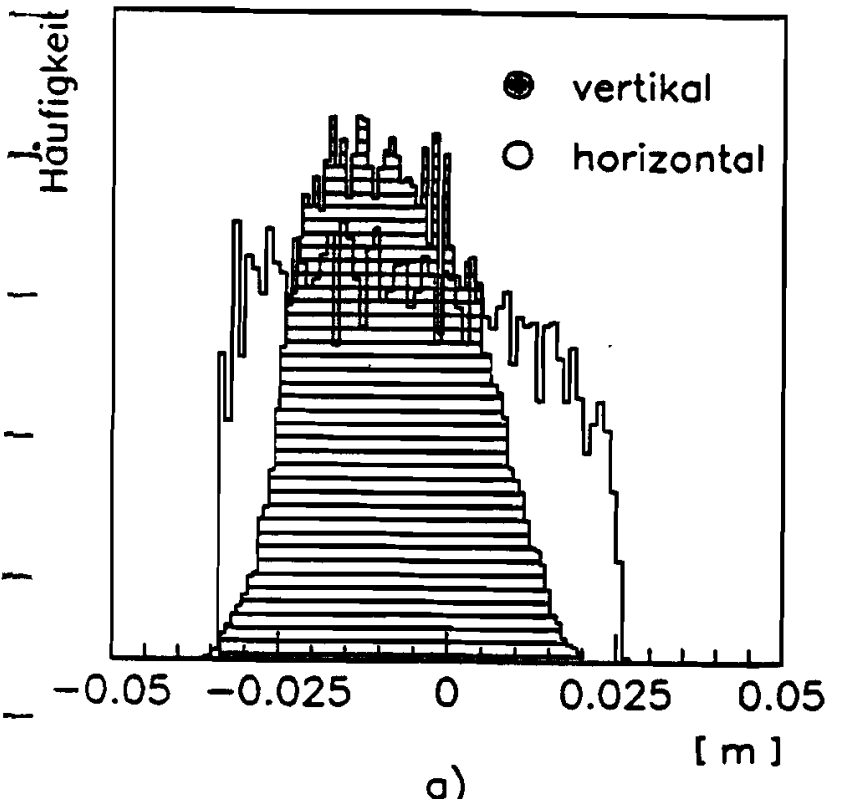

a)

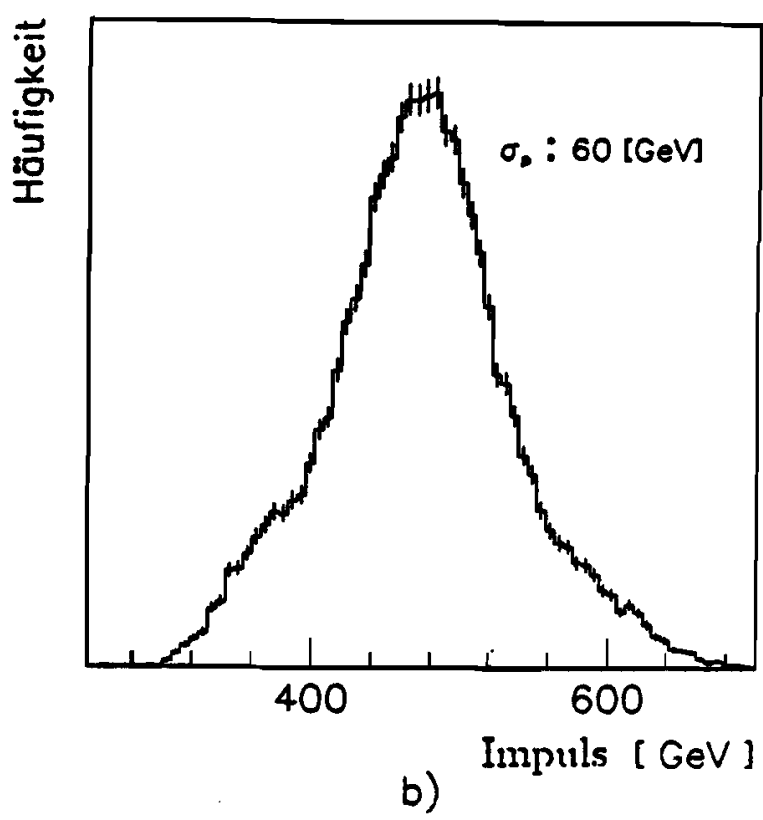

b)

Abbildung 3.3: Das Strahlprofil am Ort der Strahlmeßstation 4. a) horizontales und vertikales Strahlprofil, b) Impulsverteilung für die 500-GeV-Justierung (nach Anbringen der Auswahlkriterien aus Tabelle [5.1])

\begin{tabular}{|ll|}
\hline Anzabl Protonen / Spill & $2.5 \times 10^{12}$ \\
Anzahl Myonen / Proton & $5.5 \times 10^{-6}$ \\
$\sigma_{p}$ & $60 \mathrm{GeV}$ \\
$\frac{\delta_{p}}{p}$ & $\simeq 0.5 \%$ \\
Halo (im Bereich $20 \times 20 \mathrm{~cm}^{2}$ & $10-15 \%$ \\
um nominale Strahlachse) & \\
\hline
\end{tabular}

Tabelle 3.3: Parameter des 500-GeV-Myonstrahles 
Flugzeitmeßsystems und der Cerenkovzähler (große Winkelakzeptanz) sowie des elektromagnetischen Kalorimeters genutzt.

\subsection{Der Detektor des Experimentes 665}

In Abb.: 3.4 ist die Gesamtapparatur dargestellt. Das Spektrometer ist mit zwei gegenpolig betriebenen, supraleitenden Dipolmagneten ausgestattet, deren integrierte Feldstärken derart ausgelegt $\operatorname{sind}^{6}$, daß ein gestreutes $\mu$ (annähernd) impulsunabhängig ins Triggersystem fokussiert wird. Einige Detektorelemente im Vertexbereich ${ }^{7}$ wurden bereits im Experiment NA9 der EMC am CERN ${ }^{8}$ verwendet [36] . Die Detektoren des Vorwärtsspektrometers wurden für dieses Experiment neu konstruiert. Der Chicago Cyclotron Magnet (CCM)[37] wird im E665-Spektrometer zur Impulsbestimmung der hochenergetischen Teilchen benutzt. Die kombinierte Akzeptanz des Vertex- und Vorwärtsspektrometers für geladene Teilchen deckt annähernd den gesamten Raumwinkel ab. Mittels TOF, Cerenkovzählern und einem elektromagnetischen Kalorimeter ist Teilchenidentifikation in einem weiten Impulsbereich ermöglicht. Der Aufbau des Spektrometers wird im Folgenden kurz beschrieben, eine detaillierte Beschreibung des Apparates ist in [38] gegeben.

\subsubsection{Das Target}

Im Experiment wurden Streuungen an Alüssigem Deuterium und Wasserstoff und verdichtetem Xenon-Gas durchgeführt. Die Targets befanden sich innerhalb der Streamerkammer. Dies verbot die Verwendung von elektrisch leitendem Material und verhinderte damit eine 'Superinsulation'. Speziell für die flüssigen Targetmaterialien $H_{2}, D_{2}$, die sich in einem ca. $1.15 \mathrm{~m}$ langen, $9 \mathrm{~cm}$ durchmessenden, thermisch isolierten Captonzylinder befanden, betrug die Wärmeverlustleistung ca. 25 Watt. Der Targetbehälter war mit einem Reservoir verbunden, in dem flüssiger Wasserstoff bzw. Deuterium über eine Dampfdruckregelung mit flüssigem Helium auf konstanter Temperatur gehalten wurde. Aufgrund der hohen Wärmeleitfähigkeit des Wasserstoffes (Deuteriums) und daraus resultierender thermodynamischer Strömung war mit keinem Sieden der Flüssigkeiten zu rechnen. Die Variationen des Dampfdruckes wurden auf einem Schreiber protokolliert und später ausgewertet [39] . Die Dichtevariation für $\mathrm{H}_{2}, \mathrm{D}_{2}$ betrug weniger als $1 \%$ während des gesamten Zeitraumes

\footnotetext{
${ }^{6} \mathrm{CVM}: B=1.5 \mathrm{~T} ; \int B d l=4.3 \mathrm{Tm}, \mathrm{CCM}: B=1.3 \mathrm{~T} ; \int B d l=-6.7 \mathrm{Tm}$

${ }^{7}$ Vertexspektrometermagnet CVM (Cern Vertex Magnet), Streamerkammer SC, Cerenkovcounter $\mathrm{C0}, \mathrm{C} 1$, Time of Flight System TOF (modifiziert)

${ }^{8}$ Conseil Europèen pour la Recherche Nucléaire
} 


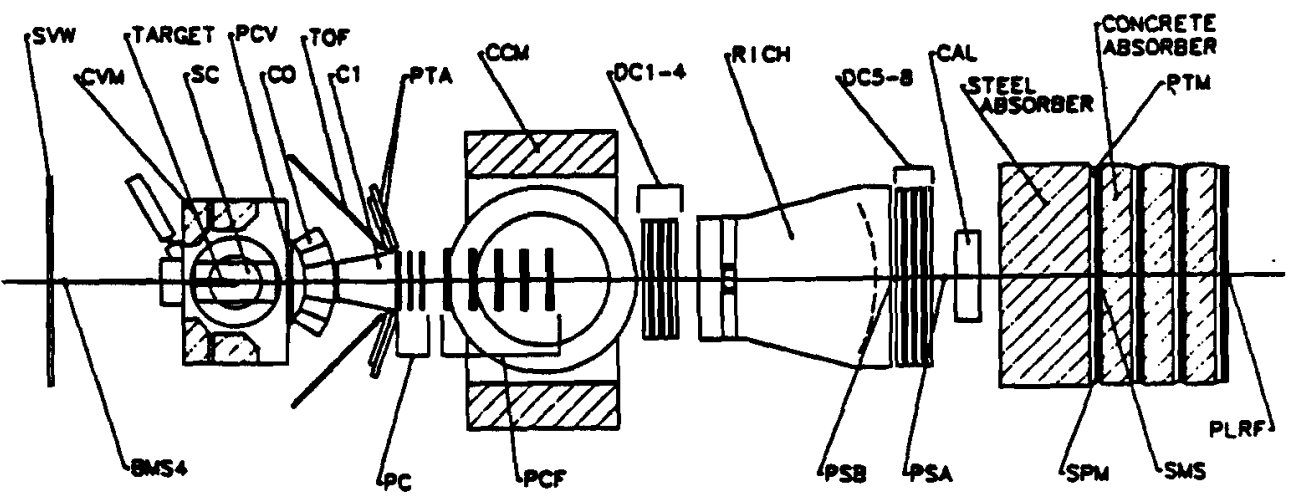

-

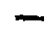

$-$
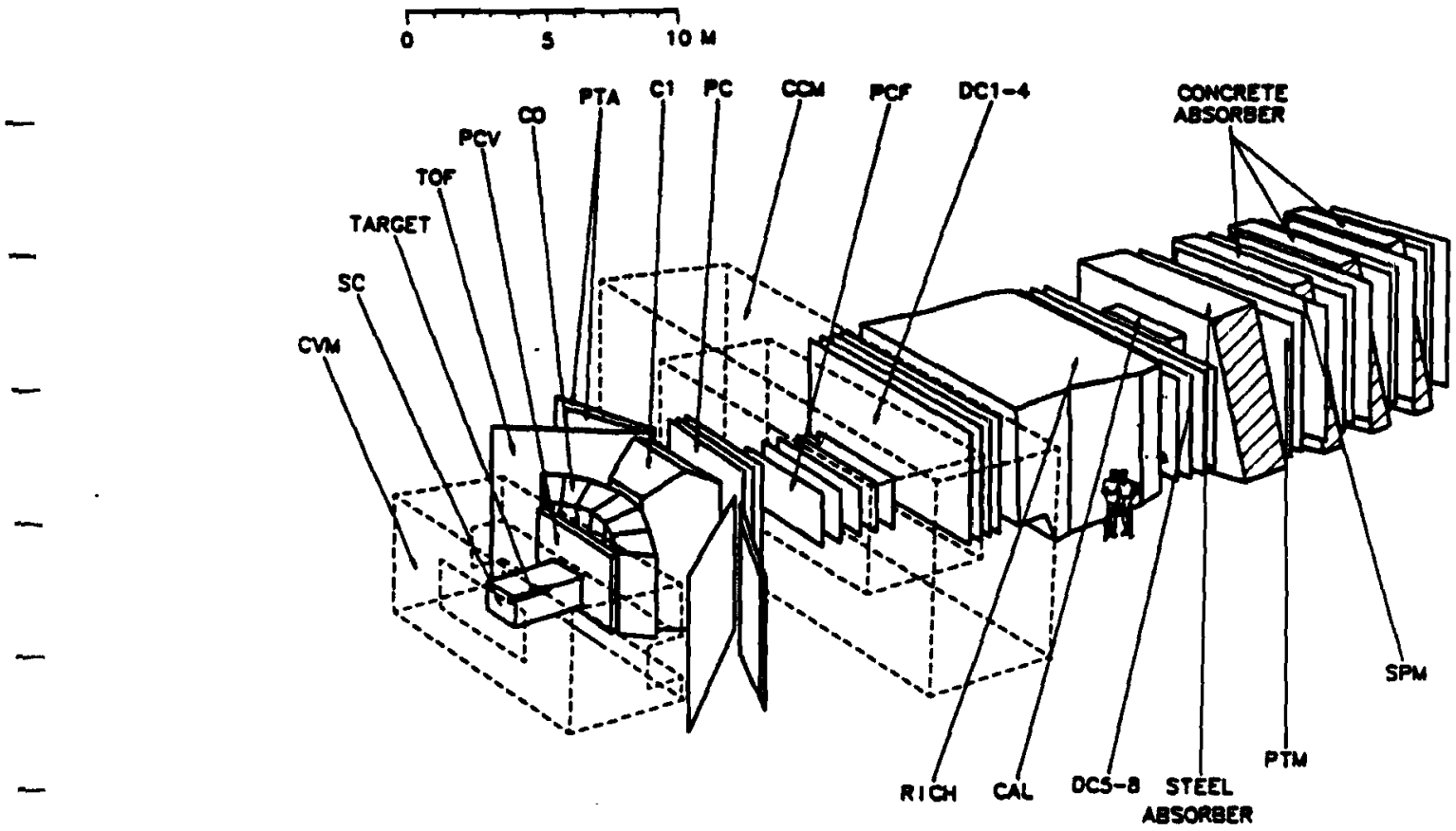

Abbildung 3.4: Der Detektor des Experimentes 665 am Fermilab 
der Datennahme. Das Xenontarget maß ca. $1.1 \mathrm{~m}$ in der Länge und $7.2 \mathrm{~cm}$ im Durchmesser. Die Dichteregulierung wurde über Druck- und Temperaturfühler kontrolliert. Der Sollwert von ca. $1500 \mathrm{kPascal}$ ergab sich aus der Bedingung, daß die Flächendichte im Xenontarget vergleichbar mit der des Wasserstofftarget sein sollte $\left(\sim 10 \mathrm{~g} / \mathrm{cm}^{2}\right)$.

\subsubsection{Die Spurnachweissysteme}

Die Elemente zum Spurennachweis der geladenen Teilchen gruppieren sich im Detektor folgendermaßen: Streamerkammer, - Proportionalkammern, - ProportionalDriftröhren, - Driftkammern, - Szintillationsfinger und -streifen.

Im Bereich um das Target und für Teilchen mit niedrigem Impuls $(\leq 5 \mathrm{GeV} / \mathrm{c}$ ) liefert die Streamerkammer die wesentlichen Informationen zur Teilchenspur und zum Wechselwirkungspunkt (Vertex). Allerdings wirken sich die Nachteile der $\sim 1 \mu \mathrm{sec}$ langen sensitiven Zeit der Kammer und der fotografischen Spurspeicherung ${ }^{9}$ und damit verbundener niedriger Triggerfrequenz (max. 8 Hertz), auf eine niedrige Rate in der Datennahme und langwährende halbautomatische Vermessung der Meßdaten aus.

Teilchenspuren, die den Vertexbereich verlassen, das Eintrittsfenster des CCM jedoch nicht treffen, werden mit Hilfe der Proportionalkammer PCV und den vier Ebenen der Proportionalzählrohre (PTA's) nachgewiesen. Die Informationen der TOF-Szintillatoren geben zusätzliche Hilfspunkte für geladene Spuren dieses Types.

Geladene Teilchen, die in das Vorwärtsspektrometer eintreten, ionisieren das Gasgemisch in den PC's und den folgenden fünf, sich im Magnetfeld befindlichen PCFProportionalkammern und induzieren einen Ladungsimpuls auf den Signaldrähten. Der weitere Verlauf einer Teilchentrajektorie wird mit den großflächigen Driftkammerpaketen DCA (sensitive Fläche $\left.2 \times 4 \mathrm{~m}^{2}\right)$ und DCB $\left(2 \times 6 \mathrm{~m}^{2}\right)$ vermessen. Im zentralen Bereich sind diese Kammern wegen der hohen Strahlintensität insensitiv. Im Bereich der DCB ist diese Zone mit den PSA-, PSB-Proportionalkammern abgedeckt. Der Aufbau dieser Kammern entspricht dem der Strahlnachweiskammern (PBT's), die eine im gesamten Bereich des Fermilab eingesetzte und damit leicht reproduzierbare Konstruktion aufweisen.

Hadronen werden in dem drei Meter dicken Eisenblock absorbiert. Nur Myonen, die sehr schwach mit Materie wechselwirken, gelangen in die Proportionalrohrebenen der PTM's. Die wesentlichen geometrischen Parameter der spurnachweisenden

\footnotetext{
${ }^{9}$ drei Kameras (mit Lichtverstärker) in Stereowinkeln von $12^{\circ}$ und $15^{\circ}$
} 
Detektoren sind in Tabelle 3.4 zusammengefaßt[38].

\begin{tabular}{|c|c|c|c|c|}
\hline \multicolumn{5}{|c|}{ Kammer Systeme } \\
\hline $\begin{array}{c}\text { Detektor } \\
\text { Name }\end{array}$ & $\begin{array}{c}\text { Detektor } \\
\text { Typ }\end{array}$ & $\begin{array}{c}\text { Sensitive } \\
\text { Fläche } \\
(\mathrm{m})\end{array}$ & $\begin{array}{c}\text { Anzahl } \\
\text { der Ebenen }\end{array}$ & $\begin{array}{c}\text { Draht } \\
\text { Abstand bzw. } \\
\text { Auflösung }\end{array}$ \\
\hline PBT & Prop. Kammer & $0.13 \times 0.13$ & $4 \times\left(\mathrm{U} ; \mathrm{Z} ; \mathrm{Y} ; \mathrm{V} ; \mathrm{Z}^{\prime} ; \mathrm{Y}^{\prime}\right)$ & $1 \mathrm{~mm}$ \\
\hline SC & Streamer Kammer & $2.0 \times 1.2 \times 0.7$ & - & $850 \mu \mathrm{m}$ \\
\hline PCV & Prop. Kammer & $2.8 \times 1.0$ & $\mathrm{Y} ; \mathrm{U} ; \mathrm{U}^{\prime} ; \mathrm{V}^{\prime} \mathrm{V}^{\prime} ; \mathrm{Y}$ & $2 \mathrm{~mm}$ \\
\hline PTA & Prop. Röhren & $2.0 \times 2.0$ & $\mathrm{Y} ; \mathrm{Z} ; \mathrm{V} ; \mathrm{U}$ & $7.2 \mathrm{~mm}$ \\
\hline PC & Prop. Kammer & $2.0 \times 2.0$ & $3 \times(\mathrm{Y} ; \mathrm{Z} ; \mathrm{V} ; \mathrm{U})$ & $3 \mathrm{~mm}$ \\
\hline PCF & Prop. Kammer & $2.0 \times 1.0$ & $5 \times(\mathrm{U} ; \mathrm{V} ; \mathrm{Z})$ & $2 \mathrm{~mm}$ \\
\hline DC1-4 & Drift Kammer & $2.0 \times 4.0$ & $4 \mathrm{Z} ; 2 \mathrm{U} ; 2 \mathrm{~V}$ & $<400 \mu \mathrm{m}$ \\
\hline DC5-8 & Drift Kammer & $2.0 \times 6.0$ & $4 \mathrm{Z} ; 2 \mathrm{U} ; 2 \mathrm{~V}$ & $<400 \mu \mathrm{m}$ \\
\hline PSA & Prop. Kammer & $0.13 \times 0.13$ & $\mathrm{Z} ; \mathrm{Y} ; \mathrm{Z}^{\prime} ; \mathrm{Y}^{\prime} ; \mathrm{U} ; \mathrm{V} ; \mathrm{U}^{\prime} ; \mathrm{V}^{\prime}$ & $1 \mathrm{~mm}$ \\
\hline PSB & Prop. Kammer & $\mathbf{0 . 1 3 \times 0 . 1 3}$ & $\mathrm{Z;Y} ; \mathrm{Z}^{\prime} ; \mathrm{Y}^{\prime}$ & $1 \mathrm{~mm}$ \\
\hline PTM & Prop. Röhren & $3.6 \times 7.2$ & $4 \times(\mathrm{Y} ; \mathrm{Z})$ & $12.7 \mathrm{~mm}$ \\
\hline \hline
\end{tabular}

Tabelle 3.4: Parameter der Spurnachweiselemente

\subsubsection{Die E665-Detektoren zur Teilchenidentifikation und das elektro- magnetische Kalorimeter}

Das Teilchenidentifikationssystem besteht aus einem Flugzeitmeßsystem (TOF) zwei Schwellenčerenkovzählern CO, C1, einem Ring abbildenden Čerenkovzähler (Ring Imaging Cherenkov) zur Bestimmung der Masse der geladenen Teilchen und einem elektromagnetischen Kalorimeter (EM) zum Nachweis von Photonen. Im weiteren Sinne wird dieses System durch die $\mu$-Identifikation mittels Hadronabsorbtion im Eisenblock ergänzt.

\section{Der Flugzeitzähler}

Das aus zwei Flügeln mit jeweils zwei Ebenen mit gegeneinander versetzten $10 \mathrm{bzw}$. $15 \mathrm{~cm}$ breiten Szintillatorstreifen bestehende System wurde bereits im Experiment NA9 der EMC am CERN benutzt. Ein Laserkalibrationssystem und ein ebenfalls für E665 neu entwickelter Startzähler verbessern die Meßeigenschaften gegenüber dem alten Aufbau. Die Zeitauflösung des Systems wurde für die Meßperiode 1987/1988 zu

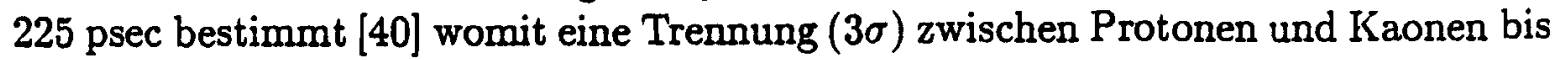


zu einem Impuls von $2 \mathrm{GeV} / \mathrm{c}$ ermöglicht wird. Abbildung 3.5 zeigt den erwarteten Verlauf von $1 / \beta$ gegen den Impuls zusammen mit am Wasserstofftarget genommenen Daten.

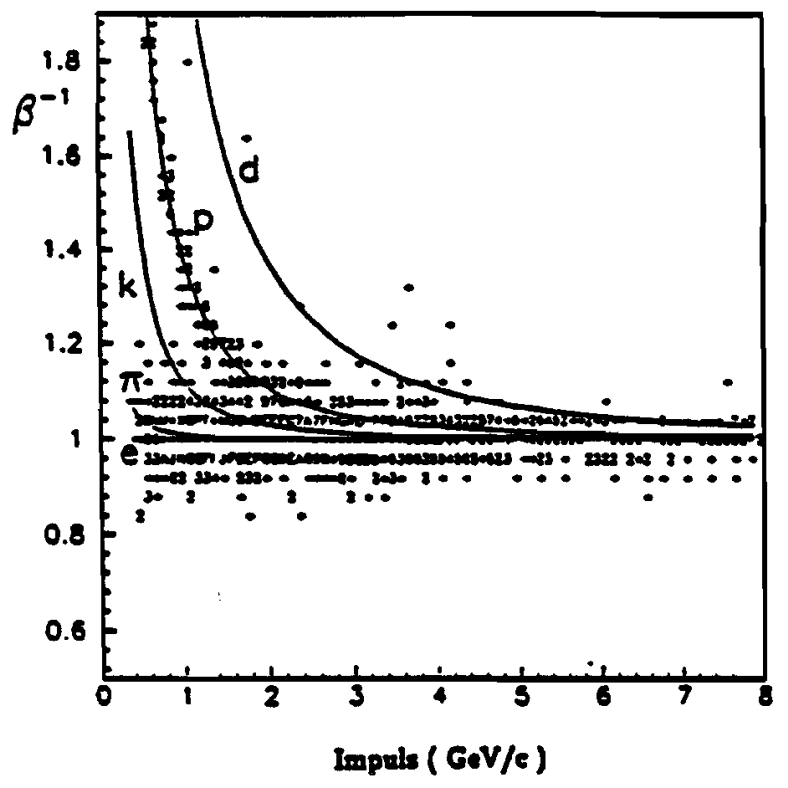

Abbildung 3.5: $1 / \beta$ gegen den Impuls für Elektronen, Pionen, Kaonen, Protonen und Deuteronen zusammen mit Vorhersagen (durchgezogene Kurven) nach [40]

\section{Der Cerenkovzähler Co}

Der Čerenkovzähler C0 ist ein multimodular aufgebauter Schwellenčerenkovzähler. Er ist mit 144 hochauflösenden Photovervielfacherröhren ausgestattet. Sein ca. 6.5 $\mathrm{m}^{3}$ großes Volumen ist mit Freon 114 ( 1,2 Dichlorotetrafluoroethan, $\mathrm{C}_{2} \mathrm{Cl}_{2} \mathrm{~F}_{4}$ ) gefüllt und wird leicht über dem Atmosphärendruck betrieben. Das Gas wurde mit Rücksicht auf die Abstimmung der Identifikationsbereiche aller Detektoren ausgewählt. Der Brechungsindex ${ }^{10}$ von 1.00141 bedingt eine Produktion von Čerenkovlicht für Pionen ab einem Schwellenimpuls von $2.6 \mathrm{GeV} / \mathrm{c}(9.3 \mathrm{GeV} / \mathrm{c}$ für liaonen, $17.6 \mathrm{GeV} / \mathrm{c}$ für Protonen). Dies ermöglicht einen Identifikationsüberlappungsbereich für das niederenergetische Ende zum TOF und für den höherenergetischen Bereich zum Čerenkovzähler C1. Gleichzeitig steht dies im Einklang mit der großen Winkelakzeptanz des Zählers ( $\pm 30^{\circ}$ vertikal, $\pm 5^{\circ}$ horizontal). Freon ist darüberhinaus als Halogen nicht brennbar und erleichtert die Einhaltung von Sicherheitsbestimmungen, die für alle Experimente am Fermilab verbindlich sind. Die sechs Mlodule sind symmetrisch aufgebaut, wobei die beiden äußeren mit jeweils zwei $2 \times 2$ Matrizen

\footnotetext{
${ }^{10}$ Eine chemische Analyse des Radiatorgases nach beendigter Datennahme ergab die folgende $\mathrm{Zu}$ sammensetzung: $93 \%$ Freon $114,5 \%$ Stickstof,,1\%Sauerstof, $1 \%$ Spurengase.
} 
von 5" Photomultiplierröhren (RCA 8854 Q) bestückt sind und die vier inneren Module mit zwei 4×4 2" Röhren (EMI 9829 QA). Jedes Modul in sich ist symmetrisch zur Mittelhorizontalen und mit jeweils zwei Hohlspiegeln ausgerüstet. Dieser Aufbau ermöglicht eine in Strahlrichtung kurze Bauweise bei optimaler Radiatorlänge und minimiertem Einfluß der Streufelder der Magneten auf die Photomultiplier. Die Module sind auf den effektiven Vertex ausgerichtet ${ }^{11}$. Diese Ausrichtung der Module unterdrückt Abbildungsverzerrungen. Der zentrale Bereich des Zählers ist durch eine lichtundurchlässige Röhre aus Tedlar-Folie, zur Unterdrückung des vom Strahl erzeugten Cerenkovlichts ausgeblendet. Die inneren 56 Röhren (im Bereich hoher Spurdichten) sind über einen modifizierten Diskriminator (LRS 4608 Z) über den pulsformenden Ausgang mit einem Time to Digital Converter (TDC LRS 2228) und alle Rōhren über einen hochimpedanten Ausgang mit einem Analog to Digital Converter (ADC LRS 2249 A) verbunden, mit dem die Pulshöhe des Signales gemessen wird. Die TDC-Information wird in der späteren Datenanalyse zur Auswahl von Röhrensignalen verwendet, die in einem ereigniskorrelierten Zeitintervall liegen.

Die sehr sensitiven (ein Photoelektron aufösenden) Röhren sind mit Mu-Metall abgeschirmt. Darüberhinaus sind die Matrixanordnungen der Röhren mit Kompensationsspulen umgeben, die die Störeinflüsse der Streufelder des CVM- und CCMMagneten weiter minimieren.

Das Gassystem des Čerenkovzählers ist ein abgeschlossenes System. Die Regelung besteht im wesentlichen aus Druckmessern, einem Kompressor, einem Verdampfer und einem gekühlten Reservoir, gefüllt mit flüssigem Freon (Siedetemperatur $3.8^{\circ} \mathrm{C}$ bei $\sim 100 \mathrm{kPa}$ ). Fällt der Außendruck ab, ist der relative Innendruck im Zähler erhöht, der Kompressor pumpt Gas aus dem Radiatorvolumen ab, komprimiert und verflüssigt es zurück ins Reservoir. Im Falle einer Außendruckerhöhung wird der Zulauf von flüssigem Freon in den Verdampfer ermöglicht und Freongas dem Volumen zugeführt. Diese Regelung ermöglicht ein Gleichgewicht zwischen dem Druck im Radiatorvolumen und dem Außendruck. Erhebliche Druckunterschiede würden die aus Mylar-Folie bestehenden Ein- und Austrittsfenster auf das Spiegelsystem drücken und eventuell die Spiegelpositionen verändern oder im Extremfall die Folien zerstören. Zur Absicherung extrem schneller Druckänderungen, die den Regelmechanismus überfordern könnten, oder bei dessen völligem Ausfall wurden zwei großvolumige Bubbler montiert. Als Flüssigkeit wurde Ổl mit geringer Viskosität und sehr niedrigem Dampfdruck gewählt. Der Öldruckpegel wurde für den Überdruckbubbler etwas höher gewählt als der des Regelbereichs der Sensoren und selbstverständlich niedriger, als das eine Beschädigung des Zählers auftreten könnte.

\footnotetext{
${ }^{11}$ Der scheinbare Vertex senkrecht von der Eintrittsebene zum Target geradlinig zurückextrapolierter Spuren erscheint näher zum Detektor als der tasächliche Vertex (der wahren, gekrümmten Spuren) [41].
} 


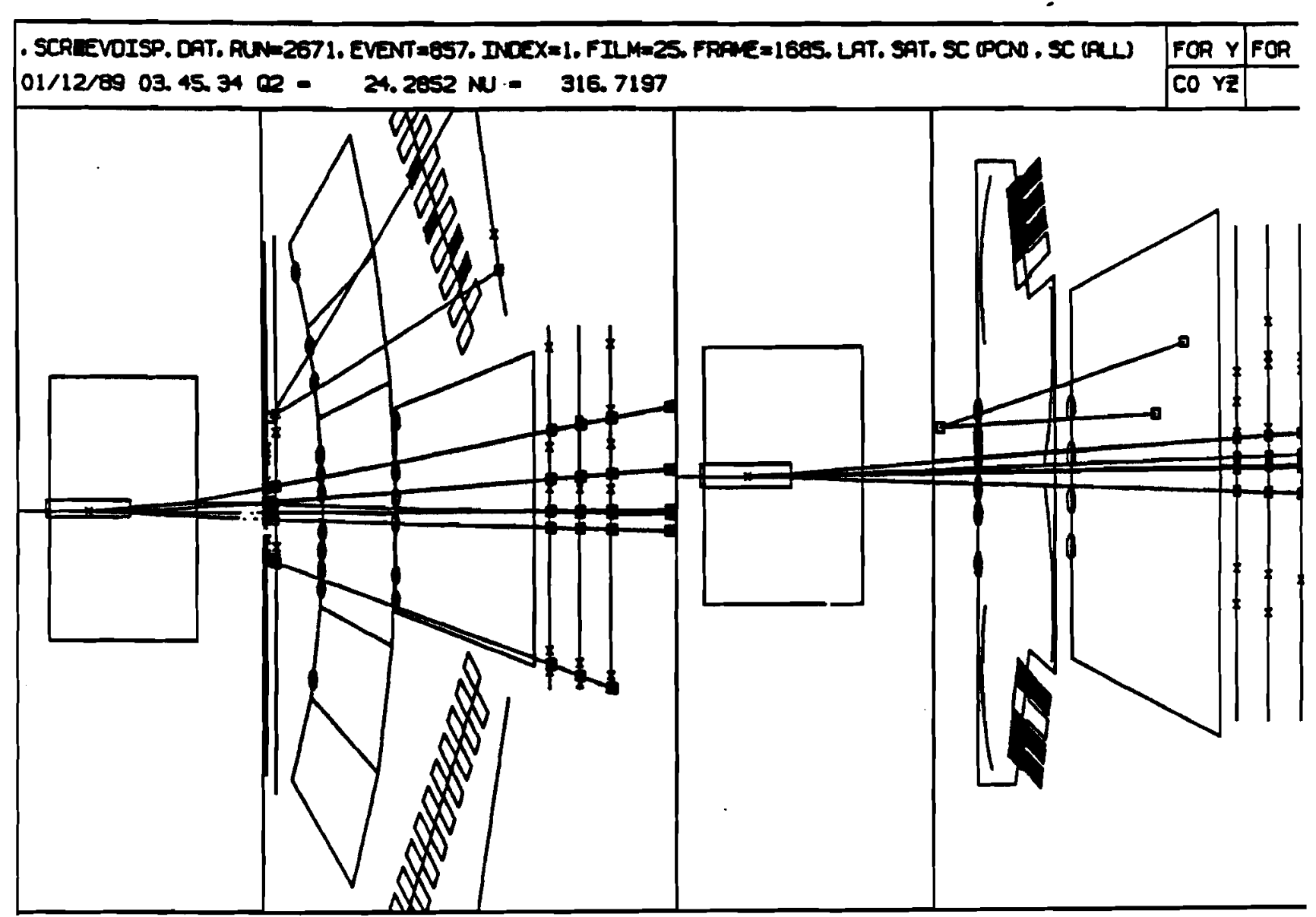

Abbildung 3.6: Tiefinelastisches Streuereignis in Auf- und Seitenansicht des Vertexbereiches 
Die mittlere Anzahl der gemessenen Photoelektronen für $\beta=1$ Teilchen beträgt ca. 15. Abb.:3.6 zeigt ein typisches tiefinelastisches Streuereignis in der Aufsicht und Seitenansicht des Vertexbereiches. Die Durchtrittsorte auf dem Eintrittsfenster entsprechen den Projektionen der vom Čerenkovlicht getroffenen Röhren. In Abbildung 3.7 ist die berechnete Anzahl von Photoelektronen gegen die Gemessene für a)

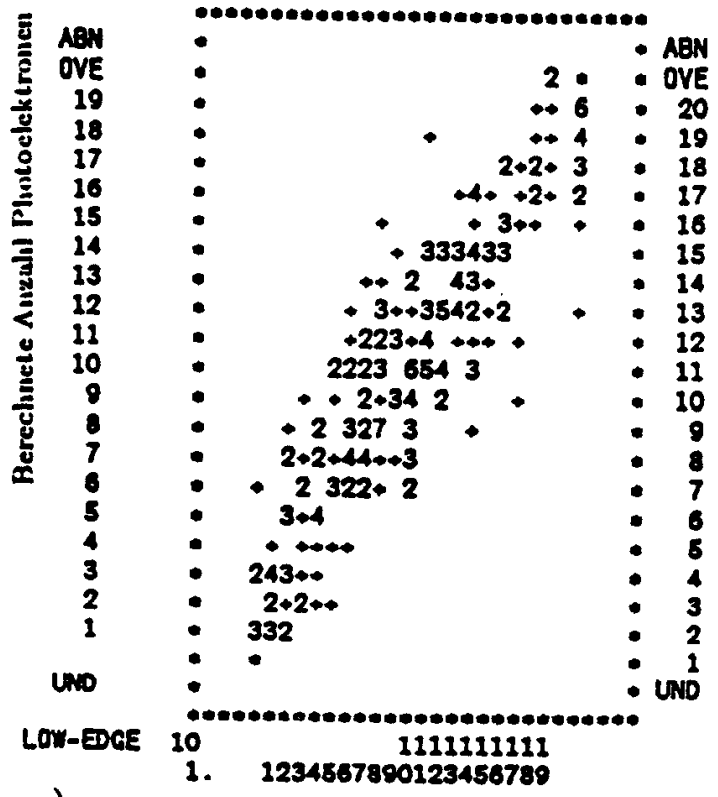

a)

Gemeasene Anzahi Photoelektronen

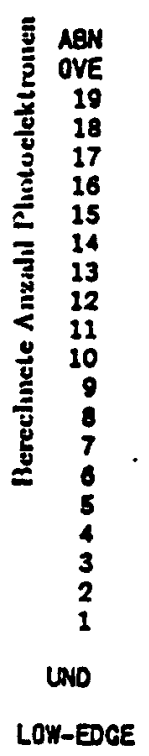

b)

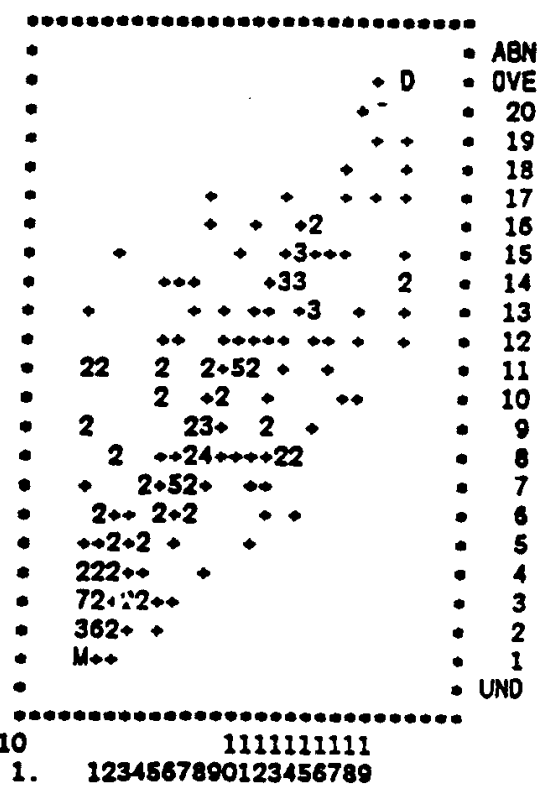

Gemessene Anzahl Photoelektronen

Abbildung 3.7: Erwartete gegen gemessene Anzahl von Photoelektronen für a) Simulation und b) Daten im Čerenkovzähler C0 [43]

Monte-Carlo-Simulation und b) Daten dargestellt. Der erwartete lineare Verliuf ist klar ersichtlich und stimmt mit der generierten Verteilung qualitativ gut übcrein.

Der Zähler ist zum gegenwärtigen Zeitpunkt in den Koordinaten des Experinentes genau vermessen[42], und die notwendige Analyse-Software in die Rekonstruktionskette eingebunden, allerdings ist der Zähler noch nicht vollständig kalibriert [43].

Der Čerenkovzähler C0 wurde von der Universität-GHS-Wuppertal in die Kiollaboration eingebracht und wird in den Bereichen Installation, Online Monitoring, Graphik und Offline-Analyse vollständig von der Wuppertaler Arbeitsgruppe betreut. 


\section{Der Čerenkovzähler C1}

Der Čerenkovzähler C1, ebenfalls ein Schwellenčerenkovzähler, ist mit einer Mischung bestehend aus 30\% Freon $112\left(\mathrm{CCl}_{2} \mathrm{~F}_{2}\right)$ und $70 \%$ Stickstoff gefüllt. Diese Gasmischung besitzt einen Brechungsindex von 1.00052. Der Schwellenimpuls für Pionen ergibt sich zu 4.3 GeV/c . Die Abbildungsgeometrie ist gegenüber dem $\mathrm{CO}$ Zähler etwas einfacher aufgebaut. Eine Spiegelmatrix von 58 Spiegeln reflektiert das Licht eindeutig auf 58 Photomultiplierröhren (RCA 8854 Q). Alle Röhren sind mit ADC's und TDC's ausgerüstet. Die mittlere Anzahl der gemessenen Photoelektronen für $\beta=1$ Teilchen wurde anhand der Elektronen-Kalibrationsmessung zu ca. 10 bestimmt.

\section{Der Ring abbildende Cerenkovzähler}

Zur Identifikation der geladenen Teilchen speziell im hochenergetischen Bereich des Impulsspektrums $(P \sim 100 \mathrm{GeV} / \mathrm{c}$ ) liefert ein Ring abbildender Cerenkovzähler (RICH) die entsprechende Information.

Das Funktionsprinzip des RICH-Detektors beruht auf seiner speziellen Geometrie (Abb.:3.8). Das produzierte Čerenkovlicht wird von den sphärischen Spiegeln mit dem Radius $r(r=9.7 \mathrm{~m}$ ) reflektiert und auf einen Photonen-Detektor im Abstand $r / 2$ fokussiert. Der Lichtkegel der Cerenkovphotonen wird hierdurch als Kreis auf einer photonenempfindlichen Kammer abgebildet. Das Čerenkovlicht tritt durch das $\mathrm{CaF}_{2}-\mathrm{Fenster}$ in den Photonen-Detektor ein. Die UV-Quanten photoionisieren Triethylamine (TEA)-Dampf. Die Photoelektronen driften in einen Proportionalkammerbereich und werden mittels der 480 Anodendrähte und 10800 Kathoden-Pads nachgewiesen [44]. Über die Bestimmung des Kreisradius wird der Öffnungswinkel des Cerenkovlichtkegels $\theta_{c}$ ermittelt. Mit $\theta_{c}=\arccos \left(\frac{1}{\beta_{n}}\right)$ und dem aus der Spurrekonstruktion ermittelten Impuls $\mathrm{P}$ ergibt $\operatorname{sich} \beta(\beta=P / E)$ und damit die Masse des Teilchens.

In diesem Photonen-Detektor wird ein Gasgemisch von 99.3\% Methan und $0.7 \%$ TEA eingesetzt. TEA zeichnet sich durch eine relativ kurze Absorptionslänge (0.5 $\mathrm{mm}$ bei Zimmertemperatur und 56 Torr Dampfdruck) aus [45].

Während der Datenperiode 1987/1988 wurde vorwiegend reines Argon als Radiatorgas verwendet. Der gemessene Brechungsindex ( $\mathrm{n}-1)$ beträgt $340 \times 10^{-6}\left(20^{\circ} \mathrm{C}\right.$, $770 \mathrm{mmHg}$ ) [46]. Die Anzahl der nachgewiesenen Photonen pro Ring betrug ca. 5.4. In Abb.:3.9 ist das Ansprechermuster eines einzelnen Beamtriggers im RICHDetektor gezeigt. Die Anodendraht- und Padstruktur ist deutlich zu erkennen. 


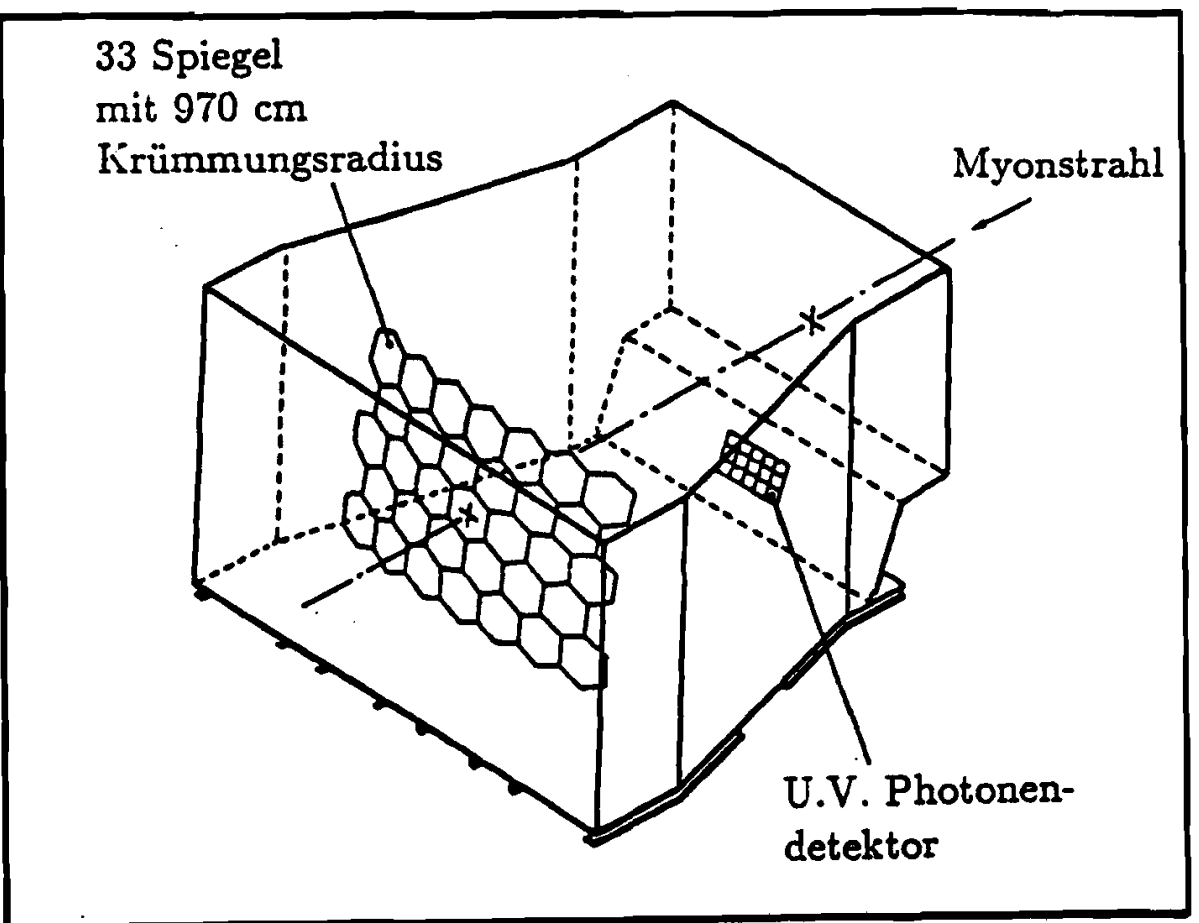

Abbildung 4.8: Der schematische Aufbau des RICH-Detektors
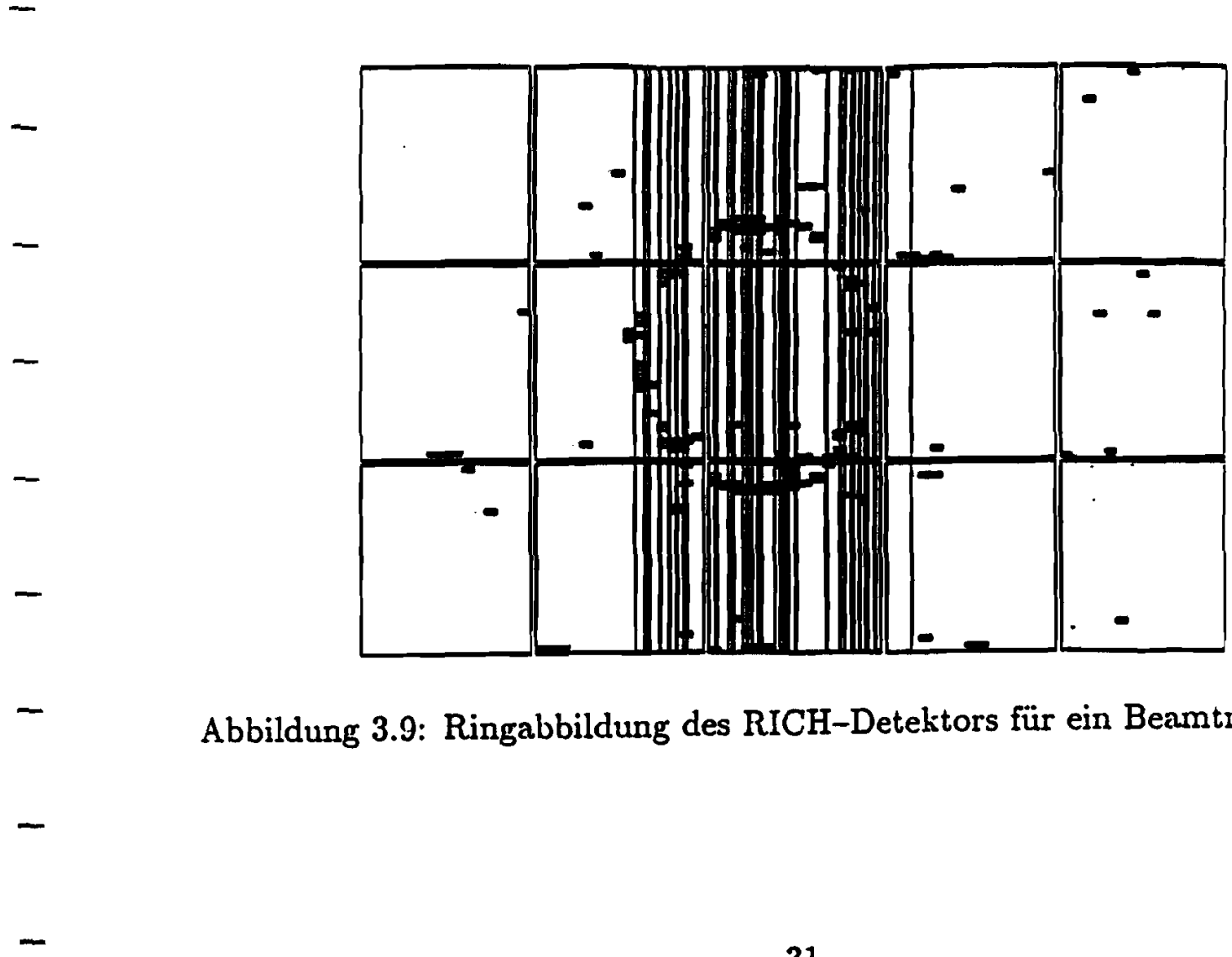

Abbildung 3.9: Ringabbildung des RICH-Detektors für ein Beamtriggersignal 
Darüberhinaus zeichnet sich ein klar erkennbarer Ring ab.

In Abb.:3.10 sind die Identifikationsbereiche der einzelnen Detektoren zusammenfassend dargestellt. Die mit 'combined' gekennzeichneten Bereiche ergeben sich bei einer Zusammenfassung der einzelnen Detektorinformationen.

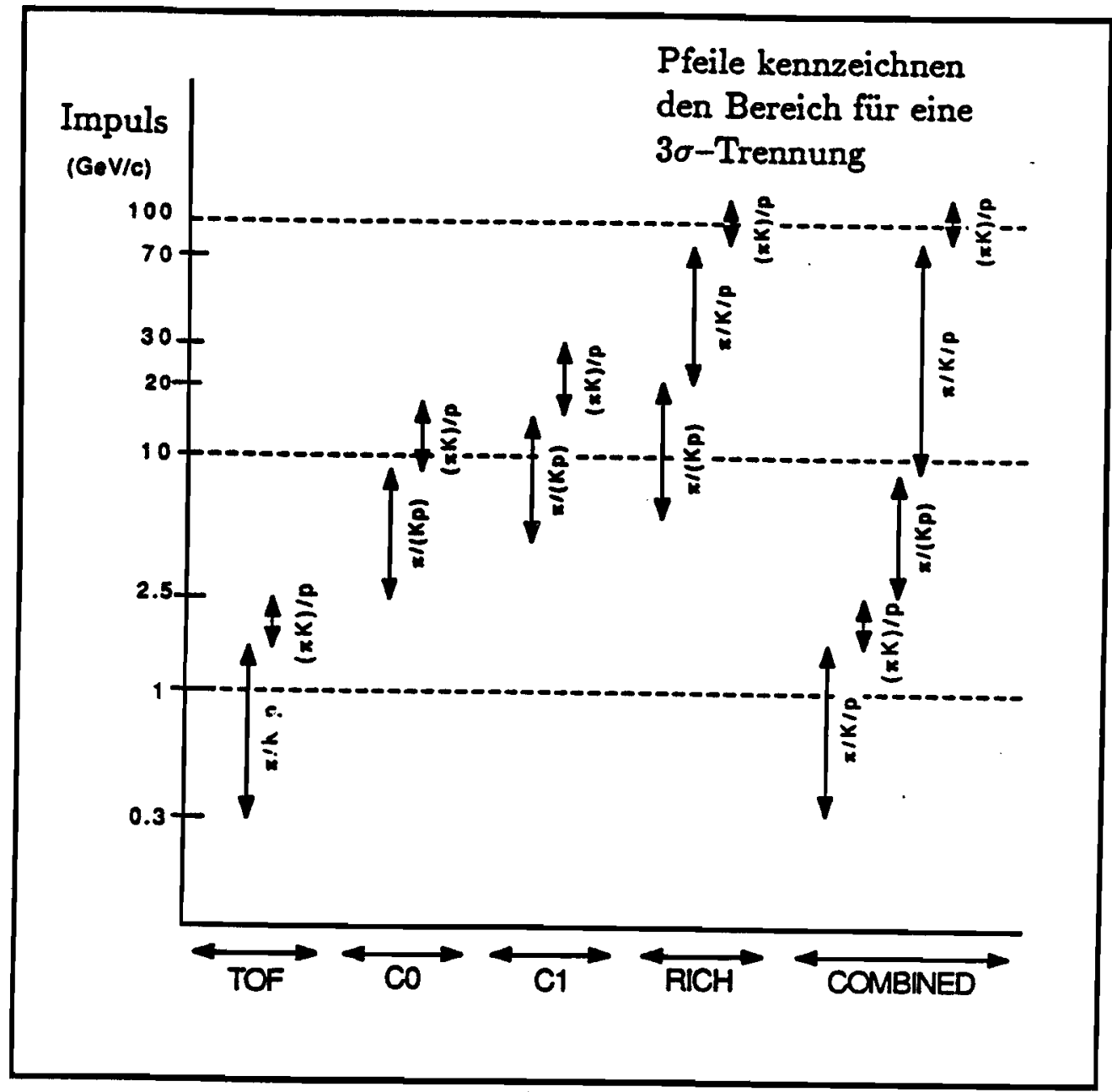

Abbildung 3.10: Identifikationsbereiche der einzelnen Detektoren

\section{Das elektromagnetische Kalorimeter}

Zur Identifikation elektrisch neutraler Teilchen und Hadronen-Elektronen-Separation wurde ein elektromagnetisches Kalorimeter (EM) konstruiert [47].

Das Kalorimeter besteht aus 20 jeweils eine Strahlungslänge tiefen $3 \mathrm{~m} \times 3 \mathrm{~m}$ sensitiven Ebenen. Jede Ebene besteht aus einer 5mm dicken Bleiplatte und einem Draht- 
kammersystem, das aus 18 Plastik-Proportionalkammern mit jeweils $161 \mathrm{~cm}$ breiten Proportional-Rohr-Zellen (Iarocci Proportional Tubes) zusammengesetzt ist. Die Drahtorientierung wechselt sich von Ebene zu Ebene orthogonal zueinander ab. Die Anodendrähte im zentralen Bereich $(1 \mathrm{~m} \times 1 \mathrm{~m})$ werden einzeln ausgelesen, die 16 Drähte der weiter außen liegenden Module zusammengefaßt.

Zu beiden Seiten des Kammersystems sind segmentierte Kathodenebenen angebracht. Die Granularität dieser Pad-Struktur nimmt von innen nach außen hin ab. Die Größe der insgesamt 1188 Pads beträgt im zentralen Bereich $4 \mathrm{~cm} \times 4 \mathrm{~cm}$, in den weiteren $50 \mathrm{~cm} 8 \mathrm{~cm} \times 8 \mathrm{~cm}$, und im äußeren Bereich $16 \mathrm{~cm} \times 16 \mathrm{~cm}$. Die Signale der Kathoden-Pads der einzelnen Ebenen werden in Strahlrichtung zusammengefaßt ausgelesen. Im Bereich des erwarteten Schauermaximums werden in vier Ebenen im inneren $1 \mathrm{~m} \times 1 \mathrm{~m}$ großen Bereich die Drähte einzeln ausgelesen, im äußeren paarweise. Die gesamte Auslese des EM erfolgt via FASTBUS. Abb.:3.11 illustriert den geometrischen Aufbau des elektromagnetischen Kalorimeters. Der Aufbau des Ka-

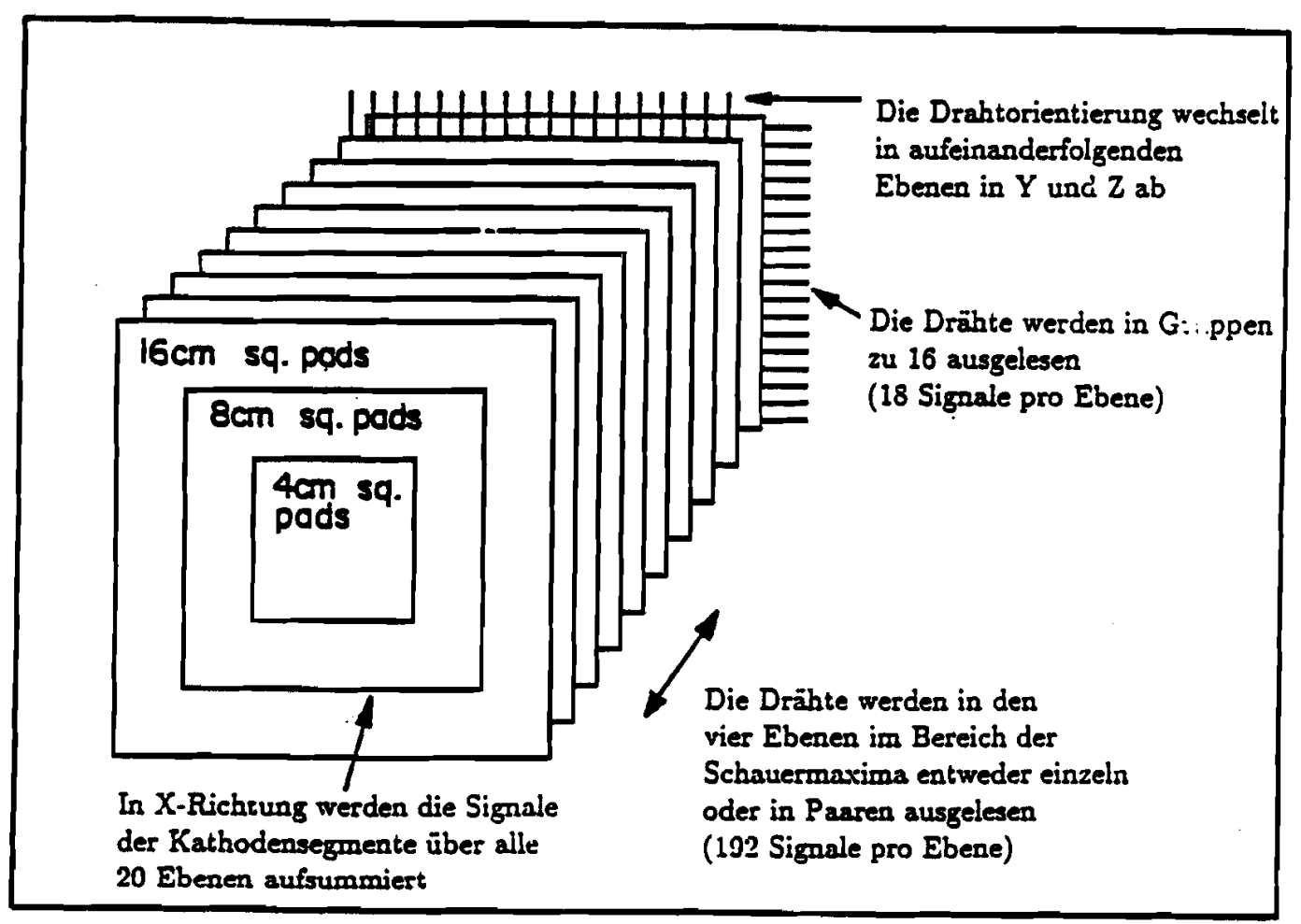

Abbildung 3.11: Der geometrische Aufbau des elektromagnetischen Kalorimeters

lorimeters gewährleistet eine Schauerrekonstruktion sowohl in logitudinaler als auch transversaler Richtung. Die Energieauflösung des EM wurde zu $\frac{\sigma_{E}}{E}=\frac{4+\%}{\sqrt{E}}+7 \%$ bestimmt [48]. 


\subsubsection{Der Trigger}

Zur Abtrennung der interessierenden tiefinelastischen Streuereignisse ist es notwendig, typische Ereignistopologien während der Datennahme zu selektieren. Die Grundidee ist, nur Ereignisse zu betrachten, in denen ein Strahlmyon im Target abgelenkt wurde.

Das LAT (large angle trigger)-Signal setzt sich zusammen aus den koinzidenten Signalen des Strahlmyons, des gestreuten Myons und einem Hochfrequenzsignal, das zur 19 nsec-'bucket'-Struktur des Tevatrons und damit zum Strahlmyon synchron ist.

Das LAT-Beamtriggersignal wird aus der Koinzidenz der sieben Signale der Fingerhodoskope (SBT's) der Strahlführung gebildet (vgl. Abschnitt:3.3). Durchdringt das Myon nicht den innersten $6 \times 6 \mathrm{~cm}^{2}$ messenden Bereich eines Hodoskopsystems (SVW, Abb.:3.4) wird das Strahltriggersignal der SBT's unterdrückt (Veto).

Zur Unterdrückung von Schauerentwicklungen im Myon-Detektorsystem ist diese Struktur in Sandwichbauweise aufgebaut (vgl. Abb.:3.4 PTM, SPM, SMS, BetonAbsorber). Anschließend an den Hadronabsorber folgt eine $7 \mathrm{~m} \times 3 \mathrm{~m}$ große Matrix bestehend aus 30 Szintillationsstreifen, die in eine untere und obere Hälfte aufgeteilt sind (SPM's). Der zentrale Bereich ist durch zwei Hodoskopebenen (SMS) aus 16 horizontal und 16 vertikal angeordneten $13.2 \mathrm{~mm}$ breiten (19.6 $\mathrm{mm}$ im äußeren Bereich) Szintillationsstreifen abgedeckt. Der Hodoskopwand folgt eine Ebene ausgestattet mit einer Doppellage von Proportionalrohrlammern (PTM's) die die Rekonstruktion der Myonspur ermöglichen. Insgesamt sind vier dieser Detektorgruppen, getrennt durch $0.9 \mathrm{~m}$ dicke Betonwände, hintereinander angebracht. Dieser Aufbau soll wie bereits erwähnt eine Schauerausbreitung, ausgelöst durch Myonen, verhindern und somit eine eindeutige Rekonstruktion des Triggerteilchens erlauben.

Das Triggersignal des gestreuten Myons wird gesetzt, sobald in mindestens drei der vier Hodoskopebenen (SPM's) ein Pulshöhensignal verzeichnet wird, allerdings nur falls kein Signal in den den Strahlbereich abdeckenden SMS-Szintillationshodoskopen vorliegt.

Liegen die Signale des Strahltriggers, des gestreuten Myons und der RF koinzident an, wird das Signal des LAT-Triggers erzeugt, der die Datenauslese initiiert.

Neben dem LAT-Trigger wird der Small Angle Trigger (SAT) im Experiment gebildet. Während der nominale minimale Streuwinkel des LAT-Triggers bei ca. $3 \mathrm{mr}$ begrenzt ist, kann der SAT-Trigger Ereignisse bis zu minimal $0.5 \mathrm{mr}$ akzeptieren. Das Triggersignal des SAT setzt sich ebenfalls aus drei zum LAT-Trigger äquivalenten 
Elementen zusammen. Zur Bildung des Strahltriggers werden zunächst die Signale der SBT-Szintillatoren mit einer Tabelle akzeptierbarer Streifenkombinationen verglichen. Ist eine Kombination erfüllt, wird das SAT-Beamsignal gesetzt.

Das zum 'gestreuten Myon' äquivalente Signal wird erzeugt, falls in den SMSHodoskopmatrixelementen, die durch die Projektion des Strahls getroffen würden, kein Signal erzeugt wird, d.h. das Strahlmyon hat auf seinem Weg mit hoher Wahrscheinlichkeit eine Wechselwirkung durchgeführt [49].

Neben dem LAT- und SAT-Trigger wurden im Experiment zusätzliche multiplizitätsabhängige Trigger für die Streamerkammer und Normalisierungstrigger für LAT und SAT gebildet. Weitere spezielle Trigger dienen zu Kalibrations- und Alignment-Zwecken sowie für spezielle systematische Studien.

Die LAT-Triggerrate, d.h. das Verhältnis der gesetzten Triggersignale zu nutzbaren Strahlteilchen (unter Berücksichtigung des Halo-Anteiles und der ApparateTotzeit), ergab sich $z u \sim 2 \times 10^{-3}$.

Schon sehr frühzeitig, während der Datennahme, wurden Studien basierend auf reinen Zählraten durchgeführt - mit Hinblick auf Konstanz und Qualität der Triggerselektivität. So ersieht man z.B. aus dem Vergleich von Messungen am vollen und am leeren Target $\left(D_{2}, 500 \mathrm{GeV}\right)$ einen $\sim 6 \%$-igen Unterschied in den Raten. Dies gibt erste Hinweise auf den Anteil von Ereignissen, in denen das Myon eine Wechselwirkung durchgeführt hat [50].

\subsection{Datennahme und Überwachung}

Das Triggersignal des Experimentes löst die Datenauslese aus. Drei 'front-end' $\mathrm{DEC}^{12}$ PDP $11 / 45$ Rechner lesen die gespeicherten Draht-, ADC-, TDC-... Information der Detektoren über CAMAC ${ }^{13}$ aus. Die Daten des elektromagnetischen Kalorimeters werden via Fastbus an den Verbindungsrechner (concatenation), eine $\mu$-Vax II (DEC) übertragen. Hier werden die Informationen der einzelnen Rechner zu einer Informationseinheit verknüpft und auf Magnetband geschrieben. Abb.:3.12 veranschaulicht den Datenfluß [51]. Pro Spill können max. 1500 Ereignisse mit einer Informationslänge von typisch $10 \mathrm{Kbytes}$ verarbeitet werden.

Die Totzeit des Apparates ist durch die $\sim 3 \mathrm{~ms}$ dauernde Auslese bestimmt und beträgt ca $10-20 \%$, abhängig von der zu verarbeitenden Information (Detektor-

\footnotetext{
${ }^{12}$ Digital Equipment Corporation

${ }^{13}$ Computer Automated Measurement And Control
} 


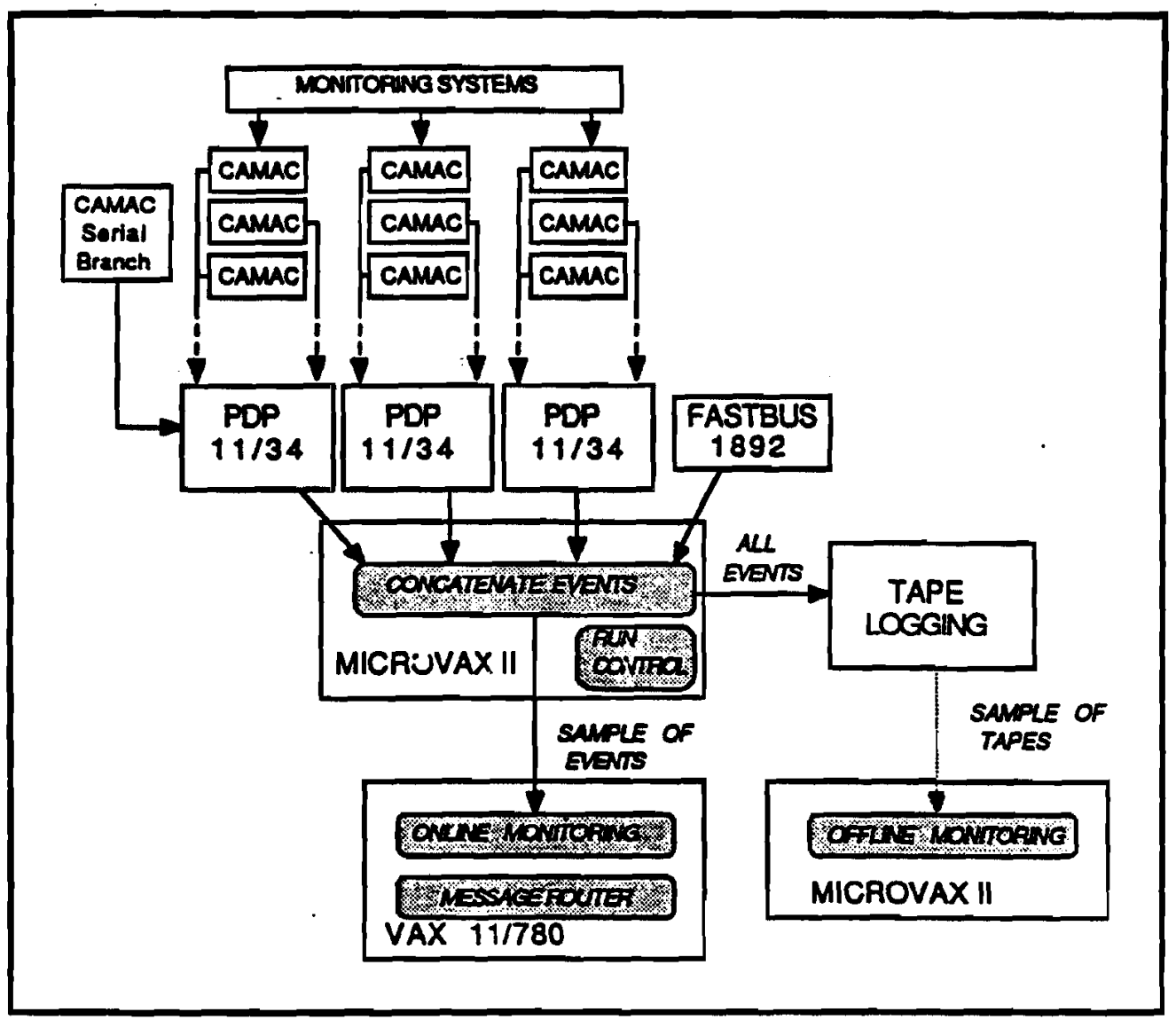

Abbildung 3.12: Datenfluß der Datenerfassung 
einflüsse) und der Strahlintensität.

Ein Teil der während des Spills aufgenommenen Daten wird in einem parallelen Datenstrom an eine Vax-11/780 (DEC) weitergeleitet und hier von detektorspezifischen Monitorprogrammen bearbeitet, so daß während der Datennahme die Qualität der Daten voruntersucht und die Funktion der Detektoren mit Daten kontrolliert werden kann.

Während der Spillpausen wurden die Nachweiselemente des Experimentes mit speziellen Monitorprogrammen getestet, die entweder auf den Front-end- Maschinen oder parallel arbeitenden Personal Computern liefen. So wurden z.B. für das TOFSystem die LASER- Kalibrierung durchgeführt, für die Cerenkovzähler C0, C1 im Radiatorvolumen befindliche LED's (light emitting diode) angesteuert und bei Proportional- und Driftkammern Pulse auf die Drähte injiziert. Nach abgelaufenem Test wurden diese Interspill-Daten über denselben Datenweg wie die Spilldaten ausgelesen, auf Magnetband geschrieben und den Monitorprogrammen auf dem VAX11/780 Rechner zugeleitet. Auf diese Weise war es möglich, nicht nur mit Daten die einwandfreie Funktion der einzelnen Detektoren zu überwachen, sondern mit speziell ausgerichteten Systemen die Datensignale definiert zu simulieren und damit den Detektor genau zu testen. Wurden Probleme festgestellt, konnten mit Diagnoseprogrammen die Fehlerursache ausfindig gemacht und je nach Schwere sofort behoben werden. Darüberhinaus bilden die auf Magnetband gespeicherten Interspilldaten die Grundlage der offline durchzuführenden Kalibration.

Parallel zu dieser Überwachungsmethode, die im allgemeinen nur eine kleine Datenstatistik erlaubt, wurde auf einem entkoppelten Rechner ( $\mu-V A X I I$ (DEC)) mit dem überwiegenden Teil der Rohdatenbänder eine 'Data validation' durchgeführt, d.h. spezielle Kenngrößen der einzelnen Detektoren histogrammiert und in $\mathrm{Zu}$ sammenhang gebracht. Ein Vergleich mit Referenzhistogrammen gab leicht Auskunft über eventuelle Abweichungen. Der Vorteil dieser zusätzlichen 'quasi'-OnlineÜberwachung bestand darin, daß die Anzahl der zu überprüfenden Detektorparameter reichhaltiger gewählt werden konnten, die Anzahl der untersuchten Ereignisse höher ausfiel und eine korrekte Abspeicherung der Daten auf den Magnetbändern kontrolliert werden konnte.

In dem Zeitraum von Oktober 1987 bis Februar '88 wurden tiefinelastische Streudaten bei Strahlenergien von $100 \mathrm{GeV}$ und $500 \mathrm{GeV}$ an flüssigem Deuterium und Wasserstoff sowie verdichtetem Xenon-Gas genommen. Darüberhinaus wurden spezielle Meßperioden eingefügt, in denen Alignment- und Kalibrationsdaten genommen wurden. Die gesamte Datenmenge wurde auf ca. 3500 High Density Magnetbänder gespeichert. Dies entspricht einer Informationsmenge von $~ 550$ Gbytes. Eine Auflistung der Meßperioden, Trigger, Targettypen und Strahlenergie ist in Tabelle 3.5 
gegeben. Die Aufbereitung und Ereignisrekonstruktion dieser Datenmenge wird im

\begin{tabular}{|l|c|ccc|c|}
\hline \multicolumn{5}{|c|}{$500 \mathrm{GeV}$ Myonen } \\
\hline \hline Target & $\begin{array}{c}\text { Myonen } \\
\times 10^{11}\end{array}$ & $\begin{array}{c}\text { LAT } \\
\begin{array}{c}\text { SAT } \\
\text { Trigger } \\
\times 10^{6}\end{array}\end{array}$ & $\begin{array}{c}\text { Daten- } \\
\text { bänder }\end{array}$ \\
\hline $\mathrm{D}_{2}$ & 2.5 & 7.5 & 5.0 & 0.21 & 1100 \\
$\mathrm{H}_{2}$ & 1.5 & 3.2 & 3.3 & 0.13 & 690 \\
$\mathrm{Xe}$ & 1.5 & 4.5 & 4.0 & 0.16 & 650 \\
\hline \multicolumn{5}{|c|}{$100 \mathrm{GeV}$} & Myonen \\
\hline \hline $\mathrm{D}_{2}$ & 0.26 & 2.6 & 1.2 & 0.016 & 340 \\
$\mathrm{Xe}$ & 0.35 & 3.5 & 1.4 & 0.044 & 440 \\
\hline
\end{tabular}

Tabelle 3.5: Datenstatistik der Meßperiode 1987/88

folgenden Kapitel erläutert. 


\section{Die Datenaufbereitung und Rekonstruktion}

Die Aufbereitung der nicht unerheblichen Rohdatenmenge kann nur mit einer wohl organisierten Software und hochleistungsfähigen Rechnern durchgefïhrt werden. In diesem Kapitel werden die konzeptionellen Grundzüge der E665-Offline-Software skizziert, die einzelnen Rekonstruktionsprogrammpakete vorgestellt, der Fluß der Datenrekonstruktion beschrieben und die benutzten Computersysteme erwähnt.

\subsection{Die Grundidee der E665-Rekonstruktionssoftware}

Die Gestaltung der Offline-Software in einem Experiment, in dem Mitarbeiter aus dreizehn internationalen Instituten zusammenarbeiten, muß dieser Randbedingung Rechnung tragen. Die Grundbedingung jedes allgemein benutzten Offline-Programmes muß daher den folgenden Kriterien genügen.

1.) Die Programme sollen weitestgehend rechnertypunabhängig (und damit transportabel) geschrieben werden. Dies hat zur Folge, daß nur eine weitverbreitete Programmiersprache benutzt wird. Die Wahl in diesem Experiment fiel auf Fortran- $77^{1}$

2.) Es muß organisatorisch möglich sein, daß gleichzeitig von mehreren Mitarbeitern unabhängig voneinander an einem Programmpaket gearbeitet werden kann. Unter diesem Gesichtspunkt wurde das am CERN entwickelte PATCHY-Programmpaket ausgewählt.

3.) Ein Memory Management System wird zur ökonomischen Datenzwischenspeicherung im Rechner-Hauptspeicher verwendet. Die Programme werden andernfalls zu speicherplatzaufwendig, und damit auf verschiedenen Rechnertypen nicht lauffähig, oder die Rechengeschwindigkeit würde erheblich reduziert. In E665 wird das ebenfalls am CERN entwickelte ZEBRA [52] Data Management Programmpaket benutzt.

Alle diese Erfordernisse werden in einem SHELL-Programm ${ }^{2}$ berücksichtigt [53]. Sämtliche Lese- und Schreibe-Operationen, die auf Dateien zugreifen, von Magnetband, Magnetbandkasseten (cartridges, $8 \mathrm{~mm}$ ) oder Speicherplatte, für alle verschiedenen Filetypen (Kontrollkarten, Konstantendateien, Rohdaten, Kalibrierungsda-

\footnotetext{
${ }^{1}$ Ein wichtiger Gesichtspunkt hierbei ist nicht nur die Eignung der Programmiersprache für den verwendeten $\mathrm{Zweck}$, sondern auch die allgemein in allen beteiligten Instituten zur Verfügung stelienden Programmbibliotheken.

${ }^{2}$ Präziser ausgedrückt handelt es sich um SHELL-Systeme auf zwei Ebenen: Zum einen im Bereich der rechnerspezifischen Betriebssysteme zur Aktivierung der Programme mit verschiedenen Optionen und zum anderen dem hier beschriebenen, in FORTRAN ausgeführten Teil.
} 
ten...), werden für den Benutzer (scheinbar) rechnertypunabhängig durchgeführt In jedem Programmpaket sind Rahmen-Standardroutinen in den wesentlichen Pro. grammphasen vorgesehen. Dieses hat den Vorteil, daß

1. ein späteres Zusammenbringen einzelner Programmpakete problemlos erfolger kann, da alle Segmente der gleichen Sequenz im Programmfluß folgen.

2. Modifikationen an I/O-Routinen (z.B. zur Anpassung an neue Massenspeicher für den Benutzer quasi unbemerkt erfolgen können.

3. neue Mitarbeiter sehr schnell, ohne lange Einarbeitungszeiten in rechnerspe. zifische Operationen, z.B. in der detektorspezifischen Software mitarbeiter können.

Eine Ausnahme von diesem Konzept stellt die in E665 entwickelte Detektorgraphik dar, die unter Verwendung des industriell entwickelten Graphikpaketes DI3000 arbeitet (vgl. z.B. Abb.:3.6). Die besonderen Anwendungsbereiche des E665-EreignisDisplays liegen im Bereich der Online-Kontrolle und der genauen Analyse kleiner Er. eignismengen. Diese beiden Anwendungsbereiche sind ohnehin zum überwiegenden Teil auf den lokalen Bereich innerhalb des Fermilab beschränkt und die Vorzüge ohn $\epsilon$ spezielle hochaufösende Farbgraphikmonitore nicht voll ausnutzbar.

\subsection{Der Datenfluß und die Rekonstruktionsprogramme}

Die Aufbereitung der Datenmenge erfordert nicht nur eine straffe Organisation der verwendeten Software, sondern auch eine logistische Reihenfolge in der technischen Abarbeitung. Eine Mischung sämtlicher Daten- und Triggertypen (Spill, Interspill, LAT, SAT ...) auf einem Rohdatenband ist im Hinblick auf die Bearbeitung unter speziellen Fragestellungen nicht sinnvoll. Interspilldaten werden im wesentlichen zur Kalibration der Detektoren verwendet, die Schwerpunkte der physikalischen Fragestellungen unterscheiden sich für Ereignisse, die mit einem Streamerkammer-Trigger oder einem LAT-Trigger genommen wurden. Diesen Ansprüchen wird im ersten Schritt der Datenaufbereitung im Split Rechnung getragen (vgl. Abb.:4.1; [54]).

Alle Rohdatenbänder werden bearbeitet und die Ereignisse nach ihrem speziellen Triggertyp getrennt und auf Magnetbänder kopiert. In einem 'side split' werden die Interspill-Daten hinsichtlich der Detektortypen aufgeteilt.

Aus Vorstudien, z.B. Triggerraten oder Auszählen der Streamerkammeraufnahmen mit und ohne Wechselwirkungspunkt, war ersichtlich, daß nur ein geringer Teil- 


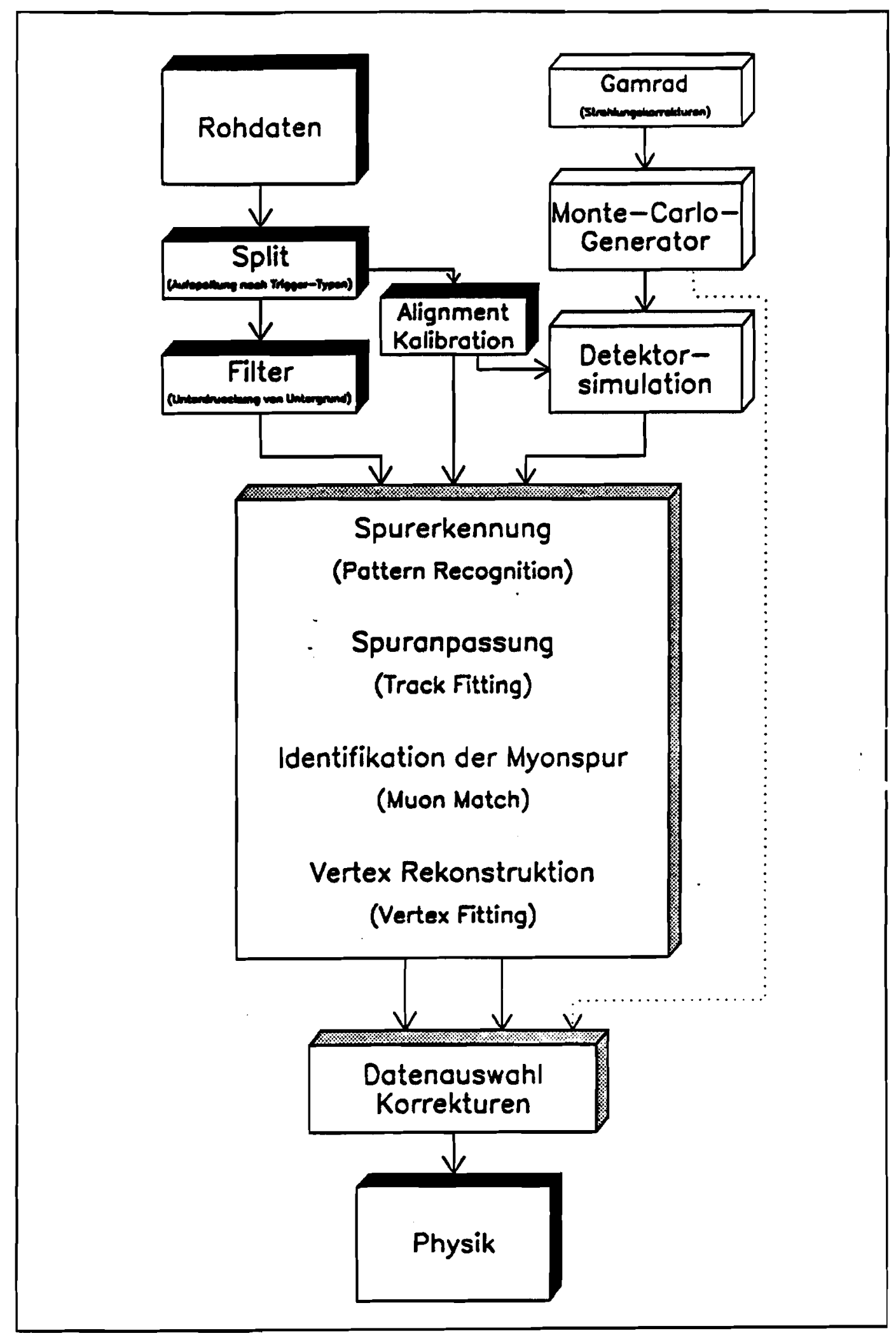

Abbildung 4.1: Datenfluß der E665-Rekonstruktions-Software-Iiette für elektronische Daten des Vorwärtsspektrometers (ohne Teilchenidentifikation). 
ca. 5-10\% - der genommenen Datenmenge interessierende tiefinelastische Ereignisse enthält. Speziell für die Programmentwicklung und Studien, die auf Rohdaten arbeiten, und mit Hinblick auf die später durchzuführende vollständige Datenrekonstruktion aller Ereignisse (data crunch) erschien es sinnvoll, offensichtlich nicht tiefinelastische Ereignisse von diesen aufgesplitteten Bändern zu eliminieren. Dies geschieht im Filter. Die angewandte Strategie beschreibt sich folgendermaßen:

1.) Es werden nur Ereignisse weiterverwertet, in denen genau eine Myon-Strahlspur rekonstruiert wurde, die zusätzlich in allen sieben Strahltriggerhodoskopen ein Signal in einem definierten Zeitfenster erzeugt hat.

2.) Falls zu dieser Spur eine einwandfreie Rekonstruktion im Vorwärtsspektrometer erfolgen kann, und aufgrund des Winkels zwischen ein- und auslaufendem Myon im Bereich des Targetortes auf ein (nicht abgelenktes) Strahlmyon geschlossen wird, wird das Ereignis verworfen, andernfalls weiterverwendet.

3.) Ein Teil aller Ereignisse wird in einer zweiten Stufe des Filters unter einem strahlunabhängigen Kriterium geprüft und für systematische Studien weiterverwendet.

Im Filterprozeß werden ca. $\frac{2}{3} \operatorname{der}(\mathrm{LAT}-)$ Ereignisse verworfen, die wesentlichste Reduktion erfolgt aufgrund des Strahlkriteriums (1.)). An dieser Stelle sei erwähnt,daß die Rekonstruktionswahrscheinlichkeit für Ein-Strahlteilchen $>99 \%$ beträgt. ZweiStrahlereignisse sind selten (ca. 10\%); die Rekonstruktionswahrscheinlichkeit ist hierfür immer noch deutlich höher als $80 \%$.

\subsubsection{Alignment und Kalibration}

Die Detektoren wurden auf Sollposition installiert und optisch vermessen (survey). Meßperioden unter speziellen Bedingungen (z.B. mit feldfreien Spektrometermagneten) wurden zur Erfassung von Alignment-Daten während der Datennahme regelmäßig eingeschoben. Die Analyse liefert präzise Ortsinformationen und erlaubt z.B. das Vermessen der Einzeldrahtpositionen und -winkel, Bestimmung der insensitiven Zonen und genaues Vermessen der Positionen der Szintillatoren. Die Auswertung der unter der Halo-Trigger-Bedingung genommenen Daten, wobei die Myonen die Detektoren flächendeckend ausleuchten, ermöglicht die Bestimmung ortsabhängiger Nachweiswahrscheinlichkeiten und Ermittlung der Orts-DriftzeitBeziehungen in den Driftkammern. Da Strahl- und Halo-Trigger während der gesamten Datennahme (untersetzt) auf Magnetband gespeichert wurden, können zeitabhängige Effekte berücksichtigt werden. 
Der speziell zur Kalibration der Teilchenidentifikationsdetektoren erzeugte $(\beta=1)$ Elektronen-Positronen-Strahl überdeckt annähernd die gesamte Akzeptanz des TOF und der Čerenkovdetektoren. Dies ist besonders vorteilhaft, da speziell in den äußeren Bereichen der Detektoren nur sehr wenig $\beta=1$-Teilchen aus tiefinelastischen Ereignissen getroffen werden, und eine Kalibration einzig mittels der Streudatensätze stark limitiert ist.

Die aus der Analyse dieser speziellen MeBperioden sowie der Analyse der InterspillDaten gewonnenen Detektorparameter werden in zeitabhängigen Konstantenfiles zusammengefaßt und definieren sowohl in der Datenrekonstruktion als auch in der MC-Simulation realistisch die Detektoreigenschaften.

\subsubsection{Das Erkennen von Teilchentrajektorien}

In der auf den Filter folgenden Stufe der Datenrekonstruktion schließt sich die Zuordnung von Drahtansprechern zu Spuren an (pattern recognition; PR). Eine detaillierte Dokumentation findet sich in [55]; an dieser Stelle wird nur ein kurzer Abriß gegeben.

Das umfangreiche Programmpaket arbeitet in vier Stufen:

1. Strahlrekonstruktion;

2. Rekonstruktion der Myonspurprojektionen in Detektoren hinter dem Eisenabsorber;

3. Rekonstruktion der Spuren im verbleibenden Bereich des Vorwärtsspektrometers;

4. Rekonstruktion der Spuren im Bereich großer Winkel.

In der ersten Stufe werden die Informationen der PBT-Kammern der Strahlmeßstation 1 bis 4 verwendet, und die gefundenen Teilchentrajektorien auf Koinzidenz mit den Signalen der getroffenen SBT-Szintillatorsignalen hin untersucht.

In der zweiten Stufe werden in den vier Ebenen der PTM's und SMS's Projektionen möglicher Myonspuren gesucht. Eine eindeutige dreidimensionale Spurzuordnung wird an dieser Stelle (noch) nicht durchgeführt (vgl. Abschnitt:4.2.4).

Die dritte Stufe wird in zwei Phasen durchlaufen: 1. in der sogenannten MyonPhase und 2. der Hadron-Phase. Diese Phasen unterscheiden sich im wesentlichen 
durch unterschiedliche Selektionskriterien, z.B. Streifenbreite, in der nach Drahtansprechern gesucht wird. In der zweiten Phase werden keine Ansprecher benutzt, die in der Myon-Phase eindeutig einer Spur zugeordnet wurden.

Innerhalb eines Durchlaufes wird im wesentlichen unabhängig von anderen Kammerpaketen zunächst, abhängig von der Geometrie, nach Spurstücken oder Raumpunkten gesucht. Anschließend werden zusammengehörige Spursegmente der einzelnen Detektoren zu Spurtrajektorien zusammengefaßt.

In der letzten Stufe des Pattern Recognition (der elektronischen Daten) werden die in den PCV gefundenen und bisher nicht verwendeten Spursegmente, unter Einbeziehung der Ortsinformation des TOF, in die PTA-Kammern projiziert und mit dort gefundenen Raumpunkten assoziiert (Spuren dieser Klasse werden in der vorliegenden Analyse nicht verwendet).

Die im PR-Programmpaket zu einer Informationseinheit zusammengefaßten Koordinaten der wahrscheinlichen Teilchentrajektorien (lines) bilden die Basis für die sich anschließende Rekonstruktionsstufe der Spuranpassung (track fitting).

\subsubsection{Die Spuranpassung}

Die Information über Position und Auflösung der Detektoren, sowie die Magnetfeldstärken im CVM und CCM, die in einem dreidimensionalen Gitter abgespeichert sind, bilden die wesentlichen Parameter für das Spuranpassungsprogramm. Auf der Basis der vom PR gefundenen Ansprecher werden zunächst lokale Fits in den einzelnen Nachweiskomponenten ausgeführt. Diese bilden die Datenstützpunkte für den globalen Fit. Die Teilchentrajektorie wird durch einen Quintic Spline Fit [56] an die Datenpunkte angepaßt, d.h. die endgültige Teilchenbahn wird durch die Parametrisierung einer Funktion fünfter Ordnung angepaßt, die ihrerseits aus mehreren Teilstücken stetig differenzierbar zusammengefaßt ist. Die Qualität des Fits wird mittels einer $\chi^{2}$ - Wahrscheinlichkeitsverteilung kontrolliert. Für den Fall realistisch angenommener Modellparameter sollte diese $\chi^{2}$-Verteilung flach sein. Abbildung 4.2 zeigt diese Verteilung für Myonspuren im Vorwärtsspektrometer (Daten).

Für Spuren, die im angereicherten Bereich kleiner $\chi^{2}$-Wahrscheinlichkeiten liegen, wird ein 'rescue' - Verfahren angewendet. Hierbei werden Drahtansprecher, die weiter als die dreifache Detektorauflösung von der Ausgleichsfunktion liegen, verworfen, und die Anpassung ohne diese Ansprecher wiederholt. 


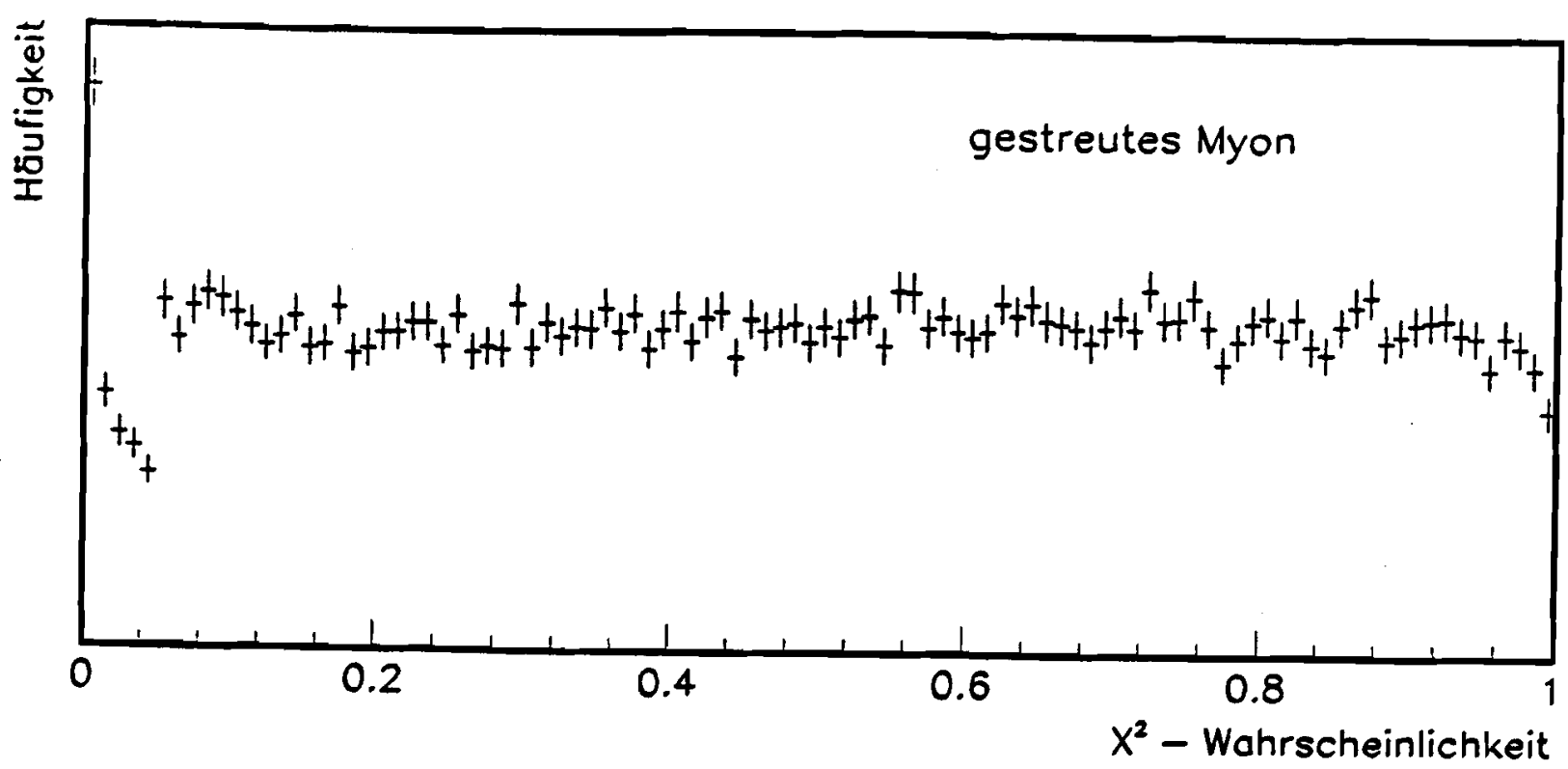

Abbildung 4.2: $\chi^{2}$-Wahrscheinlichkeitsverteilung für myonische Spuren im Vorwärtsspektrometer

Die Ergebnisgrößen des 'track fitting' sind der Impuls und das Ladungsvorzeichen, die Raumkoordinaten des am Target nächst gelegenen Fitpunltes, Spursteigungen in diesem Punkt, Fehlermatrix und Qualitätsparameter, z.B. $\chi^{2}$. Darüberhinaus werden die Spurparameter in virtuellen Nachweisebenen (key planes) gespeichert, so daß die Spurinformation in detektorlokalen Koordinatensystemen zur Verfügung stehen [57].

\subsubsection{Die Identiflkation der Myonspuren}

Dieses Programmpaket hat die Aufgabe für Spursegmente im Myon-Detektor die zugehörige Spur im vor dem Absorber befindlichen Spektrometerteil zu finden (muon match). Der Impuls der Spuren vor dem Absorber ist bekannt. Mit dieser Information kann eine Extrapolation der Teilchentrajektorie durch den Absorber unter Berücksichtigung der impulsabhängigen Vielfachstreuung durchgeführt werden. Aus allen möglichen Kombinationen der Spurstücke vor und hinter dem Absorber werden die besten Kombinationen zu durchgehenden Spuren vereinigt. 


\subsubsection{Die Vertex-Rekonstruktion}

Nachdem die Spurparameter gefunden und festgelegt sind, und die Myonspur identifiziert ist, kann der Wechselwirkungspunkt (Vertex) gesucht werden. Zunächst wird der Wechselwirkungspunkt zwischen Strahlmyon und gestreutem Myon festgelegt. Hierbei werden die Spuren unter Berücksichtigung des CVM-Magnetfeldes aufeinander zu extrapoliert. Die Orte, an denen der Raumabstand (distance of closest approach) zwischen den Spuren minimal ist, wird als Ausgangspunkt der Prozedur gewählt. In weiteren Schritten werden die hadronischen Spuren im Algorithmus aufgenommen, und alle möglichen Vertex-Kombinationen gebildet. Der endgültige Vertex ist derjenige mit dem besten $\chi^{2}$ des Optimierungsverfahrens. Nachdem der primäre Vertex bestimmt wurde, erfolgt die Suche nach sekundären Vertices, z.B. nach $V^{0}$-artigen Zerfallsorten. Spuren, deren kleinster Abstand vom $\mu \mu^{\prime}$-Vertex mehr als $5 \mathrm{~cm}$ beträgt, werden als 'close tracks' bezeichnet und nicht im endgültigen Vertexfit berücksichtigt. Dieses Kriterium ist vorläufig und von verschiedenen, die Vertexaufösung bestimmenden Faktoren abhängig. Eine Studie zur Auswahl der physikalisch zum Vertex assoziierten Spuren wird im Abschnitt 5.2 beschrieben. Eine detaillierte Programmdokumentation findet sich in [58].

\subsubsection{Das Teilchenidentifikationsprogramm}

Das Teilchenidentifikationsprogramm (Particle Identification Program, PID) befindet sich gegenwärtig noch in der Entwicklung. Die Grundidee in der Zuordnung von Teilchentyp und Spur basiert auf einer Wahrscheinlichkeitsanalyse. Jedes detektorspezifische Unterprogramm ( $\mathrm{C0}, \mathrm{C} 1, \mathrm{RICH}, \mathrm{TOF}, \mathrm{EM}$ ) berechnet für jede Spur die Wahrscheinlichkeit, daß das gemessene Signal von einem Elektron, Myon, Pion, Kaon, Proton oder Deuteron ${ }^{3}$ erzeugt wurde. Im sich anschließenden Algorithmus werden die unabhängigen Detektorwahrscheinlichkeiten zu einem gemeinsamen Satz von Wahrscheinlichkeiten abgeleitet [60]. Abbildung 4.3 illustriert den Programmfluß innerhalb dieses Programmpaketes, eine Dokumentation ist in [61] gegeben.

\subsection{Das E665-Detektorsimulationsprogramm}

Ein unerläßliches Hilfsmittel zur Bestimmung der Akzeptanz und verschiedenster Studien ist die auf der Monte-Carlo-Methode basierende Simulationsrechnung.

\footnotetext{
${ }^{3}$ Der Hypothesensatz wurde auf sechs Teilchentypen festgesetzt, da neben $e, \mu, \pi, k$ und $p$ Antideuteronen im hadronischen Endzustand in einem $\mathrm{e}^{+} \mathrm{e}^{-}-$Vernichtungs-Experiment [59] beobachtet wurden.
} 


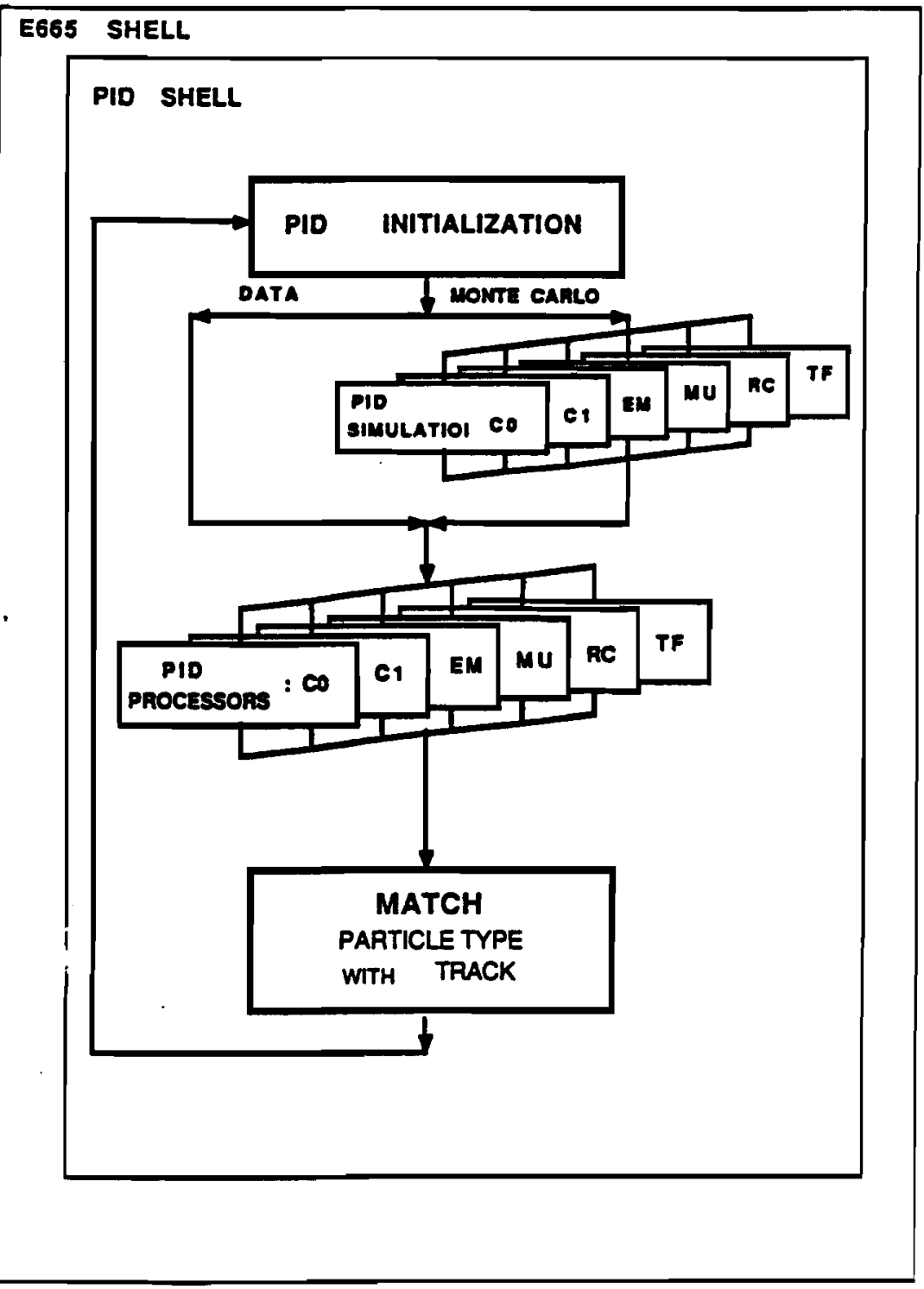

Abbildung 4.3: Programmfluß innerhalb des Particle Identification Program 
Im E665-Simulationsprogramm wird für die Erzeugung des Myonstrahles überwiegend das aus den Daten (Normalisierungstrigger: LAT-Beam, SAT-Beam) extrahierte Strahlprofil verwendet. Für Studien können Strahlprofile entweder normalverteilt oder aber über ein separates Strahlführungs-Simlulationsprogramm erzeugt und eingelesen werden.

Zur Teilchenerzeugung wird der LUND-Generator (LEPTO-Version 4.3 , JETSETVersion 6.2) benutzt. Unter Verwendung der Magnetfeldkarten werden die Spuren durch den Apparat erzeugt. Die Spurparameter werden in fiktiven, detektornahen Ebenen (key planes) zur weiteren Bearbeitung protokolliert [62]. Die ursprünglich verwendeten Modelle für Sekundärwechselwirkungen und Vielfachstreuung waren zwar für einfache Studien geeignet, modellierten aber die physikalischen Prozesse nicht befriedigend. So wurde neben dem oben skizzierten E665-Standard-MonteCarlo Stage I eine weitere Variante entwickelt. Diese verwendet im detektorspezifischen Teil das Programmpaket GEANT [63], welches Wechselwirkungen mit Materie entsprechend realistischer physikalischer Modelle berücksichtigt und eine detailliertere und zusätzlich transparentere Modellierung des Apparates erlaubt.

Im sogenannten Stage II der Monte-Carlo-Simulation erfolgt die Transformation der Parameter ins Detektorsystem und die Digitalisierung der Information. Hier gehen die geometrischen und Alignment-Konstanten und die in der Offline-Analyse bestimmten Kalibrationsparameter sowie die Detektornachweiswahrscheinlichkeiten ein. Entsprechend den aus den Daten extrahierten Stör- und Hintergrundsignalen werden Untergrundsignale modelliert. Am Ende dieser zweistufigen Simulation stehen die geometrischen Informationen und den experimentell erzeugten Detektorsignalen äquivalente simulierte Signale zur Verfügung, die anschließend in der Rekonstruktionskette identisch zu der Dateninformation bearbeitet werden [64].

\section{Rechnerkapazitäten am Fermilab}

Der Umfang der Programme ist beträchtlich. Die oben beschriebene Rekonstruktionskette basiert auf über 2500 Softwaremodulen, die innerhalb der Kollaboration entwickelt wurden und gewartet werden.

Die vollständige Rekonstruktion eines Ereignisses erfordert ca. $40 \mathrm{CPU}$-Sekunden auf einem 1 mips $^{4}$ schnellen Rechner (z.B. VAX-11/780). Die Generierung cines Ereignisses erfordert noch einmal diese Zeit.

Die Programme wurden im wesentlichen auf dem Fermilab VAX-Cluster entwickelt.

\footnotetext{
${ }^{4}$ mega instructions per second
} 
Die Datenprozessierung wurde auf einem Amdahl Rechner (5890/600 E) durchgeführt. Erste Anstrengungen wurden unternommen, die Programme auf einem $\mathrm{ACP}^{5}$-Rechner, einer Fermilab-Entwicklung, zu installieren. Abbildung $4.4 \mathrm{gibt}$ einen Eindruck der Struktur des Fermilab-Rechenzentrums.

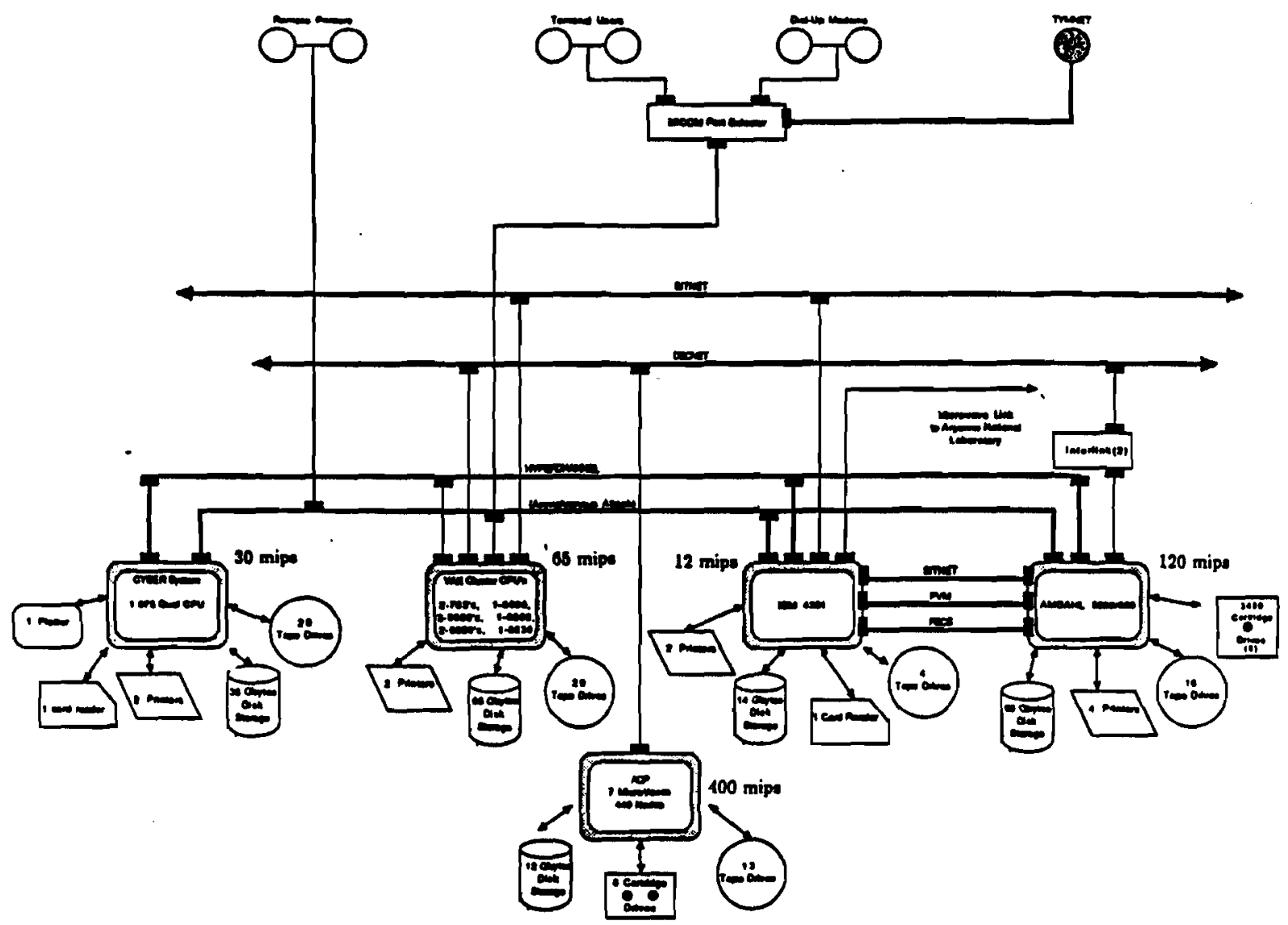

Contrat Fecllity Computing Diagrem

Abbildung 4.4: Rechnerstruktur und -leistung des Fermilab-Rechenzentrums ${ }^{6}$

${ }^{3}$ Advanced Computing Program

${ }^{6}$ Die gesamte am Fermilab zur Verfiigung stehende Rechnerleistung beträgt z.Z. ca. 1300 mips. 


\section{Die Datenanalyse}

In der vorliegenden Analyse werden die Daten verwendet, die in der Periode vom 1.-7. Dezember 1987 am Deuteriumtarget bei einer nominellen Strahlenergie von 500 $\mathrm{GeV}$ genommen wurden ${ }^{1}$. Darüberhinaus werden nur Ereignisse verwendet, die unter der Bedingung des LAT-Triggers (vgl. Abschnitt:3.4.4, $\theta_{\min } \geq 3 \mathrm{mrad}$ ) akzeptiert wurden. Ausgehend von beseits in der Filterstufe vorselektierten Ereignissen werden im folgenden Kapitel die Ereignis- und Spurauswahl diskutiert, die an die Daten angebrachten Korrekturen erläutert und die systematischen Fehler der extrahierten und korrigierten Verteilungen abgeschätzt.

\subsection{Unkorrigierte Verteilungen}

Zur Illustration sind in Abbildung 5.1 die a) $Q^{2}$, b) $Y_{B j}$ und normierten, differentiellen c) $z$ und d) $P_{t}^{2}$ Verteilungen der unkorrigierten Daten dargestellt. Die Vorhersagen des Lund-Modells (Version : Lund 4.3) sind als durchgezogene Linien eingetragen ${ }^{2}$. Die offensichtlichen Unterschiede überraschen nicht:

1. Nur ein Teil der Ereignisse stammt von der tiefinelastischen Myon-NukleonStreuung.

2. Nicht alle als Hadronspuren verwendeten Spuren sind tatsächlich hadronischen Ursprungs und stammen vom primären Vertex.

3. Apparative Effekte sind nicht berücksichtigt.

4. Rekonstruktionseinflüsse sind nicht berücksichtigt.

5. Das Monte-Carlo-Modell beschreibt die Daten nicht notwendigerweise korrekt.

Im folgenden wird die Methode dargestellt, die zu einer Minimierung bzw. Korrektur dieser Faktoren führt.

\footnotetext{
${ }^{1}$ Die Datenmenge entspricht $\sim \frac{1}{5}$ aller genommenen 500-GeV-Deuterium-Daten. Die Einschränkung wurde vorgenommen, da alle Komponenten des Apparates nach dem 1. Dezember stabil arbeiteten.

${ }^{2}$ Daten und Monte-Carlo-Verteilungen sind für den Bereich $Q^{2}>2(G e V / c)^{2}, \nu>50 G e V$ und $Y_{B j}<0.9$ dargestellt.
} 


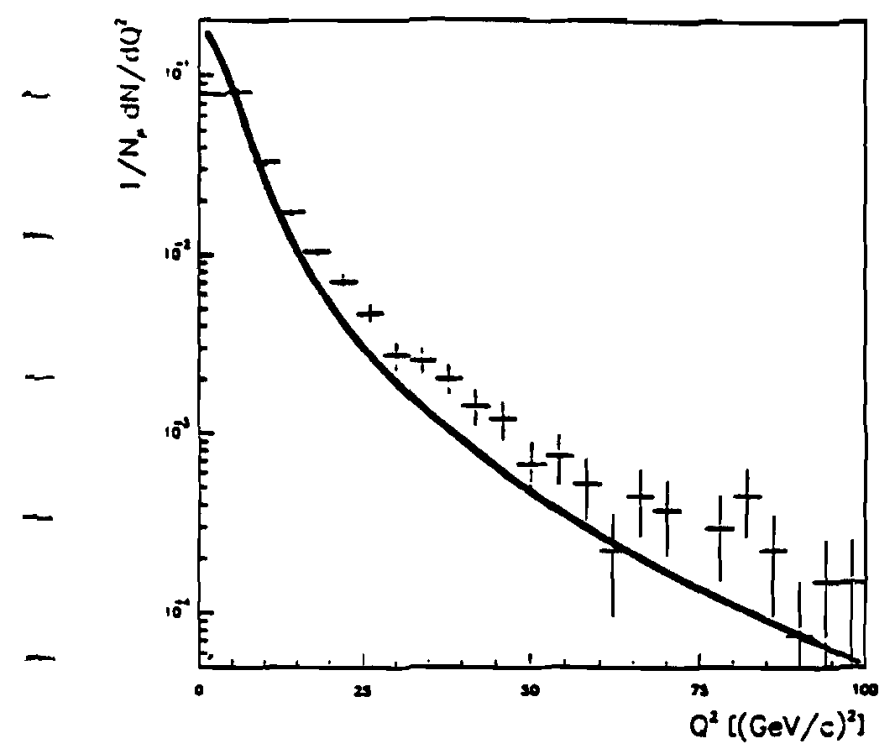

a)

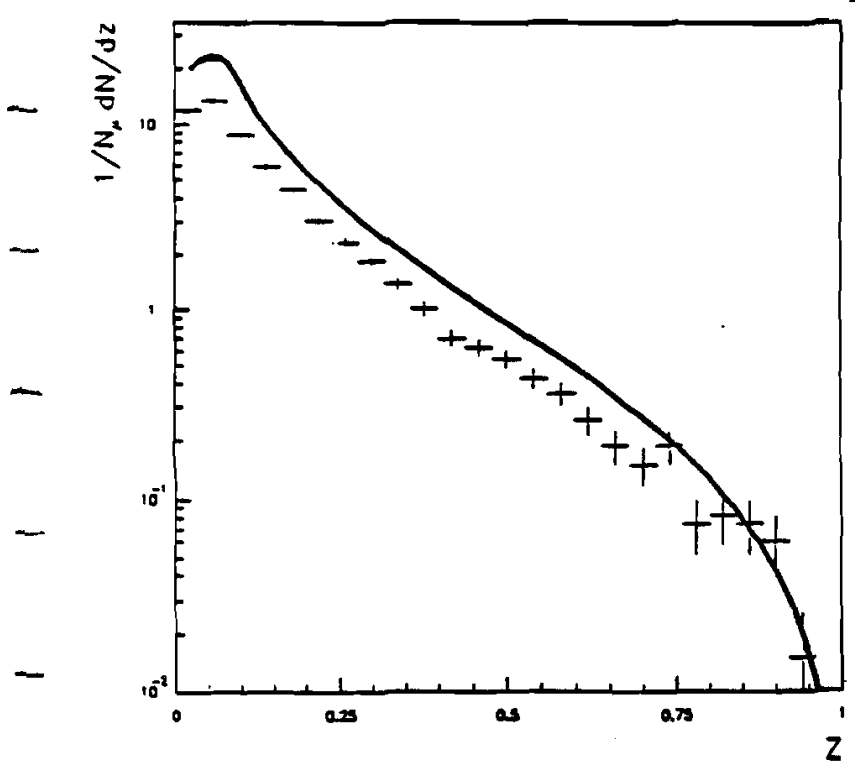

c)

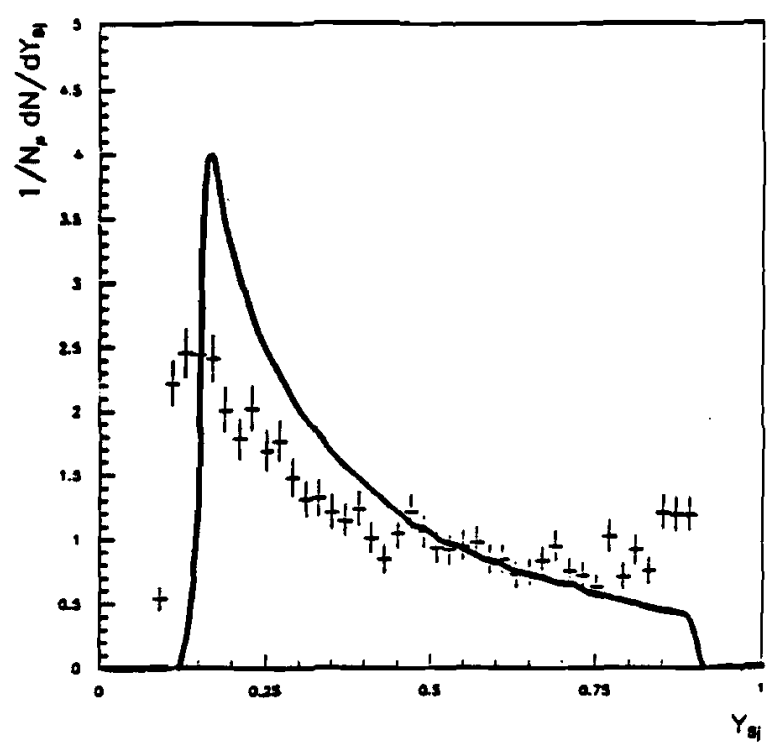

b)

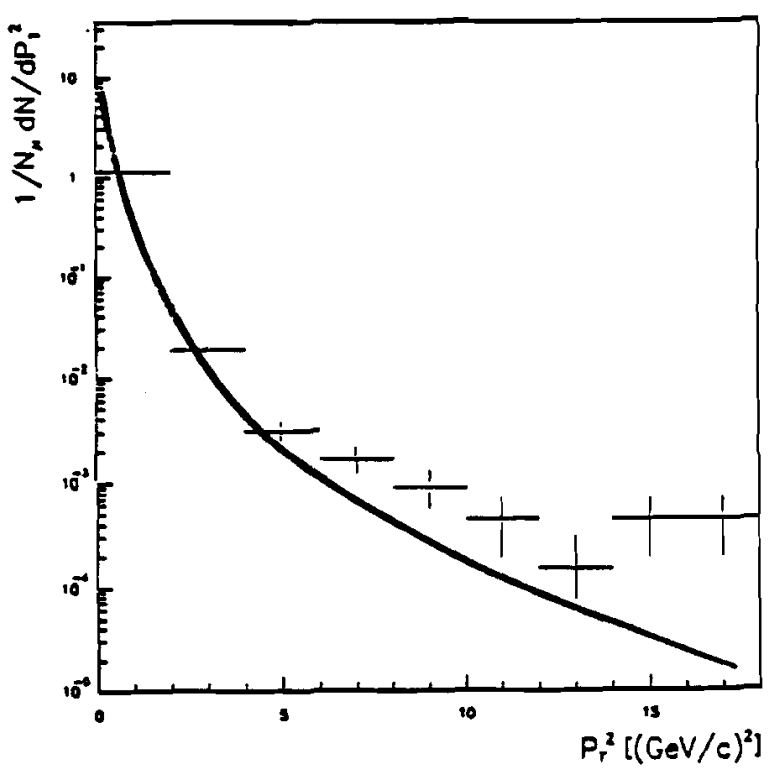

d)

- Abbildung 5.1: a) $Q^{2}$ - und b) $Y_{B j}$-Verteilung sowie die normierten, differentiellen c) z- und d) $P_{T}^{2}$-Verteilungen der 500-GeV-Deuterium-Daten (nach der Filterstufe, olne Korrekturen im Vergleich zu Monte-Carlo-Vorhersagen) 


\subsection{Die Auswahl der Ereignisse}

\section{Die Datenvorauswahl}

Schon frühzeitige Studien zur Ereignisselektion [65] haben gezeigt, daß bereits wenig einschränkende Kriterien die zu bewältigende Datenmenge erheblich reduzieren. Speziell unter dem Gesichtspunkt der Strahlungskorrekturen wurde der kinematische Bereich für $x_{B j}<0.003$, in dem die Korrekturen erheblich sind (vgl. Abschnitt:5.4.2), von der Analyse ausgeschlossen.

Gleichzeitig entfernt dieser Schnitt sämtliche Ereignisse der elastischen $\mu$-e-Streuung. Bei einem $Y_{B j}$ von 0.9 beträgt der Viererimpulsübertrag des virtuellen Photons ca. $2.5(\mathrm{GeV} / \mathrm{c})^{2}$, für die elastische $\mu$-e-Streuung jedoch nur ca. $0.45(\mathrm{GeV} / \mathrm{c})^{2}$, d.h. die elastische $\mu$-e-Streuung spielt in dieser Analyse als Konkurrenzprozeß keine Rolle.

Implizit erfordert das oben genannte Kriterium, daß zumindest ein Strahlmyon und ein Myon im Vorwärtsspektrometer gefunden wurden und ein primärer Vertex rekonstruiert werden konnte. Mit der zusätzlichen Forderung, daß der primäre Vertex innerhalb der weiten Schranken von $\pm 0.35 \mathrm{~m}$ bzgl. der Sollposition liegt, ergibt sich ein Reduktionsfaktor von ca. 10. Dies erleichtert die weitere Bearbeitung der interessierenden Ereignisse erheblich.

\section{Überprüfung der Myonrekonstruktion}

Alle hadronischen Variablen sind relativ zu den myonischen definiert. Eine sorgfältige Überprüfung der Rekonstruktion der Myonvariablen ist daher unerläßlich. Zunächst werden in der Monte-Carlo-Simulation erzeugte Ereignisse gesucht, in denen das generierte Myon nicht mit dem Rekonstruierten übereinstimmt. Nicht-Übereinstimmung bedeutet hierbei : Es gibt mindestens eine rekonstruierte 'Nicht-Myon'-Spur, deren Parameter besser mit dem Impuls und der Teilchentrajektorie des generierten Myons übereinstimmen, als das in der Rekonstruktion gefundene 'gestreute' Myon. In Abbildung 5.2 sind die kinematischen Variablen $Q^{2}$ und $Y_{B j}$ für derart untersuchte Ereignisse dargestellt. In a), b) sind die wahren, d.h. generierten Größen eingetragen (a) $0<Q^{2}<200 \mathrm{GeV}^{2} / c^{2}, \quad 0<Y_{B j}<1$, b) $0<Q^{2}<10 \mathrm{GeV}^{2} / c^{2}, \quad 0.6<Y_{B j}<1$ ), in c) und d) $Q^{2}$ und $Y_{B j}$ aus der Rekonstruktion. Es ist deutlich erkennbar, daß im Bereich kleiner (wahrer) $Q^{2}$ die rekonstruierten Ereignisvariablen zu großen $Y_{B j}$ und höheren $Q^{2}$ verschoben werden. Der gesamte Anteil der falsch rekonstruierten Ereignisse beträgt zwar nur ca. $1 \%$, in eingeschränkten kinematischen Bereichen kann der relative Anteil jedoch erheblich werden. So sind z.B. im Bereich $W^{2}>750$ 
$\mathrm{GcV} 20 \%$ aller Myonen falsch zugeordnet. Eine Untersuchung der Parameter dieser nicht korrekt rekonstruierten Myonen, wie z.B. Impuls, Durchtrittsorte im Detektor, Vertexposition, Abstand zum Vertex, $\chi^{2}$ der Spuranpassung ..., ließen sich nicht eindeutig auf nicht zu $Q^{2}, Y_{B j}$ unkorrelierte Variablen zurückführen. Da ein Schnitt zu hohen $Y_{B j}$ zur Unterdrückung von Bremsstrahlungsereignissen ohnehin notwendig ist, und darüberhinaus die Triggerakzeptanz des Detektors zu kleinen $Q^{2}$ hin abfallt, wurde der Bereich $Y_{B j}>0.85, Q^{2}<2(G e V / c)^{2}$ von der Analyse ausgeschlossen.
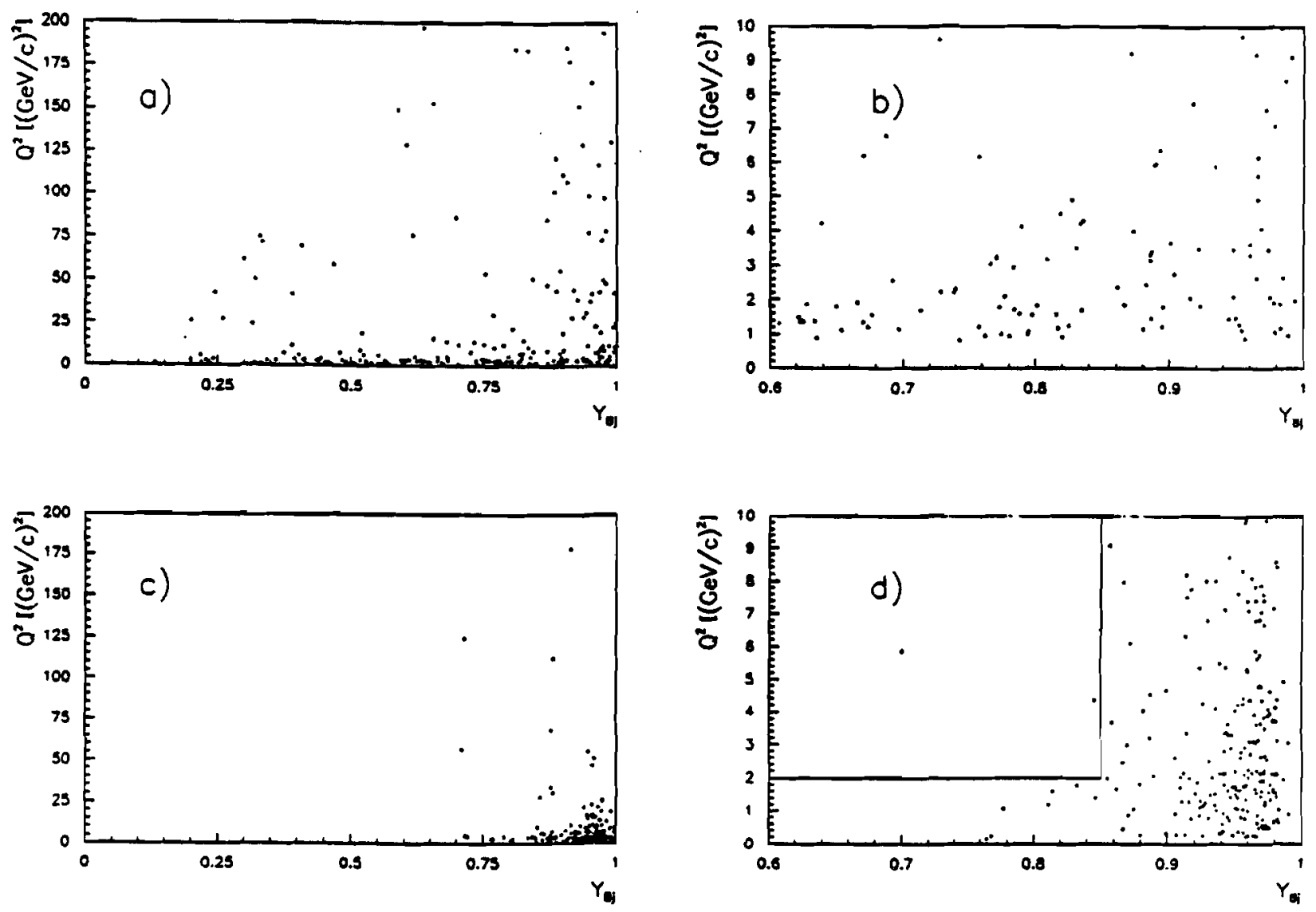

Abbildung 5.2: $Q^{2}$ vs. $Y_{B j}$ a) und b) generierte Größen c), d) rekonstruierte Größen für inkorrekt rekonstruierte Myonen. (a),c): $0<Q^{2}<200 \mathrm{GeV}^{2} / \mathrm{c}^{2}, \quad 0<\mathrm{Y}_{B},<1$, b),d): $\left.0<Q^{2}<10 \mathrm{GeV}^{2} / \mathrm{c}^{2}, 0.6<Y_{B j}<1\right)$

Diese Limitierung reduziert die inkorrekt rekonstruierten Myonen um einen Faktor 20. Resteinflüsse dieses Rekonstruktionseffektes werden nach Anbringen dieses Auswahlkriteriums als vernachlässigbar angenommen. 
Einschränkung des kinematischen Bereiches unter Berücksichtigung der Spektrometeraufösung

Der kinematische Bereich wird weiterhin zu kleinen $\nu(\nu>100 \mathrm{GeV})$ und kleinen $W^{2}$ $\left(W^{2}>100(\mathrm{GeV} / \mathrm{c})^{2}\right)$ eingeschränkt. Die Impulsauflösung des Detektors hängt im wesentlichen von der Anzahl und Geometrie, sowie der Positionierung der spurnachweisenden Elemente (bei gegebenem $\int B d l$ ) ab. Für hohe Energien des gestreuten Myons wird die Differenz $E_{0}-E_{\mu^{\prime}}$ klein und der Fehler in $\nu$ divergiert. So ergibt sich z.B. bei $\nu=100 \mathrm{GeV}$ und der mittleren Strahlenergie von $490 \mathrm{GeV}$ ein $\delta \nu / \nu$ von $\sim 8 \%^{3}$.

Die bisher abgeleiteten Selektionskriterien wirken sich nicht nur unter den oben genannten Gesichtspunkten aus, sondern bewirken darüberhinaus folgende Effekte:

1. Bereiche niedriger Akzeptanz - neben der Rekonstruktionswahrscheinlichkeit dominiert die geometrische Akzeptanz des LAT-Triggers - bei kleinen $Q^{2}$ und $\nu$ werden unterdrückt.

2. Die Bedingung $Y_{B j}<0.85$ ergibt, daß der minimale Impuls des gestreuten Myons zu $70 \mathrm{GeV} / \mathrm{c}$ limitiert ist (für $E_{0}=490 \mathrm{GeV}$ ), und damit praktisch keine Myonen aus Pionzerfällen einen Trigger in den Hodoskopen vortäuschen können.

3. Die Vielfachstreuung der Myonen wird im Eisenabsorber (aufgrund des minimalen $\mu$-Impulses von $70 \mathrm{GeV}$ ) klein gehalten.

4. Die hohen kohärenten Anteile der Strahlungskorrekturen werden stark unterdrückt.

5. Der Schnitt zu kleinen $W^{2}$ trennt elastische Streuereignisse sowie Nukleonenresonanzen vollständig ab.

Zur Illustration der Einflüsse der kinematischen Schnitte ist in Abbildung 5.3 das Verhältnis aus Monte-Carlo-generierten-Ereignissen zu Rekonstruierten in Abhängigkeit von $W^{2}$ dargestellt. In a) vor und in b) nach Anbringen der Selektionskriterien.

\footnotetext{
${ }^{3}$ Die mittlere Impulsauflösung für die Strahlmyonen beträgt $\sim 0.5 \%$. Die Impulsauflösung des Vorwärtsspektrometers $\delta P / P=P * 5 * 10^{-5}$
} 


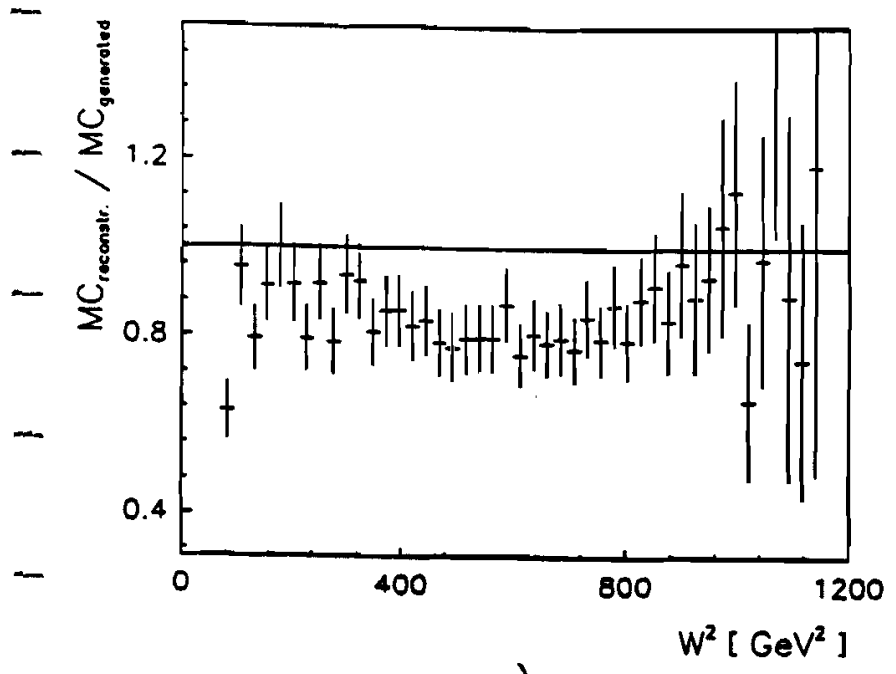

o)

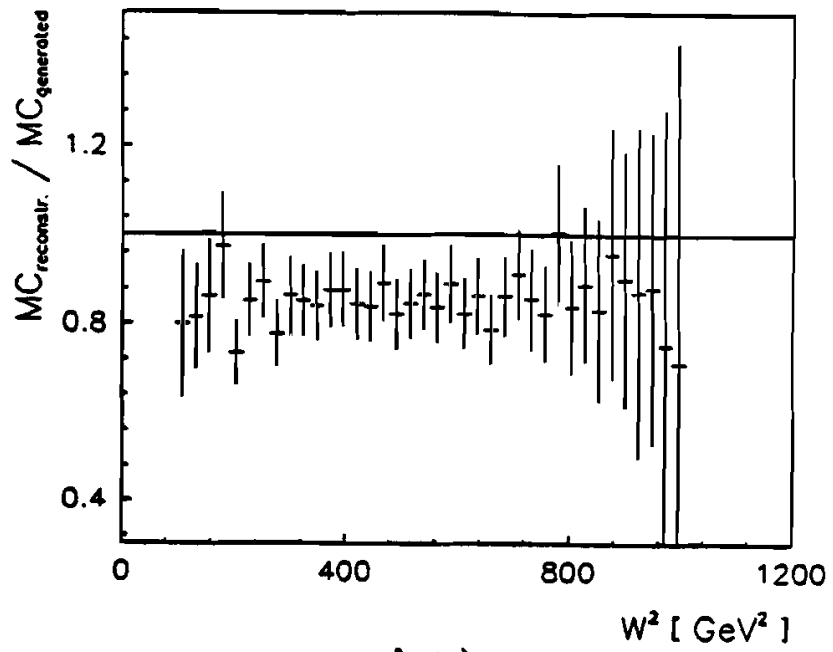

b)

Abbildung 5.3: $W^{2}$-Akzeptanz a) vor und b) nach der Ereignisauswahl

\section{Auswahl der Vertices}

Selbstverständlich sollen nur Ereignisse betrachtet werden, die am Deuterium gestreut wurden. Abbilciung 5.4 a) zeigt die Vertexortverteilung in Strahlrichtung im Bereich -17m- -4m. Deutlich sind die Veto-wall $(x \sim-16 \mathrm{~m})$, das Target $(\mathrm{x} \sim-12 \mathrm{~m}-$ $-10 \mathrm{~m})$, die PCV-Kammern $(\mathrm{x} \sim-8.6 \mathrm{~m})$ und der $\mathrm{C} 1(\mathrm{x} \sim-7 \mathrm{~m}--5.3 \mathrm{~m})$ zu erkennen. In Abbildung $5.4 \mathrm{~b}$ ) ist die Vertexposition im Bereich $-12 \mathrm{~m}--10.2 \mathrm{~m}$ hervorgehoben. Der endgültig verwendete Bereich ist durch die Markierungen hervorgehoben.

Die Aufiösung der Rekonstruktion des primären Vertex beträgt ${ }^{4} \sigma_{x} \sim 2 \mathrm{~cm}\left(\sigma_{y, z}<\right.$ $0.2 \mathrm{~mm}$ ). Ein nahezu vollständiger Ausschluß der Streuereignisse am Targetmantel $(x-3 \sigma)$ zur Vermeidung A-abhängiger Effekte würde zu einer erheblichen Redultion des Datensatzes führen. $\mathrm{Da}$ die A-Abhängigkeit des hadronischen Endzustandes im verwendeten kinematischen Bereich klein ist [66], wurde auf eine derartige Einschränkung zugunsten der höheren Datenstatistik verzichtet.

Darüberhinaus werden nur Ereignisse verwendet, in denen ein primärer Vertex mit genau einem einlaufenden und mindestens einem auslaufenden Myon, unabhängig von der Anzahl der Hadronen, gefunden wurde.

\footnotetext{
${ }^{4}$ Nach Anbringen der in Tabelle 5.1 aufgeführten Auswahlkriterien
} 

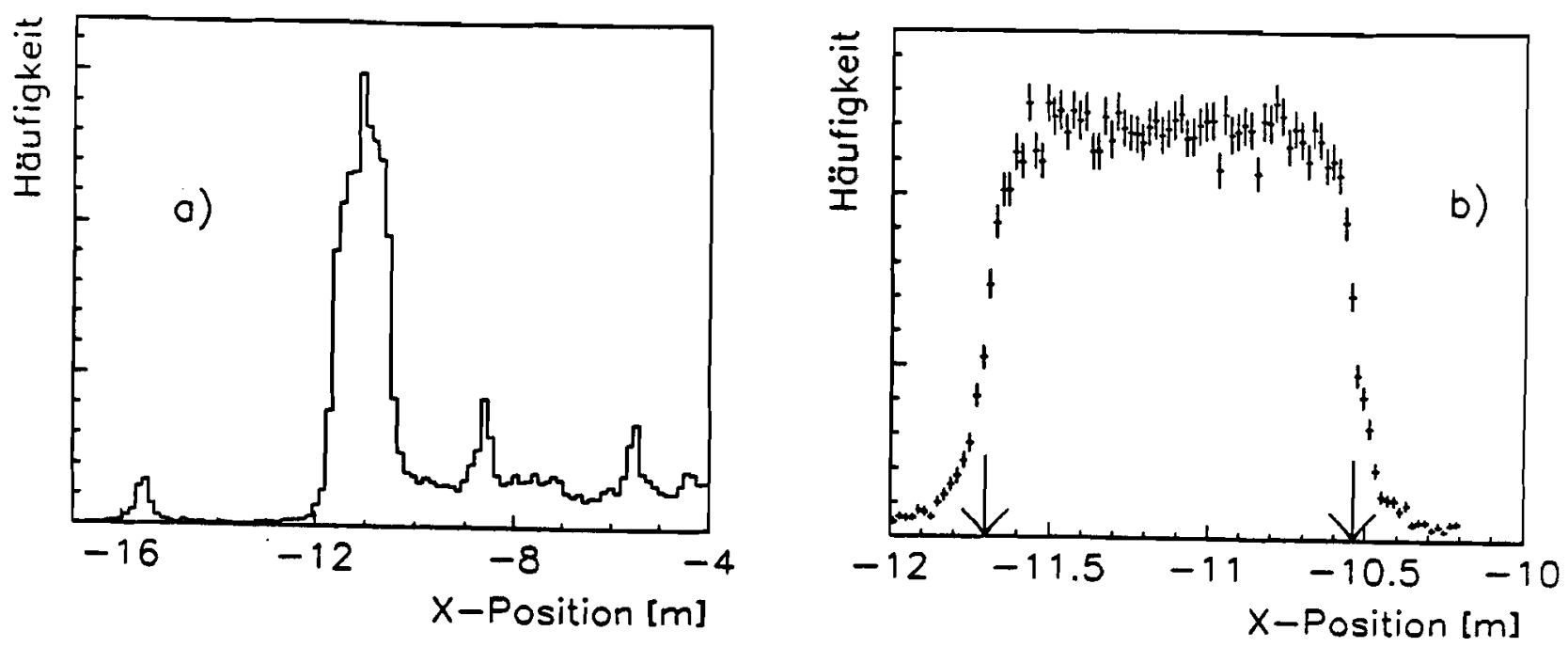

Abbildung 5.4: Position der primären Vertices in Strahlrichtung im Intervall a) $-17 \mathrm{~m}$ $--4 m$ und b) $-12 m--10 m$

\section{Qualitätsschnitte}

Einige zusätzlich angebrachte Schnitte zur Definition des Strahlphasenraumes, Qualitätskontrolle der Spurparameter des gestreuten Myons und des Vert=xfit: reduzi : en den Datensatz nur unerheblich, können aber zu sehr schwer zu korrelierenden (eben wegen der geringen Häufigkeit) Verschmierungen der hadronischen Variablen führen.

Sämtliche angebrachten Schnitte sind in Tabelle 5.1 aufgelistet.

Parallel zu den oben besprochenen Auswahlkriterien ist ein Satz weniger einschränkencler Bedingungen in Spalte 4 (Studie) verzeichnet. Die gesamte Analyse wurde mit diesen zwei Sätzen von Schnitten zur Abschätzung der systematischen Unsicherheiten durchgefuhrt.

Im weiteren werden in der Analyse nur Ereignisse betrachtet, die die stärker einschränkenden Bedingungen erfüllen, anderenfalls wird darauf hingewiesen.

Zum Abschluß dieses Abschnittes ist in Tabelle 5.2 die Reduktion des Datensatzes ausgehend von der Anzahl der Strablmyonen aufgelistet.

Die entsprechende Anzahl der zur Akzeptanzkorrektur zur Verfügung stehenden voll

\footnotetext{
${ }^{5}$ Degrees of freedom: Anzahl der Freiheitsgrade in der Spuranpassung

${ }^{6}$ Distance of closest approach to vertex

${ }^{7}$ Distance of closest approach over error
} 


\begin{tabular}{|c|c|c|c|c|c|c|c|}
\hline Klasse & Variable & \multicolumn{3}{|c|}{ Intervall } & \multicolumn{3}{|c|}{ ( Studie) } \\
\hline \multirow[t]{4}{*}{ Strahlmyon } & Impuls P $[\mathrm{GeV} / \mathrm{c}]$ & 300. & $<\mathrm{P}<$ & 650. & - & 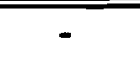 & \\
\hline & Divergenz $\mathrm{Y}^{\prime}[\mathrm{mr}]$ & -2. & $<Y^{\prime}<$ & 1. & - & - & \\
\hline & Divergenz Z' [mr] & -1 & $<Z^{\prime}<$ & 1. & $\overline{-}$ & - & \\
\hline & $\chi^{2} / D O F^{6}$ & & $\chi^{2} / D O F<$ & 2.5 & - & - & \\
\hline \multirow[t]{6}{*}{ Myon } & Spuranfang & & vor $\mathrm{CCM}$ & & & $\overline{-}$ & \\
\hline & Spurende & & in DC's & & & - & \\
\hline & DOF & & $\geq 5$ & & & - & \\
\hline & $\mathrm{DIS}^{6}[\mathrm{~mm}]$ & & $\overline{\leq 3}$ & & & - & \\
\hline & $\mathrm{DOE}^{7}$ & & $\leq 4$ & & & - & \\
\hline & $\chi^{2} / D O F$ & & $<5$ & & & - & \\
\hline \multirow[t]{4}{*}{ Vertex } & X-Position [m] & -11.70 & $<\overline{\mathrm{X}}<$ & -10.54 & -12.00 & $<\mathrm{X}<$ & -11 \\
\hline & Y-Position [m] & -0.04 & $<\mathrm{Y}<$ & 0.04 & -0.04 & $<\mathrm{Y}<$ & \\
\hline & Z-Position [m] & -0.03 & $<\mathrm{Z}<$ & $\overline{0.02}$ & -0.035 & $<\mathrm{Z}<$ & \\
\hline & $\chi^{2} / D O F$ & & $\chi^{2} / D O F<$ & 10 & & - & \\
\hline \multirow[t]{6}{*}{ Kinematik } & $Q^{2}\left[(\mathrm{GeV} / \mathrm{c})^{2}\right]$ & 2. & $<Q^{2}$ & & 2. & $<\bar{Q}^{2}$ & \\
\hline & $\nu[\mathrm{GeV}]$ & 100. & $<\nu$ & & 50. & $<\nu$ & \\
\hline & $\overline{Y_{B j}}$ & & $\overline{Y_{B j}<}$ & 0.85 & & $\overline{Y_{B j}<}$ & \\
\hline & $X_{B j}$ & 0.003 & $<X_{B j}$ & & 0.003 & $<X_{B j}$ & \\
\hline & $W^{2}\left[\mathrm{GeV}^{2}\right]$ & 100. & $<W_{2}$ & & 50. & $<W_{2}$ & \\
\hline & $\theta_{\mu}[\mathrm{rad}]$ & 0.003 & $<\theta_{\mu}$ & & 0.003 & $<\theta_{\mu}$ & \\
\hline
\end{tabular}

Tabelle 5.1: Parameter der Ereignisselektion

\begin{tabular}{|l|r|}
\hline Myonen & $\sim 40 \times 10^{9}$ \\
LAT-Trigger & $\sim 12 \times 10^{5}$ \\
Filter & $40 \times 10^{4}$ \\
Vorselektion & $31 \times 10^{3}$ \\
Auswablkriterien I & $18 \times 10^{3}$ \\
Auswahlkriterien II & $11 \times 10^{3}$ \\
\hline
\end{tabular}

Tabelle 5.2: Statistik zur Reduktion des Datensatzes 
rekonstruierten Monte-Carlo-Ereignisse beträgt $19 \mathrm{k}$ ( Auswahlkriterien I) bzw. 15 $k$ (Auswahlkriterien II).

\subsection{Die Auswahl der primären hadronischen Spuren}

Ziel der Spurselektion ist es :

1. Möglichst alle am primären Vertex entstandenen, geladenen Hadronen aus der Zahl aller enstandenen Teilchen herauszufiltern und

2. Spuren, deren Impuls und Trajektorie nicht zuverlässig bestimmt werden können, zu verwerfen.

$\mathrm{Zu}$ diesem $\mathrm{Zweck}$ wurde folgendermaßen vorgegangen: $\mathrm{Zu}$ jeder rekonstruierten Spur eines Monte-Carlo-Ereignisses wird aus allen generierten Spuren diejenige ausgewählt, deren Parameter am besten mit denen der rekonstruierten Spur übereinstimmen. Anschließend wird die relative Abweichung zwischen 'wahrer' und rekonstruierter Größe gebildet $\left(\left(X_{\text {generiert }}-X_{\text {rekonstruiert }}\right) / X_{\text {generiert }}\right)$ und anhand dieser Abweichung ein Kriterium festgelegt, daß auf weniger gut bzw. falsch rekonstruierte Spuren sensitiv ist. Abbildung 5.5 erläutert diesen Sachverhalt am Beispiel der hadronischen Variablen $P_{t}^{2}$. Dargestellt ist $\left(P_{t_{\text {generiert }}}^{2}-P_{t_{\text {rekonossuiert }}}^{2}\right) / P_{t_{\text {generiert }}}^{2}$. Der Bereich $\left|\left(P_{t_{g e n e r i e r t}}^{2}-P_{t_{\text {rektonstruiert }}}^{2}\right)\right| / P_{t_{\text {generiert }}}^{2}>0.3$ ist sicherlich mit schlecht rekonstruierten Spuren angereichert. Wird weiterhin das Verhältnis dieser Klasse zu allen Spuren für selektionsgeeignete Spurparameter dargestellt, z.B. für den Abstand zum primären Vertex (vgl. Abb.:5.6), ergibt sich hieraus die Möglichkeit, geeignete Spurselektionskriterien abzuleiten. In diesem Beispiel erkennt man ein starkes Ansteigen des Verhältnisses bei einem Spurabstand von ca. 2-3 mm vom primären Vertex. Dies bedeutet, daß weniger gut bzw. falsch rekonstruierte Spuren durch einen Schnitt auf den minimalen Abstand zum Vertex unterdrückt werden können. Bei einem Abstand von ca. $10 \mathrm{~mm}$ fallen bereits $50 \%$ aller Spuren in die Klasse der Spuren, deren Transversalimpuls mehr als $30 \%$ vom tatsächlichen abweicht.

In gleicher Weise wurden die übrigen Spurparameter untersucht, wie z.B. $1^{2}$ der Spuranpassung, Anzahl und Typ der im Fit berücksichtigten Kammerebenen, Durchtrittsorte, relativer Fehler der Impulsbestimmung... . Gleichzeitig wird auf diesc Art nach geometrieabhängigen, d.h. Kammerort - abhängigen Auffälligkeiten gesucht.

Die mittels des Simulationsprogrammes herausgearbeiteten Spurauswahlkritcricn sind nur direkt auf die Datenverteilungen anwendbar, falls sich diese genau entsprechen. Abbildung 5.7 zeigt die Verteilungen des minimalen Abstandes der Spuren zum 


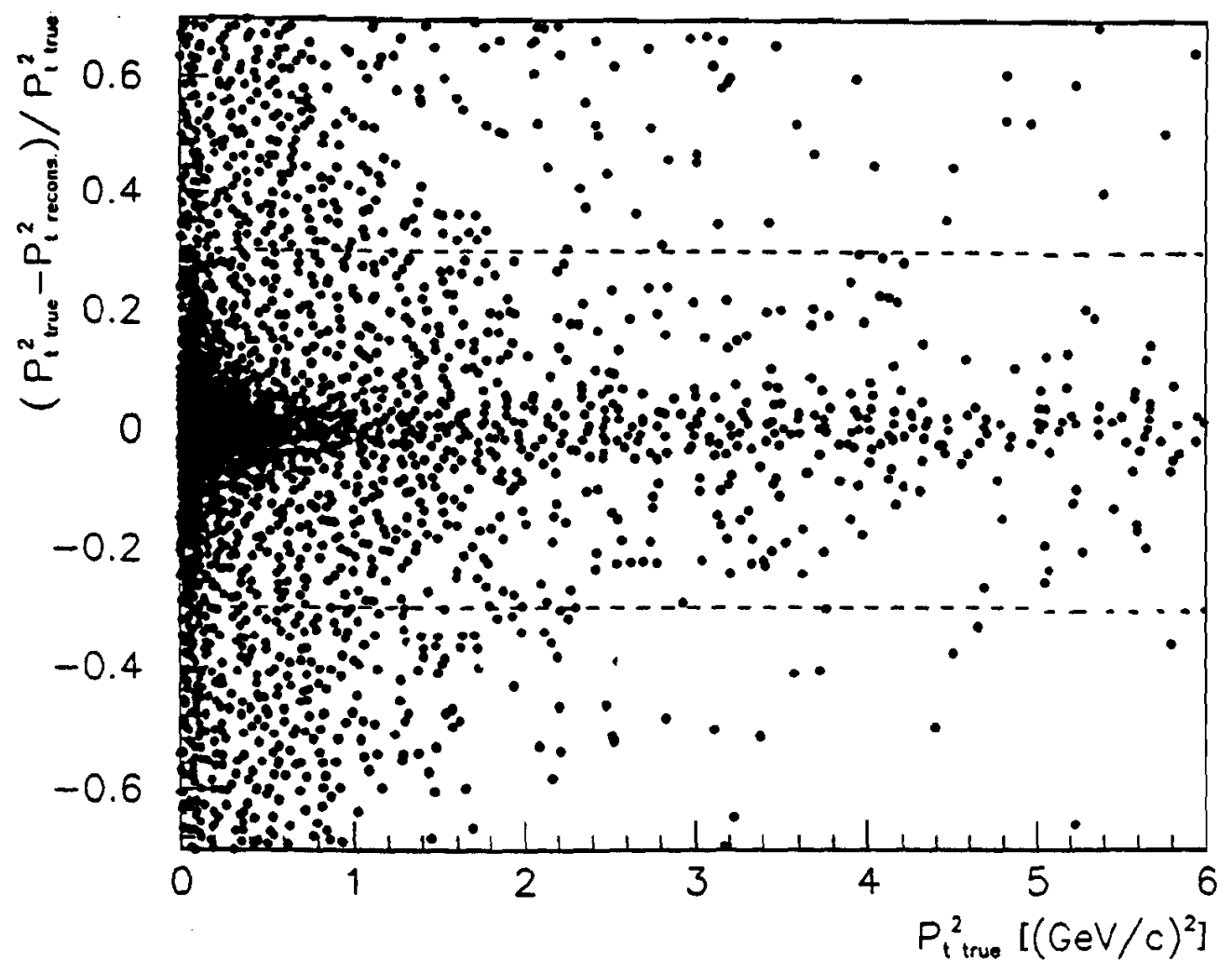

Abbildung 5.5: $\mathrm{P}_{T}^{2}$-Auflösung für hadronische Spuren 


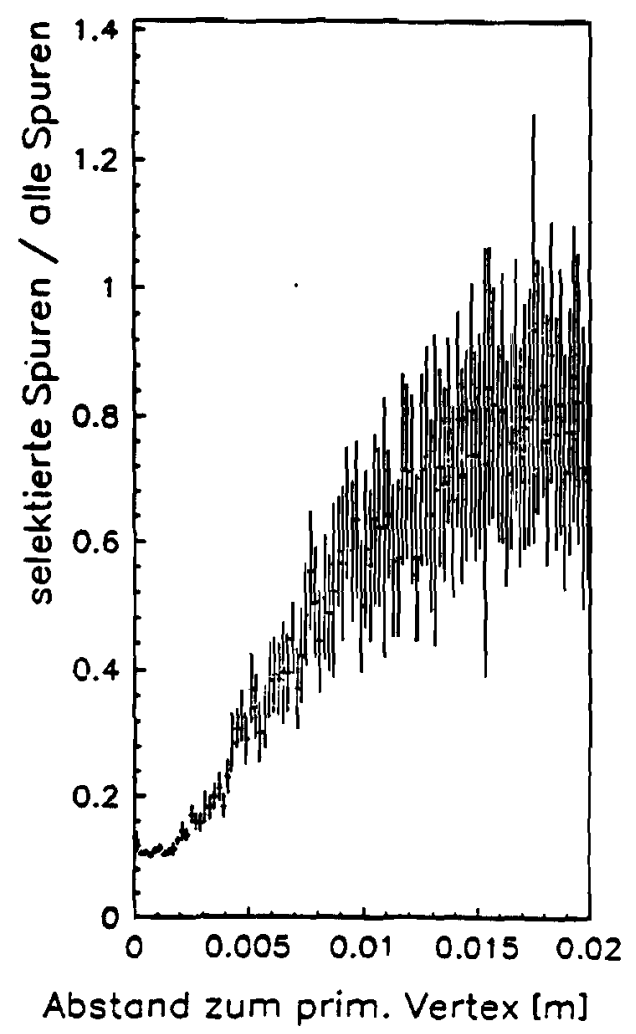

Abbildung 5.6: Verhältnis der 'ungenau' rekonstruierten hadronischen Spuren zu allen hadronischen Spuren in Abhängigkeit vom Raumabstand zum primären Vertex. 
primären Vertex für a) Daten und b) Simulation. Die Übereinstimmung in der Form der Verteilung kann als gut bezeichnet werden, allerdings ist die Verteilung der $\mathrm{Si}$ mulationsrechnung etwas schmaler als die Datenverteilung. Unter Berücksichtigung dieser Abweichung wird das aus der Monte-Carlo-Rechnung gewonnene Schnittkriterium etwas abgeschwächt.
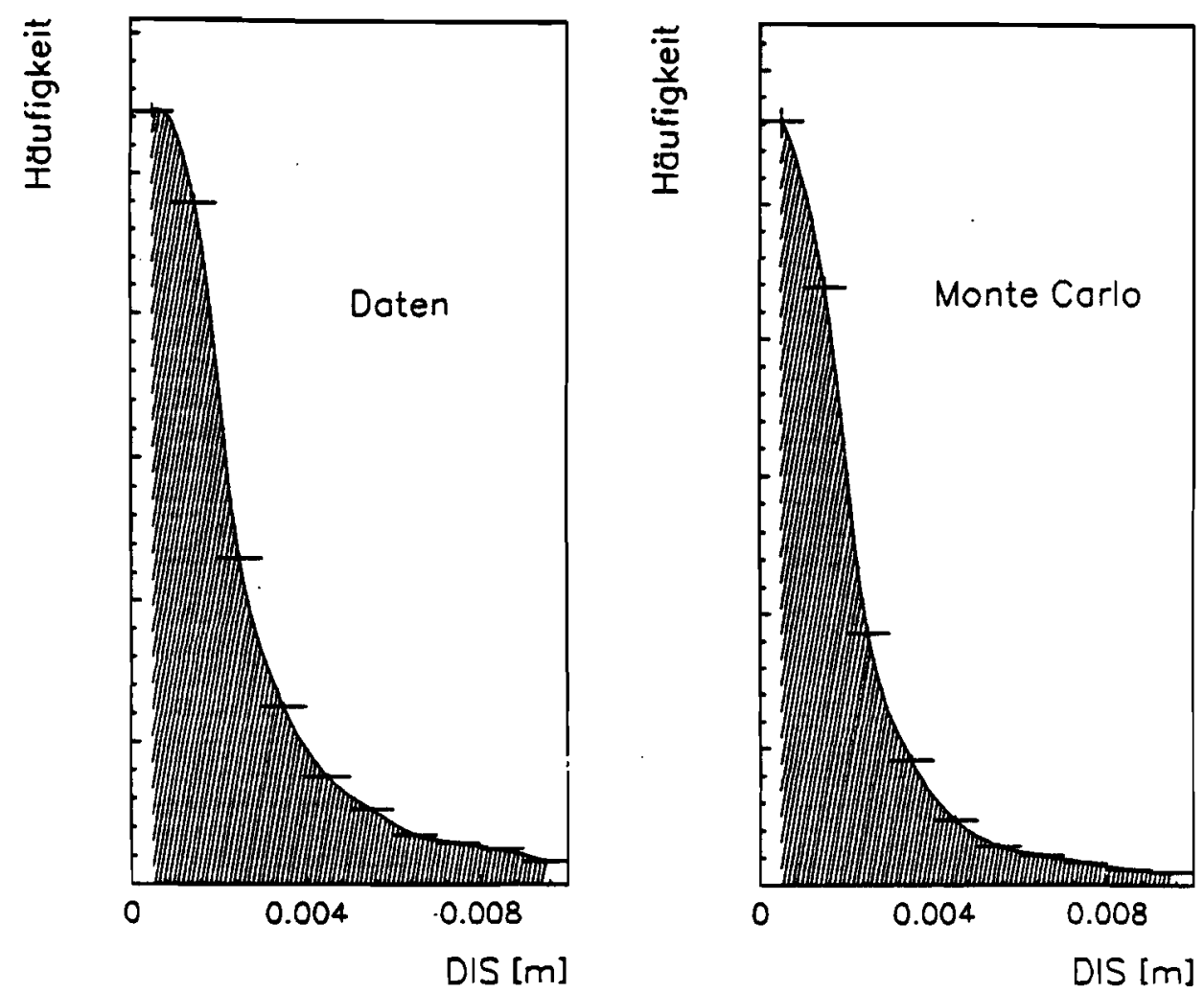

Abbildung 5.7: Abstand der hadronischen Spuren vom primären Vertex für a) Daten und b) Simulation.

Zur Kontrolle der systematischen Unsicherheiten aufgrund dieser Auswahlkriterien werden auch in der Spurselektion zwei Sätze von Schnittparametern gewählt (vgl. Tabelle 5.3).

Die so bestimmten Auswahlkriterien wirken sich nicht nur auf die Qualität der Spuren, sondern auch auf die Identität aus. So werden z.B. Spuren, die aus Zerfallen (z.B. $\Lambda$ und $K_{0}$ ) herrühren oder eine sekundäre Wechselwirkung durchgeführt haben, stark unterdrückt (vgl. Abschnitt 5.4 Korrekturen und systematische Fehler ).

Unter dem Gesichtspunkt der Größe der Akzeptanzkorrektur werden Spuren mit einem Impuls kleiner $10 \mathrm{GeV} / \mathrm{c}$ von der Analyse ausgeschlossen. Dies entspricht bei 
einem minimalen $\nu$ von $100 \mathrm{GeV}$ einem $z_{h}$ von 0.1 . Speziell der Bereich kleiner $z_{h}$ ist mit Zerfallsprodukten und Teilchen aus sekundären Wechselwirkungen stark angereichert. Zur Vermeidung erheblicher Korrekturen in diesem Bereich wurde für alle verwendeten Spuren ein minimaler Energiebruchteil von $z_{h}>0.1$ gefordert. Einen vollständigen Überblick der angebrachten Schnitte zur Spurauswahl gibt Tabelle 5.3. Die mit 'Studie' gekennzeichnete Spalte enthält einen weiteren Satz weniger einschränkender Selektionskriterien, die zur Abschätzung des systematischen Fehlers aufgrund der Spurselektion Verwendung finden.

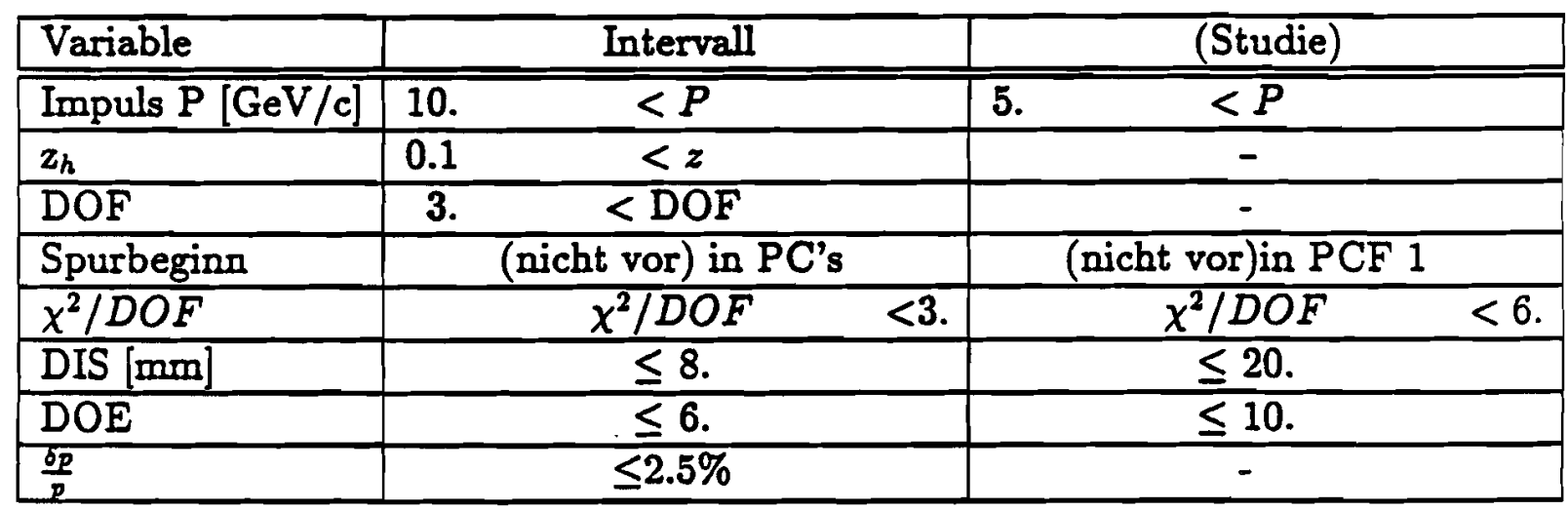

Tabelle 5.3: Parameter der Spurselektion

\subsection{Korrekturen und systematische Fehler}

\subsubsection{Die Akzeptanzkorrektur}

Nicht alle Spuren geladener Hadronen können im Detektor nachgewiesen werden, da:

1. Bereits bei Nichtnachweis des Myons das gesamte Ereignis und damit alle Hadronspuren verloren werden.

2. Nicht alle Teilchen aufgrund der Detektorgeometrie erfaßt werden.

3. Die Nachweiswahrscheinlichkeiten der Detektoren, die die Ortsinformationen liefern, kleiner $100 \%$ sind.

4. Die Rekonstruktionswahrscheinlichkeit der Algorithmen kleiner $100 \%$ ist.

Eine Korrektur auf diese Effekte wird mit Hilfe des Simulationsprogrammes durchgeführt. Alle oben aufgeführten Effekte werden möglichst realistisch im Simulationsprogramm nachgebildet. Die Rekonstruktionsalgorithmen sind identisch mit denen, 
die auf die Daten angewendet werden. Die Akzeptanzkorrekturfunktion $\left(\mathcal{C}_{\mathrm{A}}\right)$ wird aus dem Verhältnis der Anzahl der generierten $\left(\mathcal{N}_{\text {Gen. }}\right)$ zur Anzahl der akzeptierten $\left(\mathcal{N}_{\text {Akz. }}\right)$ Größen gebildet, wobei $x$ die zu korrigierende Variable darstellt. Für die normierten Verteilungen ergibt sich somit:

$$
\mathcal{C}_{A}(x)=\left(\frac{1}{\mathcal{N}_{\mu \mathrm{Gen} .}} \frac{d \mathcal{N}_{\text {Gen. }}^{h}}{d x}\right) /\left(\frac{1}{\mathcal{N}_{\mu A k z .}} \frac{d \mathcal{N}_{\text {Akz. }}^{h}}{d x}\right)
$$

Die im Simulationsprogramm enthaltenen Prozesse der Teilchenzerfalle und sekundären Wechselwirkungen werden über die Akzeptanzkorrektur mitberücksichtigt. In den generierten Verteilungen werden im wesentlichen nur primäre Hadronen berücksichtigt (Pion,Kaon,Proton, sowie Pionen, die im $\rho^{0}$ ihren Ursprung haben), in den Verteilungen der akzeptierten Ereignisse jedoch alle rekonstruierten Hadronen, die die Selektionskriterien in Tabelle 5.3 erfüllen.

Der statistische Fehler dieser Korrektur ist aufgrund der Korrelationen zwischen generierten und akzeptierten Verteilungen schwierig exakt $z \mathbf{u}$ bestimmen. In dieser Analyse wird der statistische Fehler der Akzeptanzkorrektur nach Gaußscher Fehlerfortpflanzung bestimmt und damit die Verteilungen der generierten und akzeptierten Ereignismenge im entsprechenden $x$-Bin als unkorreliert angenommen. Da 'Smearing'8-Effekte und die Korrekturen auf Zerfälle und sekundäre Wechselwirkungen simultan mit der Akzeptanzkorrektur durchgeführt werden, ist die Annahme, $\mathrm{daß}$ die Mengen der generierten und rekonstruierten hadronischen Spuren vollständig korreliert sind, sicherlich nicht gerechtfertigt. Im Vergleich des statistischen Fehlers für unkorrelierte und korrelierte Ereignismengen ergibt sich der Fehler für unkorrelierte Mengen etwas größer.

Als Beispiel für die Größenordnung der Akzeptanz bzgl. einer Ereignisvariablen sei auf die $W^{2}$-Akzeptanzverteilung $\left(\frac{1}{\mathcal{C}_{A}}(x)\right)$ in Abbildung 5.3 hingewiesen. Die Akzep$\operatorname{tanz}$ ist flach und liegt deutlich höher als $80 \%$. Der EinfluB der Akzeptanzkorrektur in den Ereignisvariablen auf die hadronischen Verteilungen ist ohnehin gering. da in der Analyse nur Verteilungen betrachtet werden, die entweder auf die Anzahl der Ereignisse normiert sind oder Mittelwerte hadronischer Größen, z.B. $\left\langle P_{T}^{\prime 2}\right\rangle$ darstellen.

Die über den kinematischen Bereich des Myons integrierten Korrekturfunktionen für a) $\mathrm{z}$ und $\mathrm{b}) \mathrm{P}_{T}^{2}$ sind in Abbildung 5.8 dargestellt. Die Korrektur für $\mathrm{z}$ kanı im Rahmen der statistischen Fluktuationen als flach bezeichnet werden und betrïgt ca. 35\%. Für $\mathrm{P}_{T}^{2}$ ist die Korrektur ähnlich groß, allerdings nehmen die statistischen

\footnotetext{
${ }^{8}$ Aufgrund der endlichen Aufïösung des Spektrometers werden Ereignis- und Spurgrößen nach der Rekonstruktion nicht im gleichen Intervall abgebildet, in dem sie erzeugt wurden, sondern teilweise in benachbarten Bereichen. Speziell bei steil abfallenden Verteilungen kann dies zu nicht unerheblichen Effekten in der Akzeptanzkorrektur führen.
} 

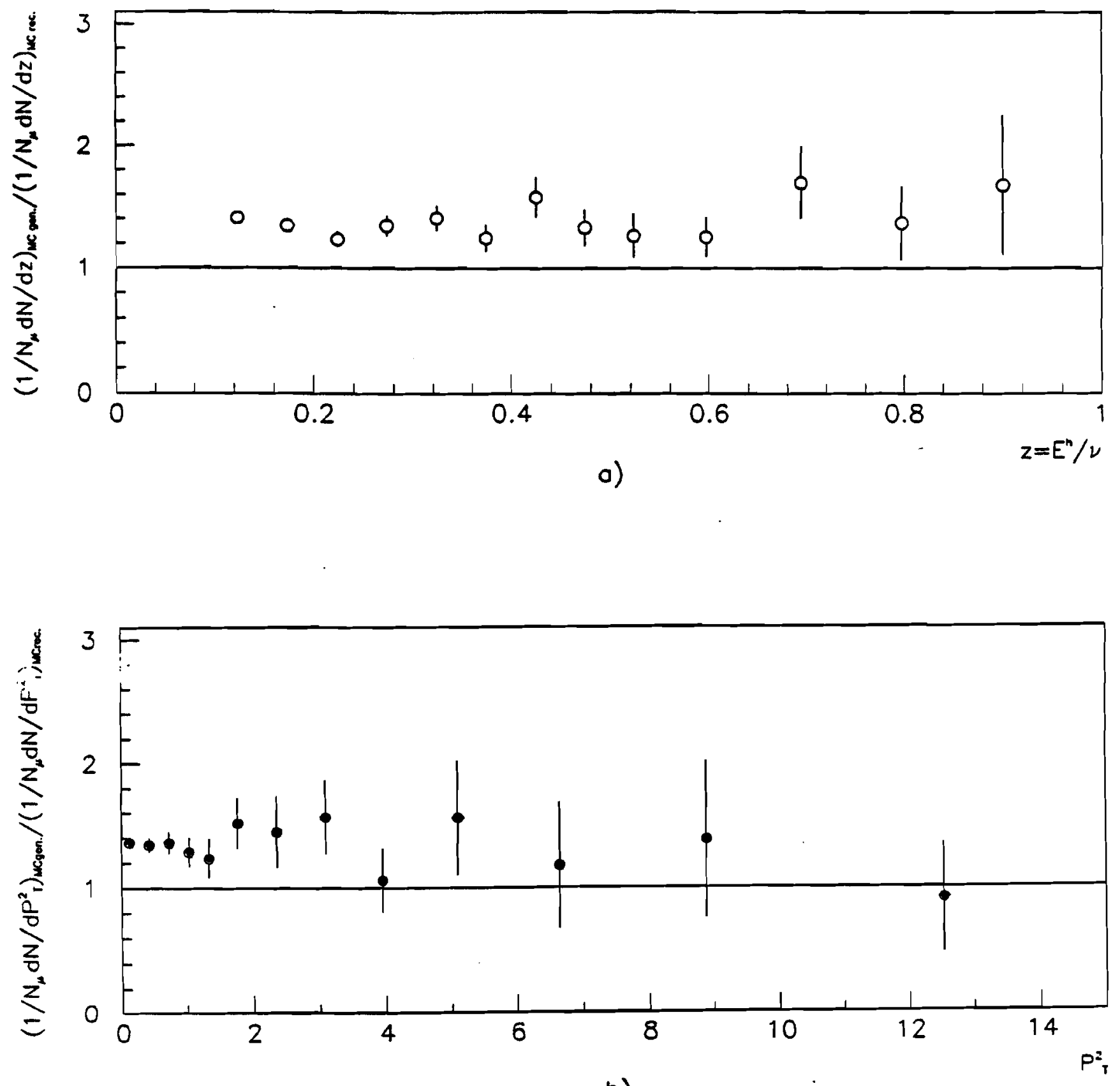

b)

Abbildung 5.8: Akzeptanz-Korrekturfunktionen für a) z und b) $\mathrm{P}_{T}^{2}$ 
Unsicherheiten mit zunehmendem $\mathrm{P}_{T}^{2}$ stark zu.

\subsubsection{Strahlungskorrekturen}

Im Streuexperiment werden nicht nur Ereignisse des Ein-Photon-Austausch gemessen, sondern auch QED-Prozesse höherer Ordnung. Abgestrahlte Photonen vom ein- bzw. auslaufenden Myon (vgl. Abb.:5.9 b) und c)) ändern die Kinematik des Prozesses. Da diese im allgemeinen aus den gemessenen Myonimpulsen abgeleitet wird, ergibt sich z.B. aufgrund der Bremsstrahlung eine Verfälschung. Prozesse des Types f), h) (Vertexkorrektur und Vakuumpolarisation) liefern un-

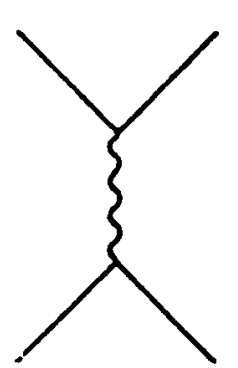

a)

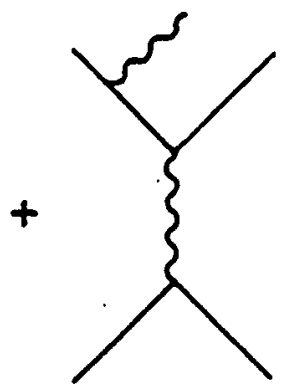

b)

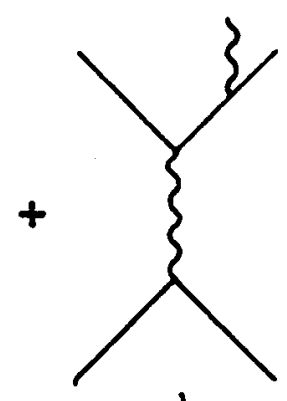

c)

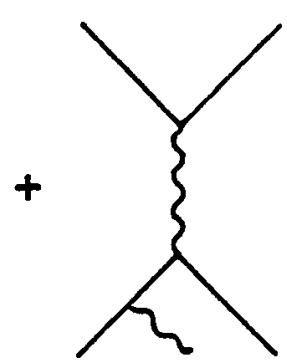

d)

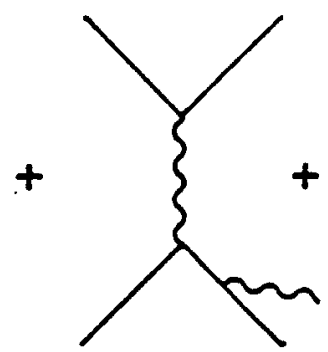

e)

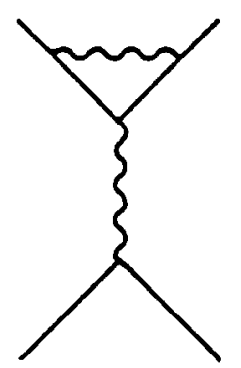

f)

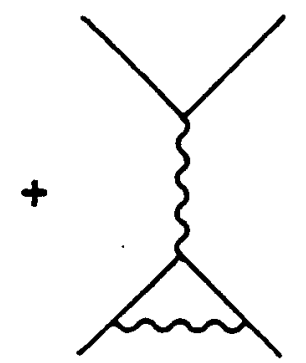

g)

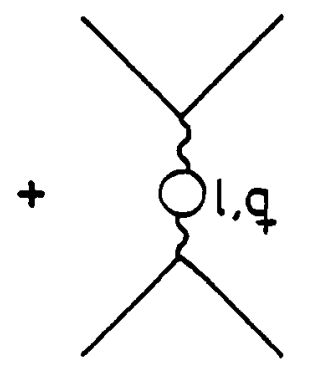

h)

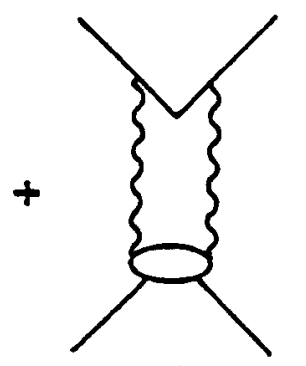

i)

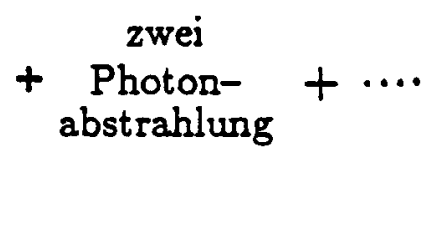

Abbildung 5.9: Ein-Photon-Austausch Feynman-Diagramm + QED-Prozesse höherer Ordnungen

erwünschte Beiträge zum Wirkungsquerschnitt. Klarerweise ergeben sich aus den QED-Prozessen höherer Ordnungen nicht nur Störungen im Wirkungsquerschnitt, sondern auch in der hadronischen Variablen. Der Energiebruchteil z der Hadronen wird durch den Unterschied zwischen scheinbarem und tatsächlichem Energieübertrag des virtuellen Photons gestört, der transversale Impuls $P_{t}$ der Hadronen zusätzlich durch die veränderte Referenz-Richtung des 'wahren' virtuellen Photons. 
Eine Berücksichtigung dieser Störungen wird in der vorliegenden Analyse mit Hilfe des in der EMC entwickelten GAMRAD-Programmes durchgeführt [70]. Dieses Programm basiert auf Rechnungen von Mo \& Tsai [71]. Neben dem Strahlungskorrekturfaktor $R$, der das Verhältnis vom Ein-Photon-Austausch-Wirkungsquerschnitt zum Wirkungsquerschnitt aller in den Korrekturen berücksichtigten QED-Prozessen angibt ( $\left.\mathrm{R}=\frac{\sigma_{1 \gamma}}{\sigma_{\text {gemesesen }}}\right)$, liefert das GAMRAD-Programm das vollständige Spektrum der Bremsstrahlungsphotonen, d.h. die Wahrscheinlichkeit, daß bei einem Streuproze $\beta$ in einem bestimmten kinematischen Bereich $\left(x_{B j}, y_{B j}\right)$ ein Photon der Energie $E_{\gamma}$ unter dem Azimutalwinkel $\varphi$ und dem Polarwinkel $\Theta$ (relativ zur Richtung des scheinbaren virtuellen Photons ${ }^{9}$ ) emittiert wird. Speziell in der Analyse hadronischer Endzustände ermöglicht diese Kenntnis eine Korrektur der gestörten Hadronvariablen. Im GAMRAD-Programm setzt sich der 'gemessene' Wirkungsquerschnitt aus dem Term der Born-Approximation, modifiziert durch einen Korrekturterm $\delta$, der u.a. die Korrekturterme der Vertexkorrektur und Vakuumpolarisation enthält, und dem Term $\sigma_{\text {tail }}$, der die Wahrscheinlichkeit zur Abstrahlung eines reellen Photons repräsentiert, zusammen:

$$
\sigma_{\text {gemessen }}=\sigma_{1 \gamma}(1+\delta)+\sigma_{\text {tail }}
$$

$\sigma_{\text {tail }}$ beinhaltet Beiträge der Photonabstrahlung aus elastischen, quasielastischen und inelastischen Prozessen. In Abbildung 5.10 a) ist der Strahlungskorrekturfaktor sowie b) die kohärenten und c) inelastischen Beiträge zum gesamten im Experiment gemessenen Wirkungsquerschnitt für $x_{B j}=0.5$ und 0.03 gegen $Y_{B j}$ dargestellt.

Strahlungskorrekturen am hadronischen Vertex sind nicht berücksichtigt, da diese allgemein als klein angenommen werden. Beiträge der schwachen Wechselwirkung zum Wirkungsquerschnitt über den Austausch eines $Z_{0}$-Bosons sind ebenfalls nicht enthalten. Neuere Rechnungen zu Strahlungskorrekturen [72], die diese Beiträge berücksichtigen, sind mit den vom GAMRAD-Programm berechneten Korrekturfaktoren konsistent [73], [18]. Kleine Abweichungen ergeben sich im wesentlichen an den kinematischen Rändern, (kleines $\mathrm{x}_{B j}$, hohes $\mathrm{y}_{B j}$ ) in denen der Korrekturterm derart groß ist, daß dieser Bereich ohnehin in dieser Analyse ausgeschlossen wird. Die zugrundeliegenden Annahmen des GAMRAD-Programmes werden nicht nur durch unabhängige Berechnungen bestätigt, sondern auch widerspruchsfrei im Experiment gemessen [74].

Abbildung 5.11 illustriert den Einfluß der radiativen Beiträge auf die hadronischen Variablen $\mathrm{z}$, und $P_{T}^{2}$. Dargestellt ist das Verhältnis aus $1 / N_{\mu} d N^{h} / d x_{\text {'wahr' }}$ und

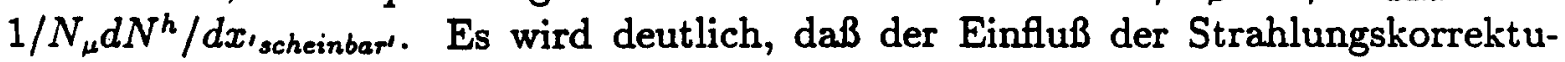

\footnotetext{
9'Scheinbar' bezieht sich auf den Umstand, daB die Kinematik des virtuellen Photons aus den Impulsen des ein- und auslaufenden Myons bestimmt wird. Im Gegensatz hierzu bezieht sich 'wahr' auf die tatsächliche Kinematik des virtuellen Photons während des Austauschprozesses.
} 

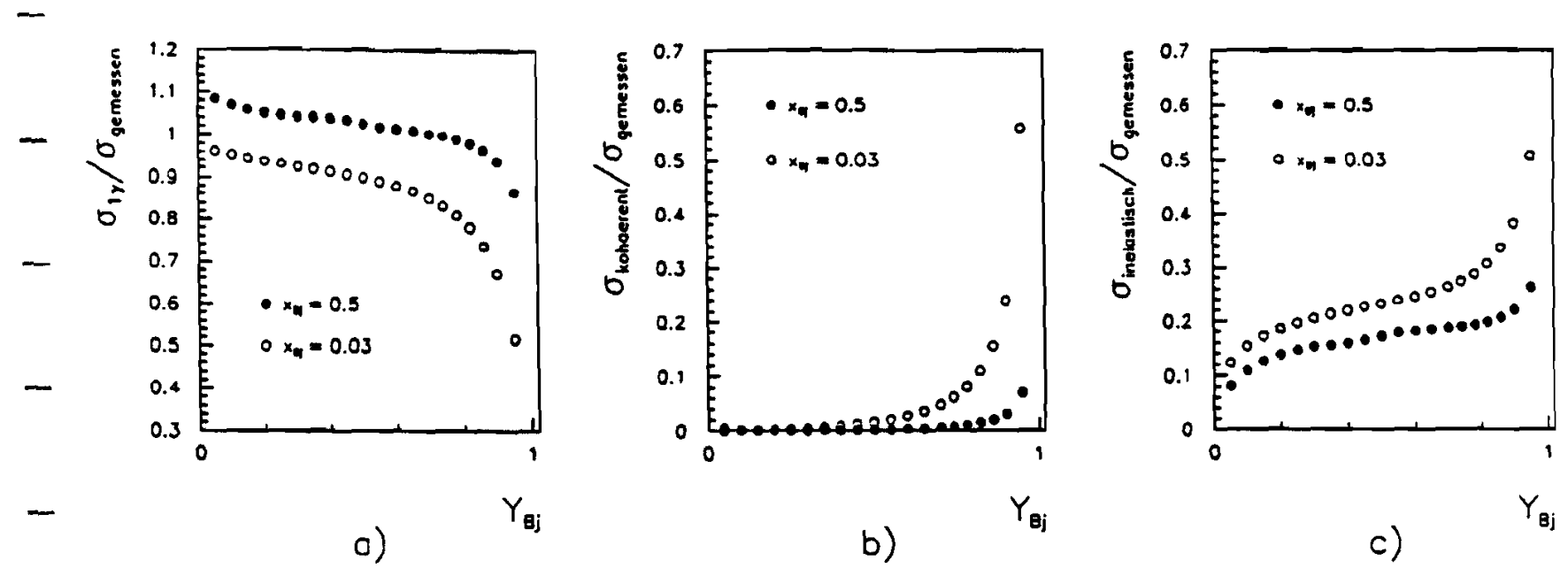

Abbildung 5.10: Strahlungskorrekturfaktor sowie kohärente und inelastische Beiträge zum Wirkungsquerschnitt für $x_{B j}=0.5$ und 0.03 gegen $y_{B j}$.
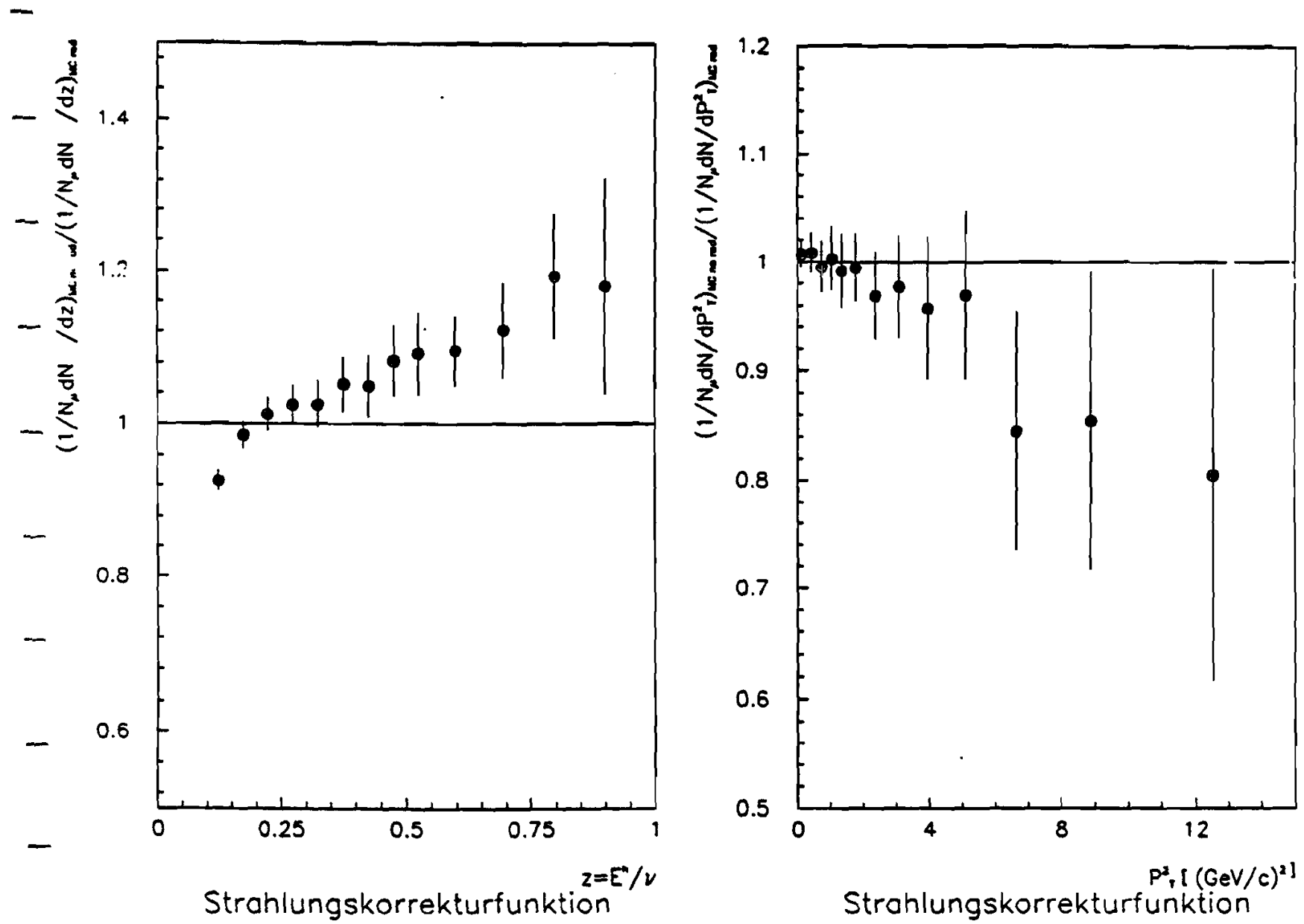

Abbildung 5.11: Strahlungskorrekturfunktion für a) $\mathrm{z}_{h}$ und b) $P_{T}^{2}$ 
ren auf die hadronischen Variablen speziell bei hohen $z$ und $P_{T}^{2}$ nicht unerheblich ist.

Die Strahlungskorrektur wird als weitere Korrektur an die Daten angebracht. Die gesamte Korrektur an die Daten schreibt sich damit zu:

$$
\operatorname{Daten}_{\text {korrigiert }}(x)=\operatorname{Daten}_{\text {roh }^{\prime}}(x) * \mathcal{C}_{A k z .}(x) * \mathcal{C}_{R a d .}(x)
$$

mit:

$$
\mathcal{C}_{A k z .}(x)=\left(\frac{1}{\mathcal{N}_{\mu \mathrm{Gen} .}} \frac{d \mathcal{N}_{\text {Gen. }}^{h}}{d x}\right) /\left(\frac{1}{\mathcal{N}_{\mu A k z .}} \frac{d \mathcal{N}_{A k z .}^{h}}{d x}\right)
$$

und:

$$
\mathcal{C}_{\text {Rad. }}(x)=\left(\frac{1}{\mathcal{N}_{\mu}} \frac{d \mathcal{N}_{\text {twahr' }}^{h}}{d x}\right) /\left(\frac{1}{\mathcal{N}_{\mu}} \frac{d \mathcal{N}_{\text {tscheinbar }}^{h}}{d x}\right)
$$

Als abschließendes Beispiel zum Einfluß dieser Korrektur wird in Abbildung 5.12 die vollständige Korrektur der $\mathrm{Y}_{B_{j}}$-Verteilung gezeigt. Ohne das Anbringen der Kor-

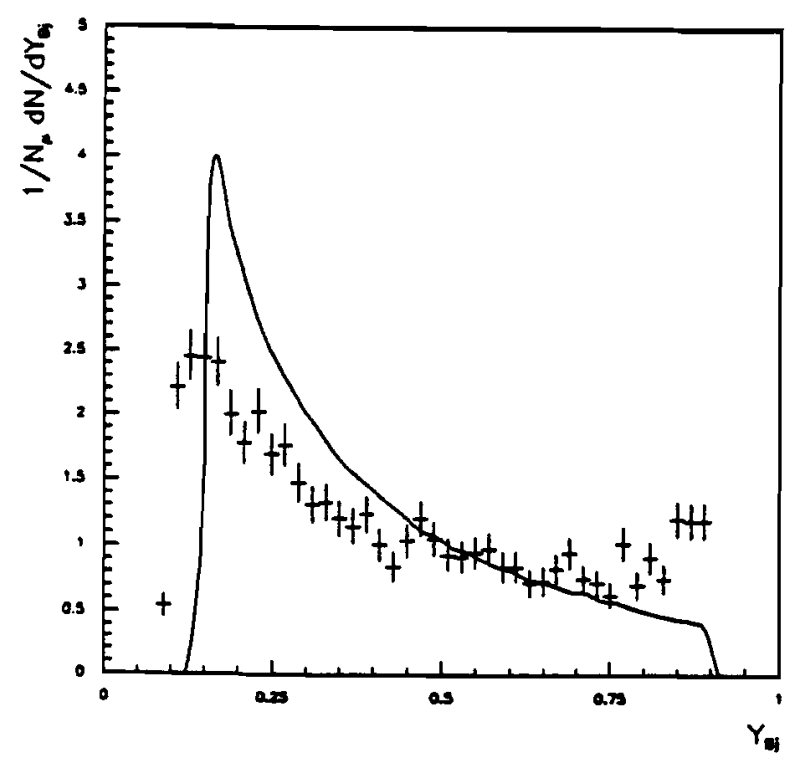

a)

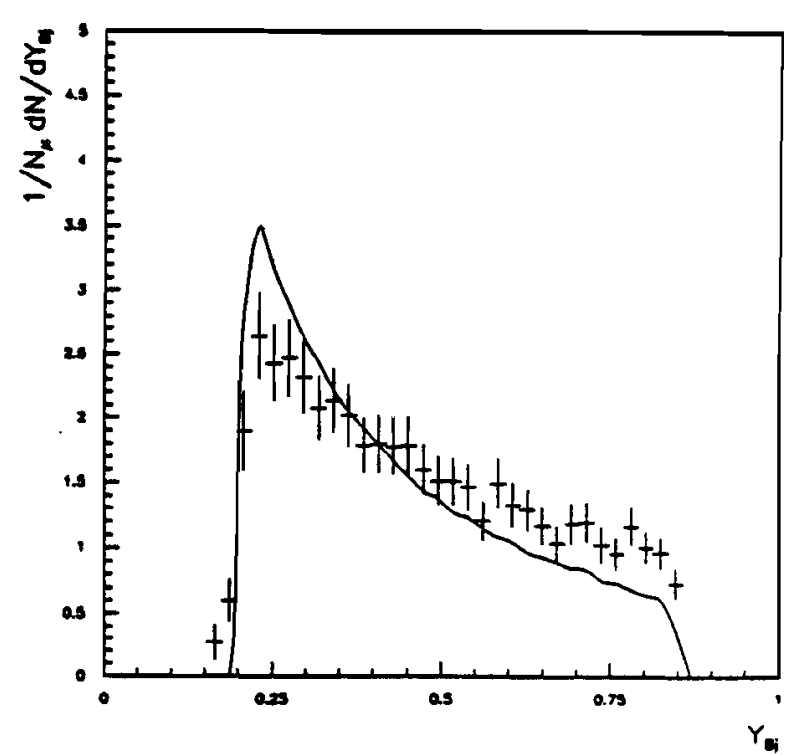

b)

Abbildung 5.12: a) Rohe und b) vollständig korrigierte $Y_{B j}$-Verteilung im Vergleich zum Lund-Modell.

rekturen ist ein deutliches Ansteigen der Verteilung zu großen $Y_{B j}$ hin zu erkennen. Das Anbringen der Strahlungskorrekturen kompensiert diesen Effekt. Bei kleinen $Y_{B j}$ wirkt der Einfluß der Akzeptanzkorrektur. 


\subsubsection{Systematische Fehler}

Die systematischen Fehler der extrahierten, korrigierten Verteilungen setzen sich aus folgenden Beiträgen zusammen:

1. Unsicherheiten und Modellannahmen im Simulationsprogramm.

2. Näherungen und Modellannahmen im Strahlungskorrekturprogramm.

3. Bisher noch nicht näher erläuterte Einflüsse der Elektronen-Kontamination.

Systematische Unsicherheiten in $\mathrm{z}_{h}$

Wie bereits erwähnt, wurde die gesamte Analyse mit zwei Sätzen von Auswahlkriterien durchgefïhrt. Abbildung 5.13 zeigt exemplarisch den Einfluß der unterschiedlichen Auswahlkriterien für die akzeptanz- und strahlungskorrigierte normierte zVerteilung in $W^{2}$-Intervall: $300 \mathrm{GeV}^{2}<W^{2}<400 \mathrm{GeV}^{2}$. Hierbei bezeichnet 'Auswahlkriterien II' den finalen Satz von Auswahlkriterien und 'Auswablkriterien I' jene, die in Tabelle 5.1 und 5.3 mit 'Studie' gekennzeichnet sind. Im Falle einer perfekten Beschreibung aller Details im Simulations- und Strahlungskorrekturprogramm sollten für unterschiedliche Selektionskriterien die korrigierten Datenverteilungen identisch ${ }^{10}$ sein.

Die relative Abweichung der Verteilungen in Abbildung $5.13-\Delta=\frac{d \|\left(z_{\text {tight }}-z_{\text {loose }}\right)}{d z_{\text {tight }}}$ - variiert zwischen $10 \%$ (bei kleinen und großen z) und $\sim 1 \%$ (bei mittleren $z$ ). Der Mittelwert über alle Bins ergibt sich für dieses $W^{2}$-Intervall $z u \cong 6 \%$. Im anschließenden $\mathrm{W}^{2}$-Intervall $400 \mathrm{GeV}^{2}<W^{2}<600 \mathrm{GeV}^{2}$ steigt dieser mittlere Wert auf $\cong 14 \%$.

Die Variation der Unsicherheiten mit 2 sind konsistent mit den Beiträgen der Korrekturen. Bei kleinen $\mathrm{z}$ sind die Einflüsse der Zerfallsprozesse und sekundären Wechselwirkungen ausgeprägt, bei hohen $z$ die Strahlungskorrekturen und damit eine zunehmende Elektronen-Kontamination aus konvertierenden energiereichen Bremsstrahlungsphotonen.

\footnotetext{
${ }^{10} \mathrm{Abgesehen}$ von kleinen statistischen Unterschieden
} 


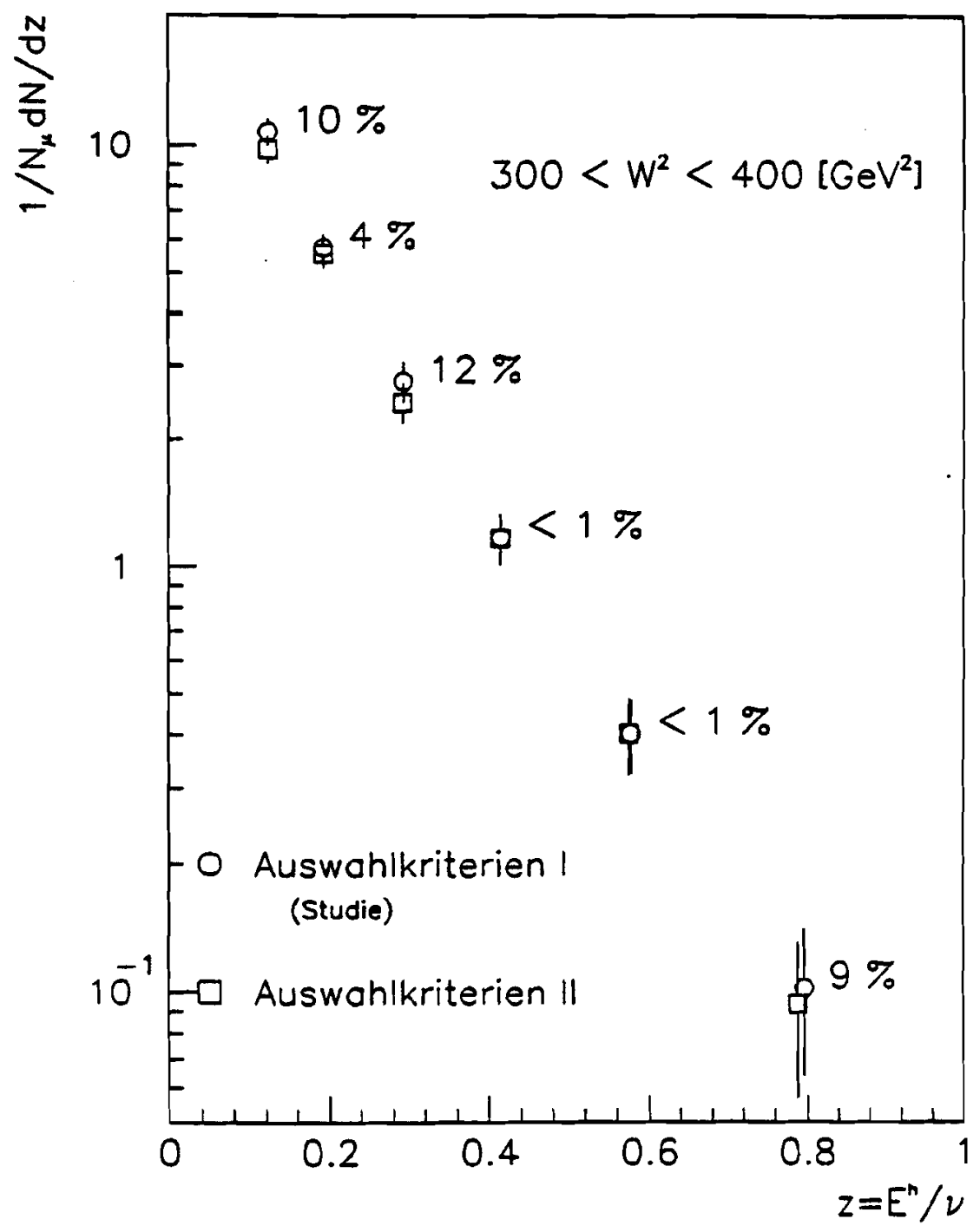

Abbildung 5.13: Akzeptanz- und strahlungskorrigierte normierte z-Verteilung im $W^{2}$-Intervall $300 \mathrm{GeV}^{2}<W^{2}<400 \mathrm{GeV}^{2}$ für zwei unterschiedliche Sätze von Auswahlkriterien. 
Systematische Unsicherheiten in $P_{T}^{2}$ und $\left\langle P_{T}^{2}\right\rangle$

Die systematischen Unsicherheiten in $\mathrm{P}_{T}^{2}$ und $\left\langle P_{T}^{2}>\right.$ werden in gleicher Weise bestimmt wie im Falle der z-Verteilungen und sind in Tabelle 5.4 zusammengefaßt. Der systematische Fehler in $\mathrm{P}_{T}^{2}$ wird für den Bereich $\mathrm{P}_{T}^{2}>3.6(\mathrm{GeV} / \mathrm{c})^{2}$ angegeben, da dieser Bereich unter den in Kapitel 2 erläuterten physikalischen Gesichtspunkten für diese Analyse wesentlich ist. Der abgeschätzte systematische Fehler für die $\mathrm{P}_{T^{-}}^{2}$ Verteilungen aufgrund der Akzeptanz- und Strahlungskorrekturen liegt bei 19\%. Der mittlere systematische Fehler in $\left\langle P_{T}^{2}\right\rangle$ variiert zwischen $3 \%$ und $12 \%$, abhängig vom betrachteten $z$-Intervall.

\section{Elektronen-Kontamination}

Die dominanten Quellen des Elektronenuntergrundes sind der $\pi^{0}$-Zerfall und die harte Bremsstrahlung. Der $\pi^{0}$-Zerfall und die Konversion der beiden Zerfallsphotonen in $\mathrm{e}^{+} \mathrm{e}^{-}$-Paare wird im Simulationsprogramm beschrieben und über die Akzeptanzkorrektur berücksichtigt. Die Elektronen aus diesem Prozeß reichern den Bereich kleiner $z$ an, da die gesamte Energie des $\pi^{0}$ beim Zerfall und anschließender Konversion auf vier Elektronen verteilt wird.

Bremsstrahlung ist in der z.Z. verwendeten Monte-Carlo-Version nicht enthalten. Eine Abschätzung der Elektronen-Kontamination aufgrund dieses Prozesses wird im folgenden gegeben ${ }^{11}$ :

Der relative Anteil radiativer Ereignisse nimmt (wie bereits im Abschnitt 5.4.2 erläutert wurde) mit größer werdendem $Y_{B j}$ und abfallendem $X_{B j}$ zu. Bei einem $\mathrm{Y}_{B j}$ von 0.45 (mittleres $\mathrm{Y}_{B j}$ in dieser Analyse) und $\mathrm{X}_{B j}$ von 0.03 (mittleres $\mathrm{X}_{B j}$ im verwendeten kinematischen Bereich) beträgt dieser $\sim 10 \%\left(R_{Y_{B j}=0.45, x_{B j}=0.03} \cong 0.9\right)$. Unter der Annahme, daß in all diesen Ereignissen ein hartes Photon abgestrahlt wird, die Konversion im Deuteriumtarget stattfindet, und die Elektronspuren in den primären Vertex gefittet werden ${ }^{12}$, wird die Anzahl der Konversionselektronen berechnet. Eine Strahlungslänge für flüssiges Deuterium beträgt $7.57 \mathrm{~m}$. Die Konversionswahrscheinlichkeit eines Photons ist gegeben durch $\frac{7}{9} \frac{X}{X_{0}}$. Die Aufteilung der Photonenenergie auf ein Konversionselektron wird für hohe $\gamma$-Energien $\left(E_{\gamma} \geq\right.$ einige

\footnotetext{
${ }^{11}$ Eine vorläufige Analyse der Elektronen-Kontamination unter Verwendung der Informationen des elektromagnetischen Kalorimeters führte z.Z. dieser Analyse zu keinen quantitativ schlüssigen Ergebnissen, da sich Kalibration und Akzeptanzstudien des EM in einem sehr frühen Analysestadium befanden. Qualitativ war ein mit den Erwartungen konsistentes Signal extrahierbar.

${ }^{12}$ Dies führt sicherlich zu einer Überschätzung der Elektronen-Kontamination.
} 
$\mathrm{GeV}$ )beschrieben durch[67]:

$$
g\left(k, E_{+}\right) \approx v^{2}+(1-v)^{2}+\frac{2}{3} v(1-v) \text { für } E \gg m_{e} Z^{-\frac{1}{3}}, v=E_{+} / k
$$

Hierbei ist $k$ die $\gamma$-Energie und $E_{+}$die Positronenenergie.

Die Wahrscheinlichkeit, ein Elektron (Positron) mit einem Energiebruchteil $>0.6 \mathrm{zu}$ finden, ergibt sich damit zu ca. 40\%. Die Anzahl der Hadronen pro Ereignis bei einem $z$ von $>0.6$ liegt bei ${ }^{13} \sim 0.04$. Mit der halben Targetlänge als Konversionsmedium $(=0.57 \mathrm{~m})$ ergibt sich der relative Elektronuntergrund für die oben angegebenen Parameter zu:

$$
\frac{\int_{0.6}^{1.0} d z \frac{1}{N} \frac{d N}{d z_{e}}}{\int_{0.6}^{1.0} d z \frac{1}{N} \frac{d N}{d x_{h}}} \cong \frac{(1-R) \times \frac{7}{9} \frac{x / 2}{x_{0}} \times 2 \times 0.4}{4.0 \times 10^{-2}} \hat{\sim} 10 \%
$$

Diese Abschätzung stimmt mit Ergebnissen detaillierter Studien aus anderen Experimenten mit ähnlichen Gegebenheiten gut überein [68]. Der Einfluß der ElektronenKontamination auf die Transversalimpulsverteilungen ist klein [69].

Die systematischen Fehler aus angebrachten Korrekturen und Elektronenuntergrund ${ }^{14}$ sind in Tabelle 5.4 zusammengefaßt.

\footnotetext{
${ }^{13}$ Die Anzahl ergibt sich aus dem Integral $\int_{0.6}^{1.0} d z \frac{1}{N_{\mu}} \frac{d N}{d z}$, wobei $e^{a+b z}$ an die gemessenen z-
} Verteilungen angepaßt wird. a ergibt sich $z u \sim 3$ und b zu $\sim-7$ (vgl. Abschnitt 6.1).

${ }^{14}$ quadratisch addiert 


\section{Präsentation der Ergebnisse und Diskussion}

In diesem Kapitel werden die im Fermilab-Experiment 665 gemessenen inklusiven Hadronverteilungen präsentiert und diskutiert. Zunächst wird auf die Verteilungen in der longitudinalen Variablen $z$ eingegangen. Die skalierte Energieverteilung der Hadronen wird im Rahmen des QPM und unter den Gesichtspunkten des 'Scaling' und 'environmental independence' diskutiert und mit Verteilungen anderer Experimente verglichen. Schwerpunktmäßig wird anschließend auf die transversalen Eigenschaften des hadronischen Endzustandes eingegangen. Die normierten $\mathrm{P}_{T}^{2}$-Spektren und Verteilungen des mittleren Transversalimpulses werden präsentiert und mit verschiedenen Modellen und anderen Experimenten verglichen ${ }^{1}$.

\subsection{Skalierte Energieverteilungen der Hadronen}

In Abbildung 6.1 ist die auf die Anzahl der tiefinelastischen Ereignisse normierte differentielle Multiplizität des Energiebruchteils z für positive und negative Hadronen dargestellt. Im QPM erwartet man bei kleinem $x_{B j}\left(x_{B j}<0.3\right)$ keine deutlichen Ladungsunterschiede, da die Streuung dominant am ladungssymmetrischen See des Nukleons stattfindet. Darüberhinaus sind Resteinflüsse der Valenz-Quarks bei Streuung am (isoskalaren) Deuteriumtarget geringer als bei Streuung am Protontarget. Das Ladungsverhältnis in Abhängigkeit von $\mathrm{z}$ ist in Abbildung $6.2 \mathrm{im}$ Vergleich zum Lund-Modell dargestellt. Mit ansteigendem $x_{B j}$ und zunehmendem $z$ sollte ein Überschuß für positive Hadronen beobachtbar sein, da mit zunehmendem $x_{B}$ die Streuung dominant an den Valenz-Quarks stattfindet (vgl. Abb.: 2.4), und hier wiederum die Wahrscheinlichkeit für die Kopplung des virtuellen Photons an ein uoder d-Quark quadratisch mit der Ladung eingeht. Der Überschuß sollte für hohe $z$ ausgeprägter werden. Je größer $z$ desto höher ist die Wahrscheinlichkeit, daß clas beobachtete Hadron das gestoßene primäre Quark enthält. Da mit dem vorliegenclen Datensatz aufgrund des niedrigen mittleren $\mathrm{x}_{B j}\left(\left\langle x_{B j}\right\rangle \cong 0.03\right)$ und der limitierten Statistik eine Analyse dieser Art nicht durchgeführt werden kann, sei z.B. auf [iఠ] verwiesen.

Abbildung 6.3 zeigt die inklusiven z-Verteilungen der in diesem Experiment gemessenen geladenen Hadronen im Vergleich zu Messungen der EMC (NA2, [76]). Der hadronische Endzustand wird in beiden Experimenten durch Myon-Produktion erzeugt - in E665 durch Streuung am Deuteriumtarget, im EMC-Experiment durch

\footnotetext{
${ }^{2}$ Generell wird über die azimutale Variable integriert, da Effekte in dieser Variablen im allgenceinen klein sind [75]. Für $\mathrm{z}$ und $\mathrm{P}_{T}^{2}$ wird jeweils über die andere Variable integriert, anderenfalls darauf hingewiesen.
} 


\begin{tabular}{|c|c|c|c|c|}
\hline Variable & Intervall & & $\begin{array}{c}\text { mittlerer } \\
\text { systematischer } \\
\text { Fehler }\end{array}$ & $\begin{array}{c}\text { unter Berücksichtigung } \\
\text { einer } 10 \% \text {-igen Elektronen- } \\
\text { Kontamination }\end{array}$ \\
\hline$z_{h}$ & $0.1<z_{h}<1.0$ & & $15 \%$ & $18 \%$ \\
\hline $\mathrm{P}_{T}^{2}$ & $3.6<P_{T}^{2}<16.0$ & $(\mathrm{GeV} / \mathrm{c})^{2}$ & $19 \%$ & $22 \%$ \\
\hline$\left.<P_{T}^{2}\right\rangle$ & $0.1<z_{h}<0.2$ & & $7 \%$ & $12 \%$ \\
\hline$\left\langle P_{T}^{2}\right\rangle$ & $0.2<z_{h}<0.4$ & & $3 \%$ & $10 \%$ \\
\hline$\left\langle P_{T}^{2}\right\rangle$ & $0.4<z_{h}<1.0$ & & $9 \%$ & $14 \%$ \\
\hline$\left\langle P_{T}^{2}\right\rangle$ & $0.01<z_{h}^{2}<1.0$ & & $12 \%$ & $16 \%$ \\
\hline
\end{tabular}

Tabelle 5.4: Mittlere systematische Fehler in $z_{h}, \mathrm{P}_{T}^{2}$ und $\left\langle P_{T}^{2}\right\rangle$

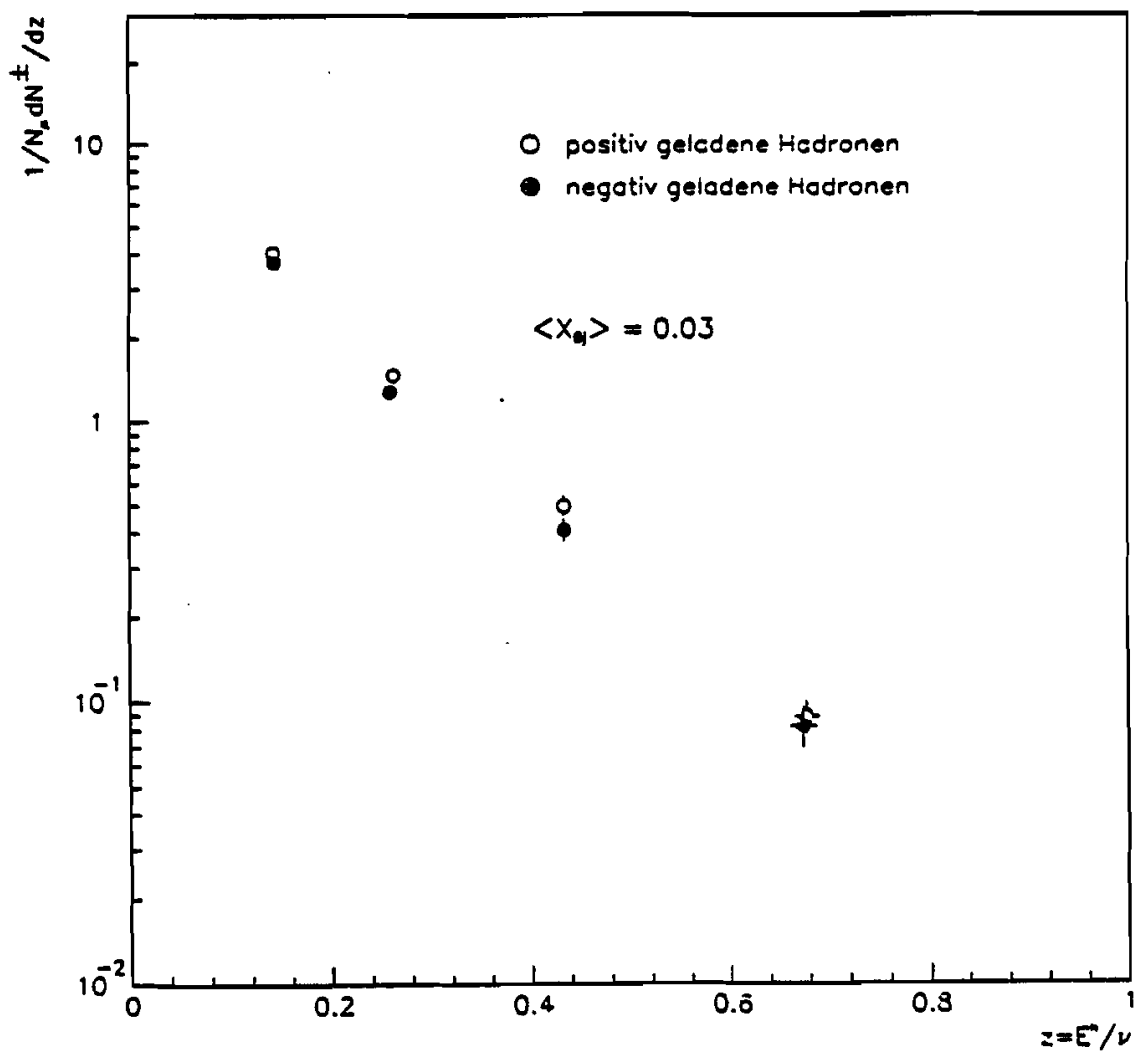

Abbildung 6.1: z-Spektrum für positive und negative Hadronen 


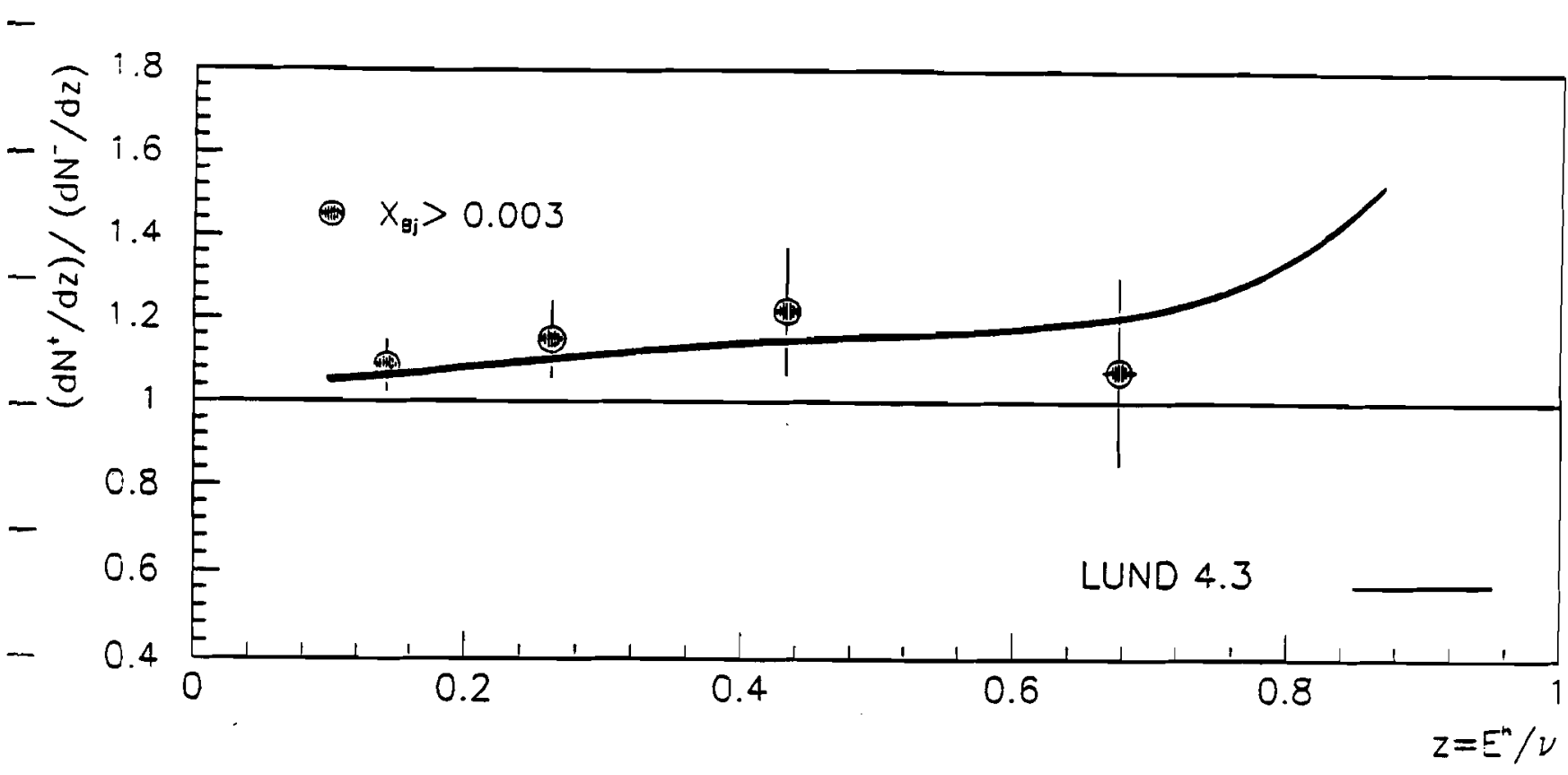

Abbildung 6.2: Ladungsverhältnis in Abhängigkeit von $z$ im Vergleich zum LUND-Modell (Version 4.3)

Streuung am Wasserstoff. Darüberhinaus unterscheiden sich die erfaßten kinematischen Bereiche deutlich. Während im EMC-Experiment bei einer Strahlenergie von $280 \mathrm{GeV}$ ein maximales $\mathrm{W}^{2}$ von ca. $450 \mathrm{GeV}^{2}$ erreicht wurde, liegt in diesem Experiment bereits das mittlere $\mathrm{W}^{2}$ bei $420 \mathrm{GeV}^{2}$.

Die gute Übereinstimmung kann im Rahmen der in Kapitel 2. erläuterten Grundannahmen der Fragmentierungshypothese environmental independence und Feynman scaling verstanden werden: Darüberhinaus kann abgeleitet werden, daß die Skalenbrechung im hadronischen Endzustand nur einen kleinen Effekt darstellt ${ }^{2}$.

Einen deutlichen Unterschied aufgrund der unterschiedlichen Targetmaterialen (EMC: Wasserstoff, E665: Deuterium) erwartet man in den Spektren der Summe der positiven und negativen Hadronen nicht, da die Unterschiede in den Quarkverteilungsfunktionen und damit in den Strukturfunktionen zwischen Deuterium und Wasserstoff klein sind (insbesondere bei kleinen $x_{B j}$ ).

Auch im Vergleich zu den Daten der CHIO-Kollaboration ${ }^{3}$ (vgl. Abb.:6.3) sind keine signifikanten Unterschiede zu erkennen. Dieses Fermilab-Experiment wurde bei einer Strahlmyonenergie von $147 \mathrm{GeV}$ durchgeführt. Die Daten stammen von

\footnotetext{
${ }^{2}$ Relativ zum unterschiedlichen kinematischen Bereich zwischen dem Experiment der EMC und diesem Experiment und im Hinblick auf die betrachtete Variable 2.

${ }^{3}$ Chicago Harvard Inlinois Oxford
} 


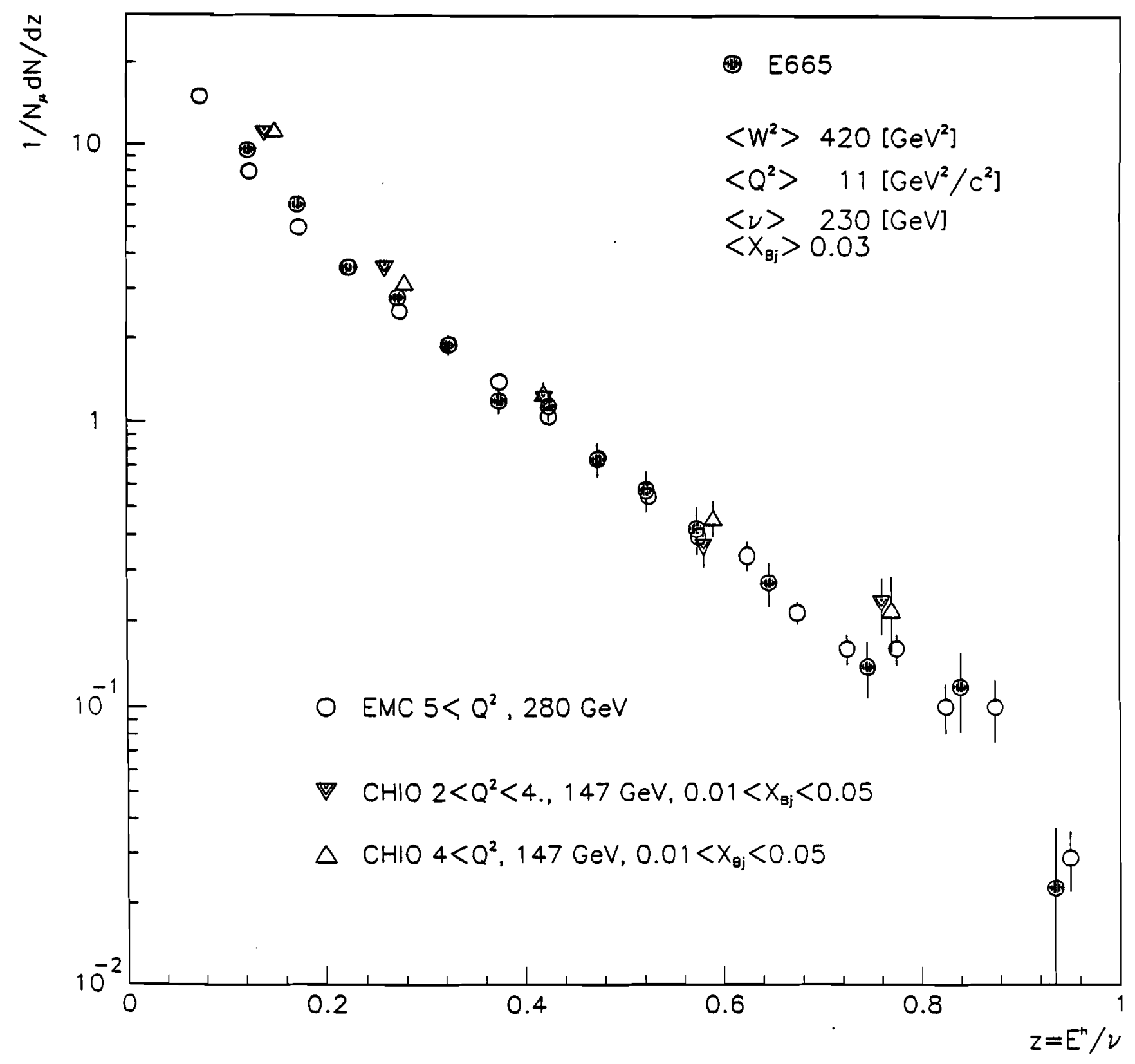

Abbildung 6.3: z-Verteilung für geladene Hadronen gemessen in diesem Experiment im Vergleich zu Messungen der EMC und CHIO 
der Streuung am Deuteriumtarget [77].

In Abbildung 6.4 sind die Daten dieser Analyse im direkten Vergleich zu z-Spektren aus der Neutrino-Nukleon-Streuung gezeigt. Der gemessene Prozeß $\nu N \rightarrow \mu^{-}+h+$ $X\left(\bar{\nu} N \rightarrow \mu^{+}+h+X\right)$ impliziert einen geladenen Austauschstrom (CC-Reaktion $\left.{ }^{4}\right)$, der über das $\mathrm{W}^{+}-\left(\mathrm{W}^{-}-\right)$Boson vermittelt wird. Die Daten ( $\odot$, Abb.:6.4) wurden in einem Experiment am Fermilab an der mit Wasserstoff gefüllten 15-footBlasenkammer genommen [78]. Der überwiegende Teil der Messungen wurde am Breitband-Antineutrino-Strahl genommen, der durch Beschuß eines Aluminiumtargets durch 400-GeV-Protonen initiiert wird [79]. Der Median der Verteilung der invarianten Masse $\mathrm{W}$ wird mit $3.7 \mathrm{GeV}$ angegeben und liegt damit deutlich niedriger als im Experiment 665.

Im Gegensatz zu $\mu$-(e-) Produktion, in dem der Gewichtsfaktor $\epsilon_{i}$ in der Beschreibung der effektiven gewichteten Fragmentationsfunktion z, neben den Ladungsquadraten der beteiligten Quarks, durch deren Impulsdichteverteilungen bestimmt wird, wird in den CC-Reaktionen die Auswahl der am Streuprozeß beteiligten Quarks zusätzlich durch das Ladungsvorzeichen des Austausch-Bosons eingeschränkt. Die dominanten Übergänge in der $\bar{\nu} \mathrm{N}-$ Streuung sind: $W^{-}+u \rightarrow d$ und $W^{-}+\bar{d} \rightarrow \bar{u}$ (vgl. Feynman-Graph in Abb.:6.5).

Die entsprechenden Gewichtsfaktoren $\epsilon_{i}$ sind in Tabelle 6.1 für die Prozesse der Elektroproduktion, CC-Reaktion und $\mathrm{e}^{+} \mathrm{e}^{-}-$Vernichtung nach [80] aufgelistet.

Strangeness- und Charm-ändernde Übergänge ( $u \rightarrow s$ und $\bar{s} \rightarrow \bar{c}$ ) tragen mit $<10 \%$ bei. Die z-Verteilungen beschreiben damit im wesentlichen die Fragmentierung der u-Quarks in Hadronen.

Die überwiegende Anzahl der erzeugten Hadronen sind Pionen. Unter Berücksichtigung von Isospininvarianz und Ladungskonjugation können die Quarkfragmentierungsfunktionen in Relation gebracht werden und erlauben einen direkten Vergleich der Fragmentierungsfunktionen aus den verschiedenen Leptoproduktionen [81], [10].

$$
\begin{aligned}
D_{u}^{\pi^{+}}+D_{u}^{\pi^{-}} & =\left[\frac{d N^{+}}{d z}+\frac{d N^{-}}{d z}\right]_{\nu N}=\left[\frac{d N^{+}}{d z}+\frac{d N^{\pi^{-}}}{d z}\right]_{\nu N} \\
& =\left[\frac{d N^{+}}{d z}+\frac{d N^{-}}{d z}\right]_{\mu N} \approx\left[\frac{d N^{+}}{d z}+\frac{d N^{\pi^{-}}}{d z}\right]_{e+e^{-}}
\end{aligned}
$$

Unter diesem Gesichtspunkt ist die recht gute Übereinstimmung der Daten aus der $\bar{\nu} p$-Streuung und der E665-Daten (über immerhin zwei Größenordnungen) verständlich.

\footnotetext{
${ }^{4}$ Charged Current
} 


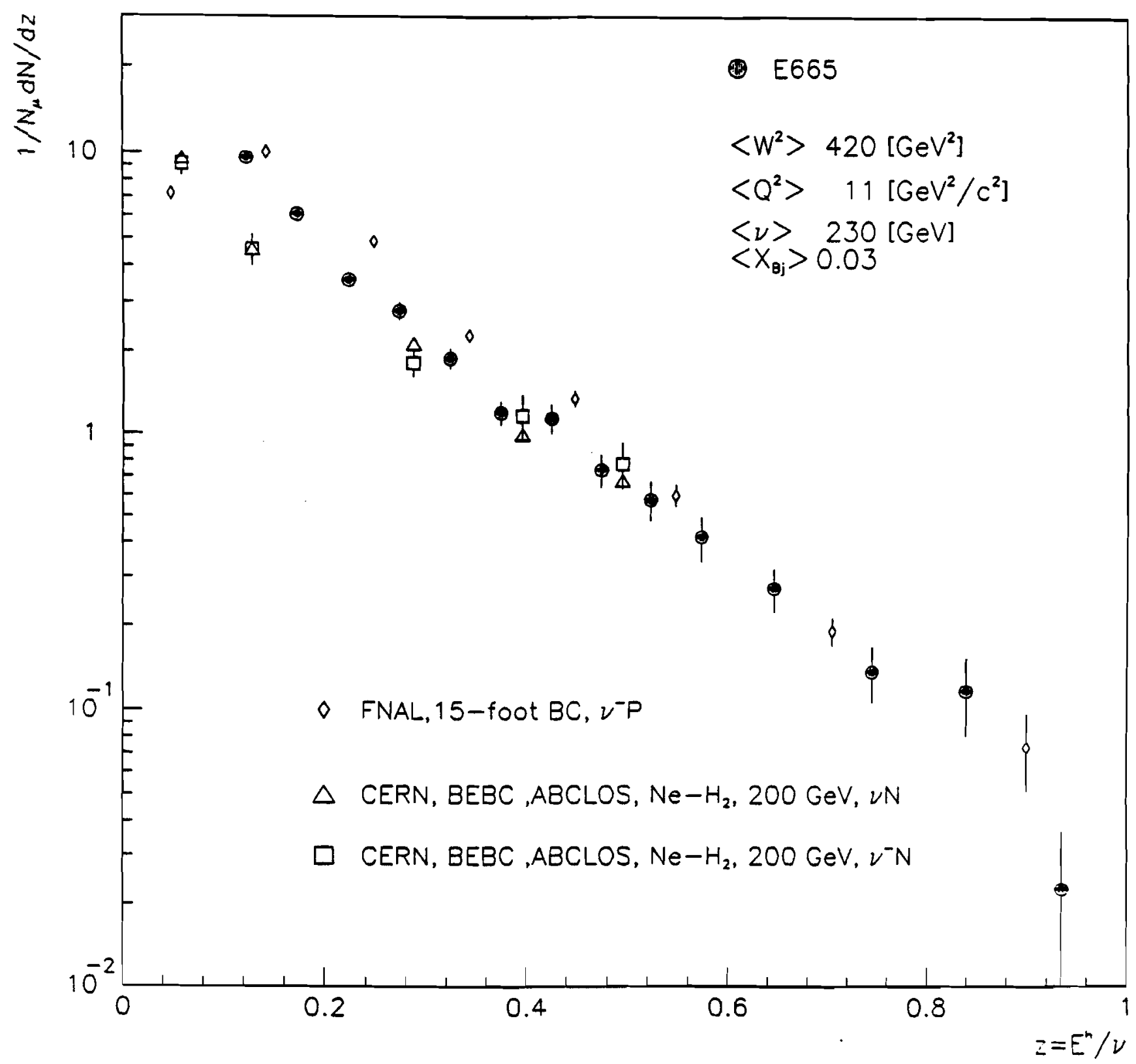

Abbildung 6.4: z-Verteilung für geladene Hadronen gemessen in diesem Expcriment im Vergleich zur $\nu \mathrm{N}-$ und $\bar{\nu} \mathrm{N}-\mathrm{Streuung}$. 


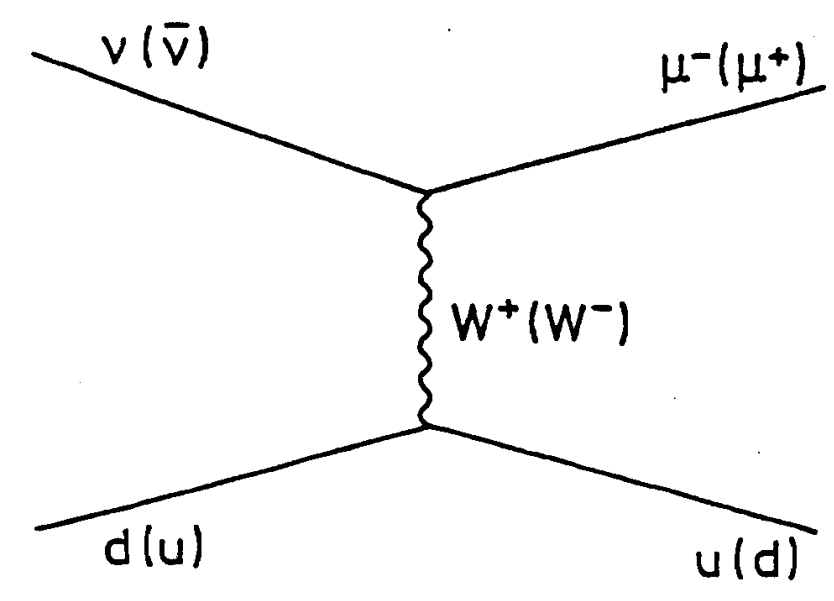

Abbildung 6.5: Feynman-Graph zur CC-Reaktion

\begin{tabular}{|c|c|c|}
\hline $\begin{array}{c}\text { Prozeß } \\
\text { Elektroproduktion }\end{array}$ & & $\begin{array}{l}\text { Yuark-Wahrscheinlichkeiten } \epsilon_{i} \\
\epsilon_{i}=\frac{Q_{i}^{2} f_{i}(x)}{\sum^{2} Q_{i}(x)}\end{array}$ \\
\hline$e^{+} e^{-}-$Vernichtung & & $\epsilon_{i}=\frac{Q_{i}^{2}}{\sum_{i} Q_{i}^{2}}$ \\
\hline Neutrinoproduktion & $\nu:$ & $\epsilon_{u}=\frac{f_{d}(x)}{f_{d}(x)+f_{0}(x)(1-y)^{2}}$ \\
\hline (CC-Reaktion) & & $\epsilon_{d}=\frac{f_{a}(x)(1-y)^{2}}{f_{d}(x)+f_{a}(x)(1-y)^{2}}$ \\
\hline & $\bar{\nu}:$ & $\epsilon_{\bar{u}}=\frac{f_{d}(x)}{f_{d}(x)+f_{u}(x)(1-y)^{2}}$ \\
\hline & & $\epsilon_{d}=\frac{f_{u}(x)(1-y)^{2}}{f_{d}(x)+f_{u}(x)(1-y)^{2}}$ \\
\hline
\end{tabular}

Tabelle 6.1: Quark-Strahl-Zusammensetzung in der tiefinelastischen Leptoproduktion und $e^{+} e^{-}-$Annihilation 
Eine äquivalente Argumentation gilt für den Vergleich der E665-Spektren mit den Daten der $\mathrm{ABCLOS}{ }^{5}$-Kollaboration, die am CERN mit der $\mathrm{BEBC}^{6}$ genommen wurden[82]. Die Kammer war mit einer $74 \%$-igen $\mathrm{Ne} / \mathrm{H}_{2}-\mathrm{Mischung}$ gefüllt, und die Impulsbreite des sekundären $\pi \mathrm{K}$-Strahles betrug $200 \pm 10 \mathrm{GeV}$. Der kinematische Bereich wurde in diesem Experiment eingeschränkt auf: $\mathrm{Q}^{2}>2(\mathrm{GeV} / \mathrm{c})^{2}$ und $\mathrm{W}>4 \mathrm{GeV}$. Darüberhinaus wurde der obere $\mathrm{z}$-Bereich auf $\mathrm{z}<0.6$ beschränkt. Im Bereich $\mathrm{z}>0.2$ (Abb.:6.4) kann eine Übereinstimmung dieser Daten sowohl der $\nu \mathrm{N}$ als auch der $\bar{\nu} \mathrm{N}$ mit den Daten der Myoproduktion festgestellt werden. Allerdings fallen im Bereich $\mathrm{z}<0.2$ nicht unerhebliche Unterschiede (insbesondere zwischen den $\nu$-Experimenten) auf. Dieser Bereich ist jedoch stark durch Targeteigenschaften beeinflußt [82].

Im Rahmen der environmental independence bietet sich ein Vergleich mit Daten der $\mathrm{e}^{+} \mathrm{e}^{-}-$Annibilation an. In Abbildung 6.6 ist die z-Verteilung für die Daten dieser Analyse mit den Verteilungen der $\mathrm{e}^{+} \mathrm{e}^{-}$-Annihilation dargestellt. Für die Daten der $\mathrm{e}^{+} \mathrm{e}^{-}-$Vernichtung ist auf der Ordinate die Größe $\frac{1}{2 \sigma_{\text {tox }}} \frac{d \sigma}{d x_{p}}$ mit $\mathrm{x}_{p}=2 P / W\left(=P / P_{\text {beam }}\right)$ dargestellt. Der Faktor $\frac{1}{2}$ in $\frac{1}{2 \sigma_{\text {too }}} \frac{d \sigma}{d \tau_{p}}$ berücksichtigt, daß die Verteilungen (nur) einer Hemisphäre mit den Daten der $\mu \mathrm{N}$-Streuung vergleichbar sind.

Die Verteilungen der Tasso-Kollaboration [83] wurden bei den Schwerpunktenergien von $\mathrm{W}=14-34 \mathrm{GeV}$ genommen und liegen damit in einem $\mathrm{zu}$ diesem Experiment vergleichbaren Bereich $\left(\left\langle W^{2}\right\rangle_{E 665} \sim 20 \mathrm{GeV}\right.$ ). Die Verteilungen des DELPHI Experimentes am LEP ${ }^{8}$ wurden aus hadronischen Zerfällen des $Z^{0}$-Bosons bei $W=91$ 91.5 GeV [84] genommen.

Der erste Eindruck vermittelt eine recht gute Übereinstimmung der Daten, insbesondere unter Berücksichtigung der völlig verschiedenen Produktionsprozesse und der großen Unterschiede in den Schwerpunktenergien $\left(W_{D E L P H I} / W_{E 665} \sim 4.5\right)$.

Unter der oben angesprochenen Fragmentierungshypothese werden aufgrund der unterschiedlichen Quark-Wahrscheinlichkeiten $\left(\epsilon_{i}\right)$ kleine Unterschiede erwartet. In der $\mathrm{e}^{+} \mathrm{e}^{-}-$Vernichtung sind diese $\epsilon_{i}$ nur von den Quarkladungen abhängig (sofern die Energieschwellen zur Produktion der Quarksorten überschritten sind). In der $\mathrm{e}^{+} \mathrm{e}^{-}$-Annihilation tragen damit deutlich mehr schwere Quarks zur Fragmentierung bei als in der $\mu \mathrm{N}$-Streuung. Die Zerfallsprodukte reichern die $z$-Verteilung zu kleinen z-Werten hin an - die Fragmentierungsfunktion erscheint weicher (d.h. steiler abfallend, energieärmer). Dieser Umstand erklärt einen Teil der in allen Energiebereichen (in $\mathrm{Abb} .66 .6$ ) gegenüber der $\mu \mathrm{N}-$ Streuung steileren Verteilungen $\operatorname{der} \mathrm{e}^{+} \mathrm{e}^{-}-$

\footnotetext{
${ }^{5}$ Aachen Bonn CERN London Oxford Saclay

${ }^{6}$ Big European Bubble Chamber

${ }^{7}$ Detector with Lepton, Photon and Hadron Identification

${ }^{8}$ Large Electron Positron collider
} 
Annihilation. In Abbildung 6.7 ist der Vergleich der z-Verteilungen für beide Produktionsprozesse bei annähernd gleichen invarianten Massen dargestellt. Der gleiche Effekt der steiler abfallenden Verteilungen in der $\mathrm{e}^{+} \mathrm{e}^{-}-$Vernichtung wurde bereits bei niedrigeren Energien (W=14 GeV) beobachtet und detailliert untersucht [85]. Eine weitere Auffalligkeit in Abbildung 6.6 ist, daß die Verteilungen $\left(\mathrm{e}^{+} \mathrm{e}^{-}\right)$mit zunehmender Schwerpunktenergie steiler werden. Dies kann im Rahmen des erweiterten QPM's folgendermaßen interpretiert werden.

Aufgrund der Gluon-Bremsstrahlung (vgl. Abb.:2.6 a)) erhöht sich die Multiplizität auf partonischer Ebene. Das Gluon zerfällt in ein $q \bar{q}-\mathrm{Paar}$, welches seinerseits (neben dem primären gestoßenen Quark) in Hadronen fragmentiert. Die Wahrscheinlichkeit, Hadronen bei hohen $\mathrm{z}$ zu finden, nimmt demzufolge ab und die beobachteten Fragmentierungsfunktionen erscheinen steiler.

Für hohe Transversalimpulse des emittierten Gluons fragmentieren Quark und Gluon als zwei unterscheidbare (Vorwärts-) Jets in Hadronen.

Die Wahrscheinlichkeiten von Quark- und Gluonfragmentierung sind mit Hilfe der Integro-Differentialgleichungen nach Altarelli und Parisi [16],[86],[87] beschreibbar. Wie bereits in Kapitel 2. erläutert, führen diese QCD-Korrekturen zur $\mathrm{Q}^{2}-\mathrm{Abhängigkeit}$ der Fragmentierungsfunktionen (Skalenbrechung) und erfordern einen zusätzlichen $\mathrm{x}_{B} ;$-abhängigen additiven Korrekturterm in der Beschreibung des Produktionsquerschnittes (Faktorisierungsbrechung).

$$
\frac{1}{\sigma_{\text {tot }}} \frac{d \sigma^{h}}{d z}=\sum_{i=u, d, \ldots} \epsilon_{i}\left(x_{B j}, Q^{2}\right) D_{i}^{h}\left(z, Q^{2}\right)+F_{\text {corr }}\left(x_{B j}, Q^{2}, z\right)
$$

In der $\mathrm{e}^{+} \mathrm{e}^{-}$-Annihilation ist $\mathrm{Q}^{2} \equiv W^{2}$ und die beobachtete Abhängigkeit der $\mathrm{x}_{p}-$ Verteilungen von $\mathrm{W}$ mit den Erwartungen aufgrund der QCD-Korrekturen konsistent.

In der Lepton-Nukleon-Streuung wurden die erwarteten Effekte der Skalen- und Faktorisierungsbrechung schon in früheren Experimenten detailliert untersucht (z.B. [88],[78],[89],[90]). Die in [89] und [90] beobachteten Variationen der Fragmentationsfunktionen mit den Lepton-Produktionsvariablen sind mit QCD-Vorhersagen nicht inkonsistent. Allerdings erfordern Untersuchungen dieser Art in der LeptonNukleon-Streuung hochpräzise Datensätze (statistisch und systematisch), da es wegen der Korrelationen der kinematischen Variablen $\left(W^{2}=M_{p}^{2}+Q^{2}\left(\frac{1}{x_{B}}-1\right)\right)$ äußerst schwierig ist, beobachtete Variationen eindeutig einer Variablen zuzuordnen. Eine Möglichkeit, dieses Problem zu umgehen, besteht z.B. darin, Messungen bei verschiedenen Strahlenergien durchzuführen [18]. 
Die Skalenbrechung wurde in der $\mathrm{e}^{+} \mathrm{e}^{-}-$Vernichtung hingegen klar beobachtet (z.B. [91], [83]).

In dieser Analyse wird im Hinblick auf skalen- und faktorisierungsbrechende Effekte nur versucht, globale Variationen des gewichteten Mittels der Fragmentationsfunktionen festzustellen, da zwar einerseits der kinematisch zugängliche Bereich gegenüber früheren Lepton-Nukleon-Streuexperimenten erheblich erweitert ist andererseits die Datenstatistik präzise Untersuchungen limitiert. In Abbildung 6.8 sind die z-Verteilungen für a) drei $x_{B j}$-Intervalle und jeweils vier b) $\nu-$, c) $Q^{2}-$ und d) $W^{2}$-Intervalle dargestellt (wobei jeweils über die anderen kinematischen Variablen integriert wurde), in Abbildung 6.9 der Steigungsparameter b der einfachen Anpassung $e^{a+b z}$. Insbesondere in $W^{2}$ scheint ein klarer Trend $z u$ steiler werdenden Verteilungen mit zunehmendem $\mathrm{W}^{2}$ zu bestehen. In Abbildung 6.10 ist der Steigungsparameter für die $\mathrm{W}^{2}$-Abhängigkeit zusammen mit den entsprechenden Verteilungen aus den angeführten Annihilations-Experimenten dargestellt. Auffällig ist, daß ein deutlicher Unterschied zwischen den Steigungsparametern im gesamten ver-

gleichbaren $W^{2}-$ Bereich besteht. Darüberhinaus scheint die Änderung der Steigung mit $W^{2}$ in den $\mathrm{e}^{+} \mathrm{e}^{-}-$Vernichtungs- Experimenten ähnlich zu verlaufen wie in der $\mu-\mathrm{N}$-Streuung.

\subsection{Die Verteilungen des Transversalimpulses}

In Abbildung 6.11 ist die Verteilung des Quadrates des Transversalimpulses für geladene Hadronen dargestellt. Zum Vergleich sind Daten der EMC miteingetragen [92], [69]. Abbildung 6.12 zeigt die E665-Daten zusammen mit $\mathrm{P}_{T}^{2}$-Spektren aus der $\nu$-Nukleon-Streuung ([82], [93]). Man erkennt den typischen exponentiellen Abfall der Verteilung mit einem deutlichen Abflachen bei hohen $\mathrm{P}_{T}^{2}$.

Obwohl die Daten in unterschiedlichen kinematischen Bereichen und unterschiedlichen Prozessen genommen wurden, scheinen (wie im Fall der longitudinalen Variablen) die Verteilungen global recht gut übereinzustimmen (insbesondere die $\mu$ Nukleon-Experimente).

Ein Vergleich zu Daten aus der $\mathrm{e}^{+} \mathrm{e}^{-}$-Annihilation wird hier nicht vorgenommen, da der völlig unterschiedliche Anfangszustand eine Interpretation äußerst schwierig gestaltet:

Erstens ist in der Lepton-Nukleon-Streuung die Richtung des virtuellen Photons bekannt - in den $\mathrm{e}^{+} \mathrm{e}^{-}$-Annihilations-Experimenten wird eine entsprechende Referenzachse aus der Ereignistopologie gewonnen - und zweitens besitzen die Quarks im Nukleon einen primordialen Transversalimpuls $\left(\mathrm{k}_{T}\right)$ der beim $q \bar{q}$-Anfangszustand 

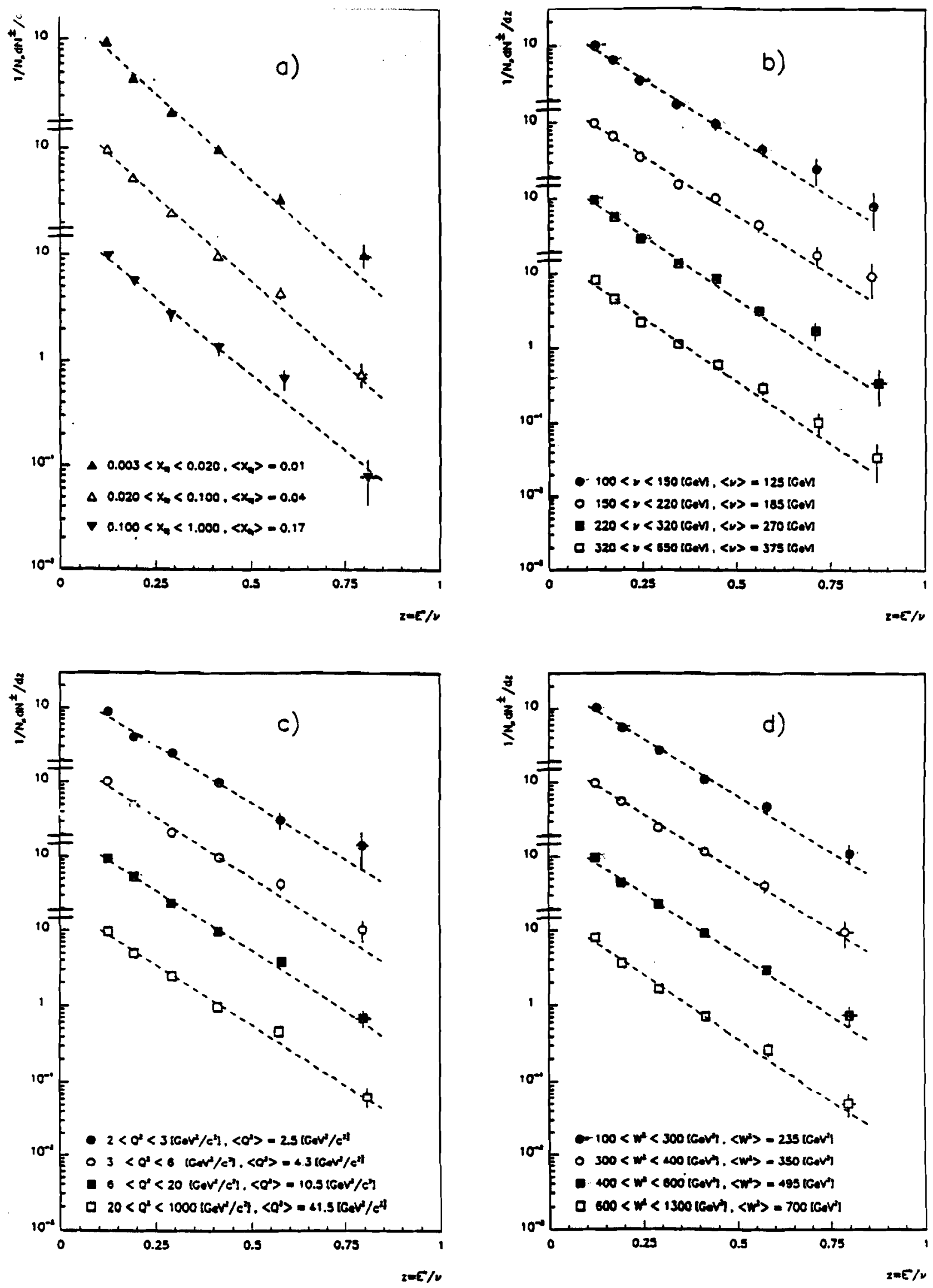

Abbildung 6.8: Z-Verteilungen für a) drei $x_{B_{j}}$, und jeweils vier b) $\nu-$, c) $Q^{2}-$ uncl d) $W^{2}$-Intervalle 

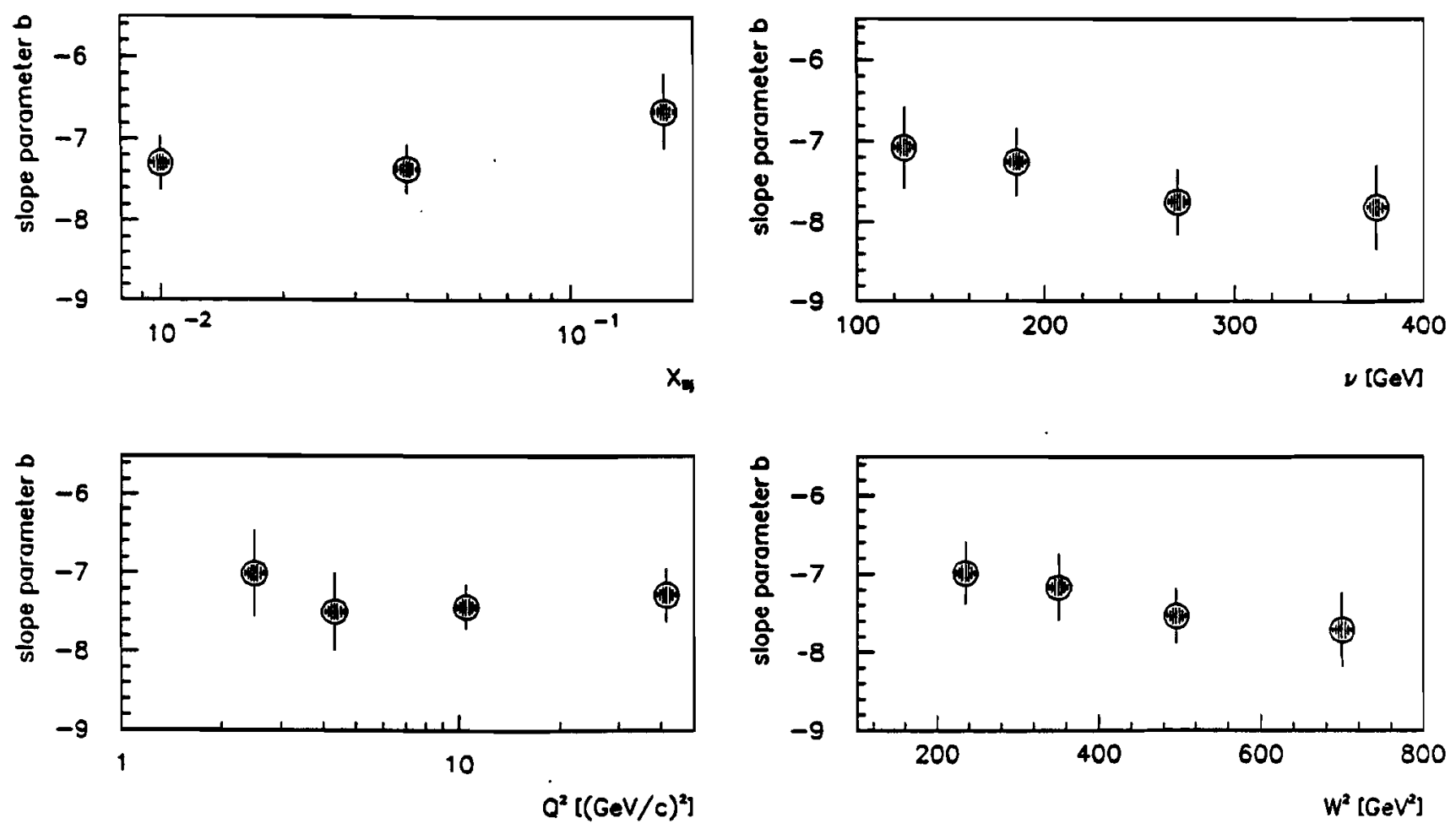

Abbildung 6.9: Steigungsparameter $\mathrm{b}$ der Anpassung $\mathrm{e}^{a+b z}$ an die z-Verteilungen gegen a) $x_{B j}$, b) $\nu$, c) $Q^{2}$ und d) $W^{2}$ 


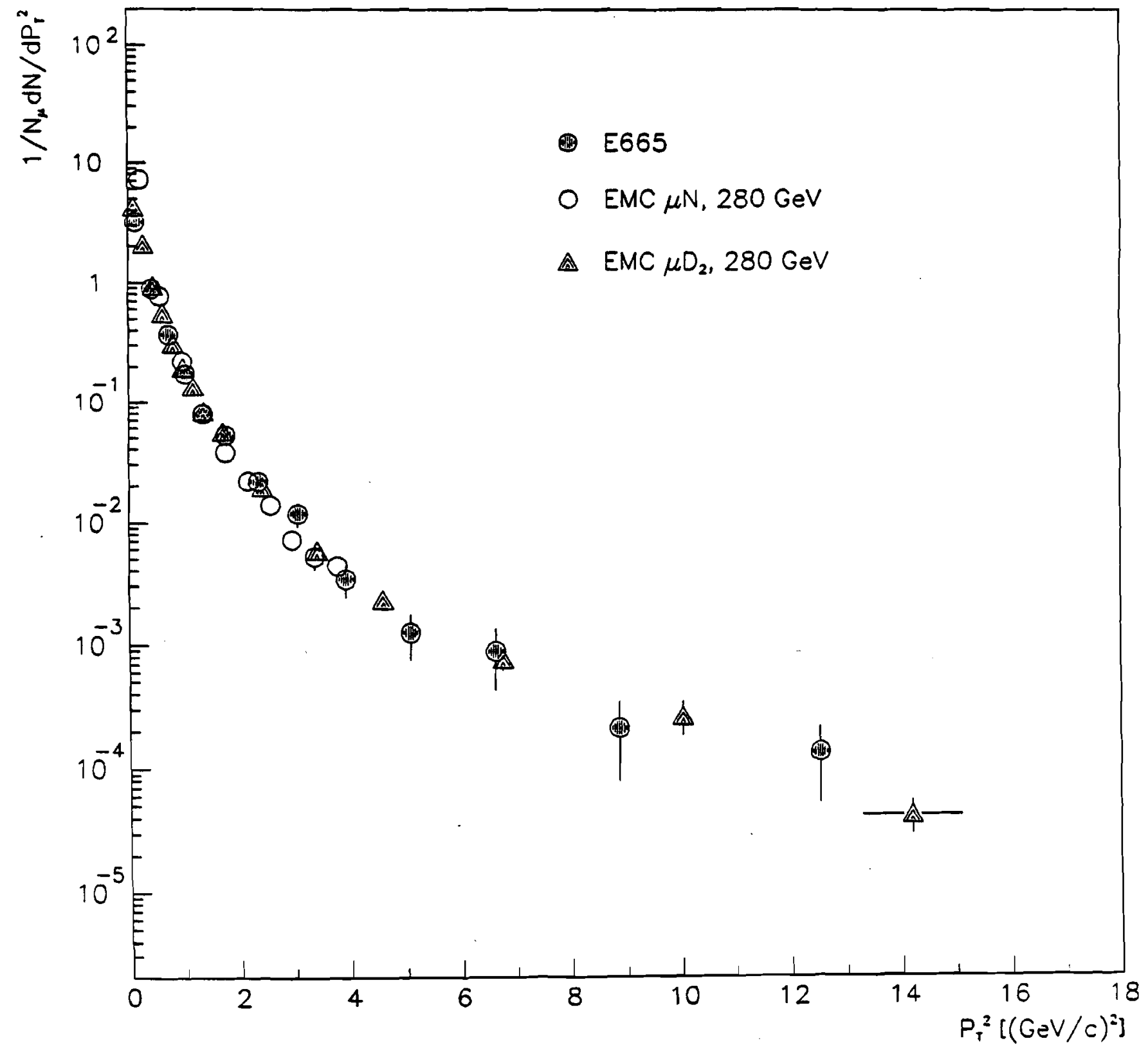

Abbildung 6.11: Normiertes differentielles $\mathrm{P}_{T}^{2}$-Spektrum der E665-Daten im Vergleich zu Messungen der EMC 
in den Annihilations-Experimenten keine Entsprechung findet. Ein detaillierter Vergleich, der diese Problematik berücksichtigt, ist in [85] zu finden.

In der Lepton-Nukleon-Streuung setzt sich der gesamte Transversalimpuls aus folgenden Beiträgen zusammen.

1. Dem primordialen Transversalimpuls der Partonen im Nukleon. Aufgrund der Unschärferelation erwartet man ein $\mathbf{k}_{T}$ von einigen hundert $\mathrm{MeV}\left(<k_{T}>\sim\right.$ $\frac{1}{R_{N_{\text {ucleon }}}}$ ).

2. Dem Anteil, der aufgrund harter QCD-Prozesse wie z.B. Gluon-Bremsstrahlung und Gluon-Photon-Fusion zum Transversalimpuls beiträgt. Dieser Anteil sollte proportional zum Produkt der Kopplungskonstanten $\alpha_{s}\left(Q^{2}\right)$ und $\mathrm{W}^{2}$ sein [94],[95].

3. Dem Beitrag, der aufgrund der Hadronisierung der Partonen nach dem Stoßprozeß zum gesamten $\mathrm{P}_{T}$ beiträgt.

Faßt man diese Beiträge in folgender Weise zusammen [96], so schreibt sich der gesamte mittlere Transversalimpuls zu:

$$
<P_{T}^{2}>=\left(<k_{T}^{2}>_{\text {primordial }}+<P_{T}^{2}>_{Q C D}\right) z^{2}+<P_{T}^{2}>_{\text {frag. }}
$$

Entsprechend dieser Vorhersagen erwartet man eine Abhängigkeit des mittleren Transversalimpulses von $\mathrm{z}^{2}$ und $\mathrm{W}^{2}$.

In Abbildung 6.13 ist der quadratische Transversalimpuls für drei verschiedene $\mathrm{W}^{2}-$ Bereiche dargestellt. Es ist ein klarer Trend für zunehmendes $P_{T}^{2}$ mit wachsendem $W^{2}$ erkennbar. Zum Vergleich sind wiederum Daten der EMC, die bei niedrigerem $\mathrm{W}^{2}$ liegen, miteingetragen [69]. Vergleichbare Daten für die beiden höheren $W^{2}-$ Intervalle liegen nicht vor, da E665 das bisher einzige Lepton-Nukleon-Streuexperiment ist, das in diesen kinematischen Bereich vordringen kann.

Die entsprechend den QCD-Effekten erwarteten Abhängigkeiten zeigen sich deutlich in Abbildung 6.14. Hier ist der mittlere Transversalimpuls gegen $\mathrm{W}^{2}$ für drei verschiedene $\mathrm{z}$-Intervalle aufgetragen. Der mittlere Transversalimpuls nimmt mit $\mathrm{W}^{2}$ $\mathrm{zu}^{9}$, insbesondere mit größer werdendem $\mathrm{z}$.

Die folgenden Abbildungen $6.15 \mathrm{a})$, b), c) zeigen die Verteilung des mittleren Transversalimpulses im Vergleich zu anderen Experimenten in den drei z-Intervallen (EMC:[9T]; $\left.\nu \mathrm{Ne}:[98] ; \nu \bar{\nu} \mathrm{D}_{2}:[99]\right)$. Auch hier zeigt sich im Rahmen der statistischen Fehler eine

\footnotetext{
${ }^{9} \mathrm{An}$ dieser Stelle sei bemerkt, daB für das z-Intervall $0.4<z<1.0$ Datenpunkte für $\mathrm{W}^{2}>$ $700 \mathrm{GeV}^{2}$ nicht gezeigt werden, da speziell in diesem Bereich neben der geringen Statistik die systematischen Unsicherheiten erheblich sind.
} 


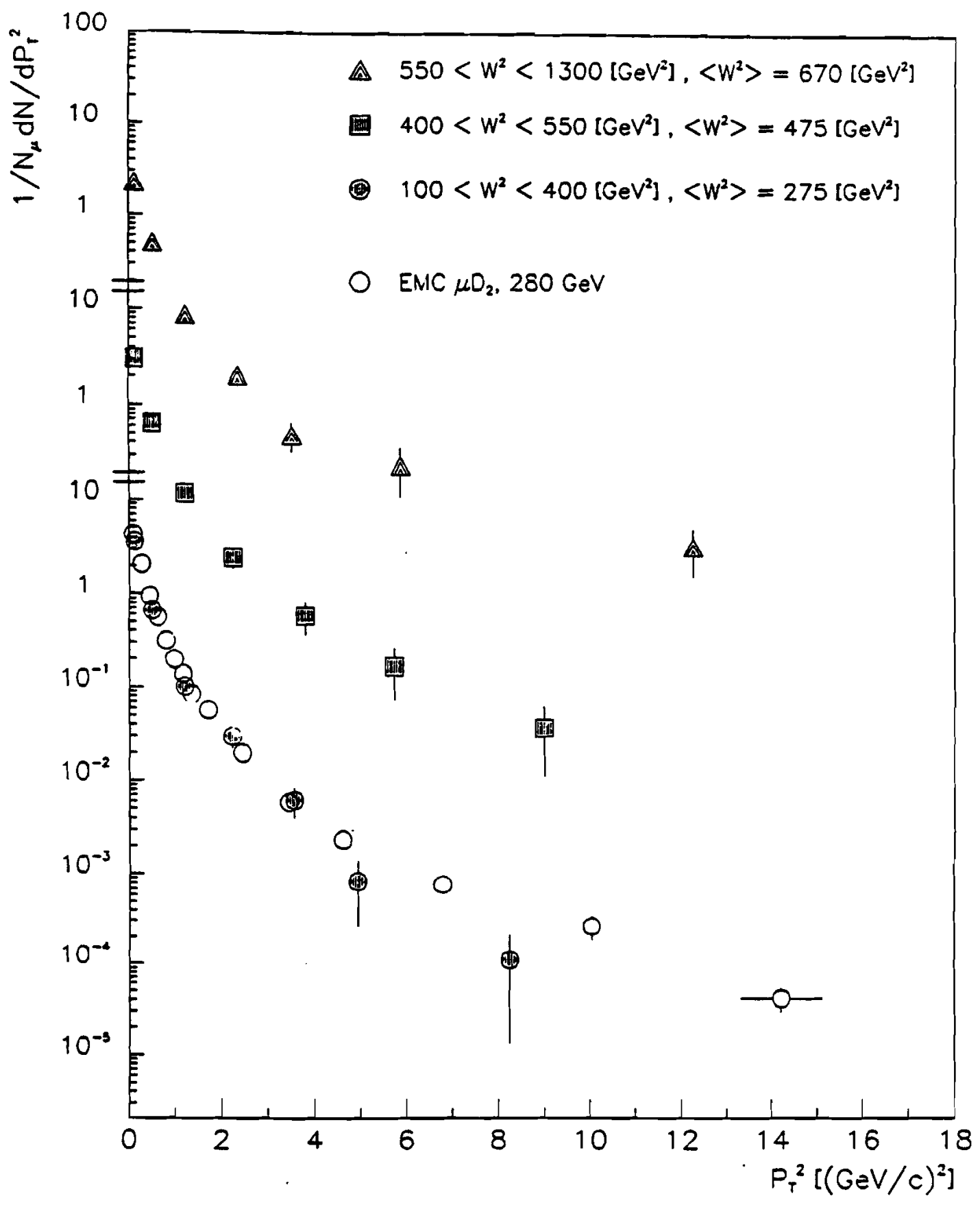

Abbildung 6.13: $\mathrm{P}_{T}^{2}$-Spektrum der E665-Daten in drei $\mathrm{W}^{2}$-Intervallen im Vergleich zu Messungen der EMC 


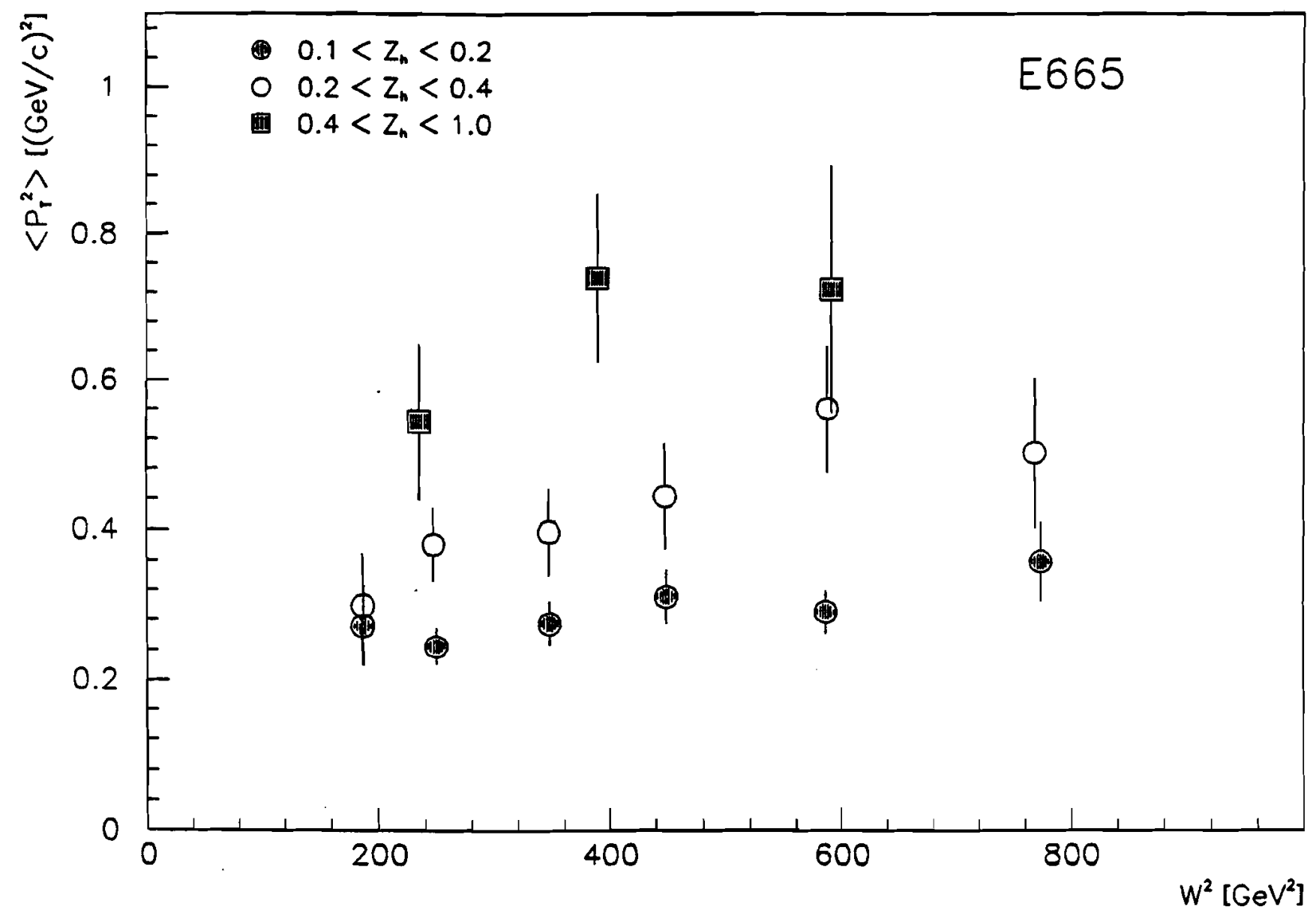

Abbildung 6.14: Mittleres $\mathrm{P}_{T}^{2}$ gegen $\mathrm{W}^{2}$ für drei verschiedene z-Intervalle 

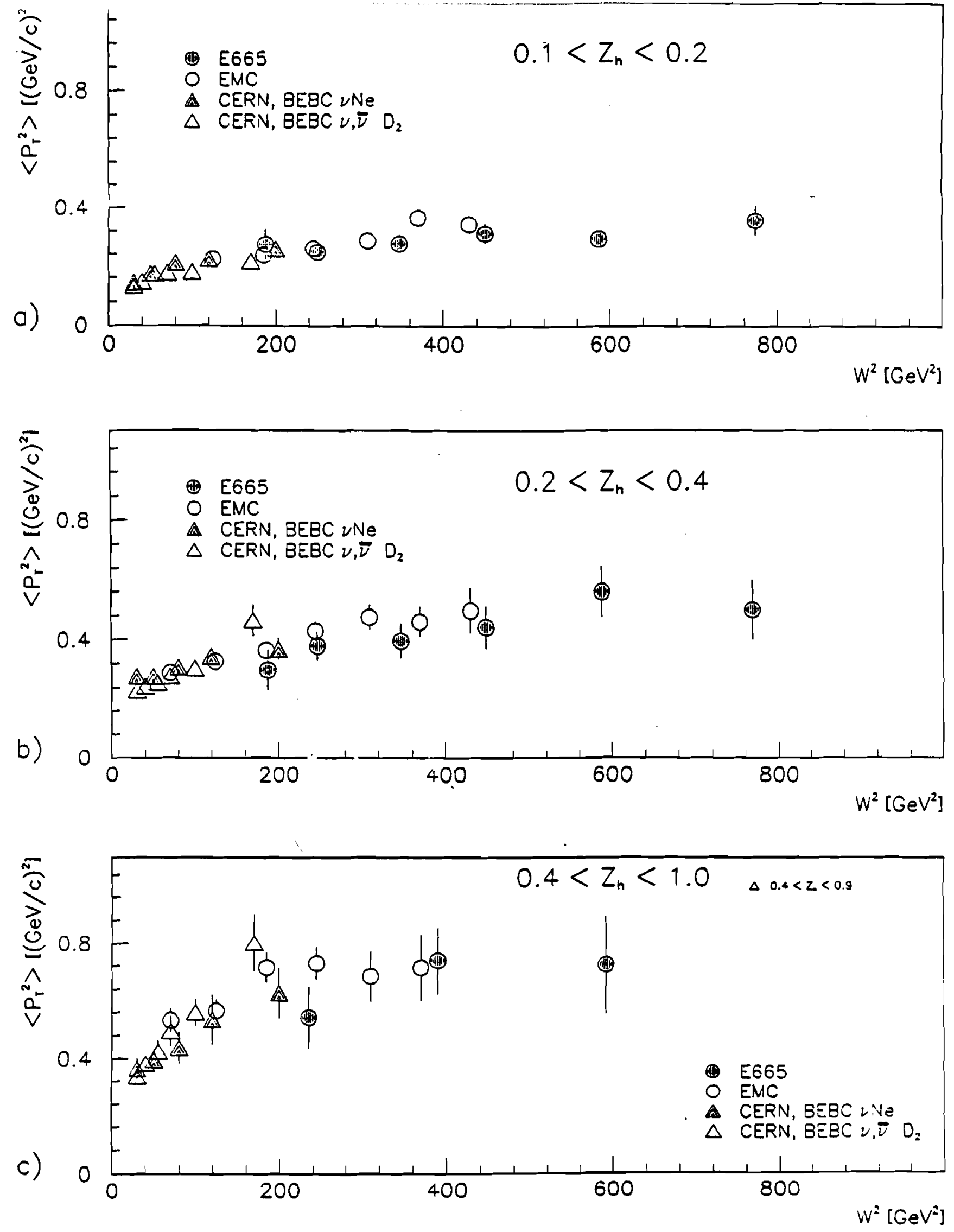

Abbildung 6.15: Mittleres $\mathrm{P}_{T}^{2}$ gegen $\mathrm{W}^{2}$ für a) $0.1<z<0.2$ b) $0.2<z<0.4$ c) $0.4<z<1.0$ im Vergleich zu Daten der EMC und Neutrino-Experimenten 
gute Übereinstimmung. Insbesondere scheint sich der Trend der Daten auch bei hohem $\mathrm{W}^{2}\left(W^{2}>400 \mathrm{GeV}^{2}\right)$ fortzusetzen.

In Abbildung 6.16 ist $\left\langle P_{T}^{2}>\right.$ gegen $\mathrm{z}^{2}$ aufgetragen. Das typische Ansteigen des mittleren $P_{T}^{2}$ mit $z^{2}$ ist deutlich ('Seagull-plot'). In a) sind die Daten aus diesem Experiment zusammen mit Messungen der EMC gezeigt (EMC $\mu \mathrm{N}:[69]$; EMC $\mu \mathrm{p}:$ [97]). Die Daten stimmen gut überein und spiegeln qualitativ den erwarteten Verlauf der QCD-Vorhersagen wieder. Der Vergleich der E665-Daten mit den $<P_{T}^{2}>$-Spektren gemessen in $\nu \bar{\nu} \mathrm{N}$-Streuexperimenten zeigt deutliche Unterschiede (Abb.:6.16 b), $\nu \bar{\nu} \mathrm{D}_{2}:[99] ; \nu \mathrm{P}:[100] ; \nu \bar{\nu} \mathrm{N}:[82]$ ), allerdings wurden die Daten der Neutrino-Experimente im allgemeinen bei deutlich niedrigeren Energien genommen. Der Trend der Daten wird jedoch auch von den $\nu$-Experimenten wiedergegeben.

Neben der qualitativen Übereinstimmung der Daten mit den theoretischen Vorhersagen werden im folgenden Abschnitt die Daten mit verschiedenen Modellen quantitativ verglichen.

\subsection{Vergleich der Daten mit Modellvorhersagen}

Unter der Vielzahl der möglichen Modelle zum Datenvergleich fiel die Auswahl auf drei verschiedene Versionen des Lund-Modells [101].

1. Lund 4.3 (Lepto $4.3+$ Jetset 4.3 )

2. Lund 6.3 (Lepto $5.2+$ Jetset 6.3) ME

3. Lund 6.3 (Lepto $5.2+$ Jetset 6.3) PA

Die ältere Version 4.3 beschreibt gut die Daten der EMC [27], [102]. Allerdings erscheint die Behandlung niederenergetischer Gluonen (soft gluons) in diesem Modell problematisch. In der Version 6.3 besteht die Möglichkeit in der perturbativen Phase der Hadronisierung entweder eine exakte Berechnung der Matrixelemente in erster Ordnung $\alpha_{s}$ durchzuführen (mit ME gekennzeichnete Version, bzw. MX in den Abbildungen) oder aber partonische Schauer zu erzeugen (mit PA gekennzeichnete Version), deren Entwicklung in der 'leading log' Approximation ausgeführt wird. Für den Vergleich zu den Daten wurden die Standard-Parameter der Lund-Programme übernommen.

In Abbildung 6.17 sind die Modellkurven zusammen mit den Daten für den mittleren Transversalimpuls in Abhängigkeit von der invarianten Masse in drei verschiedenen z-Intervallen dargestellt. 

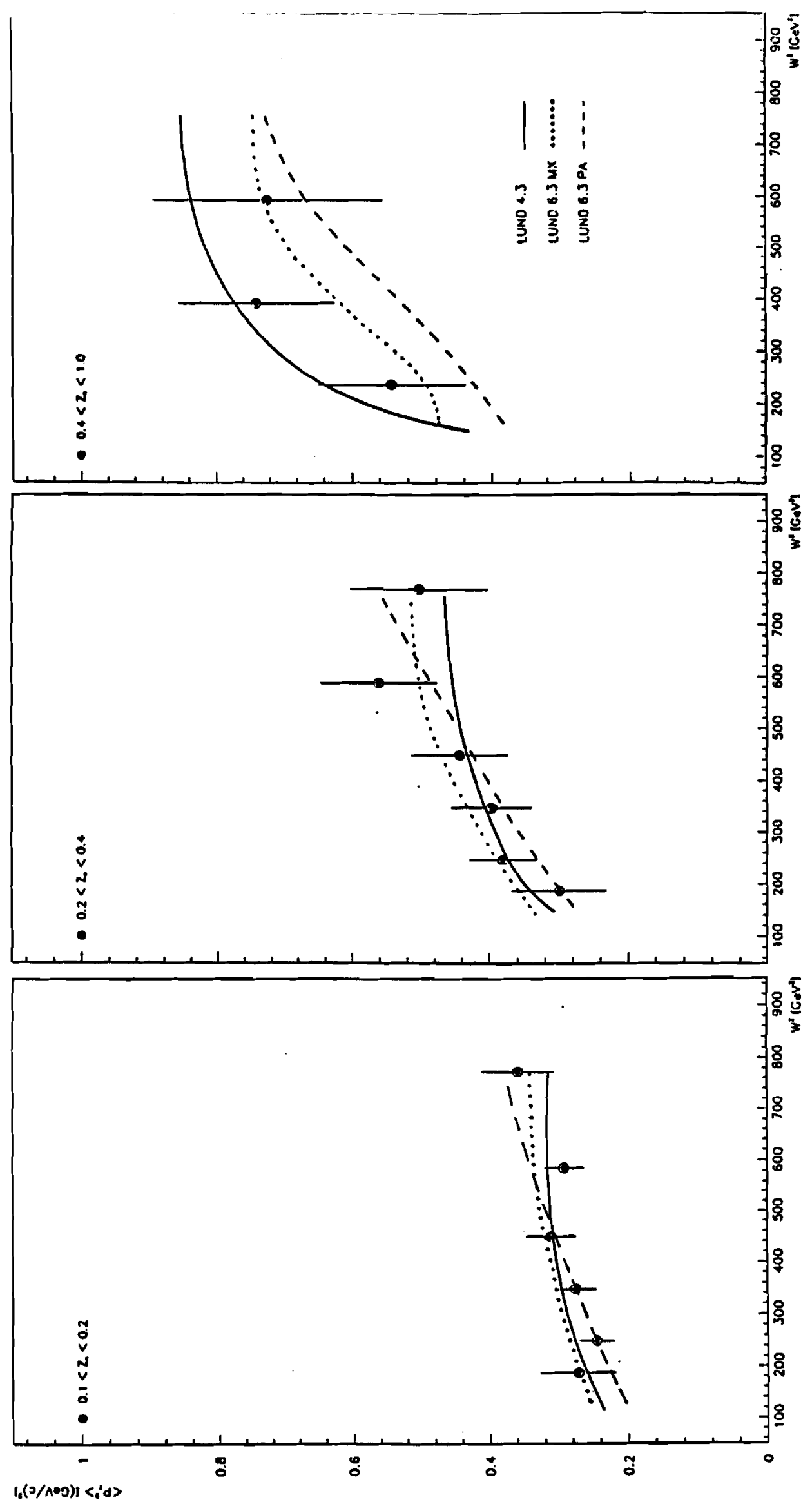

Abbildung 6.17: Mittleres $\mathrm{P}_{T}^{2}$ gegen $\mathrm{W}^{2}$ im Vergleich zu verschiedenen Versionen des Lund-Modells. 
Insbesondere im höchsten z-Bereich unterscheiden sich die Modellversionen deutlich. Der mittlere Transversalimpuls des Lund-Modells Version 4.3 liegt allgemein ca. $25 \%$ höher als in der Partonschauer-Variante. Allerdings sind die Daten aufgrund der limitierten Statistik mit allen Versionen verträglich.

Ein deutlich unterschiedliches Bild ergibt sich für den Vergleich in Abbildung 6.18 $\left(<P_{T}^{2}\right\rangle$ versus $z^{2}$ ). Die Vorhersagen des Lund-Modells Version 4.3 zeigt ein kontinuierliches Ansteigen des mittleren Transversalimpulses mit $\mathbf{z}^{2}$, wohingegen die Modellversionen 6.3 ihr maximales mittleres $\mathrm{P}_{T}^{2}$ bei einem $\mathrm{z}$ von $\sim 0.4$ erreichen und anschließend mit zunehmendem $\mathrm{z}$ wieder abfallen. Obwohl der statistische Fehler der Daten erheblich ist, erscheinen sie mit den aktuelleren Versionen des Lund-Modells besser verträglich als mit Version 4.3. Der systematische Fehler des mittleren $\mathrm{P}_{T}^{2}$ bei $z^{2} \simeq 0.45(0.75)$ wird auf oben beschriebene Weise abgeschätzt. Damit ergibt sich: $\left\langle P_{T}^{2}\right\rangle=0.708 \pm 0.161_{\text {,tat. }} \pm 0.113_{\text {,yot. }}(0.508 \pm 0.221 \pm 0.299)$ und bei quadratischer Addition des statistischen und systematischen Fehlers eine Abweichung von der Modellvorhersage (Version 4.3) von 1.1 $\sigma(1.7 \sigma)$. 


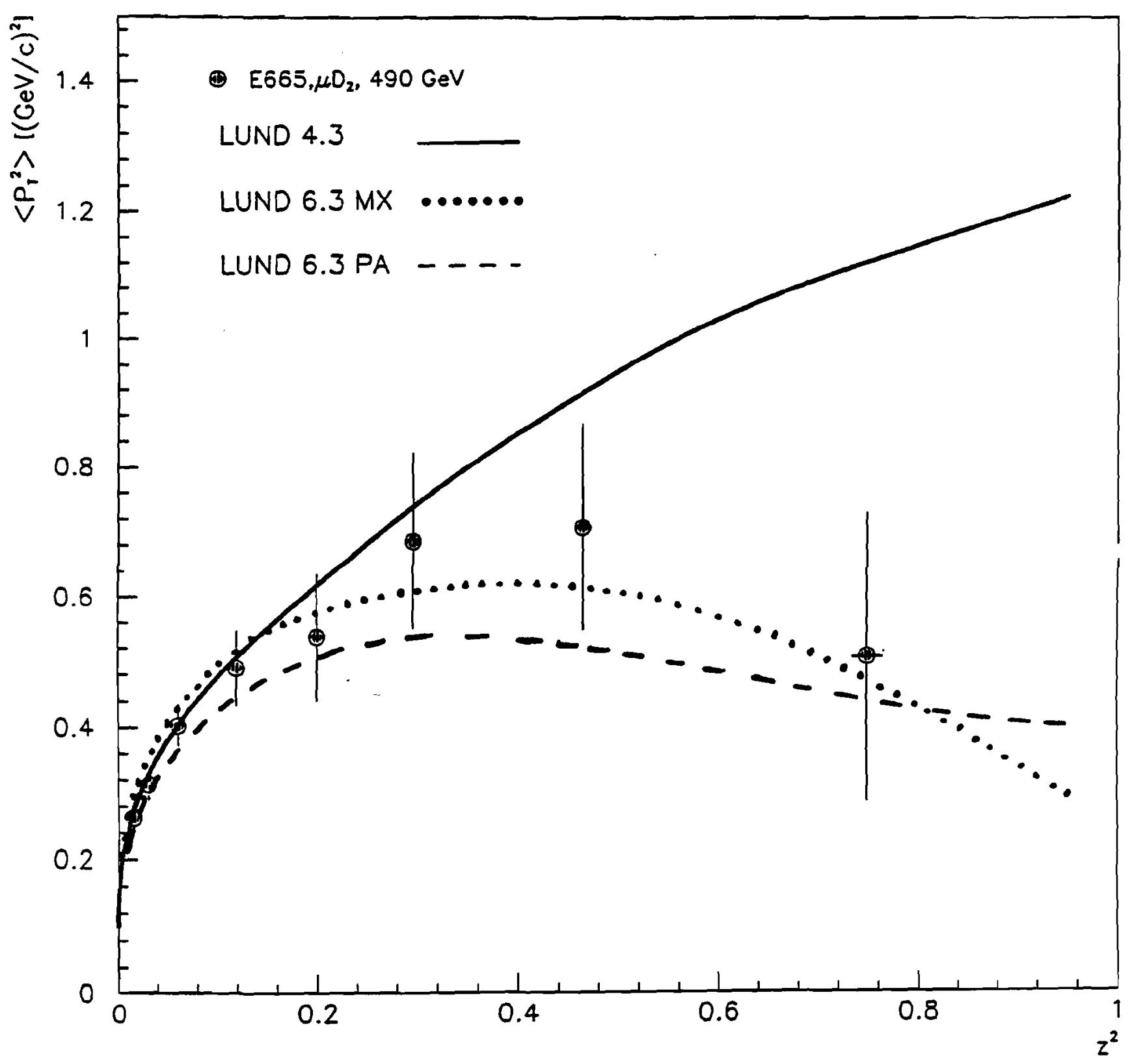

Abbildung 6.18: Mittleres $\mathrm{P}_{T}^{2}$ gegen $\mathrm{z}^{2}$ im Vergleich zu verschiedenen Versionen des Lund-Modells. 


\section{Zusammenfassung}

In der Periode vom Juni 1987 - Februar 1988 wurden die ersten Daten mit dem neuen Spektrometer der E665-Kollaboration am Fermilab erfaßt. 490-GeV-Myonen streuten tiefinelastisch an Wasserstoff, Deuterium und Xenon, 100-GeV-Myonen an Deuterium und Xenon. In diesem Experiment konnte ein kinematischer Bereich erschlossen werden, in dem bisher keine in der Leptoproduktion gewonnenen Daten vorlagen.

In dieser Analyse wurden Daten der 490-GeV-Myon-Deuterium-Streuung verwendet und unter Aspekten des hadronischen Endzustandes studiert.

Die Verteilungen der longitudinalen Hadronvariablen $z_{h}$ zeigen eine leichte Abhängigkeit von den Myonvariablen; insbesondere von der invarianten Masse W. Die $z_{h}$-Verteilung wird mit zunehmender invarianter Masse steiler. Dieses Verhalten wird aufgrund von QCD-Effekten erwartet.

Die über den gesamten kinematischen Bereich integrierten Verteilungen stimmen gut mit Daten aus anderen Myon-Streuexperimenten überein. Ein Vergleich zu Neutrino-Streudaten (CC-Reaktionen) stellt sich nicht so gut dar. Allerdings wurden diese Daten im allgemeinen bei deutlich niedrigeren Energien genommen. Im Vergleich $z u x_{p}$-Verteilungen aus $\mathrm{e}^{+} \mathrm{e}^{-}-$Annihilations-Experimenten zeigt sich, daß bei gleicher invarianter Masse die $x_{p}$-Verteilungen generell steiler sind als die vergleichbaren $z_{h}$-Verteilungen in der Myoproduktion. Die Änderung der Steigungen mit der invarianten Masse scheint jedoch in beiden Produktionsprozessen gleich zu sein.

Die Verteilungen der transversalen Hadronvariablen $\mathrm{P}_{T}^{2}$ und $\left\langle P_{T}^{2}\right\rangle$ zeigen eine deutliche $A b h a ̈ n g i g k e i t$ von $W^{2}$ und $z_{h}$, wie sie im Rahmen der QCD erwartet wird.

Die Daten stimmen recht gut mit Verteilungen aus anderen Leptoproduktionsexperimenten überein, insbesondere mit Daten aus der Myoproduktion.

Im quantitativen Vergleich wurden die mittleren Transversalimpulsverteilungen mit verschiedenen Versionen des Lund-Modells konfrontiert.

In der Abhängigkeit des mittleren $\mathrm{P}_{T}^{2}$ von $\mathrm{W}^{2}$ treten in den Modellvorhersagen deutliche Unterschiede bei hohen $z_{h}$ auf. Aufgrund der limitierten Datenstatistik kann jedoch kein Modell bevorzugt werden.

Die Abhängigkeit des mittleren Transversalimpulses von $z^{2}$ divergiert für die ver- 
schiedenen Modellversionen bei $z^{2} \sim 0.4$. Das Lund-Modell Version 4.3 zeigt ein kontinuierliches Ansteigen von $\left\langle P_{T}^{2}\right\rangle$ mit $z^{2}$, wohingegen die aktuelleren Versionen ein Abfallen des mittleren Transversalimpulses vorhersagen. Die Daten scheinen auch unter Berücksichtigung der statistischen und systematischen Unsicherheiten den abfallenden Trend des Lund-Modells Version 6.3 in dieser Verteilung zu bevorzugen.

Diese Arbeit und die zahlreichen zugrundeliegenden Studien zeigen, daß ein hochwertiger Datensatz mit dem neuen Spektrometer der E665-Kollaboration gewonnen wurde. Zur Zeit wird am Fermilab mit diesem Spektrometer die zweite Datenperiode durchgeführt. Eine Zusammenfassung der gesamten Datenmenge wird einen Datensatz mit hoher Statistik ergeben und damit genauere Untersuchungen mit Hinblick auf die in dieser Analyse aufgeworfenen Fragestellungen zulassen. Insbesondere wird ein detaillierter Vergleich zu $\mathrm{e}^{+} \mathrm{e}^{-}$-Experimenten bei Schwerpunktenergien von bis zu $30 \mathrm{GeV}$ möglich sein. 


\section{Danksagung}

Mein Dank gilt allen, die zu einer erfolgreichen Durchführung des Experimentes 665 beigetragen haben.

Herr Prof. Dr. J. Drees regte diese Arbeit an und ermöglichte mir den dreijährigen Aufenthalt am Fermilab in den Vereinigten Staaten. Während meiner fünfjährigen Tätigkeit an diesem Projekt war ich mir seiner Hilfsbereitschaft und seinem fachkundigen Rat stets sicher. An dieser Stelle möchte ich meinen aufrichtigen Dank hierfür aussprechen.

Herrn Dr. Helmut Braun und Herrn Dr. Manfred Pötsch danke ich für die unzähligen Diskussionen und Anregungen, die in all den Jahren zu einem aktiven und effektiven Arbeitsklima beitrugen. Dank auch für die kritische Durchsicht dieser Arbeit.

Herrn Dr. Hugh E. Montgomery danke ich für seinen motivierenden Optimismus und die vielfältigen Impulse und fachlichen Anleitungen, die wesentlich zu einem produktiven Arbeiten während meines Aufenthaltes am FNAL beigetragen haben.

Unter all den Kollegen am Fermilab möchte ich mich besonders bei Silhacene Aïd und Dr. Erik J. Ramberg bedanken. Sie waren mir nicht nur bei all den Problemen im Laboralltag, sondern auch, insbesondere während meiner Anfangszeit in den Vereinigten Staaten, im US-Alltag behilflich - Thanks, guys.

Frau Voswinkel danke ich für das sorgfältige Anfertigen der Feynman-Graphen.

Last but certainly not least gilt mein Dank meiner Familie und ganz besonders meiner Ehefrau Petra, die sicherlich nicht nur durch das mühevolle Beseitigen meiner Tippfehler zu einem Gelingen dieser Arbeit beigetragen hat. 


\section{A Tabellierte Daten der differentiellen Multiplizitäten und Verteilungen des mittleren Transversalimpulses}

\begin{tabular}{|c|c|c|c|}
\hline$z_{h}$-Intervall & $\left\langle z_{h}\right\rangle$ & $\frac{1}{N_{\mu}} \frac{d N^{+}}{d z_{h}}$ & stat. Fehler \\
\hline $0.10, \quad 0.20$ & 0.142 & 4.073 & \pm 0.168 \\
\hline,$\quad 0.35$ & 0.263 & 1.470 & $\pm 8.13710^{-2}$ \\
\hline,$\quad 0.55$ & 0.433 & 0.496 & $\pm 4.14810^{-2}$ \\
\hline $0.55,1.00$ & 0.678 & $8.70510^{-2}$ & $\pm 1.18110^{-2}$ \\
\hline
\end{tabular}

Tabelle A1: z-Verteilung für positive Hadronen

\begin{tabular}{|c|c|c|c|}
\hline$z_{h}$-Intervall & $\left\langle z_{h}\right\rangle$ & $\frac{1}{N_{\mu}} \frac{d N^{-}}{d z_{h}}$ & stat. Fehler \\
\hline $0.10, \quad 0.20$ & 0.143 & 3.754 & \pm 0.159 \\
\hline 0.20, & 39 & 1.280 & $\pm 7.59010^{-2}$ \\
\hline 0.55 & 0.433 & 0.407 & $\pm 3.87210^{-2}$ \\
\hline 1.00 & 0.674 & $8.09610^{-2}$ & $\pm 1.31210^{-2}$ \\
\hline
\end{tabular}

Tabelle A2: $z$-Verteilung für negative Hadronen

\begin{tabular}{|c|c|c|c|c|}
\hline \multicolumn{2}{|c|}{$z_{h}$-Intervall } & $\left\langle z_{h}\right\rangle$ & $\frac{1}{N_{u}} \frac{d N}{d z_{k}}$ & stat. Fehler \\
\hline 0.10 & 0.15 & 0.123 & 9.560 & \pm 0.357 \\
\hline 0.15 & 0.20 & 0.173 & 6.071 & \pm 0.293 \\
\hline 0.20 & 0.25 & 0.223 & 3.587 & \pm 0.214 \\
\hline 0.25 & 0.30 & 0.273 & 2.797 & \pm 0.200 \\
\hline 0.30 & 0.35 & 0.324 & 1.889 & \pm 0.162 \\
\hline 0.35 & 0.40 & 0.374 & 1.197 & \pm 0.123 \\
\hline 0.40 & 0.45 & 0.425 & 1.144 & \pm 0.140 \\
\hline 0.45 & 0.50 & 0.474 & 0.741 & $\pm 9.99310^{-2}$ \\
\hline 0.50 & 0.55 & 0.523 & 0.579 & $\pm 9.44310^{-2}$ \\
\hline 0.55 & 0.60 & 0.574 & 0.422 & $\pm 7.91910^{-2}$ \\
\hline 0.60 & 0.70 & 0.647 & 0.273 & $\pm 4.89010^{-2}$ \\
\hline 0.70 & 0.80 & 0.746 & 0.138 & $\pm 3.05410^{-2}$ \\
\hline 0.80 & 0.90 & 0.840 & 0.118 & $\pm 3.64510^{-2}$ \\
\hline 0.90 & 1.00 & 0.935 & $2.26910^{-2}$ & $\pm 1.41610^{-2}$ \\
\hline
\end{tabular}

Tabelle A3: z-Verteilung für geladene Hadronen 


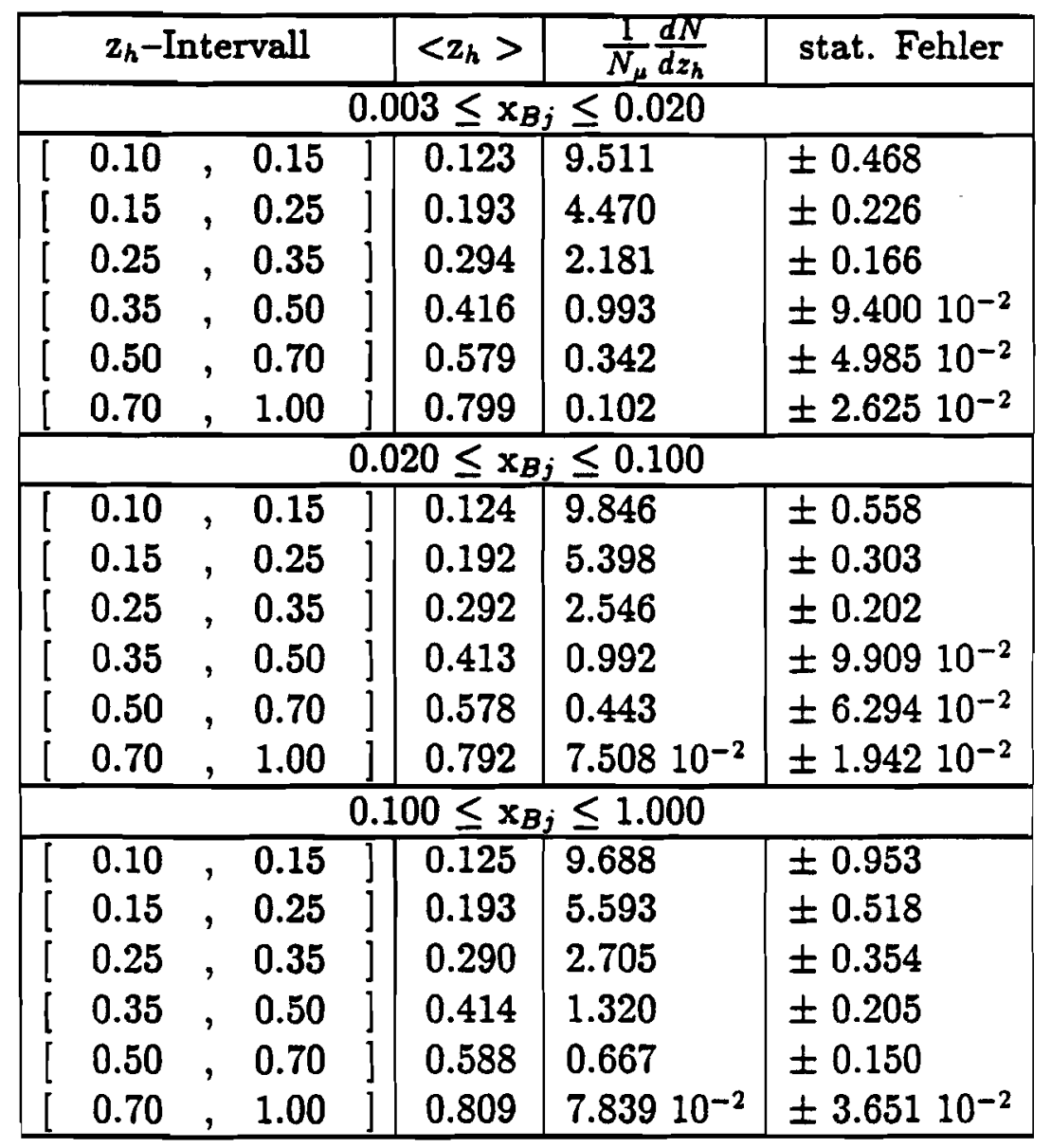

Tabelle A4: $z$-Verteilung für geladene Hadronen in drei verschiedenen $x_{B j}$-Intervallen 


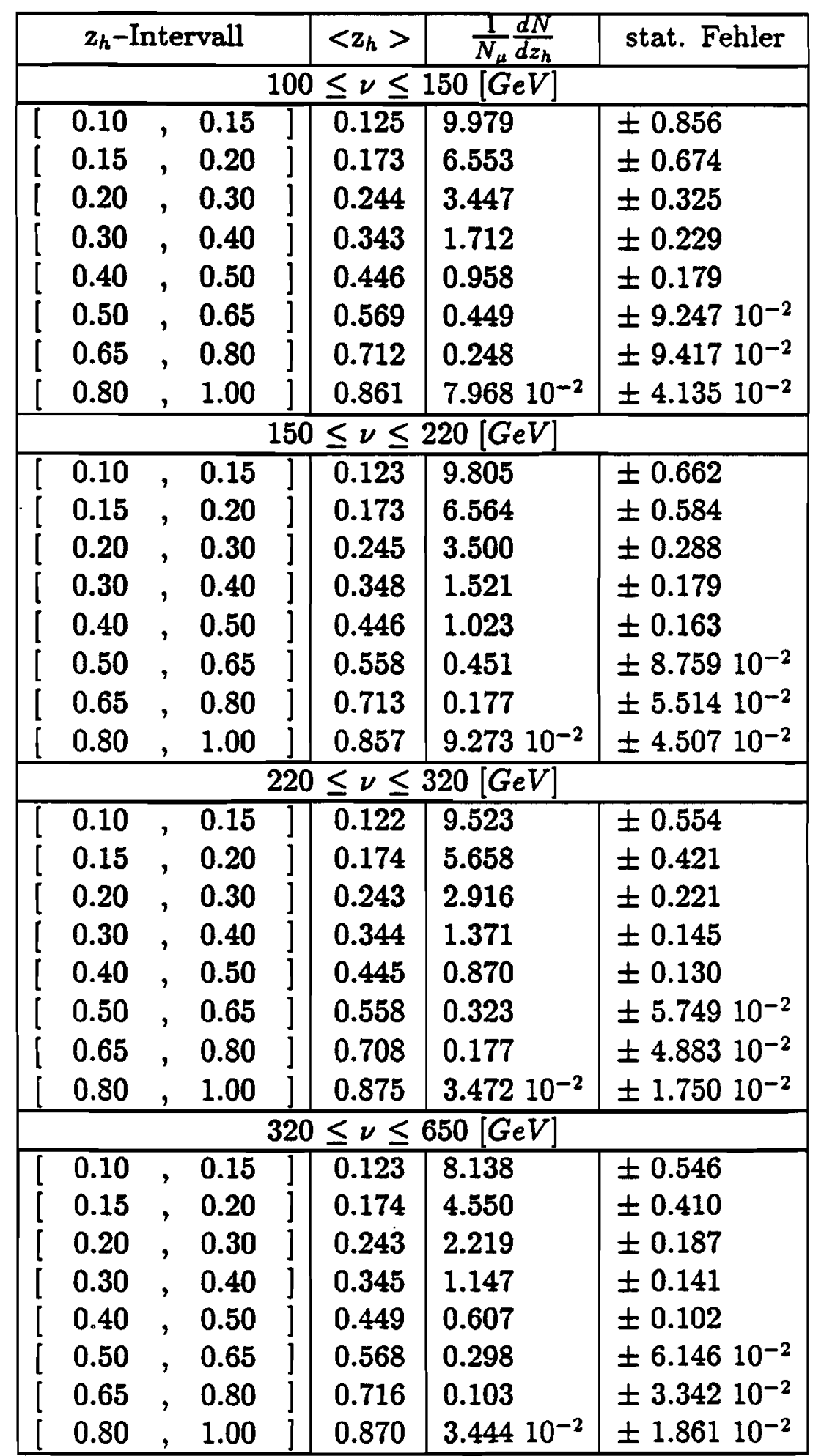

Tabelle A5: z-Verteilung für geladene Hadronen in vier verschiedenen $\nu$-Interrallen 


\begin{tabular}{|c|c|c|c|c|}
\hline \multicolumn{2}{|c|}{$z_{h}$-Intervall } & $\left\langle z_{h}\right\rangle$ & $\frac{1}{N_{\mu}} \frac{d N}{d z_{h}}$ & stat. Fehler \\
\hline \multicolumn{5}{|c|}{$2 \leq \mathrm{Q}^{2} \leq 3\left[(\mathrm{GeV} / \mathrm{c})^{2}\right]$} \\
\hline 0.1 &,$\quad 0.15$ & 0.123 & 8.936 & \pm 0.785 \\
\hline 0.1 &,$\quad 0.25$ & 0.192 & 4.024 & \pm 0.357 \\
\hline 0.2 &,$\quad 0.35$ & 0.294 & 2.445 & \pm 0.335 \\
\hline 0.3 &,$\quad 0.50$ & 0.415 & 0.999 & \pm 0.161 \\
\hline 0.5 &,$\quad 0.70$ & 0.578 & 0.312 & $\pm 7.98410^{-2}$ \\
\hline 0.7 &,$\quad 1.00$ & 0.795 & 0.143 & $\pm 7.48510^{-2}$ \\
\hline \multicolumn{5}{|c|}{$3 \leq \mathrm{Q}^{2} \leq 6\left[(\mathrm{GeV} / \mathrm{c})^{2}\right]$} \\
\hline 0.1 & 0.15 & 0.123 & 10.151 & \pm 0.666 \\
\hline 0.1 &,$\quad 0.25$ & 0.194 & 4.918 & \pm 0.322 \\
\hline 0.2 & 0.35 & 0.294 & 2.113 & \pm 0.211 \\
\hline 0.3 & 0.50 & 0.418 & 0.970 & \pm 0.121 \\
\hline 0.5 & 0.70 & 0.580 & 0.430 & $\pm 7.99010^{-2}$ \\
\hline 0.7 & 1.00 & 0.797 & 0.103 & $\pm 3.42010^{-2}$ \\
\hline \multicolumn{5}{|c|}{$6 \leq \mathrm{Q}^{2} \leq 20\left[(\mathrm{GeV} / \mathrm{c})^{2}\right]$} \\
\hline 0.1 &, 0.15 & 0.123 & 9.374 & \pm 0.494 \\
\hline 0.1 &,$\quad 0.25$ & 0.192 & 5.381 & \pm 0.282 \\
\hline 0.2 & , $\quad 0.35$ & 0.292 & 2.367 & \pm 0.176 \\
\hline 0.3 &,$\quad 0.50$ & 0.413 & 0.977 & $\pm 9.36710^{-2}$ \\
\hline 0.5 &,$\quad 0.70$ & 0.581 & 0.382 & $\pm 5.35610^{-2}$ \\
\hline 0.7 &,$\quad 1.00$ & 0.797 & $6.86110^{-2}$ & $\pm 1.73610^{-2}$ \\
\hline \multicolumn{5}{|c|}{$20 \leq \mathrm{Q}^{2} \leq 1000\left[(\mathrm{GeV} / \mathrm{c})^{2}\right]$} \\
\hline 0.1 &,$\quad 0.15$ & 0.124 & 9.648 & \pm 0.601 \\
\hline 0.1 &,$\quad 0.25$ & 0.193 & 4.921 & \pm 0.301 \\
\hline 0.2 & 0.35 & 0.293 & 2.448 & \pm 0.216 \\
\hline 0.3 &,$\quad 0.50$ & 0.412 & 0.967 & \pm 0.106 \\
\hline 0.5 &,$\quad 0.70$ & 0.573 & 0.466 & $\pm 6.85110^{-2}$ \\
\hline 0.7 & , 1.00 & 0.809 & $6.47010^{-2}$ & $\pm 1.95810^{-2}$ \\
\hline
\end{tabular}

Tabelle A6: $z$-Verteilung für geladene Hadronen in vier verschiedenen $\mathrm{Q}^{2}$-Intervallen 


\begin{tabular}{|c|c|c|c|c|}
\hline \multicolumn{2}{|c|}{$z_{h}$-Intervall } & $\left\langle z_{h}\right\rangle$ & $\frac{1}{N_{u}} \frac{d N}{d z_{h}}$ & stat. Fehler \\
\hline \multicolumn{5}{|c|}{$100 \leq \mathrm{W}^{2} \leq 300\left[\mathrm{GeV}^{2}\right]$} \\
\hline 0.10 & 0.15 & 0.125 & 10.187 & \pm 0.768 \\
\hline 0.15 & , 0.25 & 0.194 & 5.423 & \pm 0.381 \\
\hline 0.25 &,$\quad 0.35$ & 0.293 & 2.693 & \pm 0.269 \\
\hline 0.35 &,$\quad 0.50$ & 0.414 & 1.105 & \pm 0.132 \\
\hline 0.50 &,$\quad 0.70$ & 0.580 & 0.473 & $\pm 8.24910^{-2}$ \\
\hline 0.70 &,$\quad 1.00$ & 0.802 & 0.110 & $\pm 3.29010^{-2}$ \\
\hline \multicolumn{5}{|c|}{$300 \leq \mathrm{W}^{2} \leq 400\left[\mathrm{GeV}^{2}\right]$} \\
\hline 0.10 & 0.15 & 0.123 & 9.815 & \pm 0.761 \\
\hline 0.15 & 0.25 & 0.193 & 5.547 & \pm 0.436 \\
\hline 0.25 & 0.35 & 0.292 & 2.464 & \pm 0.283 \\
\hline 0.35 & 0.50 & 0.415 & 1.171 & \pm 0.163 \\
\hline 0.50 & 0.70 & 0.575 & 0.403 & $\pm 8.30410^{-2}$ \\
\hline 0.70 & 1.00 & 0.789 & $9.36910^{-2}$ & $\pm 3.68410^{-2}$ \\
\hline \multicolumn{5}{|c|}{$400 \leq \mathrm{W}^{2} \leq 600\left[\mathrm{GeV}^{2}\right]$} \\
\hline 0.10 &, 0.15 & 0.123 & 9.647 & \pm 0.549 \\
\hline 0.15 &,$\quad 0.25$ & 0.191 & 4.338 & \pm 0.254 \\
\hline 0.25 &,$\quad 0.35$ & 0.294 & 2.234 & \pm 0.195 \\
\hline 0.35 & , 0.50 & 0.415 & 0.907 & $\pm 9.96610^{-2}$ \\
\hline 0.50 &,$\quad 0.70$ & 0.579 & 0.293 & $\pm 4.72610^{-2}$ \\
\hline 0.70 & , 1.00 & 0.800 & $7.29910^{-2}$ & $\pm 2.12210^{-2}$ \\
\hline \multicolumn{5}{|c|}{$600 \leq \mathrm{W}^{2} \leq 1300\left[\mathrm{GeV}^{2}\right]$} \\
\hline 0.10 &, 0.15 & 0.123 & 7.888 & \pm 0.555 \\
\hline 0.15 & 0.25 & 0.193 & 3.551 & \pm 0.257 \\
\hline 0.25 & 0.35 & 0.293 & 1.616 & \pm 0.173 \\
\hline 0.35 & 0.50 & 0.417 & 0.708 & $\pm 9.40910^{-2}$ \\
\hline 0.50 & 0.70 & 0.583 & 0.266 & $\pm 5.42210^{-2}$ \\
\hline 0.70 &,$\quad 1.00$ & 0.797 & $4.93110^{-2}$ & $\pm 1.75310^{-2}$ \\
\hline
\end{tabular}

Tabelle A7: $\mathrm{z}$-Verteilung für geladene Hadronen in vier verschiedenen $\mathrm{W}^{2}$-Intervallen 


\begin{tabular}{|c|c|c|c|c|}
\hline $\begin{array}{r}\mathrm{P}_{T}^{2}-\mathrm{In} \\
{[(\mathrm{Ge})}\end{array}$ & $\begin{array}{l}\text { atervall } \\
\left.V / c)^{2}\right]\end{array}$ & $\begin{array}{c}\left\langle\mathrm{P}_{T}^{2}\right\rangle \\
{\left[(\mathrm{GeV} / \mathrm{c})^{2}\right]}\end{array}$ & $\begin{array}{c}\frac{1}{N_{\mu}} \frac{d N}{d P_{T}^{2}} \\
{\left[\left((\mathrm{GeV} / \mathrm{c})^{-2}\right]\right.}\end{array}$ & $\begin{array}{l}\text { stat. Fehler } \\
{\left[(\mathrm{GeV} / \mathrm{c})^{-2}\right]}\end{array}$ \\
\hline 0.0 & $\begin{array}{ll}, \quad 0.3 \\
\end{array}$ & 0.120 & 3.188 & $\pm 9.04510^{-2}$ \\
\hline 0.3 & 0.6 & 0.422 & 0.891 & $\pm 4.49510^{-2}$ \\
\hline 0.6 & 0.9 & 0.727 & 0.368 & $\pm 2.76610^{-2}$ \\
\hline 0.9 & 1.2 & 1.031 & 0.173 & $\pm 1.8611^{-2}$ \\
\hline 1.2 & 1.5 & 1.334 & $7.88110^{-2}$ & $\pm 1.15710^{-2}$ \\
\hline 1.5 & 2.1 & 1.773 & $5.25510^{-2}$ & $\pm 8.02510^{-3}$ \\
\hline 2.1 & 2.7 & 2.364 & $2.20010^{-2}$ & $\pm 4.93010^{-3}$ \\
\hline 2.7 & 3.6 & 3.088 & $1.19110^{-2}$ & $\pm 2.69810^{-3}$ \\
\hline 3.6 & 4.5 & 3.949 & $3.43910^{-3}$ & $\pm 1.03510^{-3}$ \\
\hline 4.5 & 6.0 & 5.098 & $1.26710^{-3}$ & $\pm 5.11610^{-4}$ \\
\hline 6.0 & 7.5 & 6.645 & $8.89910^{-4}$ & $\pm 4.59910^{-4}$ \\
\hline 7.5 & , 10.5 & 8.882 & $2.11010^{-4}$ & $\pm 1.33310^{-4}$ \\
\hline 10.5 & , 15.9 & 12.539 & $1.34210^{-4}$ & $\pm 8.20710^{-5}$ \\
\hline
\end{tabular}

Tabelle A8: $\mathrm{P}_{T}^{2}$-Verteilung für geladene Hadronen 


\begin{tabular}{|c|c|c|c|c|}
\hline $\begin{array}{r}\left.P_{T}^{2}-\right] \\
{[(G}\end{array}$ & $\begin{array}{l}\text { [ntervall } \\
\left.\mathrm{e} V / \mathrm{c})^{2}\right]\end{array}$ & $\begin{array}{c}\left\langle\mathrm{P}_{T}^{2}\right\rangle \\
{\left[(G e V / c)^{2}\right]}\end{array}$ & $\begin{array}{c}\frac{1}{N_{\mu}} \frac{d N}{d P_{T}^{2}} \\
{\left[(G e V / c)^{-2}\right]}\end{array}$ & $\begin{array}{l}\text { stat. Fehler } \\
{\left[(\mathrm{GeV} / \mathrm{c})^{-2}\right]}\end{array}$ \\
\hline \multicolumn{5}{|c|}{$100 \leq \mathrm{W}^{2} \leq 400\left[\mathrm{GeV}^{2}\right]$} \\
\hline 0.0 & 0.3 & 0.119 & 3.633 & \pm 0.143 \\
\hline 0.3 & 0.9 & 0.507 & 0.661 & $\pm 3.99110^{-2}$ \\
\hline 0.9 & 1.8 & 1.213 & 0.101 & $\pm 1.23710^{-2}$ \\
\hline 1.8 & 3.0 & 2.234 & $2.97710^{-2}$ & $\pm 7.62610^{-3}$ \\
\hline 3.0 & 4.5 & 3.559 & $6.23310^{-3}$ & $\pm 2.21410^{-3}$ \\
\hline 4.5 & 6.6 & 4.934 & $8.44610^{-4}$ & $\pm 5.80910^{-4}$ \\
\hline 6.6 & , 14.1 & 8.245 & $1.10310^{-4}$ & $\pm 9.70410^{-5}$ \\
\hline \multicolumn{5}{|c|}{$400 \leq W^{2} \leq 550\left[\mathrm{GeV}^{2}\right]$} \\
\hline 0.0 & 0.3 & 0.120 & 3.032 & \pm 0.153 \\
\hline 0.3 & 0.9 & 0.513 & 0.641 & $\pm 4.73710^{-2}$ \\
\hline 0.9 & 1.8 & 1.219 & 0.116 & $\pm 1.58810^{-2}$ \\
\hline 1.8 & 3.0 & 2.257 & $2.46710^{-2}$ & $\pm 6.15410^{-3}$ \\
\hline 3.0 & 4.5 & 3.808 & $5.92110^{-3}$ & $\pm 2.31810^{-3}$ \\
\hline 4.5 & 6.6 & 5.741 & $1.68310^{-3}$ & $\pm 9.39910^{-4}$ \\
\hline 6.6 & 15.0 & 9.004 & $3.74510^{-4}$ & $\pm 2.61310^{-4}$ \\
\hline \multicolumn{5}{|c|}{$550 \leq W^{2} \leq 1300\left[\mathrm{GeV}^{2}\right]$} \\
\hline 0.0 & $\overline{0.3}$ & 0.122 & 2.265 & \pm 0.109 \\
\hline 0.3 & 0.9 & 0.520 & 0.506 & $\pm 3.46410^{-2}$ \\
\hline 0.9 & 1.8 & 1.230 & $8.61210^{-2}$ & $\pm 1.05910^{-2}$ \\
\hline 1.8 & 3.0 & 2.361 & $2.03810^{-2}$ & $\pm 4.47610^{-3}$ \\
\hline 3.0 & 4.5 & 3.521 & $4.84410^{-3}$ & $\pm 1.61510^{-3}$ \\
\hline 4.5 & 6.6 & 5.883 & $2.33610^{-3}$ & $\pm 1.26710^{-3}$ \\
\hline 6.6 & , 15.9 & 12.267 & $3.25010^{-4}$ & $\pm 1.68910^{-4}$ \\
\hline
\end{tabular}

Tabelle A9: $\mathrm{P}_{T}^{2}$-Verteilung für geladene Hadronen in drei $\mathrm{W}^{2}$-Intervallen 


\begin{tabular}{|c|c|c|c|c|}
\hline \multicolumn{2}{|c|}{$\begin{array}{c}\text { W'}^{2} \text {-Intervall } \\
{\left[\mathrm{GeV}^{2}\right]}\end{array}$} & $\begin{array}{l}<W^{2}> \\
{\left[G e V^{2}\right]}\end{array}$ & $\begin{array}{c}\left\langle\mathrm{P}_{T}^{2}\right\rangle \\
{\left[(\mathrm{GeV} / \mathrm{c})^{2}\right]}\end{array}$ & $\begin{array}{c}\text { stat. Fehler } \\
{\left[(\mathrm{GeV} / \mathrm{c})^{2}\right]}\end{array}$ \\
\hline \multicolumn{5}{|c|}{$0.1 \leq z_{h} \leq 0.2$} \\
\hline 100 & 200 & 188 & 0.273 & $\pm 5.36810^{-2}$ \\
\hline 200 & 300 & 250 & 0.246 & $\pm 2.46310^{-2}$ \\
\hline 300 & 400 & 348 & 0.276 & $\pm 2.98610^{-2}$ \\
\hline 400 & 500 & 449 & 0.312 & $\pm 3.56310^{-2}$ \\
\hline 500 & 700 & 586 & 0.292 & $\pm 2.84610^{-2}$ \\
\hline 700 & , 1000 & 774 & 0.358 & $\pm 5.22410^{-2}$ \\
\hline \multicolumn{5}{|c|}{$0.2 \leq \mathrm{z}_{h} \leq 0.4$} \\
\hline 100 & 200 & 187 & 0.300 & $\pm 6.81510^{-2}$ \\
\hline 200 & 300 & 247 & 0.380 & $\pm 4.80010^{-2}$ \\
\hline 300 & 400 & 348 & 0.397 & $\pm 5.77710^{-2}$ \\
\hline 400 & 500 & 448 & 0.444 & $\pm 7.03910^{-2}$ \\
\hline 500 & 700 & 588 & 0.561 & $\pm 8.53410^{-2}$ \\
\hline 700 &, 1000 & 769 & 0.502 & \pm 0.100 \\
\hline \multicolumn{5}{|c|}{$0.4 \leq z_{h} \leq 1.0$} \\
\hline 100 & 300 & 236 & 0.544 & \pm 0.106 \\
\hline 300 & 500 & 390 & 0.740 & \pm 0.115 \\
\hline 500 & 700 & 592 & 0.724 & \pm 0.170 \\
\hline
\end{tabular}

Tabelle A10: Mittleres $\mathrm{P}_{T}^{2}$ gegen $\mathrm{W}^{2}$ in drei $z_{h}$-Intervallen

\begin{tabular}{|c|c|c|c|c|}
\hline \multicolumn{2}{|c|}{$z_{h}^{2}$-Intervall } & \multirow{2}{*}{$\begin{array}{c}\left\langle z_{h}^{2}\right\rangle \\
1.54110^{-2}\end{array}$} & \multirow{2}{*}{$\begin{array}{c}\begin{array}{c}\left\langle P_{T}^{2}>\right. \\
{\left[(\mathrm{GeV} / \mathrm{c})^{2}\right]}\end{array} \\
0.262\end{array}$} & \multirow{2}{*}{$\begin{array}{c}\text { stat. Fehler } \\
{\left[(\mathrm{GeV} / \mathrm{c})^{2}\right]} \\
\pm 1.65710^{-2}\end{array}$} \\
\hline 0.0100 &,$\quad 0.0225$ & & & \\
\hline 0.0225 &,$\quad 0.0400$ & $3.02610^{-2}$ & 0.313 & $\pm 2.49310^{-2}$ \\
\hline 0.0400 & , 0.0900 & $6.03310^{-2}$ & 0.404 & $\pm 3.23210^{-2}$ \\
\hline 0.0900 &,$\quad 0.1600$ & 0.120 & 0.491 & $\pm 5.80410^{-2}$ \\
\hline 0.1600 &,$\quad 0.2500$ & 0.200 & 0.539 & $\pm 9.82310^{-2}$ \\
\hline 0.2500 &,$\quad 0.3600$ & 0.295 & 0.687 & \pm 0.136 \\
\hline 0.3600 & , 0.6400 & 0.464 & 0.708 & \pm 0.161 \\
\hline 0.6400 & , 1.0000 & 0.749 & 0.508 & \pm 0.221 \\
\hline
\end{tabular}

Tabelle A11: Mittleres $\mathrm{P}_{T}^{2}$ gegen $\mathrm{z}_{h}^{2}$ 


\section{Verzeichnis der Abbildungen}

2.1 Ein-Photon-Austausch Feynman-Graph . . . . . . . . . . 3

$2.2 \nu W_{2}$ gegen $\omega\left(\omega=1 / x_{B j}\right)$ für festen Elektronenstreuwinkel $\left(\theta=10^{\circ}\right)$ und $E_{0}$ im Bereich von 7-17.7 GeV gemessen am SLAC [7] . . . . 6

$2.3 \mu$-Nukleon-Streuprozeß im Parton-Modell . . . . . . . . . . 6

2.4 Quarkdichteverteilungen im Proton nach [12], a) $Q^{2}=1.8(\mathrm{GeV} / \mathrm{c})^{2}$,

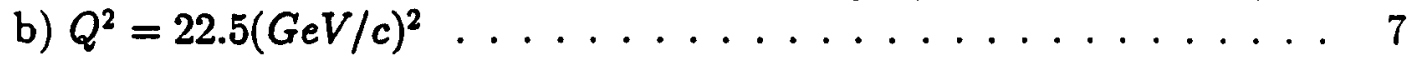

$2.5 \quad F_{2}^{\mu p}\left(\right.$ EMC) gegen $Q^{2}$ für verschiedene $\mathrm{x}$-Bins (aus [13] ) $\ldots \ldots 8$

2.6 QCD-Prozesse in erster Ordnung $\alpha_{a}:$ a) Gluon-Bremsstrahlung, b) $q \bar{q}$ Paarbildung und c) Gluon-Selbstkopplung $\ldots \ldots \ldots \ldots$

2.7 QCD-Korrekturen, die exemplarisch zur a) Skalenbrechung und b) Faktorisierungsbrechung führen $\ldots \ldots \ldots \ldots 11$

2.8 Fragmentierungsprozeß im Field-Feynman-Modell $[24] \ldots \ldots \ldots$

2.9 String-Fragmentierung im Lund-Modell $[27] \ldots \ldots \ldots$

2.10 Partonschauerentwicklung am Beispiel der $\mathrm{e}^{+} \mathrm{e}^{-}$-Annihilation [23]. . 15

2.11 Hadronisierung im Kluster-Fragmentierungs-Modell [23]. . . . . . 15

3.1 Gesamtüberblick über die a) Fermilab-Beschleuniger und b) Strahlführungen $[32] \ldots \ldots \ldots \ldots \ldots \ldots \ldots \ldots \ldots$

3.2 Schematischer Aufbau der NM-Strahlführung . . . . . . . 20

3.3 Das Strahlprofil am Ort der Strahlmeßstation 4. a) horizontales und vertikales Strahlprofil, b) Impulsverteilung für die 500-GeV-Justierung (nach Anbringen der Auswahlkriterien aus Tabelle [5.1]) . . . . . . 21

3.4 Der Detektor des Experimentes 665 am Fermilab . . . . . . . . . 23

3.5 $1 / \beta$ gegen den Impuls für Elektronen, Pionen, Kaonen, Protonen und Deuteronen zusammen mit Vorhersagen (durchgezogene Kurven) nach

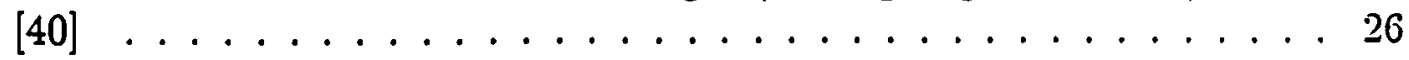


3.6 Tiefinelastisches Streuereignis in Auf- und Seitenansicht des Vertexbe-

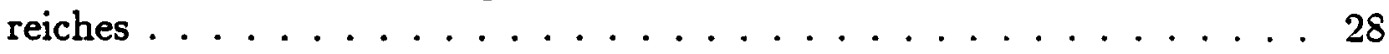

3.7 Erwartete gegen gemessene Anzahl von Photoelektronen für a) Simulation und b) Daten im Čerenkovzähler C0 [43] . . . . . . . . . . 29

3.8 Der schematische Aufbau des RICH-Detektors . . . . . . . . . 31

3.9 Ringabbildung des RICH-Detektors für ein Beamtriggersignal . . . . 31

3.10 Identifikationsbereiche der einzelnen Detektoren . . . . . . . . . 32

3.11 Der geometrische Aufbau des elektromagnetischen Kalorimeters . . . 33

3.12 Datenfluß der Datenerfassung . . . . . . . . . . . . . 36

4.1 Datenfluß der E665-Rekonstruktions-Software-Kette für elektronische Daten des Vorwärtsspektrometers (ohne Teilchenidentifikation). . 41

$4.2 \chi^{2}$-Wahrscheinlichkeitsverteilung für myonische Spuren im Vorwärtsspektrometer . . . . . . . . . . . . . . 45

4.3 Programmfluß innerhalb des Particle Identification Program . . . . 47

4.4 Rechnerstruktur und -leistung des Fermilab-Rechenzentrums ${ }^{6} \ldots .49$

5.1 a) $\mathrm{Q}^{2}$ - und b) $\mathrm{Y}_{B_{j}}$-Verteilung sowie die normierten, differentiellen $\mathrm{c}$ ) $z$ - und d) $\mathrm{P}_{T}^{2}$-Verteilungen der 500-GeV-Deuterium-Daten (nach der Filterstufe, ohne Korrekturen im Vergleich zu Monte-Carlo-Vorhersagen) 51

5.2 $\mathrm{Q}^{2}$ vs. $\mathrm{Y}_{B j}$ a) und b) generierte Größen c), d) rekonstruierte Größen für inkorrekt rekonstruierte Myonen. (a),c): $0<Q^{2}<200 \mathrm{GeV}^{2} / \mathrm{c}^{2}, \quad 0<$ $\left.\left.\left.Y_{B j}<1, \mathrm{~b}\right), \mathrm{d}\right): 0<Q^{2}<10 \mathrm{GeV}^{2} / c^{2}, 0.6<Y_{B j}<1\right) \ldots \ldots \ldots$

$5.3 W^{2}-$ Akzeptanz a) vor und b) nach der Ereignisauswahl . . . . . 55

5.4 Position der primären Vertices in Strahlrichtung im Intervall a) $-17 \mathrm{~m}$ $--4 m$ und $b)-12 m--10 m \ldots \ldots \ldots \ldots \ldots$

$5.5 \mathrm{P}_{T}^{2}$-Auflösung für hadronische Spuren $\ldots \ldots \ldots \ldots \ldots$ 
5.6 Verhältnis der 'ungenau' rekonstruierten hadronischen Spuren zu allen hadronischen Spuren in Abhängigkeit vom Raumabstand zum primären Vertex. . . . . . . . . . . . . . . 66

5.7 Abstand der hadronischen Spuren vom primären Vertex für a) Daten und b) Simulation. . . . . . . . . . . . . . . . 61

5.8 Akzeptanz-Korrekturfunktionen für a) z und b) $\mathrm{P}_{T}^{2} \ldots \ldots \ldots 4$

5.9 Ein-Photon-Austausch Feynman-Diagramm + QED-Prozesse höherer Ordnungen . ....................... 65

5.10 Strahlungskorrekturfaktor sowie kohärente und inelastische Beiträge zum Wirkungsquerschnitt für $x_{B j}=0.5$ und 0.03 gegen $y_{B j} \ldots \ldots 67$

5.11 Strahlungskorrekturfunktion für a) $z_{h}$ und b) $P_{T}^{2} \ldots \ldots 67$

5.12 a) Rohe und b) vollständig korrigierte $Y_{B_{j}}$-Verteilung im Vergleich zum Lund-Modell. . . . . . . . . . . . . . . . . . . . . . 68

5.13 Akzeptanz- und strahlungskorrigierte normierte z-Verteilung im $\mathrm{W}^{2}-$ Intervall $300 \mathrm{GeV}^{2}<W^{2}<400 \mathrm{GeV}^{2}$ für zwei unterschiedliche Sätze von Auswahlkriterien. . . . . . . . . . . . . 70

$6.1 \mathrm{z}$-Spektrum für positive und negative Hadronen $\ldots \ldots \ldots \ldots$

6.2 Ladungsverhältnis in Abhängigkeit von $z$ im Vergleich zum LUNDModell (Version 4.3) . . . . . . . . . . . . 7

$6.3 \mathrm{z}$-Verteilung für geladene Hadronen gemessen in diesem Experiment im Vergleich zu Messungen der EMC und CHIO . . . . . . . . 76

$6.4 \mathrm{z}$-Verteilung für geladene Hadronen gemessen in diesem Experiment im Vergleich zur $\nu \mathrm{N}-$ und $\bar{\nu} \mathrm{N}-$ Streuung. . . . . . . . . 78

6.5 Feynman-Graph zur CC-Reaktion $\ldots \ldots \ldots \ldots \ldots$

$6.6 z$-Verteilung für geladene Hadronen gemessen in diesem Experiment im Vergleich zur $\mathrm{e}^{+} \mathrm{e}^{-}-$Vernichtung . . . . . . . . . . . 81 
$6.7 z$-Verteilung für geladene Hadronen gemessen in diesem Experiment im Vergleich zur $\mathrm{e}^{+} \mathrm{e}^{-}-$Vernichtung bei annähernd gleicher invarianter Masse $\mathrm{W} \sim 20 \mathrm{GeV} \ldots \ldots \ldots . \ldots . \ldots . \ldots 83$

6.8 Z-Verteilungen für a) drei $x_{B j}-$, und jeweils vier b) $\nu-$, c) $Q^{2}-$ und d) $\mathrm{W}^{2}$-Intervalle

6.9 Steigungsparameter $b$ der Anpassung $e^{a+b z}$ an die z-Verteilungen gegen a) $x_{B j}$, b) $\nu$, c) $Q^{2}$ und d) $W^{2} \ldots \ldots \ldots \ldots 6$

6.10 Steigungsparameter $\mathrm{b}$ der Anpassung $\mathrm{e}^{a+b z}$ an die z-Verteilungen gegen $W^{2}$ für dieses Experiment, Daten der EMC und $e^{+} e^{-}-$AnnihilationsExperimente $(z>0.1) \ldots \ldots \ldots \ldots$. . . . . . . 87

6.11 Normiertes differentielles $\mathrm{P}_{T}^{2}$-Spektrum der E665-Daten im Vergleich zu Messungen $\operatorname{der}$ EMC . . . . . . . . . . . . . . ss

6.12 Normiertes differentielles $\mathrm{P}_{T}^{2}$-Spektrum der E665-Daten im Vergleich zu Messungen aus der $\nu$-Nukleon-Streuung . . . . . . . . . 89

6.13 $\mathrm{P}_{T}^{2}$-Spektrum der E665-Daten in drei $\mathrm{W}^{2}$-Intervallen im Vergleich zu Messungen der EMC . . . . . . . . . . . . . 91

6.14 Mittleres $\mathrm{P}_{T}^{2}$ gegen $\mathrm{W}^{2}$ für drei verschiedene z-Intervalle $\ldots \ldots \ldots 92$

6.15 Mittleres $\mathrm{P}_{T}^{2}$ gegen $\mathrm{W}^{2}$ für a) $0.1<z<0.2$ b) $0.2<z<0.4$ c) $0.4<$ $z<1.0 \mathrm{im}$ Vergleich zu Daten der EMC und Neutrino-Experimenten

6.16 Mittleres $P_{T}^{2}$ gegen $z^{2}$ im Vergleich zu Daten a) der EMC und b) Neutrino-Experimenten . . . . . . . . . . . . 95

6.17 Mittleres $\mathrm{P}_{T}^{2}$ gegen $\mathrm{W}^{2}$ im Vergleich zu verschiedenen Versionen des Lund-Modells. . . . . . . . . . . . . . . . . 96

6.18 Mittleres $\mathrm{P}_{T}^{2}$ gegen $\mathrm{z}^{2}$ im Vergleich $\mathrm{zu}$ verschiedenen Versionen des Lund-Modells. 


\section{Tabellenverzeichnis}

1.1 Einteilung der fundamentalen Elementarteilchen nach [2] . . . . . 1

2.1 Definition der kinematischen Variablen in der Myon-Nukleon-Streuung 4

3.1 Tevatron Parameter $\ldots \ldots \ldots \ldots \ldots \ldots$

3.2 Mitgliederliste der E665-Kollaboranten $\ldots \ldots \ldots \ldots$

3.3 Parameter des $500-\mathrm{GeV}-\mathrm{Myonstrahles} \ldots \ldots \ldots \ldots \ldots \ldots$

3.4 Parameter der Spurnachweiselemente . . . . . . . . . . . 25

3.5 Datenstatistik der Meßperiode $1987 / 88 \ldots \ldots \ldots \ldots$

5.1 Parameter der Ereignisselektion . . . . . . . . . . . . 57

5.2 Statistik zur Reduktion des Datensatzes . . . . . . . . . . 57

$5.3 \quad$ Parameter $\operatorname{der}$ Spurselektion . . . . . . . . . . . . . . 62

5.4 Mittlere systematische Fehler in $z_{h}, \mathrm{P}_{T}^{2}$ und $\left\langle P_{T}^{2}>\ldots \ldots . \ldots 74\right.$

6.1 Quark-Strahl-Zusammensetzung in der tiefinelastischen Leptoproduktion und $\mathrm{e}^{+} \mathrm{e}^{-}$-Annihilation $\ldots \ldots \ldots \ldots \ldots . \ldots . \ldots 79$

A1 $z$-Verteilung für positive Hadronen $\ldots \ldots \ldots \ldots$

A2 $z$-Verteilung für negative Hadronen $\ldots \ldots \ldots \ldots \ldots$

A3 $\quad z$-Verteilung für geladene Hadronen . . . . . . . . . . . . . 102

A4 $z$-Verteilung für geladene Hadronen in drei verschiedenen $x_{B j}$-Intervallen 103

A5 $\mathrm{z}$-Verteilung für geladene Hadronen in vier verschiedenen $\nu$-Intervallen 104

A6 $z$-Verteilung für geladene Hadronen in vier verschiedenen $Q^{2}$-Intervallen1 105

A7 z-Verteilung für geladene Hadronen in vier verschiedenen $W^{2}$-Intervallen 106

A8 $\quad \mathrm{P}_{T}^{2}$-Verteilung für geladene Hadronen $\ldots \ldots \ldots \ldots \ldots$ 
A9 $\quad \mathrm{P}_{T}^{2}$-Verteilung für geladene Hadronen in drei $\mathrm{W}^{2}$-Intervallen $\ldots \ldots 108$ A10 Mittleres $\mathrm{P}_{T}^{2}$ gegen $\mathrm{W}^{2}$ in drei $z_{h}$-Intervallen $\ldots \ldots \ldots . \ldots 109$ A11 Mittleres $\mathbf{P}_{T}^{2}$ gegen $z_{h}^{2} \ldots \ldots \ldots \ldots \ldots$ 


\section{Literaturverzeichnis}

[1] E.Rutherford, The Scattering of $\alpha$ and $\beta$ Particles by Matter and the Structure of the Atom,Philosophical Magazine, Series IV,21(1911) 669

[2] Otto Nachtmann, Phänomene und Konzepte der Elementarteilchenphysik, Friedr. Vieweg \& Sohn, Braunschweig/Wiesbaden, 1986

[3] Fermilab E665 proposal, Muon Scattering with Hadron Detection at the Tevatron, 1980

[4] F.E.Close, An Introduction to Quarks and Partons, Academic Press, London/New York/San Francisco, 1979

[5] S.D.Drell, J.D.Walecka, Electrodynamic Processes with Nuclear Targets, Ann. Phys. 28 (1964) 18

[6] J.D.Bjorken, Asymptotic Sum Rules at Infinite Momentum, Phys. Rev. 179 (1969) 1547

[7] E.D.Bloom et al., High-Energy Inelastic e-p Scattering at $6^{\circ}$ and $10^{\circ}$, Phys. Rev. Lett. 23 (1969) 930

M.Breidenbach et al., Observed Behavior of Highly Inelastic Electron-Proton Scattering, Phys. Rev. Lett. 23 (1969) 935

G.Miller et al., Inelastic Electron-Proton Scattering at Large Momentum Transfers and the Inelastic Structure Functions of the Proton, Phys. Rev. D 5 (1972) 528

[8] C.G.Callan, D.J. Gross, High-Energy Electroproduction and the Constitution of the Electric Current, Phys. Rev. Lett. 22 (1969) 156

[9] J.D.Bjorken, E.A.Paschos, Inelastic Electron-Proton and $\gamma$-Proton Scattering and the Structure of the Nucleon, Phys. Rev. 185 (1969) 1975

R.P.Feynman, Photon-Hadron Interactions, W.A.Benjamin, Inc., Advanced Book Program, Reading, Massachusetts (1972)

[10] J.Drees, Deep Inelastic Scattering, Lectures given at the 1980 CERN School of Physics, Malente, June 8-21, 1980 ; WU B 80-34

[11] M.Gell-Mann, A Schematic Model of Baryons and Mesons, Phys. Lett. 8 (1964) 214

[12] A.J.Buras, Asymptotic Freedom in Deep Inelastic Processes in the Leading Order and Beyond, Rev. Mod. Phys. 52 (1980) 199 
[13] J.Drees, H.E.Montgomery, Muon Scattering, Ann. Rev. Nucl. Part. Sci. 33 (1983) 383

[14] Particle Data Group, Review of Particle Properties, Phys. Lett. 170B (1986) p.73

[15] J.Kogut, L.Susskind, Scale-invariant Parton Model, Phys. Rev. D 9 (1974) 697 J.Kogut, L.Susskind, Parton Models and Asymptotic Freedom, Phys. Rev. D 9 (1974) 3391

[16] G.Altarelli, G.Parisi, Asymptotic Freedom in Parton Language, Nucl. Phys. B126 (1977) 298

[17] N.Schmitz, Recent Results on the Hadronic Final State in Charged Current Neutrino and Antineutrino Reactions, Proceedings of the 1979 International Symposium on Lepton and Photon Interactions at High Energies, Fermilab, August 23-29, 1979, p.359

[18] T.Sloan et al., The Quark Structure of the Nucleon from the CERN Muon Experiments, Phys. Rep. 162 (1988) 45.

[19] R.Baier, K.Fey, Finite Corrections to Quark Fragmentation Functions in Perturbative QCD, Z. Physik C, Particles and Fields 2 (1979) 339

[20] J.J.Aubert et al., A Search for Free Quarks in Deep Inelastic Muon Scattering, Phys. Lett. 133B (1983) 461

[21] L.W.Jones, A Review of Quark Search Experiments, Rev. Mod. Phys. 49 (1977) 717

[22] G.S.LaRue et al., Evidence for the Existence of Fractional Charge on Matter, Phys. Rev. Lett. 38 (1977) 1011

[23] T.Sjöstrand, Status of Fragmentation Models, Int. Jour. Mod. Phys. A3 (1988) 751

[24] R.D.Field, R.P.Feynman, A Parametrization of the Properties of Quark Jets, Nucl. Phys. B136 (1978) 1

[25] B.Andersson et al., Parton Fragmentation and String Dynamics, Phys. Rep. 97 (1983) 31

[26] T.Sjöstrand, The Lund Monte Carlo for Jet Fragmentation, Comp. Phys. Comm. 27 (1982) 243 
[27] M.Pötsch, Untersuchungen der Hadronproduktion in Endzuständen der tiefinelastischen Myon-Nukleon-Streuung, Dissertation, Universität-GHSWuppertal, WU B-DI 86-5, November 1986

[28] W.Bartel et al., Experimental Study of Jets in Electron-Positron Annihilation, Phys. Lett. 101B (1981) 129;

U.Bartel et al., Test of Fragmentation Models by Comparison with Three-Jet Events Produced in $e^{+} e^{-} \rightarrow$ Hadrons, Phys. Lett. 134B (1984) 275

[29] J.R.Sanford, The Fermi National Accelerator Laboratory, Ann. Rev. Nucl. Sci. 26 (1976) 151

[30] R.Rubinstein, Fermilab Research Program, Workbook (1989)

[31] L.M.Lederman, Fermilab 1978-1989, Annual Report of the Fermi National Accelerator Laboratory (1988)

[32] H. T. Edwards, The Tevatron Energy Doubler: A Superconducting Accelerator, Ann. Rev. Nucl. Part. Sci. 35 (1985) 605

[33] R. Palmer, A.V. Tollestrup, Superconducting Magnet Technology for Accelerators, Ann. Rev. Nucl. Part. Sci. 34 (1984) 247

[34] J.Peoples, The Fermilab Antiproton Source, IEEE Trans. Nucl. Sci. NS-30 (1983) 1970

[35] A.Malensek,J.G.Morfin, The Tevatron Muon Beam: A High Intensity Beam with Well Defined Polarization, FNAL TM-1193 (1983)

[36] J.P. Albanese et al., The Vertex and Large Angle Detectors of a Spectrometer System for High Energy Muon Physics, Nucl. Inst. and Meth. 212 (1983) 111

[37] W.R.Francis,T.B.W.Kirk, Muon Scattering at Fermilab, Phys. Rep. 54C (1979) 307

[38] M.R.Adams et al., A Spectrometer for Muon Scattering at the Tevatron, Nucl. Inst. and Meth. A291 (1990) 533

[39] U.Ecker, Determination of the Liquid Target Densities in the E665 Run 1987/1988, E665 interner Bericht, AN025 (1988)

[40] M.Erdmann, Lebensdauer des Farbigen Protons in der Myon-Proton-Streuung, Dissertation, Albert-Ludwigs-Universität Freiburg i.Brsg., März 1990

[41] M.Pötsch, Ein Gas-Schwellencerenkovzähler für den Vertexdetektor der EMC am SPS-Muonstrahl am CERN, Diplomarbeit, Universität-GHS-Wuppertal, WU D 81-11, Oktober 1981 
[42] U.Ecker, C0-Alignment (I) : Frame Alignment, E665 Interner Bericht, VS021 (1988)

[43] U.Ecker, C0 Analysis Status, E665 Collaboration Meeting, Harvard, December 8-10, 1988, TR094

[44] S.K.Dhawan et al., A RICH MWPC Pad Readout by Using Custom Microplex I. C., IEEE Trans. Nucl. Sci. 35 (1988) 436

[45] G.B.Coutrakon et al., The Ring Imaging Cherenkov Detector for Fermilab Experiment 665, IEEE Trans. Nucl. Sci. 35 (1988) 470

[46] H.Venkataramania, The E665 RICH Counter, Symposium on Particle Identification at High Luminosity Hadron Colliders, April 5-7, 1989, FNAL

[47] S.Aïd et al., The E665 (TMC) E-M Calorimeter, Proceedings of the Gas Sampling Calorimetry Workshop II, Held at Fermilab, October $31^{\text {st }}$ and November $1^{\text {st }}$, 1985, p. 249

[48] E.J.Ramberg, Neutral Pion and Eta Production in Deep Inelastic Muon Scattering at $480 \mathrm{GeV}$, Dissertation, University of Maryland, 1989

[49] S.Magill, $\mathrm{Xe} / \mathrm{D}_{2}$ Cross Section Ratio from Muon Scattering at $490 \mathrm{GeV} / \mathrm{c}$, Dissertation, University of Illinois at Chicago, 1990

[50] U.Ecker, Scaler Studies First Attempt, E665 interner Bericht, AN015 (1988)

[51] D.F.Geesaman et al., Data Acquisition for FNAL E665, IEEE Trans. Nucl. Sci. 36 (1989) 1528

[52] R.Brun et al., ZEBRA-Kernel Data Management System, CERN Program Library, DD/EE/85-6

[53] J.J.Ryan, E665 Structure Chart, E665 interner Bericht, SW053 (1988)

[54] M.Schmitt, S.Wolbers, Standardization of PTMV, E665 interner Bericht, SW100 (1989)

[55] H.Melanson, E665 Wire Chamber Pattern Recognition Program User's Guide, E665 interner Bericht, SW073 (1988)

D.M.Jansen, A User's Guide to the PC/PCF Pattern Recognition Program, E665 interner Bericht, SW069 (1988)

[56] H.Wind, Momentum Analysis by Osing a Quintic Spline Model for the Track, Nucl. Inst. Meth. 115 (1974) 431

H.Wind, An Improvement to Iterative Tracking for Momentum Determination, Nucl. Inst. Meth. 153 (1978) 195 
[5T] A.Salvarani, Description of Track Fitting, E665 interner Bericht, SW040 (1986)

[58] A.A.Bhatti et al., The E665 Vertex Processor, E665 interner Bericht, SW071 (1989)

[59] H.Albrecht et al., Observation of Antideuteron Production in Electron Positron Annihilation at $10 \mathrm{GeV}$ Center of Mass Energy, Desy 85-034 (1985)

[60] H.Braun, Particle Identification in E665, E665 interner Bericht, TR034 (1986)

[61] U.Ecker, E665 Particle Identification Program, E665 interner Bericht, SW052 (1988)

[62] S.Kunori, S.Aïd, E665 Monte Carlo Program, E665 interner Bericht, MC012 (1986)

[63] R.Brun et al., GEANT 9, CERN Data Handling Division, DD/EE/84-1 (1987)

[64] S.Wolbers, The E665 Second Stage Monte Carlo; Version 4.0, E665 interner Bericht, MC010 (1987)

[65] U.Ecker, Preliminary Event Selection for the Study of Hadronic Final States, E665 interner Bericht, AN053 (1989)

[66] N.Pavel, Hadronproduktion in der tief-inelastischen Myon-Kern-Streuung, Dissertation, Universität-GHS-Wuppertal, WUB 89-24, Mai 1989

[67] E.Lohrmann, Hochenergiephysik, Teubner Studienbücher, Stuttgart 1981, p.91

[68] B.Korzen, Die Ladungsstruktur der Nukleonen in der Myoproduktion, Dissertation, Universität-GHS-Wuppertal, WUB 89-7, März 1989

[69] U. Krüner, Transversalimpulsverteilungen der Hadronen in der Muoproduktion, Diplomarbeit, Universität-GHS-Wuppertal, WU B 89-5, Februar 1989

[70] J.Drees, Radiative Corrections and Hadron Distributions in Deep Inelastic $\mu p$ Scattering, EMC/78/24; University of Wuppertal, WU - B 78 - 16, April 1978 Das Programm GAMRAD wurde mir freundlicherweise von N. Pavel zugänglich gemacht.

[71] L.W.Mo,Y.S.Tsai, Radiative Corrections to Elastic and Inelastic ep and $\mu p$ Scattering Rev. Mod. Phys. 41 (1969) 205;

Y.S.Tsai, Radiative Corrections to Electron Scatterings, SLAC-PUB-848 (1971)

[72] A.A.Akhundov et al., Radiative Corrections to Deep Inelastic Muon Scattering, JINR E2-86-104, Dubna 1986 
[73] C.Lietzke, S.J.Wimpenny, A Comparison of the RadEMC and Bardin-Shumeiko Radiative Correction Programs UCR/DIS - 89 - 06, University of California 1989; U.Ecker, Radiative Corrections 'Stage 1', E665 interner Bericht, PH022 (1989)

[74] J.J.Aubert et al., A Measurement of Wide Angle Bremsstrahlung in a High Energy Muon Scattering Experiment as a Check on the Consistency of Radiative Correction Calculations, Z.Phys. C-Particles and Fields 10 (1981) 101

[75] J.J.Aubert et al., Measurement of Hadronic Azimuthal Distributions in Deep Inelastic Muon Proton Scattering, Phys. Lett. 130B (1983) 118.

[76] J.J.Aubert et al.,Scaled Energy Distributions of Single Hadrons Observed in Muon Proton Scattering, Contribution to the 20th International Conference on High Energy Physics, Madison, Wisconsin, 1980; CERN-EP/80-130

[77] W.A.Loomis et al.,Hadron Production in Muon-Proton and Muon-Deuteron Collisions, Phys. Rev. D19 (1979) 2543

[78] M.Derrick et al.,Hadron-Production Mechanisms in Antineutrino-Proton Charged.Current Interactions, Phys. Rev. D24 (1981) 1071

[79] M.Derrick et al., Properties of the Hadronic System Resulting from $\vec{\nu}_{\mu} p$ Interactions Phys. Rev. D17 (1978) 1

[80] L.M.Sehgal, Hadron Production by Leptons, Proceedings of the 1977 International Symposium on Lepton and Photon Interactions at High Energies, Hamburg, August 25-31, 1977, p.837.

[81] R.D.Field and R.P.Feynman, Quark Elastic Scattering as a Source of HighTransverse-Momentum Mesons, Phys. Rev. D15 (1977) 2590

[82] P.C.Bosetti et al., Badron Production in Charged Current Neutrino and Antineutrino Interactions at High Energy, Nucl. Phys. B149 (1979) 13

[83] M.Althoff et al., Jet Production and Fragmentation in $e^{+} e^{-}$Annihilation at 12-49 GeV, Z. Phys. C - Particles and Fields 22 (1984) 307

[84] P.Aarnio et al., Study of Hadronic Decays of the $Z^{0}$ Boson, Phys. Lett. 240B (1990) 271

[85] M.Arneodo et al., Comparison Between Hadronic Final States Produced in $\mu p$ and $e^{+} e^{-}$Interactions, Z. Phys. C - Particles and Fields 35 (1987) 417

[86] T.Uematsu, $Q^{2}$ Dependence of Quark and Gluon Fragmentation Functions in a Parton Picture Based on QCD, Phys. Lett. 79B (1978) 97 
[87] J.F.Owens, On the $Q^{2}$ Dependence of Parton Fragmentation Functions, Phys. Lett. 76B (1978) 85

[88] J.Blietschau et al., Observation of Scaling Deviations in the Energy Distribution of secondary Hadrons in Inelastic Neutrino-Proton Interactions, Phys. Lett. 87B (1979) 281

[89] J.J.Aubert et al., Measurement of the $Q^{2}, x$, and $W^{2}$ Dependence of Single Hadron Production in Deep Inelastic Muon Scattering, Phys. Lett. 114B (1982) 373

W.Stockhausen, Messung von Faktorisierungsbrechung und Skalenverletzung in hadronischen Endzuständen in tiefinelastischer $\mu-p-S t r e u u n g$, Dissertation, Universität-GHS-Wuppertal, WU B DI 82-2, März 1982

[90] M.Arneodo et al., Hadron Multiplicity Variation with $Q^{2}$ and Scale Breaking of the Hadron Distributions in Deep Inelastic Muon-Proton Scattering Phys. Lett. 165B (1985) 222

[91] D.H.Saxon, Quark and Gluon Fragmentation in High Energy $e^{+} e^{-}$ Annihilation, Rutherford Appleton Laboratory, RAL-86-057, July 1986

[92] M.Arneodo et al., Jet Production and Fragmentation Properties in Deep Inelastic Muon Scattering, Z. Phys. C - Particles and Fields 36 (1987) 527

[93] T.Kitagaki et al., Current and Target Jets Produced in High Energy NeutrinoDeuterium Interactions, Phys. Lett. 97B (1980) 325

[94] G.Altarelli, G.Martinelli, Transverse Momentum of Jets in Electroproduction from Quantum Chromodynamics, Phys. Lett. 76B (1978) 89

[95] E.Reya, Perturbative Quantum Chromodynamics, Phys. Rep. 69 (1981) 195

[96] M.Gronau, Y.Zarmi, How to Measure the Transverse Momentum of Partons Phys. Rev. D18 (1978) 2341;

M.Glück, E.Reya, Transverse Momenta of Partons and Dimuons in QCD, Nucl. Phys. B145 (1978) 24;

A.Méndez et al., QCD Effects in Semi-Inclusive Neutrino Processes, Nucl. Phys. B148 (1979) 499

[97] J.J.Aubert et al., Transverse Momentum of Charged Hadrons Observed in Deep Inelastic Muon Scattering, Phys. Lett. 95B (1980) 306

[98] H.Deden et al., Transverse Momentum of Hadrons Produced in $\nu$ and $\bar{\nu}$ Interactions on an Isoscalar Target in BEBC, Nucl. Phys. B181 (1981) 375 
[99] D.Allasia et al., Transverse Momentum of Charged Hadrons Produced in $\nu$ and $\bar{\nu}$ Deuterium Charged Current Interactions, Saclay, DPhPE 84-12 (1984)

[100] P.Allen et al., Transverse Momentum Distributions of Hadrons in Deep Inelastic Neutrino-Proton Scattering, Nucl. Phys. B188 (1981) 1

[101.] Die verschiedenen Programmversionen des Lund-Modelles wurden mir freundlicherweise von U. Krüner zugänglich gemacht.

[102] M.Ameodo et al., Charge and Transverse Momentum Correlations in Deep Inelastic Muon-Proton Scattering, CERN-EP/86-20, February 5th, 1986 
-

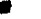

$-$

-

$-$

$=$

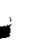

.

-

-

- 